\title{
Synthesis of $N$-protected 1-aminoalkylphosphonium
}

\section{salts from amides, carbamates, lactams, or imides}

\author{
Jakub Adamek, ${ }^{1,2, *}$ Paulina Zieleźny, ${ }^{1}$ and Karol Erfurt ${ }^{3}$
}

\begin{abstract}
${ }^{1}$ Department of Organic Chemistry, Bioorganic Chemistry and Biotechnology, Silesian University of Technology, B. Krzywoustego 4, 44-100 Gliwice, Poland

${ }^{2}$ Biotechnology Centre of Silesian University of Technology, B. Krzywoustego 8, 44-100 Gliwice, Poland

${ }^{3}$ Department of Chemical Organic Technology and Petrochemistry, Silesian University of Technology, B. Krzywoustego 4, 44-100 Gliwice, Poland
\end{abstract}

*Corresponding author: Jakub Adamek, e-mail: jakub.adamek@polsl.pl

\section{Supporting information}

\section{Experimental and analytical data}

Table of contents

1. Apparatus for the one-pot synthesis of $N$-protected 1 -aminoalkylphosphonium salts $\mathbf{1}$

2. A brief comparison of the selected (most important) methods for the synthesis of $N$-protected 1aminoalkylphosphonium salts S3-S4

3. ${ }^{1} \mathrm{H}$ NMR, ${ }^{13} \mathrm{C}\left\{{ }^{1} \mathrm{H}\right\}$ NMR, ${ }^{31} \mathrm{P}$ NMR spectra of compounds 1, 10a-c, 11a, 14 and 15. $. \mathrm{S} 5-\mathrm{S} 122$

4. IR spectra of compounds $\mathbf{1}, \mathbf{1 0 a}-\mathbf{c}, \mathbf{1 1 a}, \mathbf{1 4}$ and $\mathbf{1 5}$ S123-S161

5. MS spectra of all unknown compounds

S162-S190 


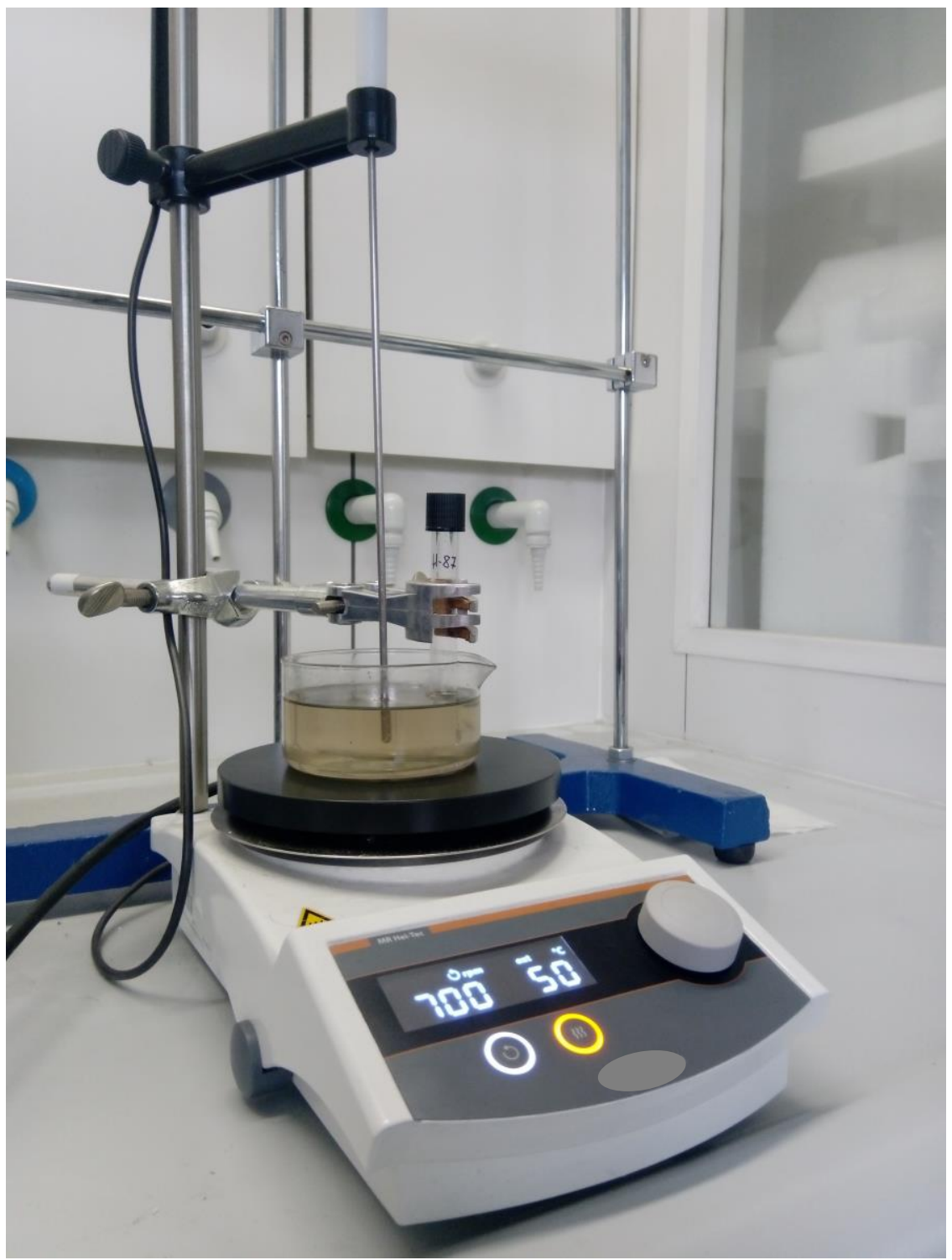

Photo S1. Apparatus for the one-pot synthesis of $N$-protected 1-aminoalkylphosphonium salts 1 (personal photo made by authors). 
Table S1. A brief comparison of the selected (most important) methods for the synthesis of $N$-protected 1-aminoalkylphosphonium salts.

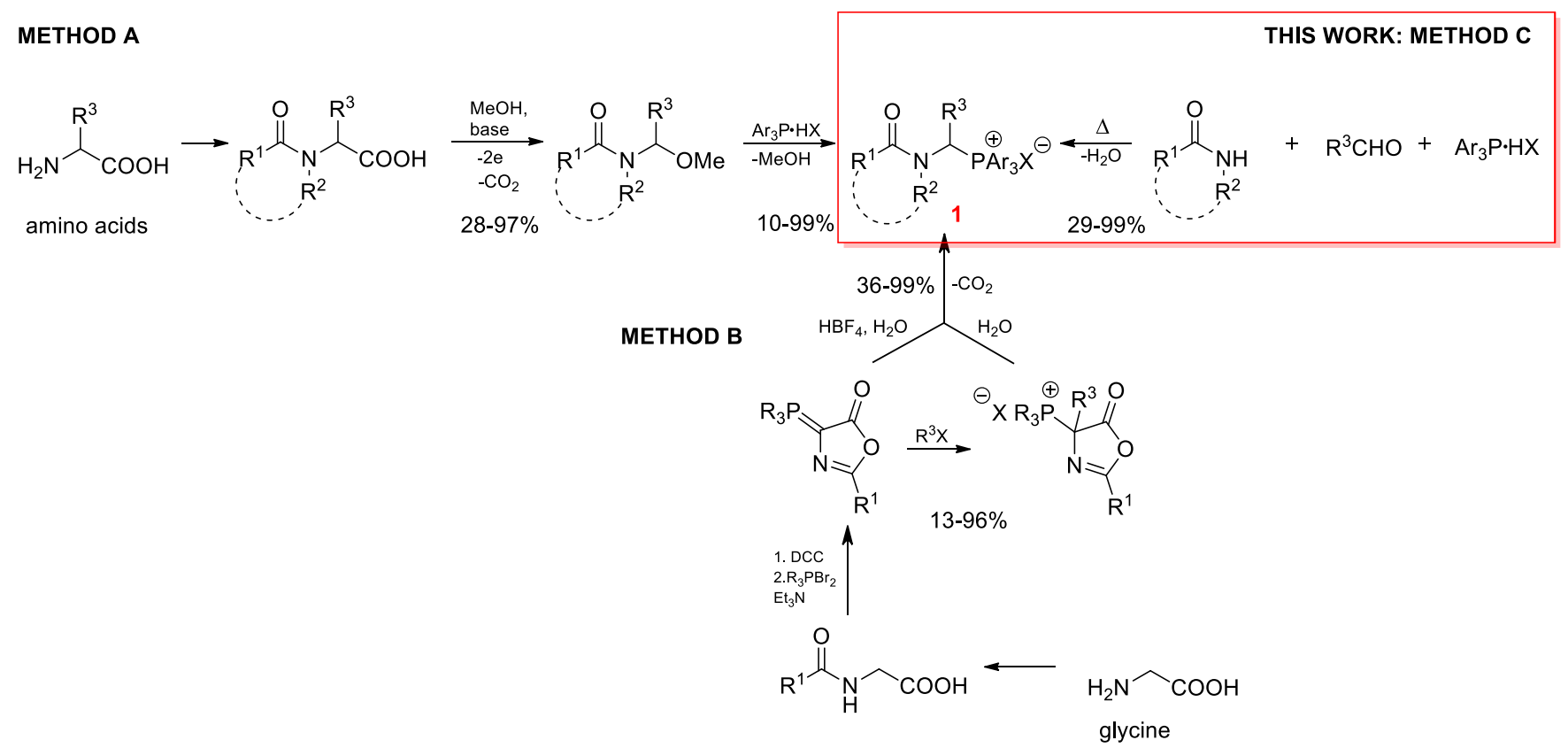

\begin{tabular}{|c|c|c|c|c|}
\hline Method & Substrates & Steps/example & Limitations & Literature \\
\hline A & $\alpha$-amino acids & $\begin{array}{l}\text { 1. } N \text {-protection of } \alpha \text {-amino acids }(\mathbf{1 h}: \\
\text { 2. electrochemical alkoxylation } \\
\text { 3. C-P bond formation } \\
\mathbf{1 g}\left(\mathrm{R}^{1}=\mathrm{Me}, \mathrm{R}^{2}=\mathrm{R}^{3}=\mathrm{H}, \mathrm{Ar}=\mathrm{Ph}, \mathrm{X}=\mathrm{BF}_{4}\right) \text { : } \\
1-2 \text { workdays (reaction and purification time): step } \\
1:^{6} \text { r.t., } 24 \mathrm{~h}, 89-92 \% \text {; step } 2: 10^{\circ} \mathrm{C}, 2 \mathrm{~h}, 96 \% \text {, step } 3 \text { : } \\
60^{\circ} \mathrm{C}, 30 \mathrm{~min}, 98 \% \text {; overall yield: } 84 \%-87 \%\end{array}$ & $\begin{array}{l}\text { electrochemical equipment (electrolyzer, platinum } \\
\text { electrodes, power supply) }\end{array}$ & $1-3$ \\
\hline $\mathrm{B}$ & Glycine, (oxazolones) & $\begin{array}{l}\text { 1. } N \text {-protection of glycine } \\
\text { 2. synthesis of oxazolones } \\
\text { (3.) } 4 \mathrm{C} \text {-alkylation } \\
\text { 4. hydrolysis } \\
\text { 5. decarboxylation } \\
\text { 1g: } 3-4 \text { workdays: step } 1:^{6} \text { r.t., } 24 \mathrm{~h}, 89-92 \% \text {; step } 2 \text { : } \\
\text { r.t., } 24 \mathrm{~h}, 51 \% \text {, step } 3: \text { r.t. } 10 \mathrm{~min}, 97 \% \text {; step } 4 \text { : } \\
105^{\circ} \mathrm{C}, 1 \mathrm{~h}, 99 \% \text {; overall yield: } \mathbf{4 4 - 4 5 \%}\end{array}$ & $\begin{array}{l}\text { multi-step, labor-intensive, time-consuming, narrow } \\
\text { scope of application }\end{array}$ & 4,5 \\
\hline $\mathrm{C}$ & $\begin{array}{l}\text { Aldehydes, amides, } \\
\text { carbamates, imides, } \\
\text { lactams, urea }\end{array}$ & $\begin{array}{l}\text { 1. three-component coupling } \\
\text { 1g: } 135^{\circ} \mathrm{C}, 2-3 \mathrm{~h}, \mathbf{9 1 \%}\end{array}$ & $\begin{array}{l}\text { difficulties in the preparation of } N \text {-protected 1- } \\
\text { aminoalkylphosphonium salts, which are derivatives } \\
\text { of phosphines substituted with electron-withdrawing } \\
\text { substituents (solvent-free methodology) }\end{array}$ & $\begin{array}{l}\text { This } \\
\text { work }\end{array}$ \\
\hline
\end{tabular}


References:

(1) Mazurkiewicz, R.; Adamek, J.; Październiok-Holewa, A.; Zielińska, K.; Simka, W.; Gajos, A.; Szymura, K. $\alpha$-Amidoalkylating Agents from N-Acyl- $\alpha$ amino Acids: 1-(N-Acylamino)alkyltriphenylphosphonium Salts. J. Org. Chem. 2012, 77, 1952-1960, DOI: 10.1021/jo202534u.

(2) Adamek, J.; Węgrzyk-Schlieter, A.; Steć, K..; Walczak, K.; Erfurt, K. Michaelis-Arbuzov-Type Reaction of 1-Imidoalkyltriarylphosphonium Salts with Selected Phosphorus Nucleophiles. Molecules 2019, 24, 3405, DOI: 10.3390/molecules24183405.

(3) Walęcka-Kurczyk, A.; Walczak, K.; Kuźnik, A.; Stecko, S.; Październiok-Holewa, A. The Synthesis of $\alpha$-Aminophosphonates via Enantioselective Organocatalytic Reaction of 1-(N-Acylamino)alkylphosphonium Salts with Dimethyl Phosphite. Molecules, 2020, 25, 405, DOI: 10.3390/molecules25020405.

(4) Mazurkiewicz, R.; Październiok-Holewa, A.; Grymel, M. Synthesis and decarboxylation of $N$-acyl-

$\alpha$-triphenylphosphonio- $\alpha$-amino acids: a new synthesis of $\alpha$-(N-acylamino)alkyltriphenylphosphonium salts. Tetrahedron Lett. 2008, 49, 1801-1803, DOI: 10.1016/j.tetlet.2008.01.051.

(5) Mazurkiewicz, R.; Pierwocha, A.W. Phosphoranylidene-5(4H)-oxazolones - A novel synthesis and properties. Monatsh. Chem. 1996, 127, 219-225, DOI: 10.1007/BF00807402.

(6) Herbst, R.M.; Shemin, D. Acetylglycine. Org. Synth. 1939, 19, 4, DOI: 10.15227/orgsyn.019.0004. 


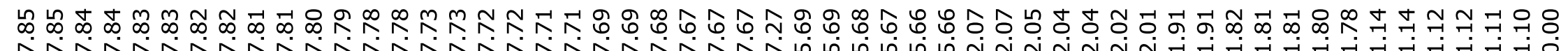

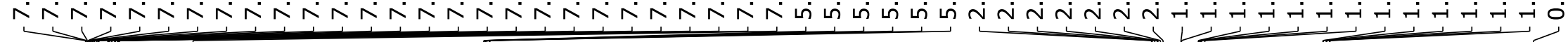

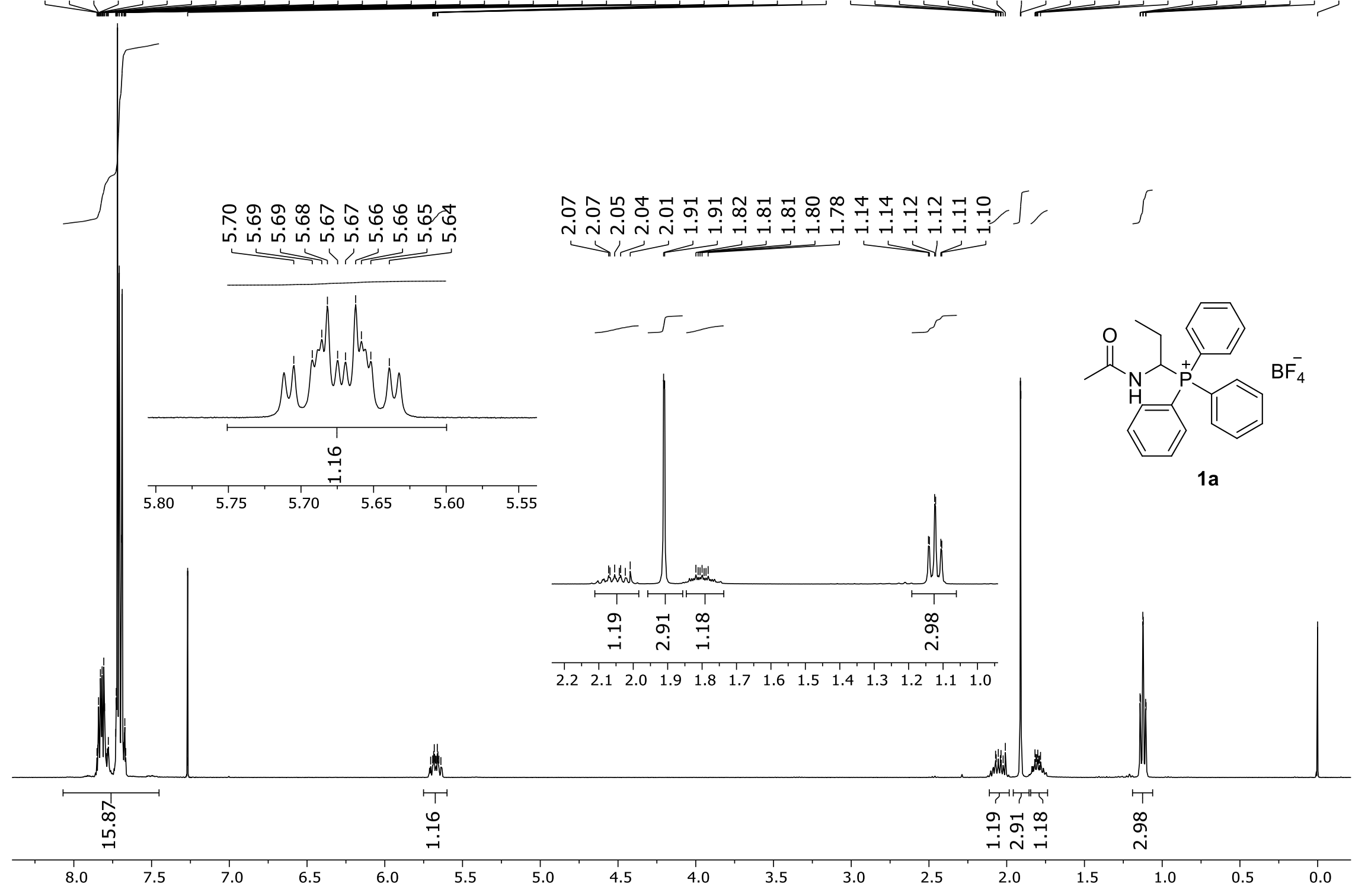

${ }^{1} \mathrm{H}$ NMR spectrum of 1-( $N$-acetylamino)propyltriphenylphosphonium tetrafluoroborate (1a); $400 \mathrm{MHz} / \mathrm{CDCl}_{3} / \mathrm{TMS} ; \delta$ (ppm). 


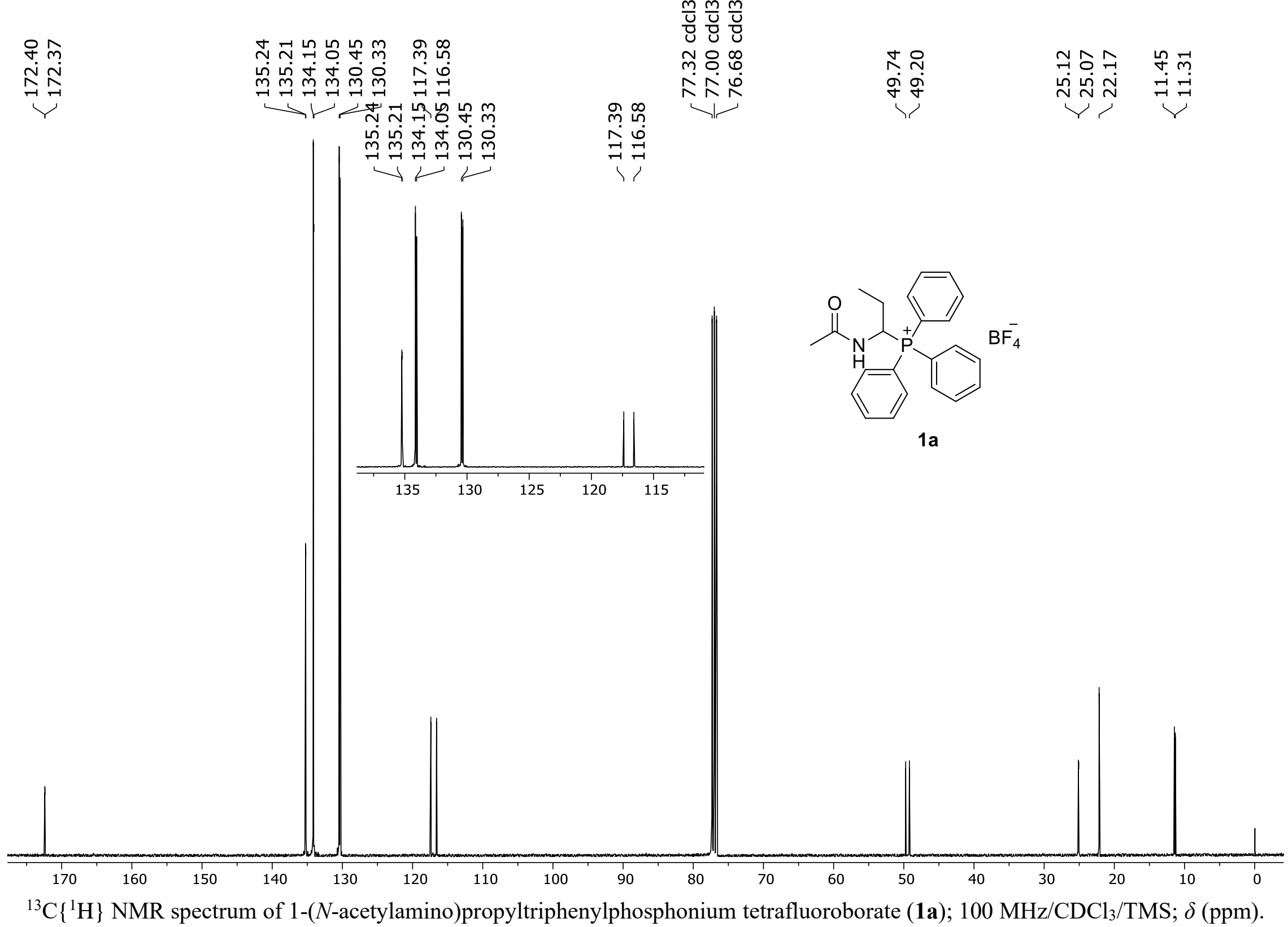




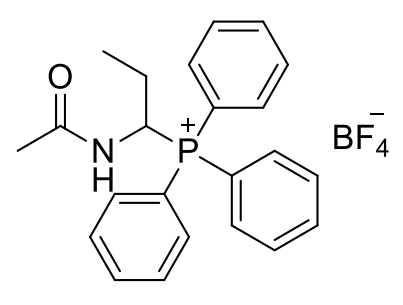

1a

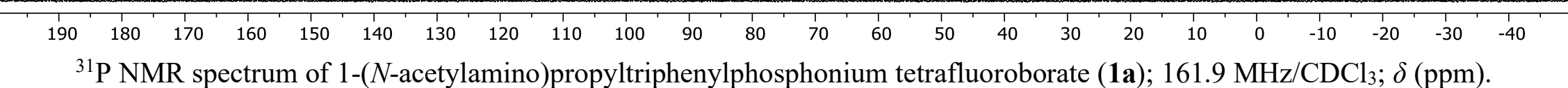




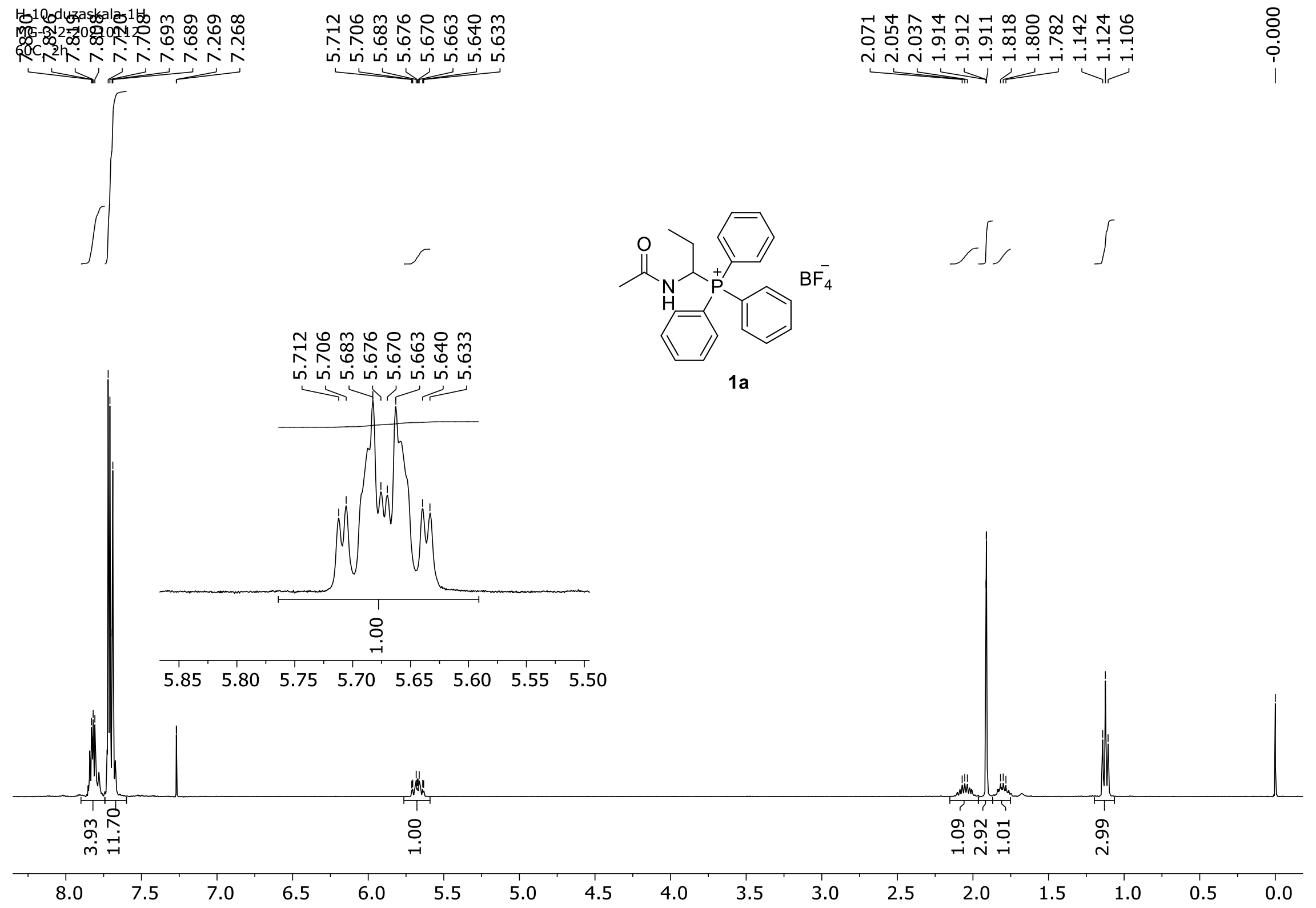

${ }^{1} \mathrm{H}$ NMR spectrum of 1-( $N$-acetylamino)propyltriphenylphosphonium tetrafluoroborate (1a); $400 \mathrm{MHz} / \mathrm{CDCl} / \mathrm{TMS} ; \delta$ (ppm) - synthesis on a $20 \mathrm{~g}$ scale. 


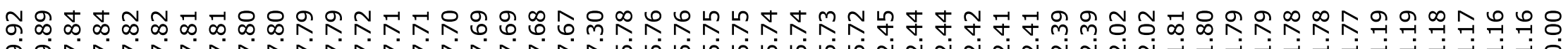
बंள

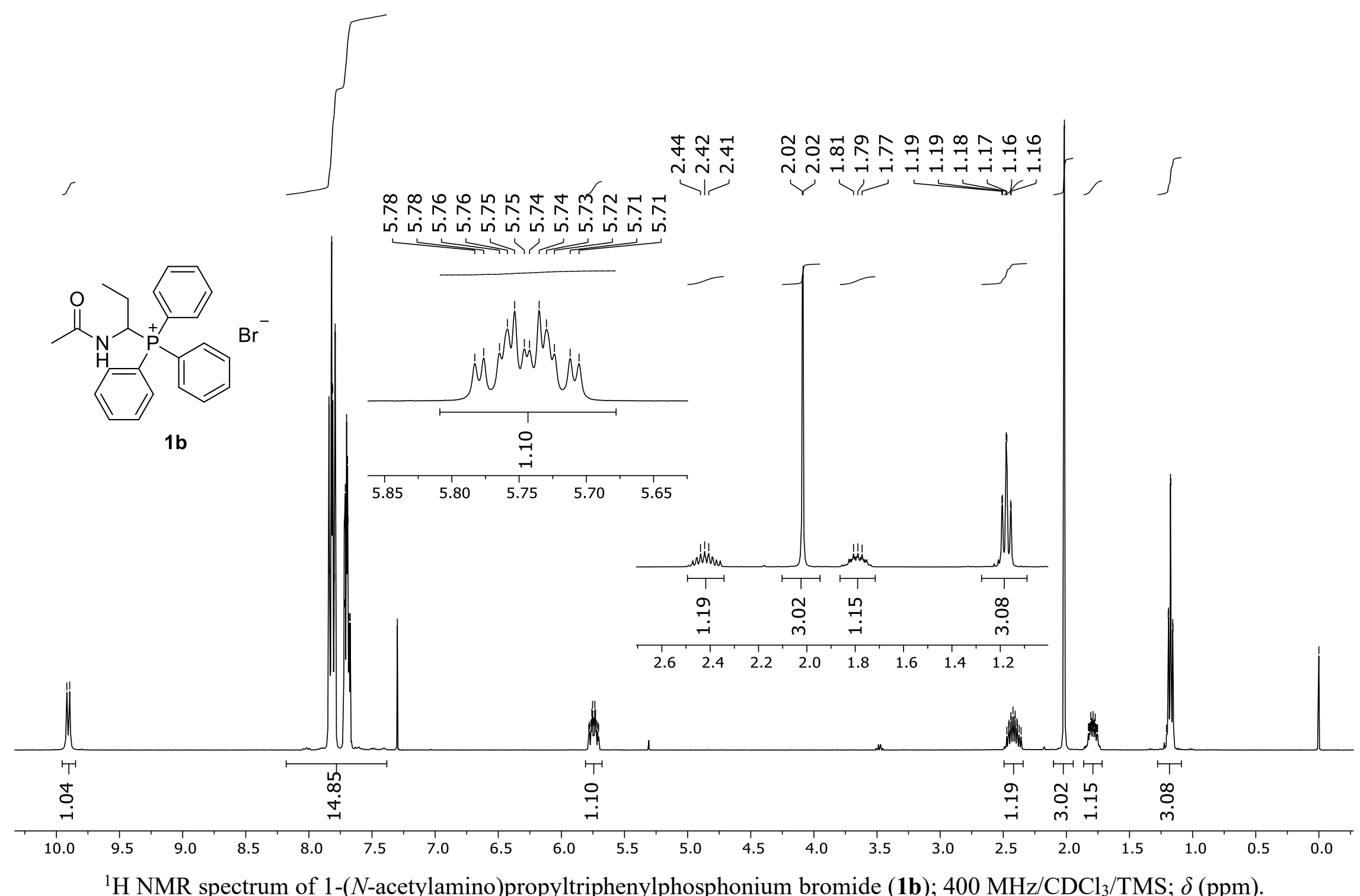

${ }^{1} \mathrm{H}$ NMR spectrum of 1-(N-acetylamino)propyltriphenylphosphonium bromide (1b); $400 \mathrm{MHz} / \mathrm{CDCl}_{3} / \mathrm{TMS} ; \delta$ (ppm). 
ถิก

곤

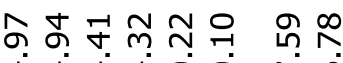

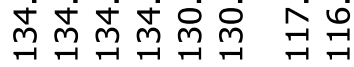

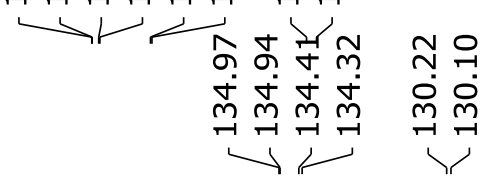

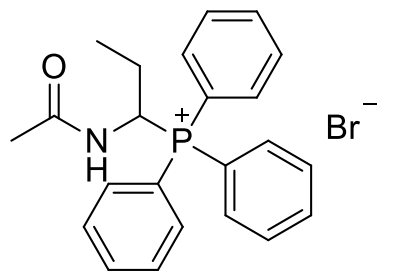

1b

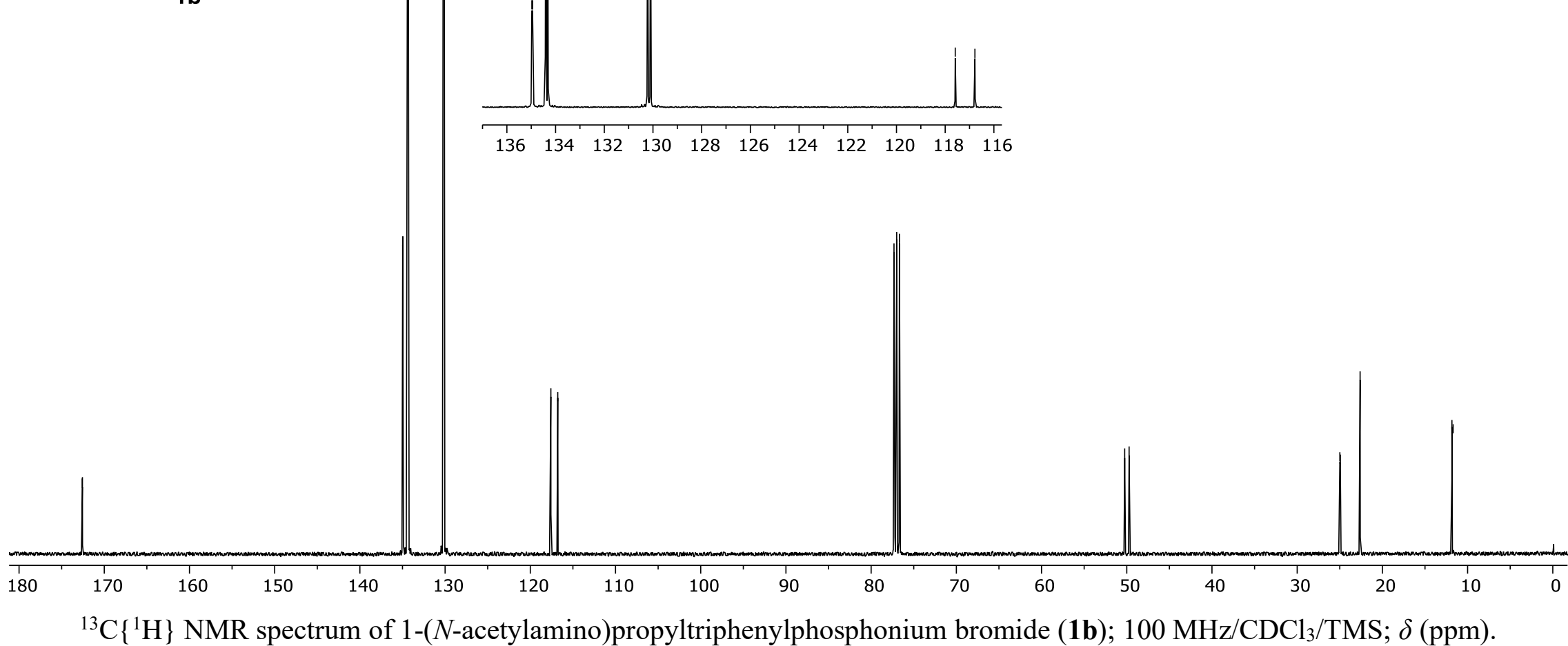




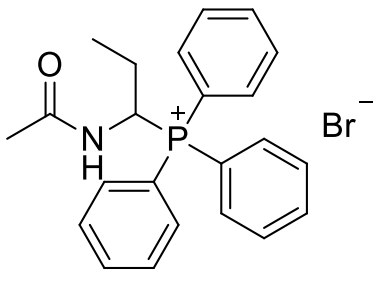

1b

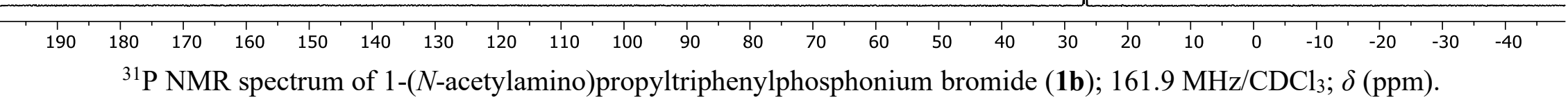



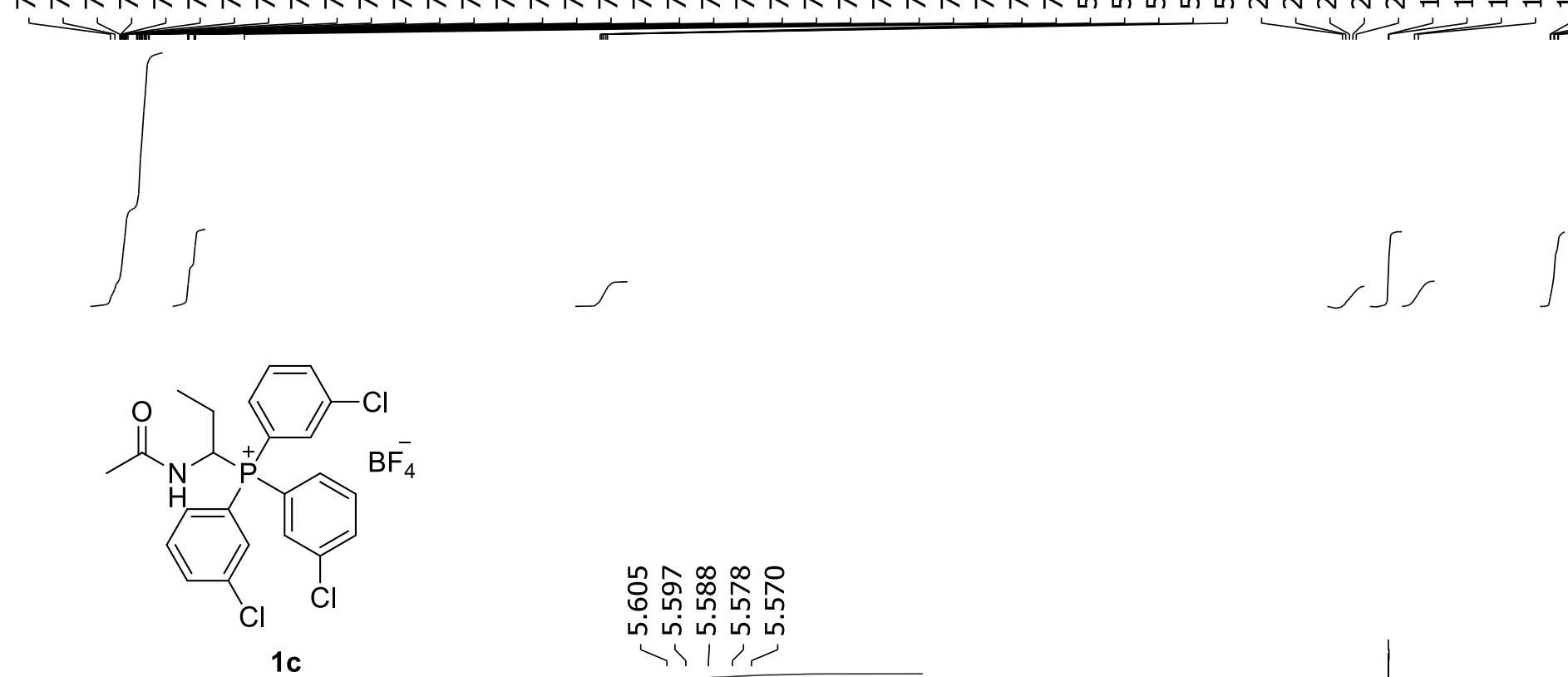

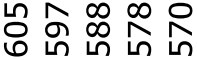

เก เก เก เก

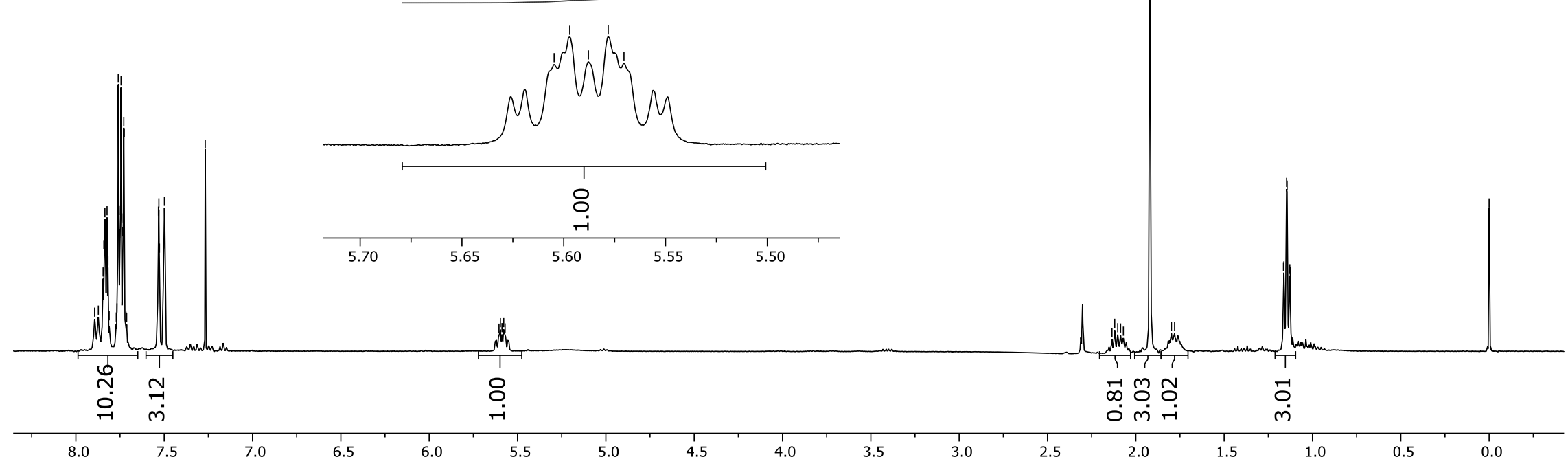

${ }^{1} \mathrm{H}$ NMR spectrum of 1-( $N$-acetylamino)propyltris(3-chlorophenyl)phosphonium tetrafluoroborate (1c); $400 \mathrm{MHz} / \mathrm{CDCl}_{3} / \mathrm{TMS} ; \delta$ (ppm). 
H-40\%్ఞి- छెర్cms

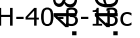

곤

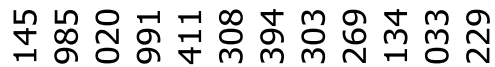

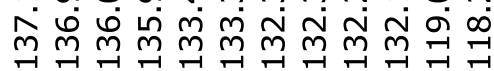

(1)

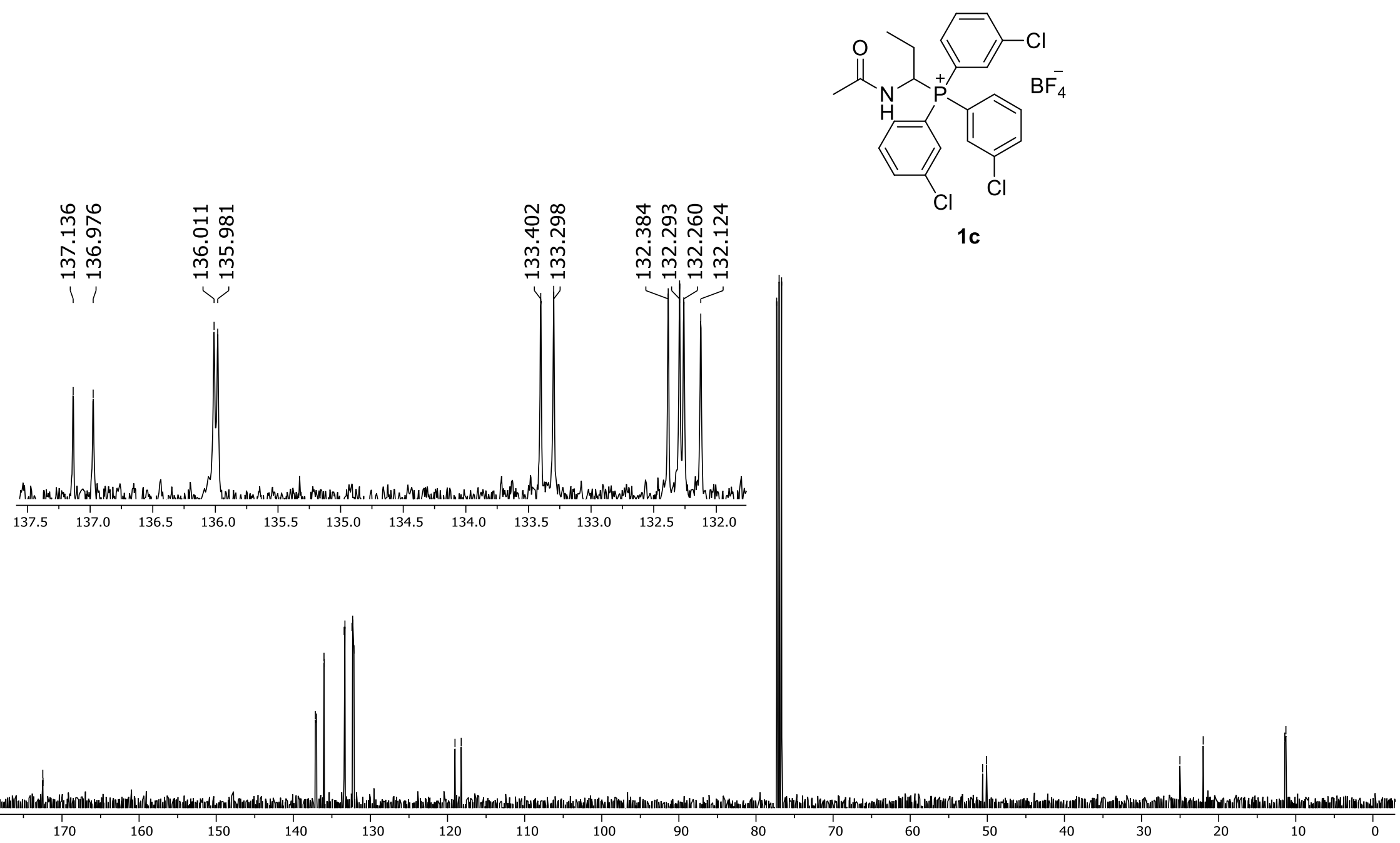

${ }^{3} \mathrm{C}\left\{{ }^{1} \mathrm{H}\right\}$ NMR spectrum of 1-( $N$-acetylamino)propyltris(3-chlorophenyl)phosphonium tetrafluoroborate (1c); $100 \mathrm{MHz} / \mathrm{CDCl}_{3} / \mathrm{TMS} ; \delta$ (ppm). 


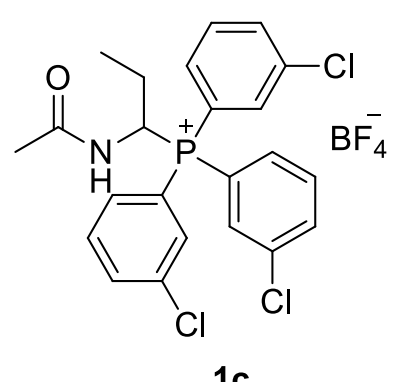

$1 c$

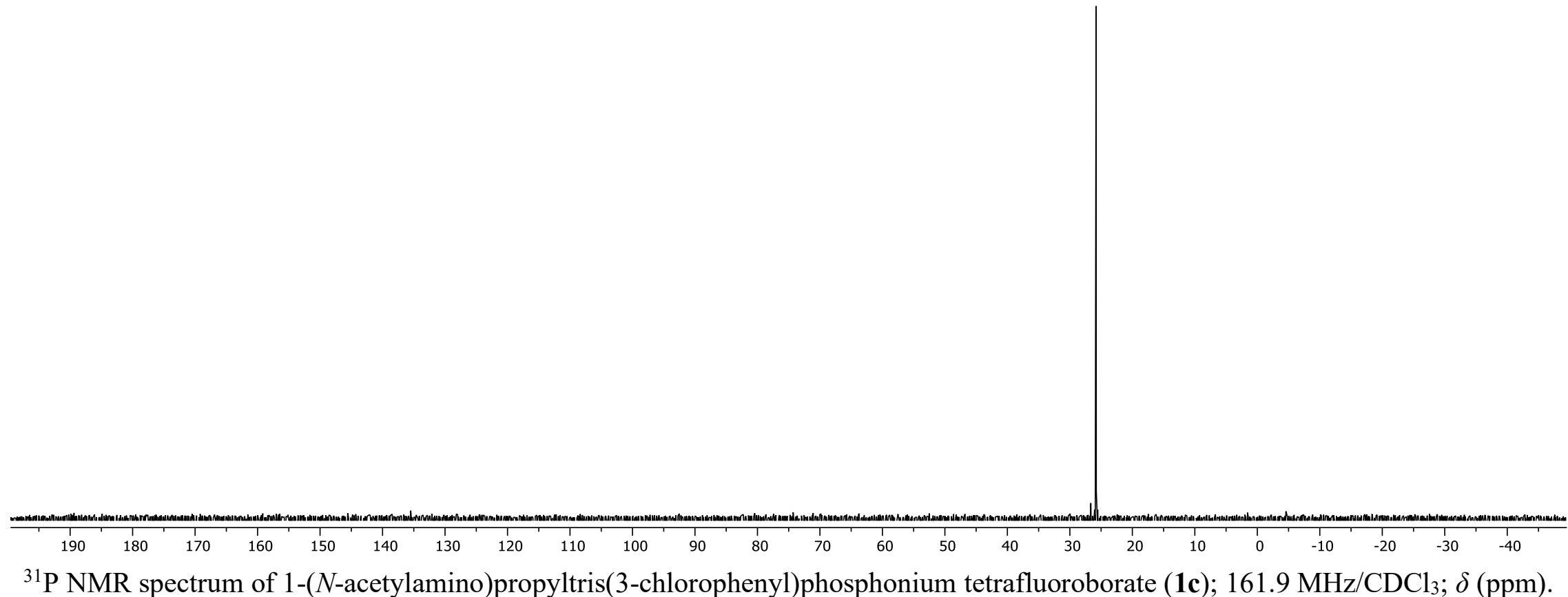




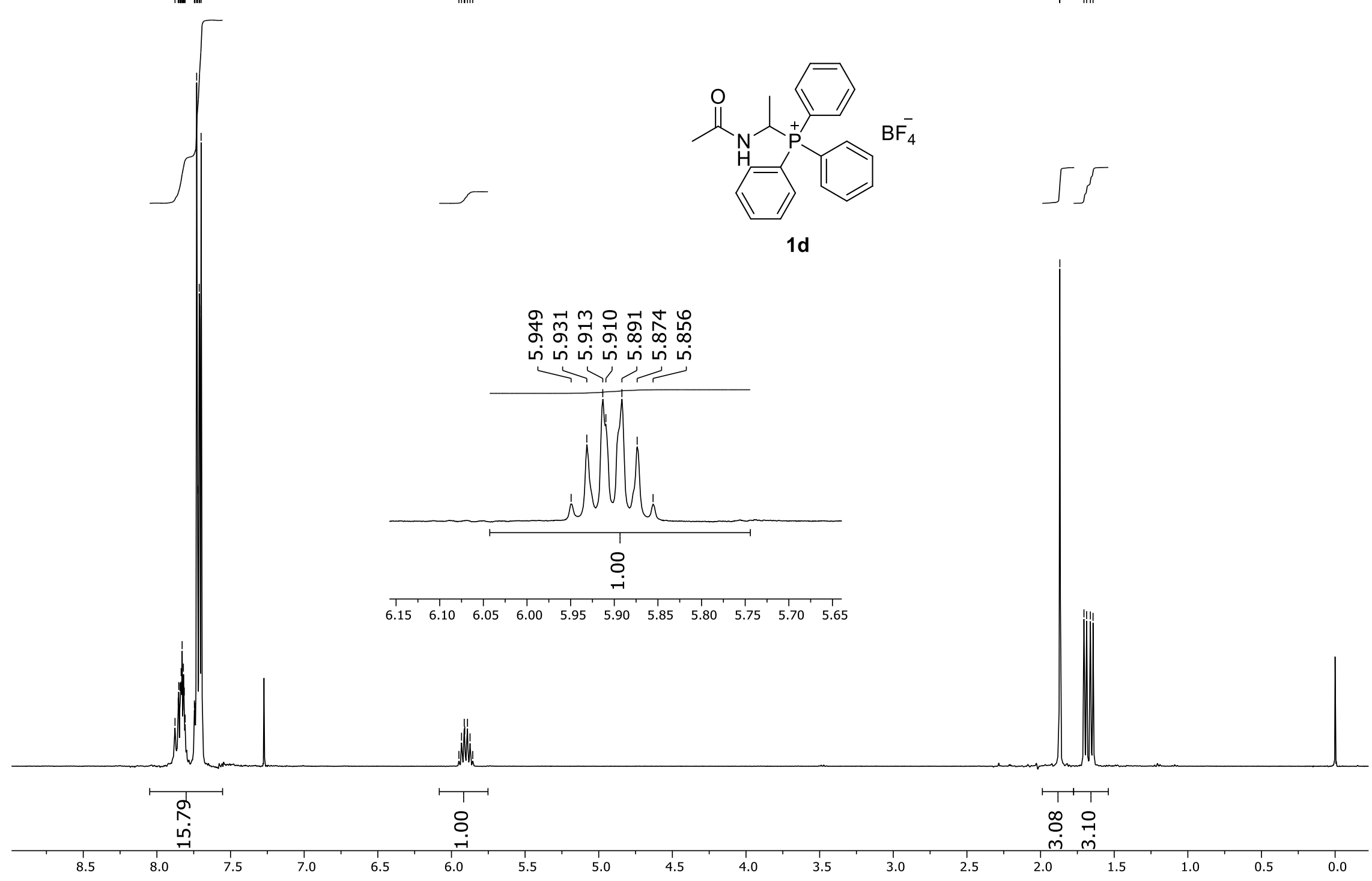

${ }^{1} \mathrm{H}$ NMR spectrum of 1-( $N$-acetylamino)ethyltriphenylphosphonium tetrafluoroborate (1d); $400 \mathrm{MHz} / \mathrm{CDCl} / \mathrm{TMS} ; \delta$ (ppm). 


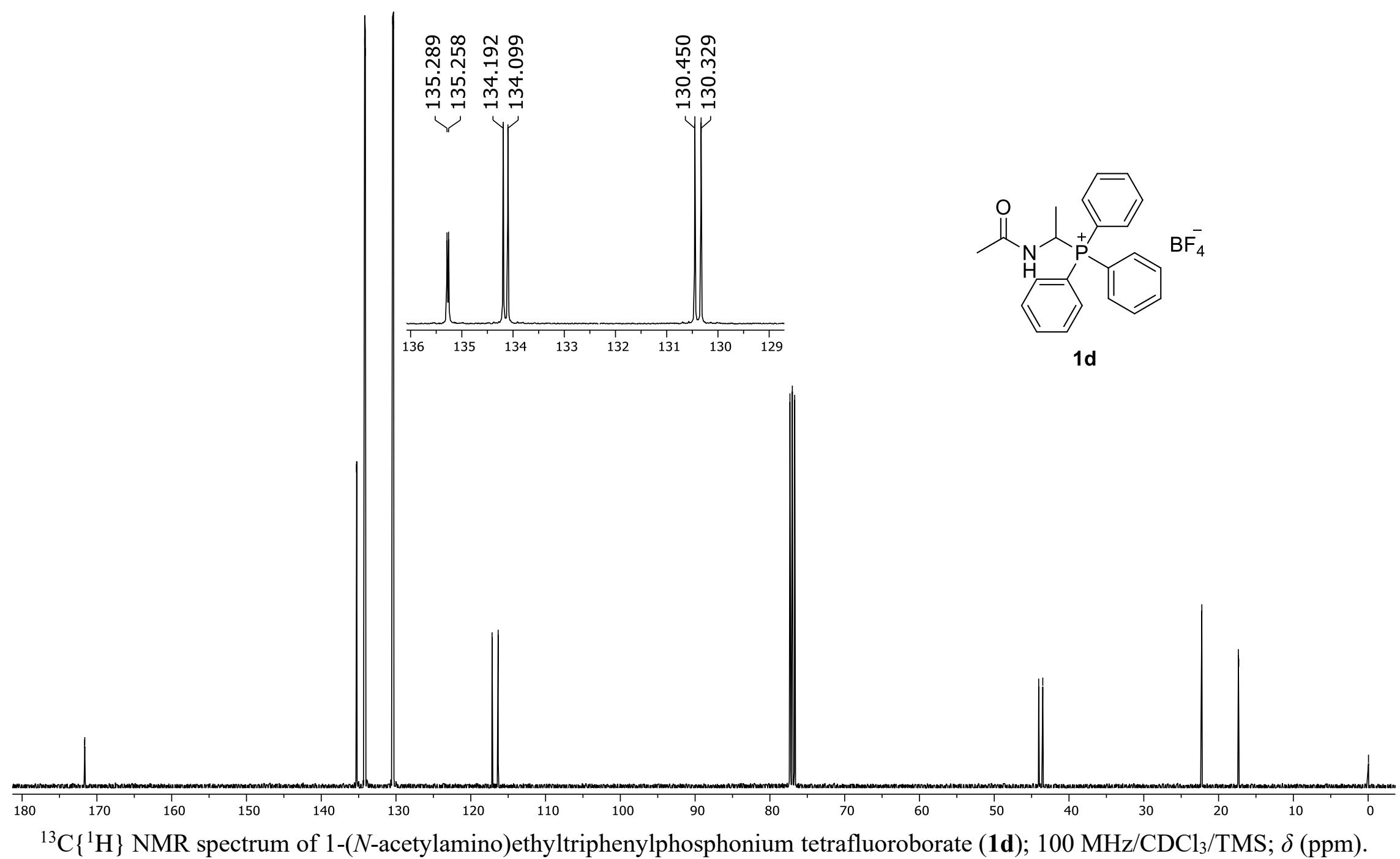




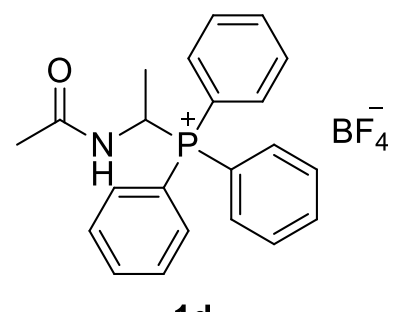

1d

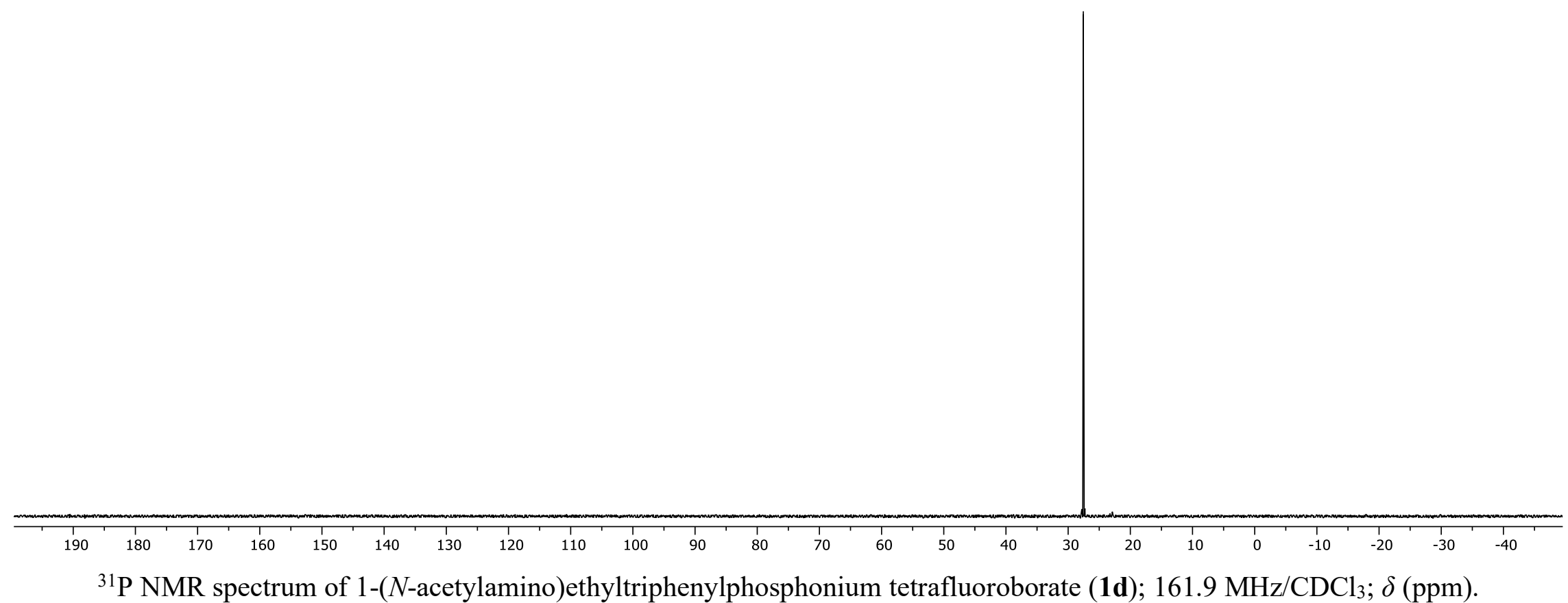




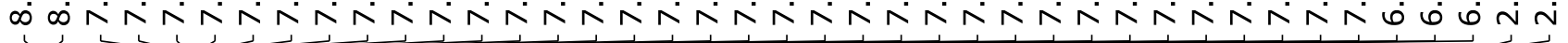

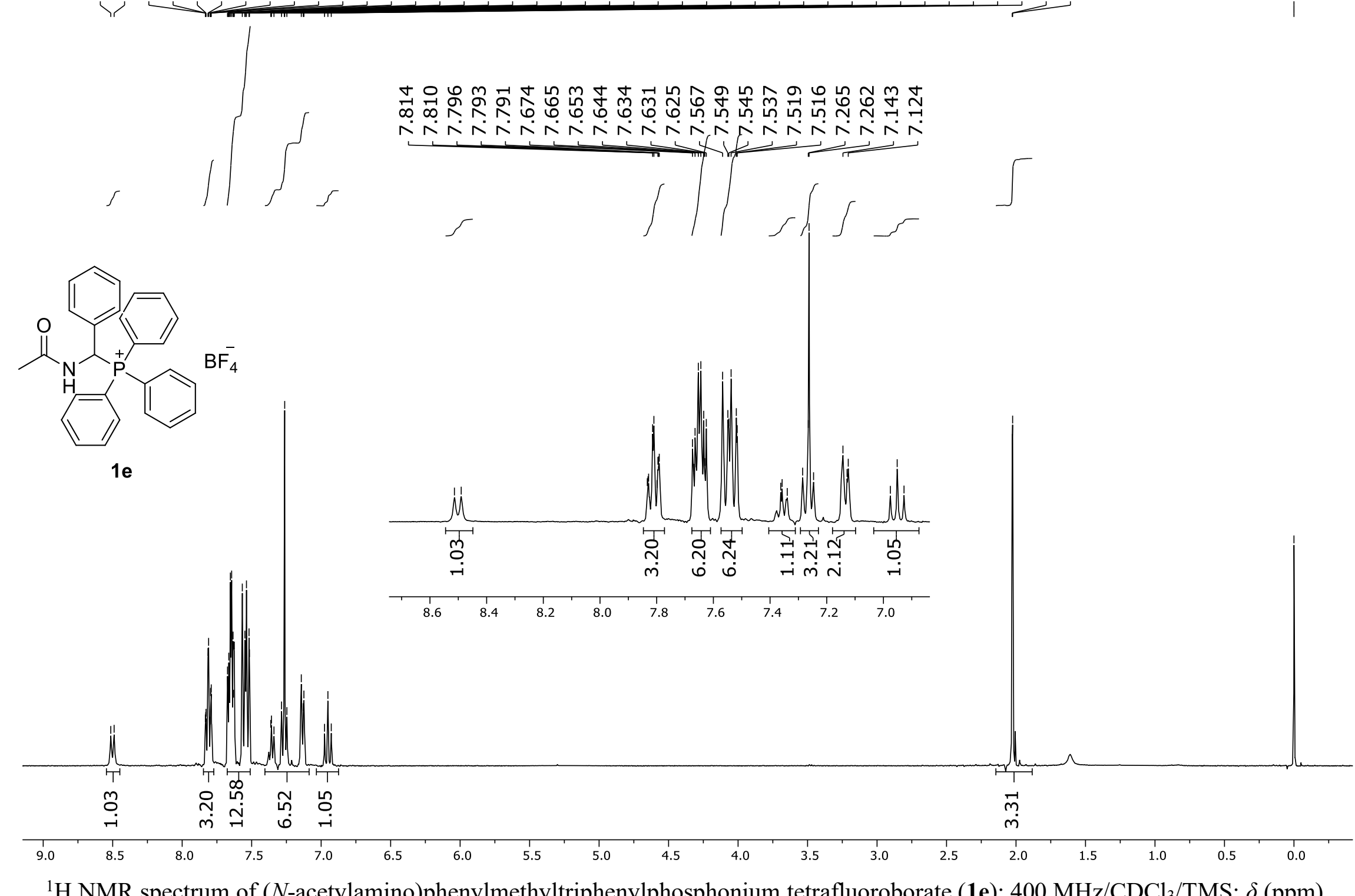

${ }^{1} \mathrm{H}$ NMR spectrum of ( $N$-acetylamino)phenylmethyltriphenylphosphonium tetrafluoroborate (1e); $400 \mathrm{MHz} / \mathrm{CDCl} 3 / \mathrm{TMS} ; \delta$ (ppm). 
苍

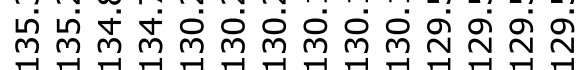

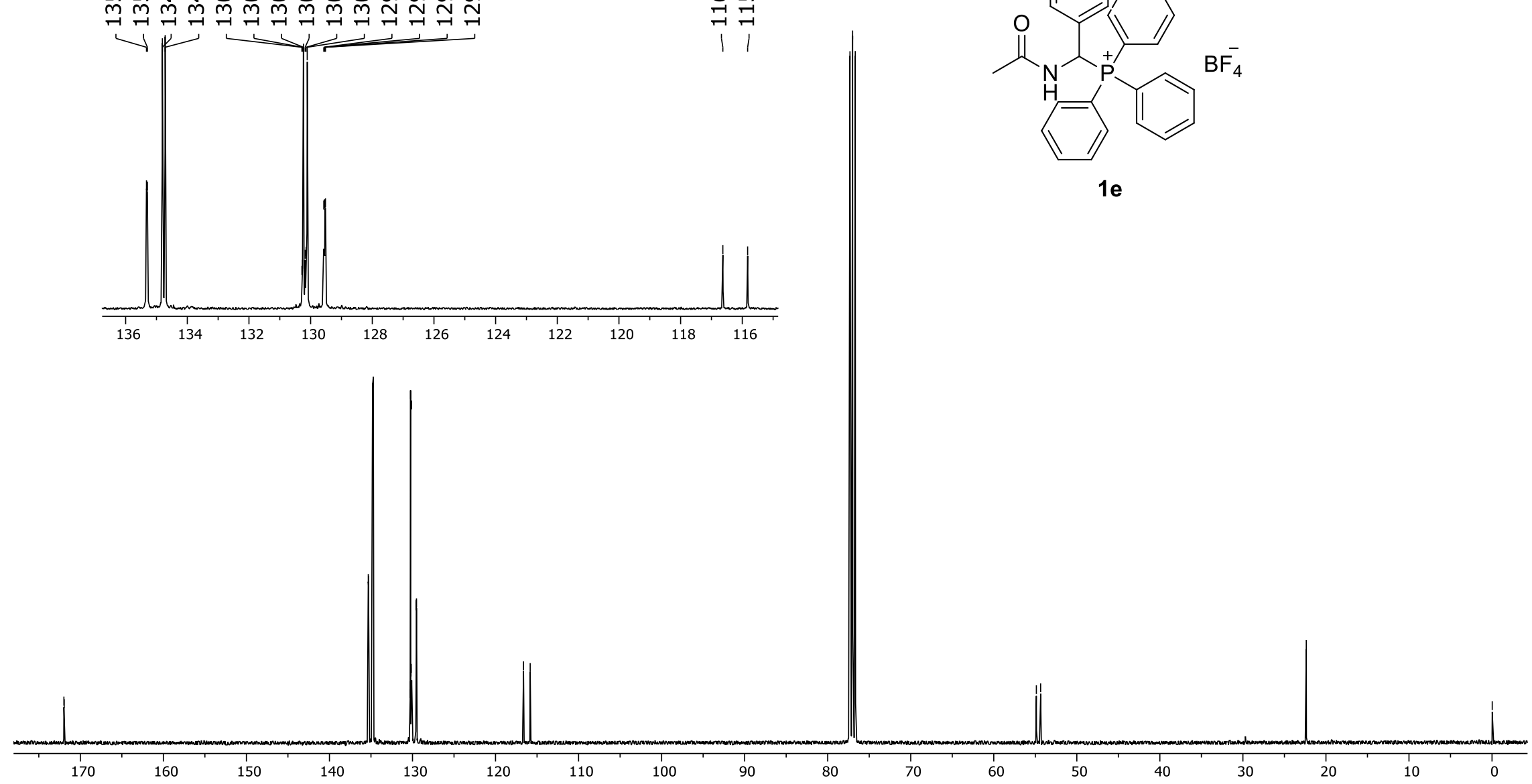

융

넏

${ }^{13} \mathrm{C}\left\{{ }^{1} \mathrm{H}\right\}$ NMR spectrum of ( $N$-acetylamino)phenylmethyltriphenylphosphonium tetrafluoroborate (1e); $100 \mathrm{MHz} / \mathrm{CDCl} 3 / \mathrm{TMS} ; \delta$ (ppm) 


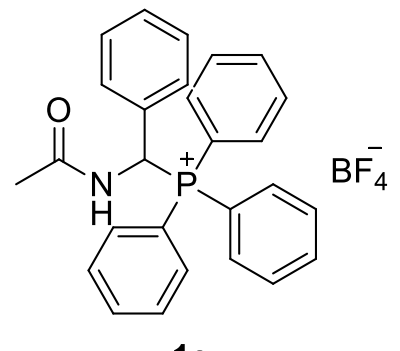

$1 e$

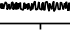

${ }^{31} \mathrm{P}$ NMR spectrum of ( $N$-acetylamino)phenylmethyltriphenylphosphonium tetrafluoroborate (1e); $161.9 \mathrm{MHz} / \mathrm{CDCl}_{3} ; \delta(\mathrm{ppm})$. 


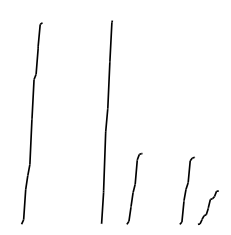

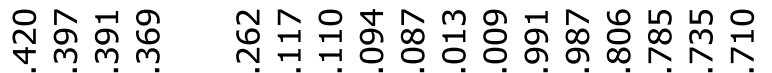

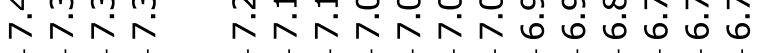

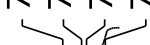

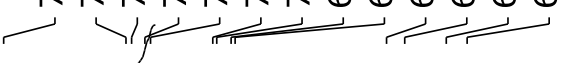
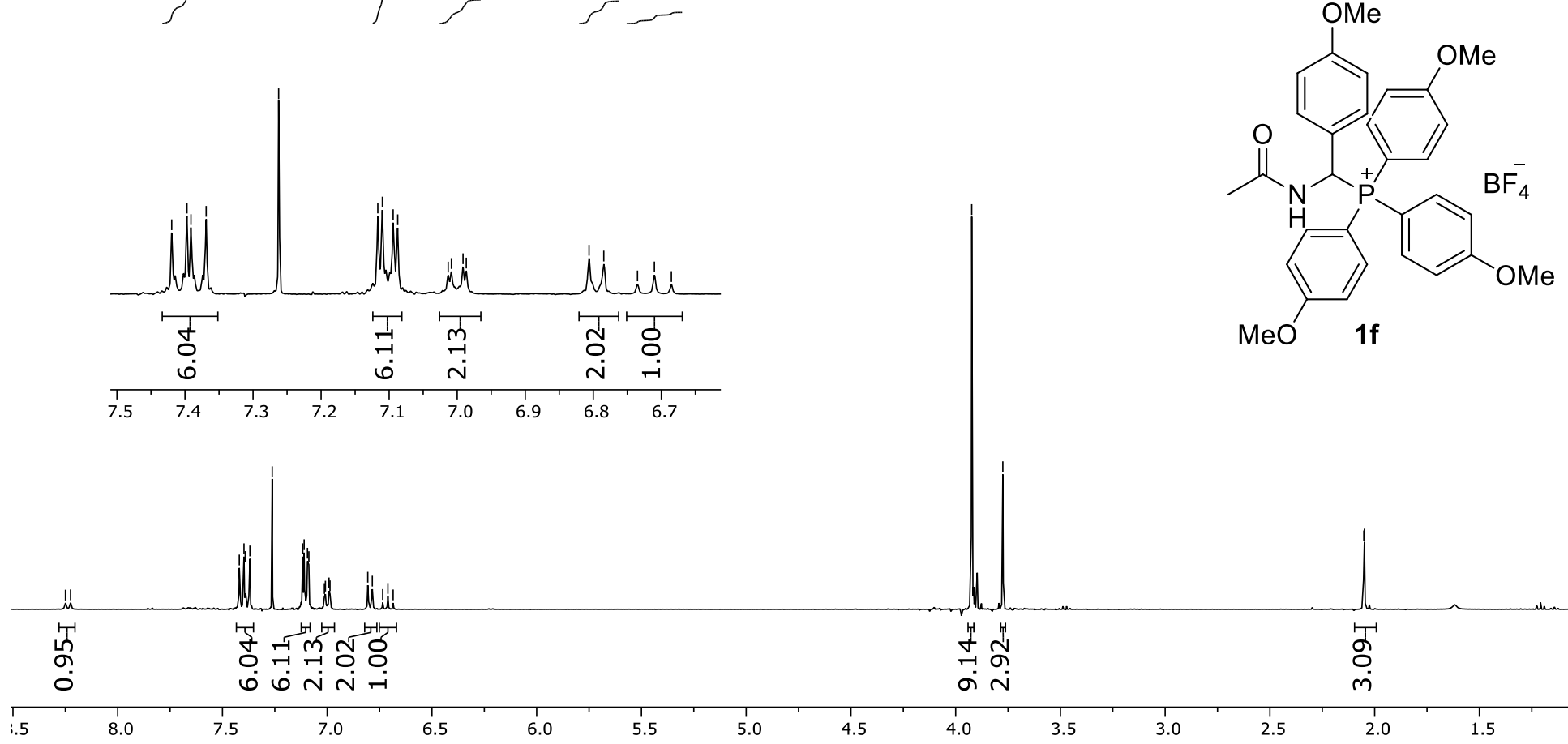

${ }^{1} \mathrm{H}$ NMR spectrum of 1-(N-acetylamino)-1-(4-metoxyphenyl)methyltris(4-metoxyphenyl)phosphonium tetrafluoroborate (1f); 400

$\mathrm{MHz} / \mathrm{CDCl}_{3} / \mathrm{TMS} ; \delta(\mathrm{ppm})$. 


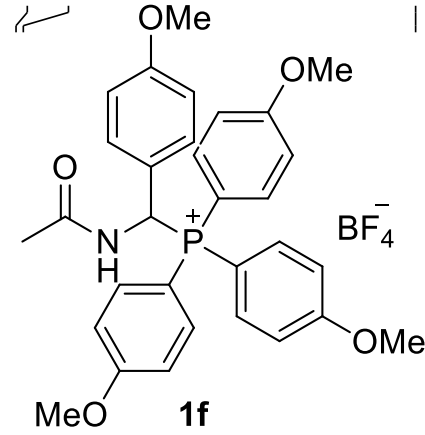

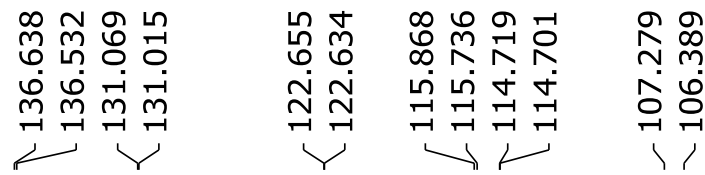
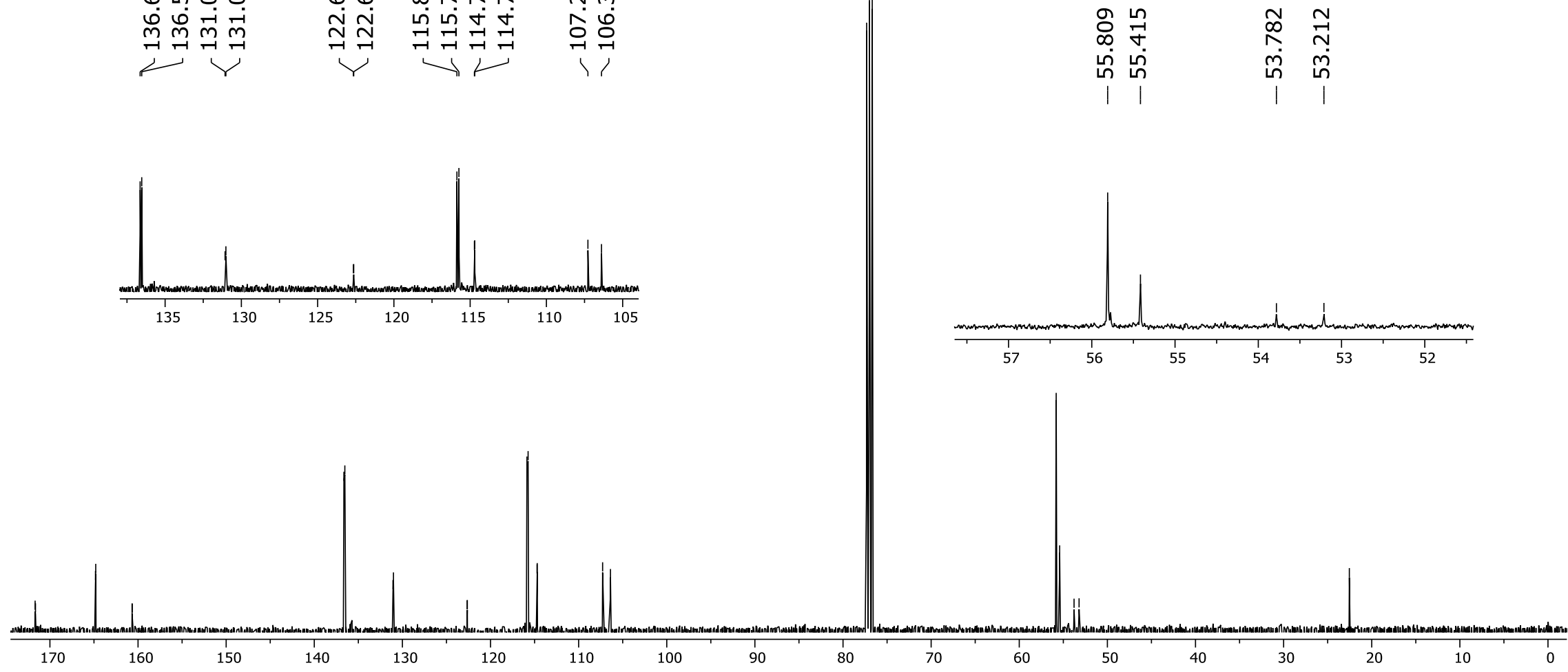

${ }^{13} \mathrm{C}\left\{{ }^{1} \mathrm{H}\right\}$ NMR spectrum of 1-(N-acetylamino)-1-(4-metoxyphenyl)methyltris(4-metoxyphenyl)phosphonium tetrafluoroborate (1f); 100 $\mathrm{MHz} / \mathrm{CDCl}_{3} / \mathrm{TMS} ; \delta$ (ppm). 


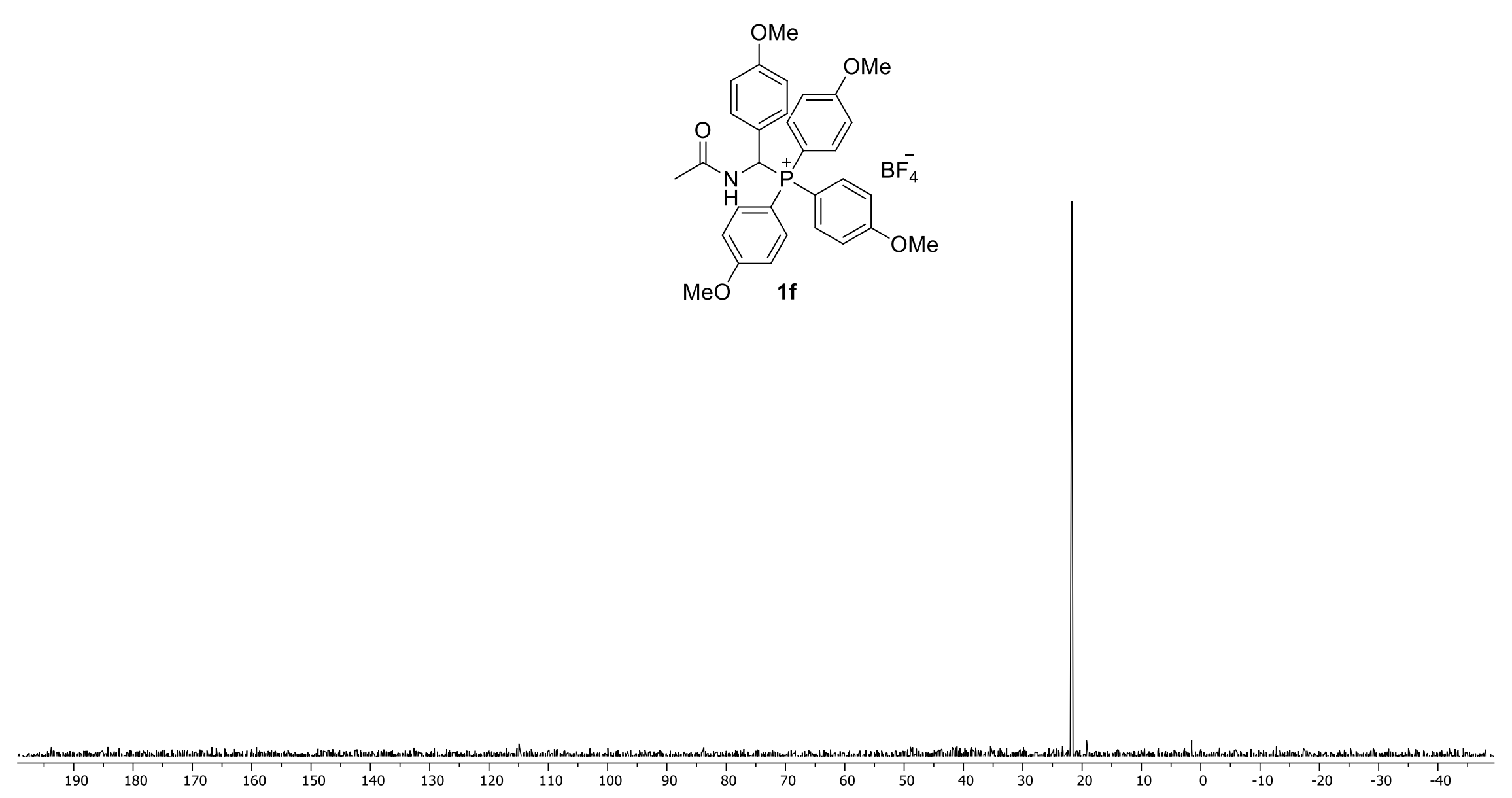

${ }^{31} \mathrm{P}$ NMR spectrum of 1-(N-acetylamino)-1-(4-metoxyphenyl)methyltris(4-metoxyphenyl)phosphonium tetrafluoroborate (1f); 161.9 MHz/CDCl ${ }_{3}$; $\delta(\mathrm{ppm})$ 


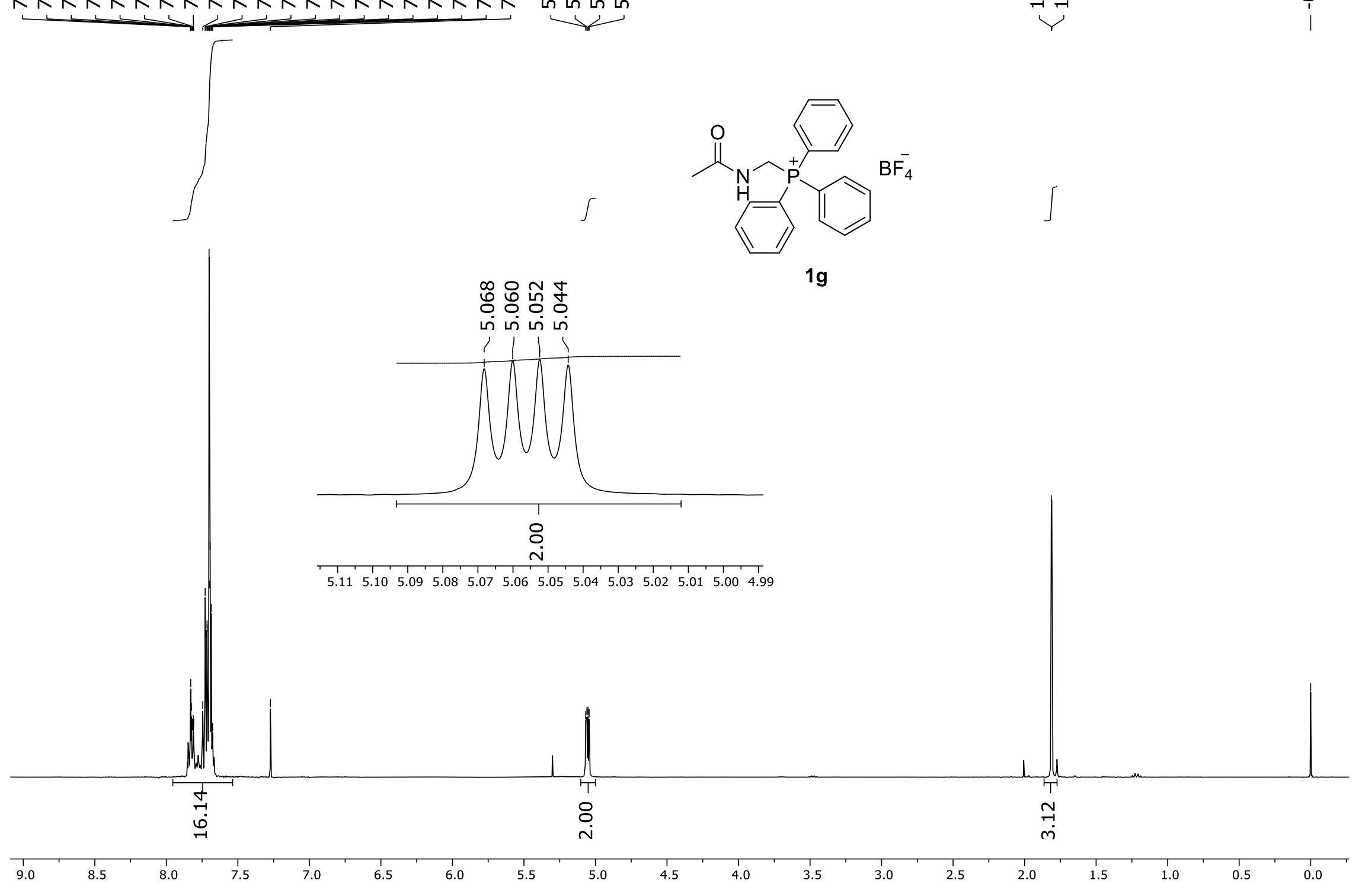

${ }^{1} \mathrm{H}$ NMR spectrum of ( $N$-acetylamino)methyltriphenylphosphonium tetrafluoroborate (1g); $400 \mathrm{MHz} / \mathrm{CDCl}_{3} / \mathrm{TMS} ; \delta$ (ppm). 

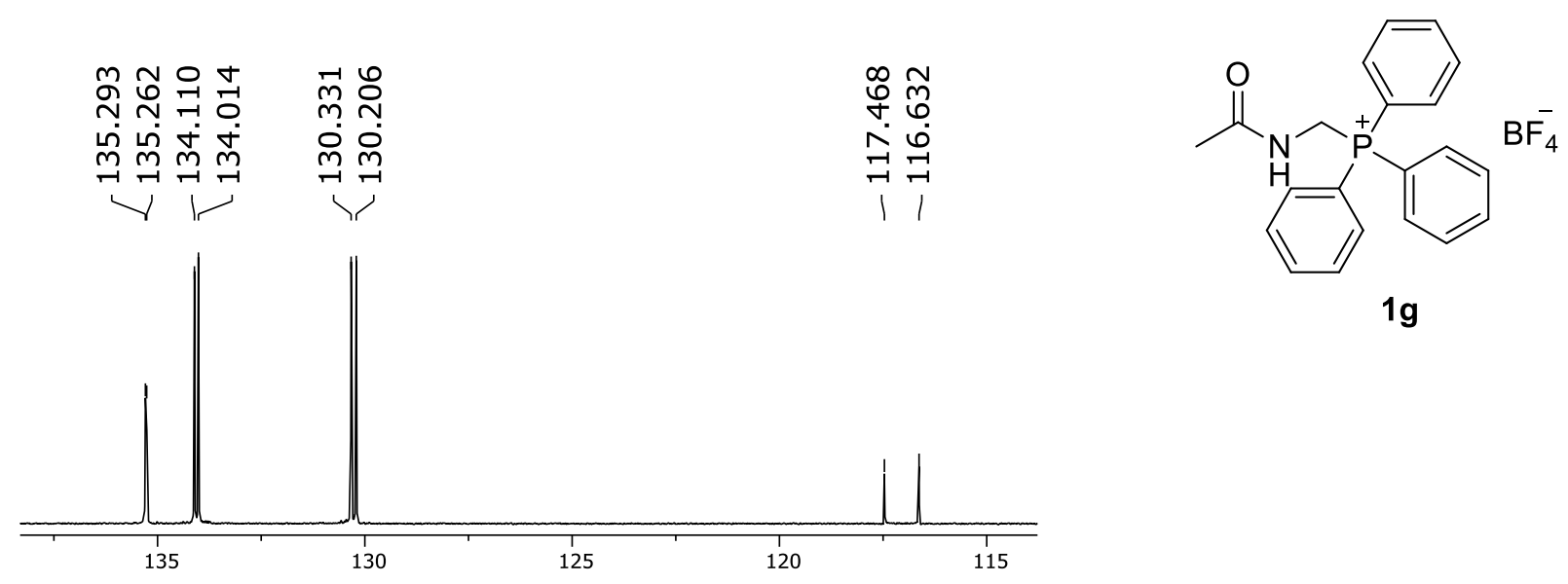

$1 \mathrm{~g}$

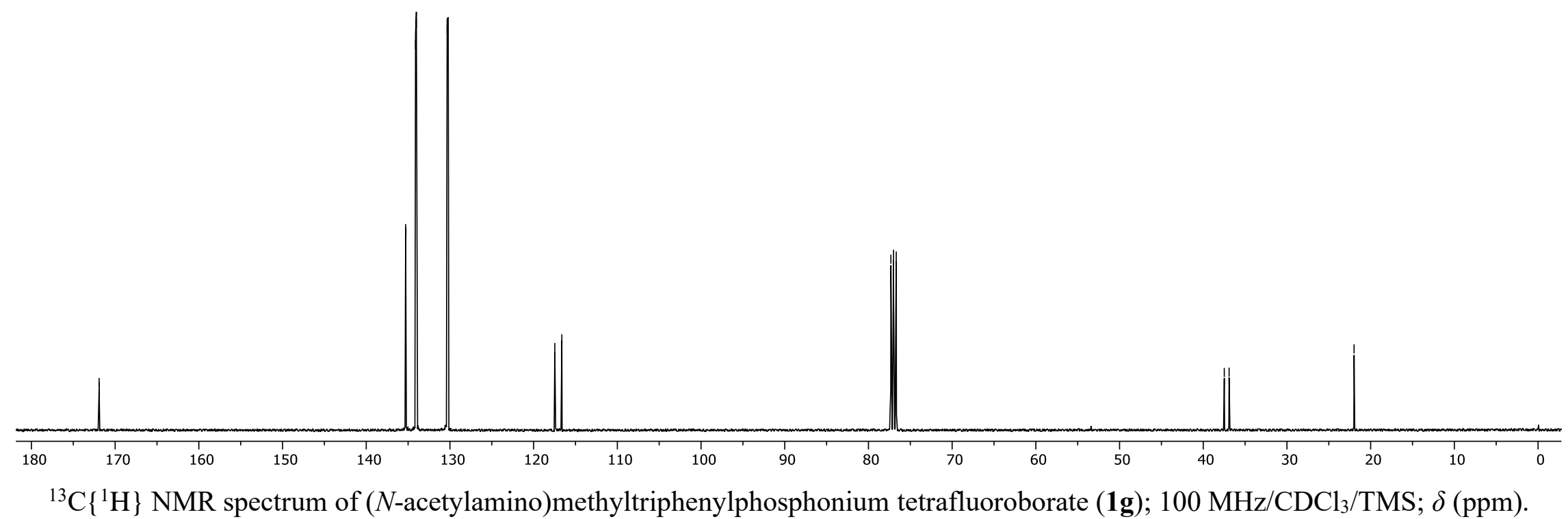




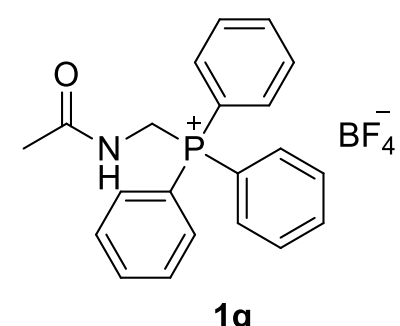

$1 \mathrm{~g}$

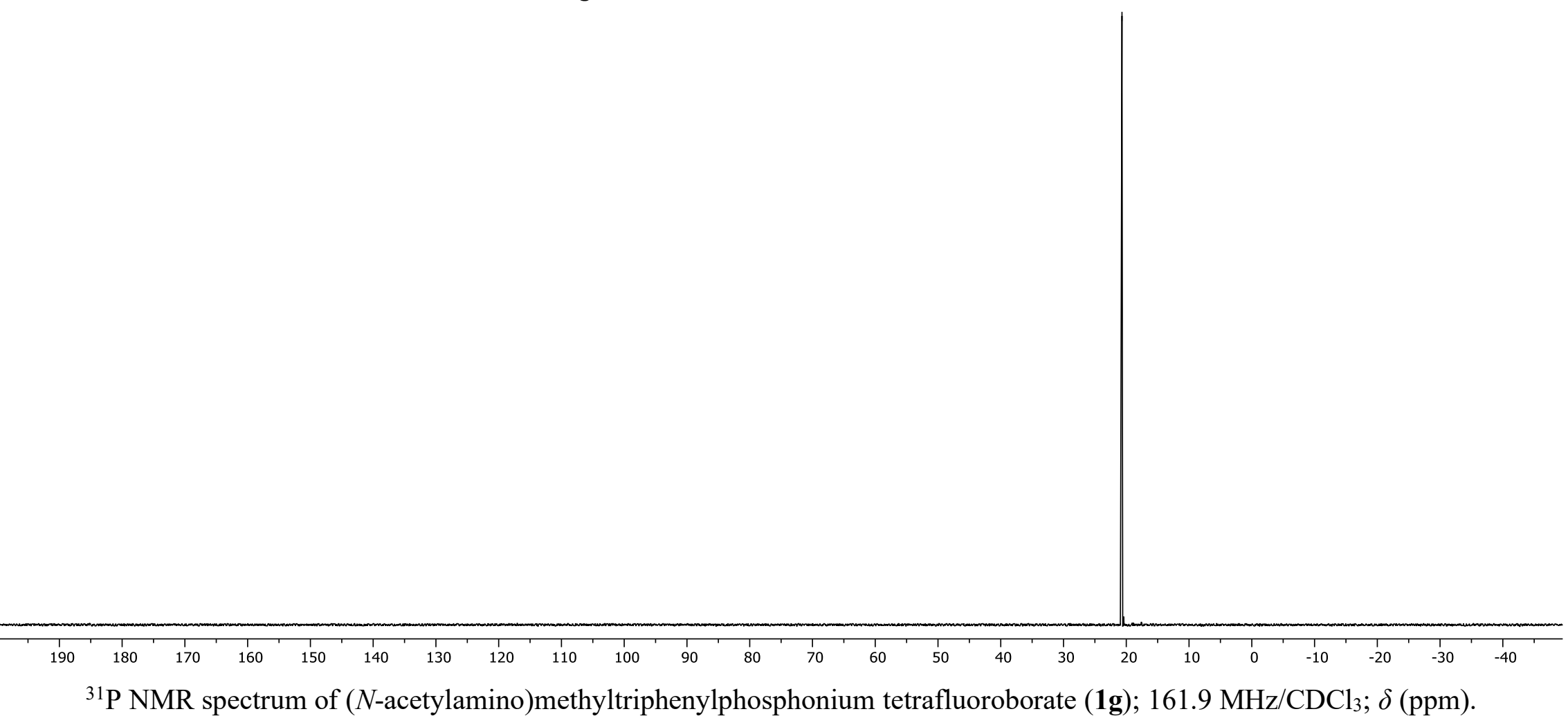




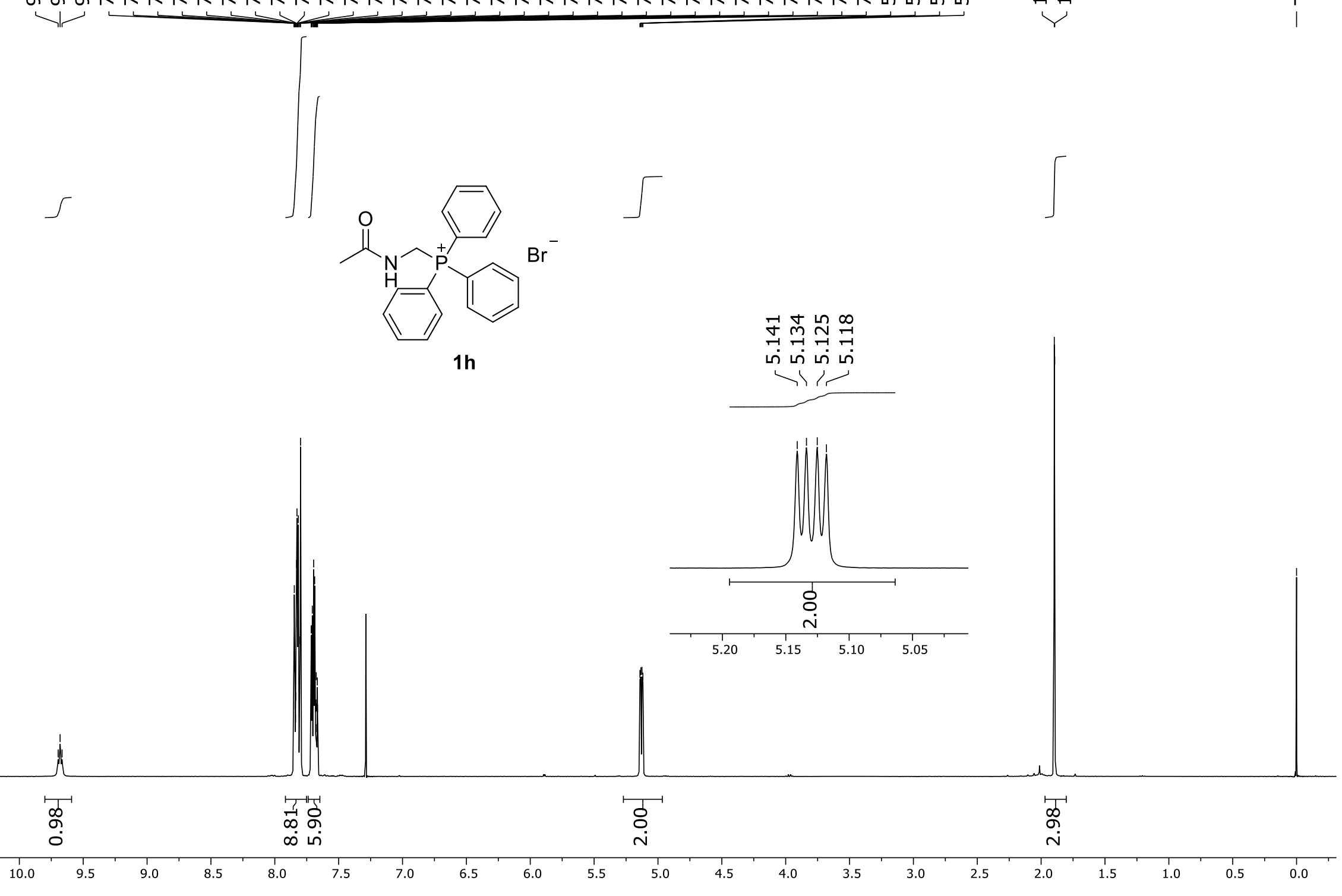

${ }^{1} \mathrm{H}$ NMR spectrum of ( $N$-acetylamino)methyltriphenylphosphonium bromide (1h); $400 \mathrm{MHz} / \mathrm{CDCl}_{3} / \mathrm{TMS} ; \delta$ (ppm). 


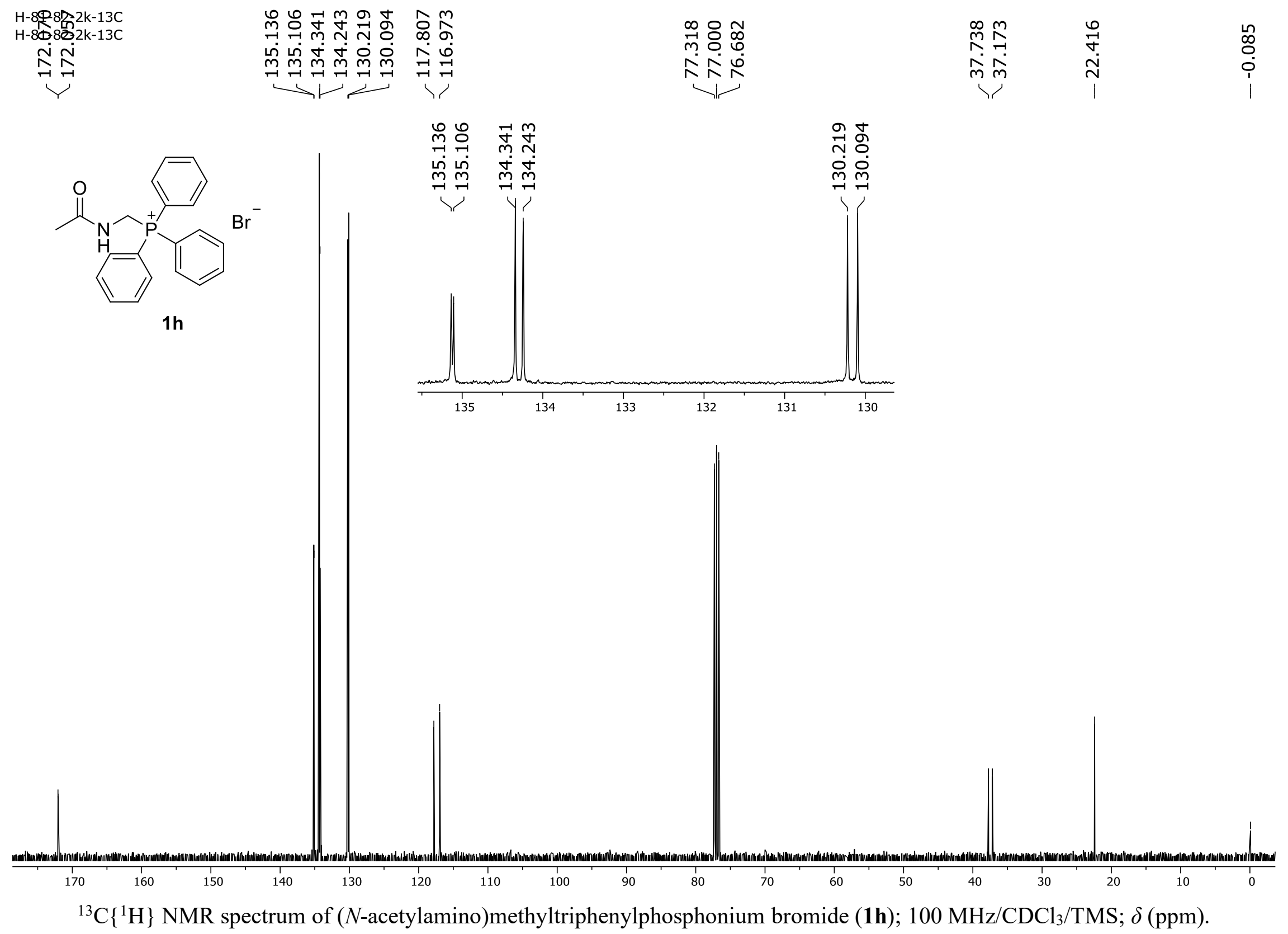




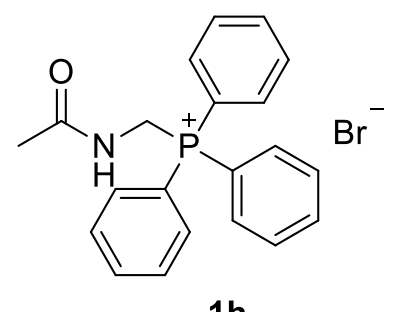

1h

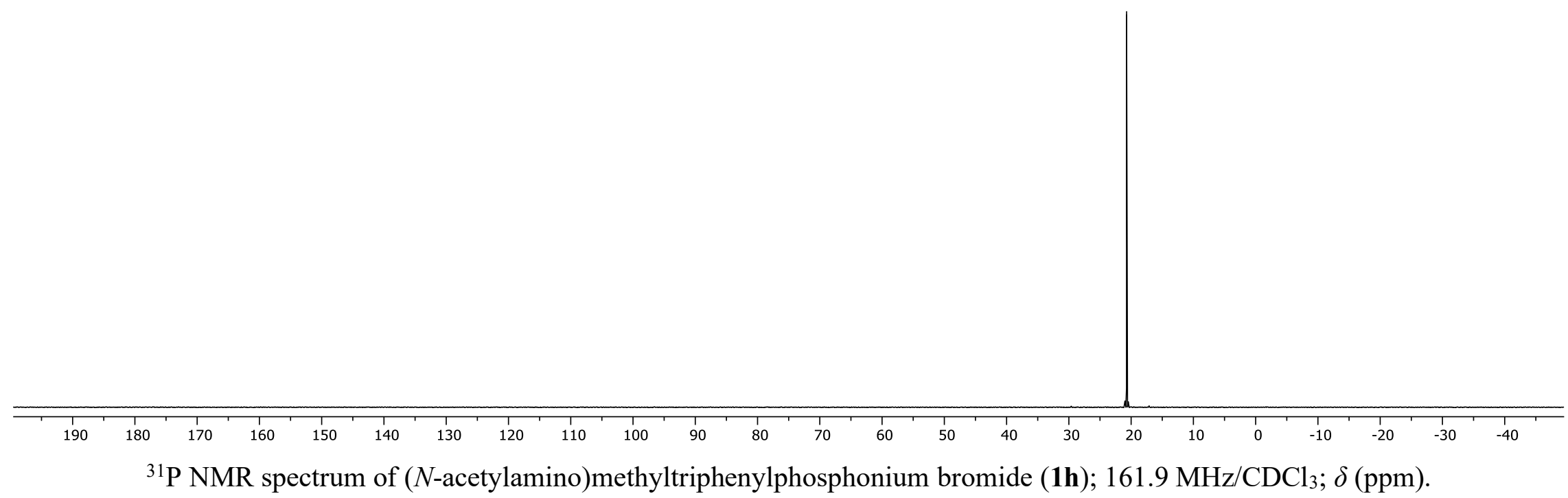




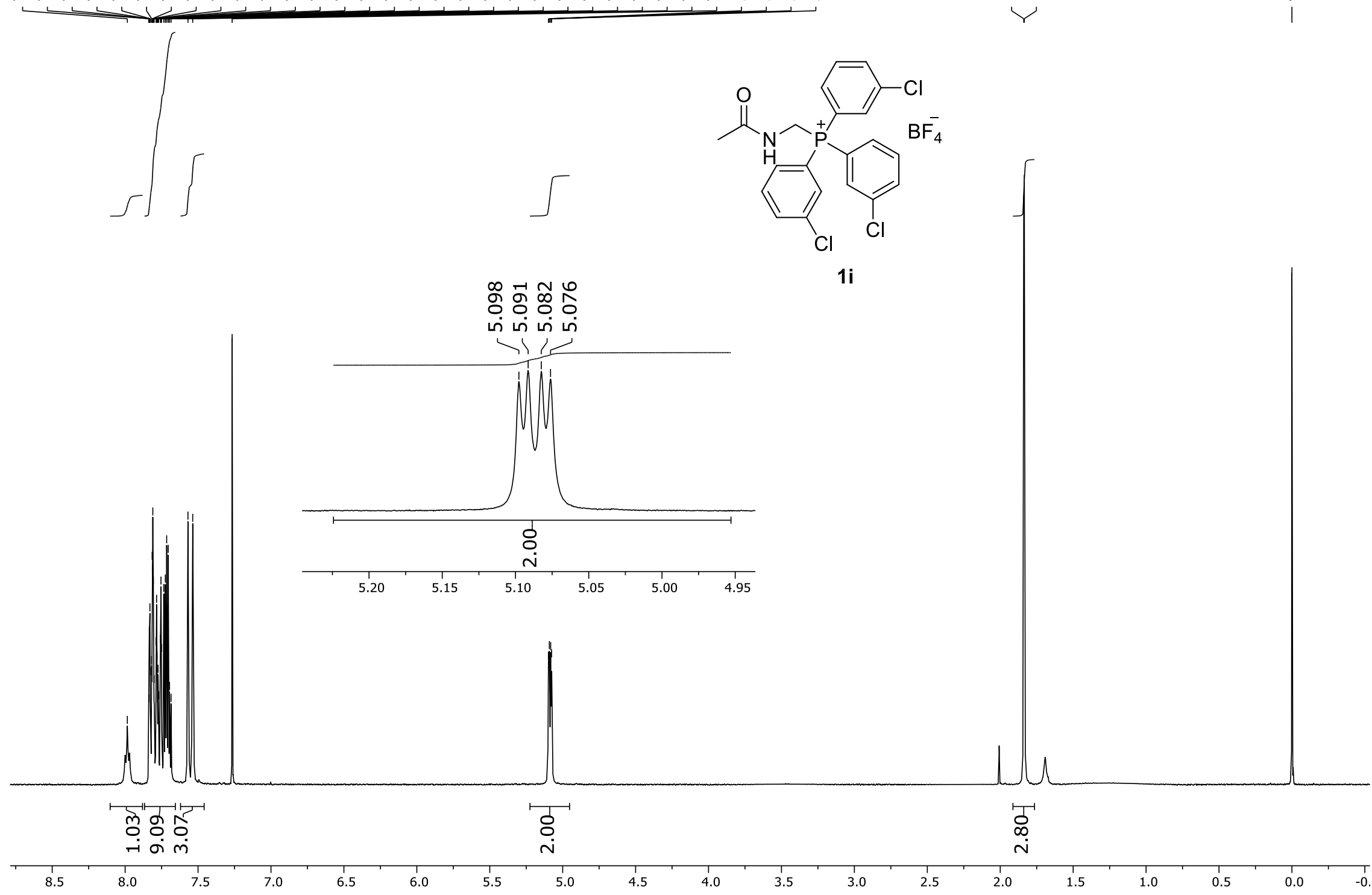

${ }^{1} \mathrm{H}$ NMR spectrum of ( $N$-acetylamino)methyltris(3-chlorophenyl)phosphonium tetrafluoroborate (1i); $400 \mathrm{MHz} / \mathrm{CDCl} 3 / \mathrm{TMS} ; \delta$ (ppm). 
H-83-表-永Cws H-83-2k-

촉

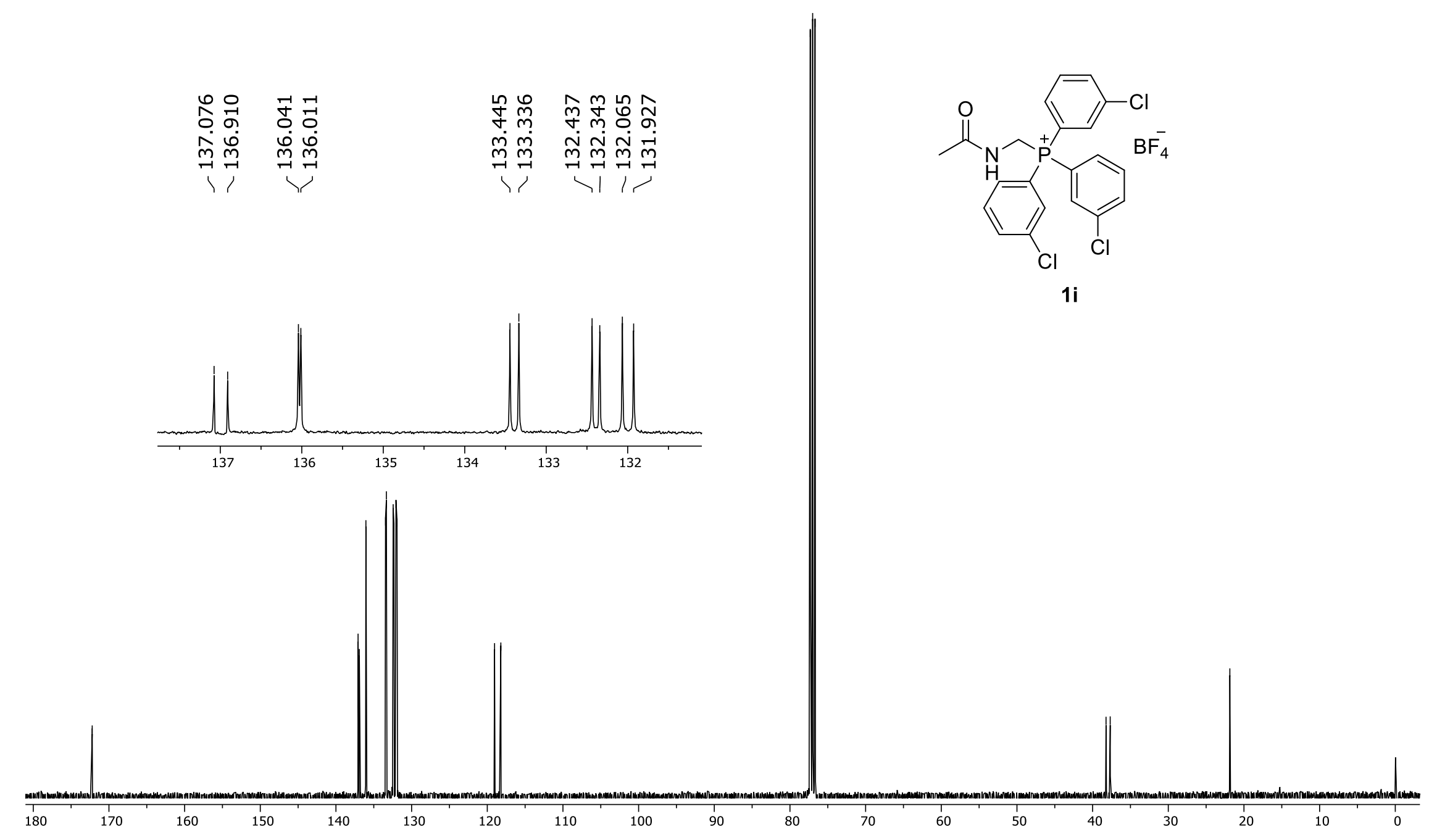

${ }^{13} \mathrm{C}\left\{{ }^{1} \mathrm{H}\right\}$ NMR spectrum of ( $N$-acetylamino)methyltris(3-chlorophenyl)phosphonium tetrafluoroborate (1i); $100 \mathrm{MHz} / \mathrm{CDCl}_{3} / \mathrm{TMS} ; \delta$ (ppm). 


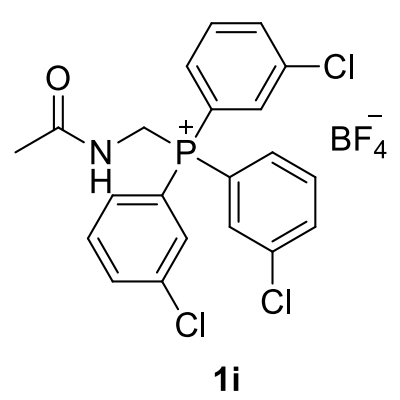

$1 i$

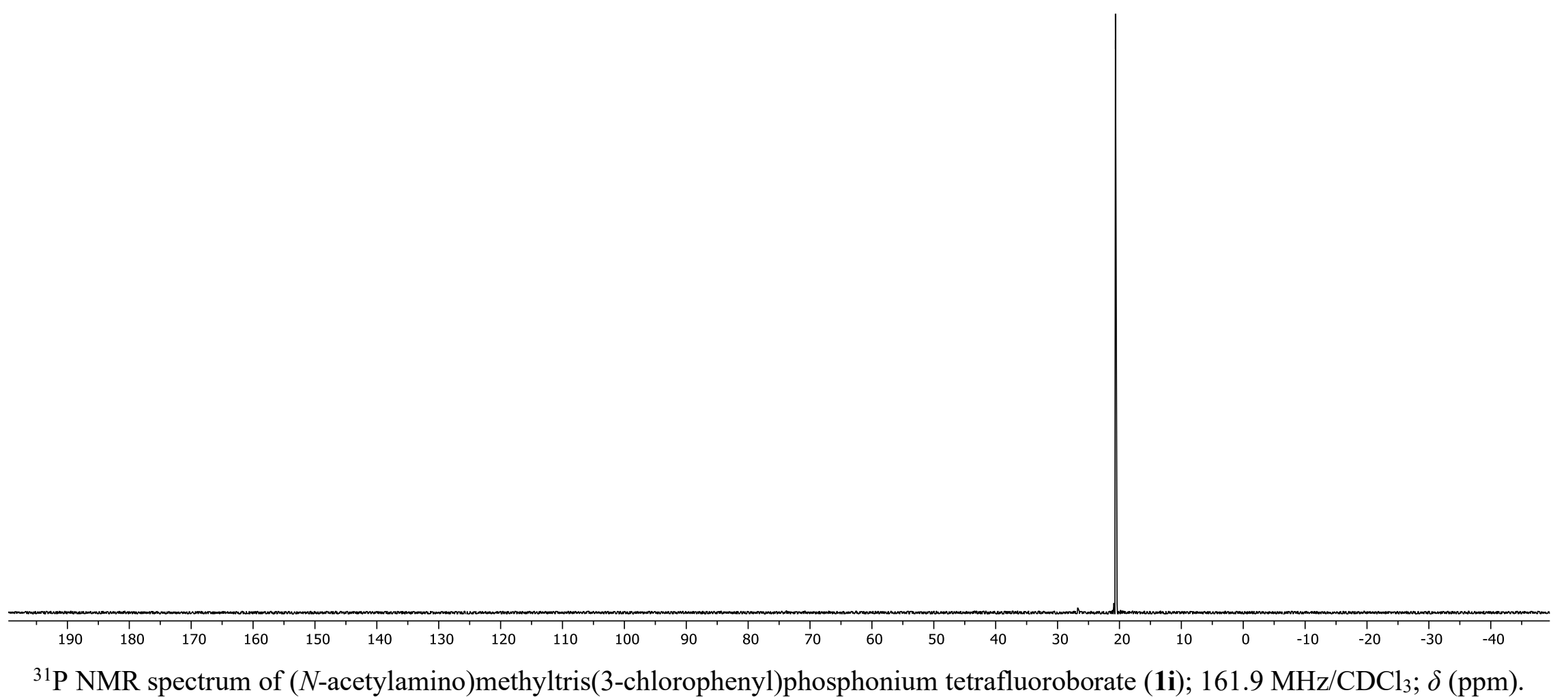




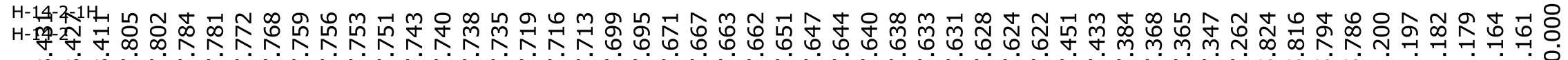

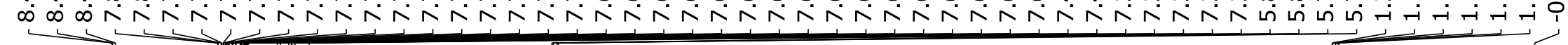

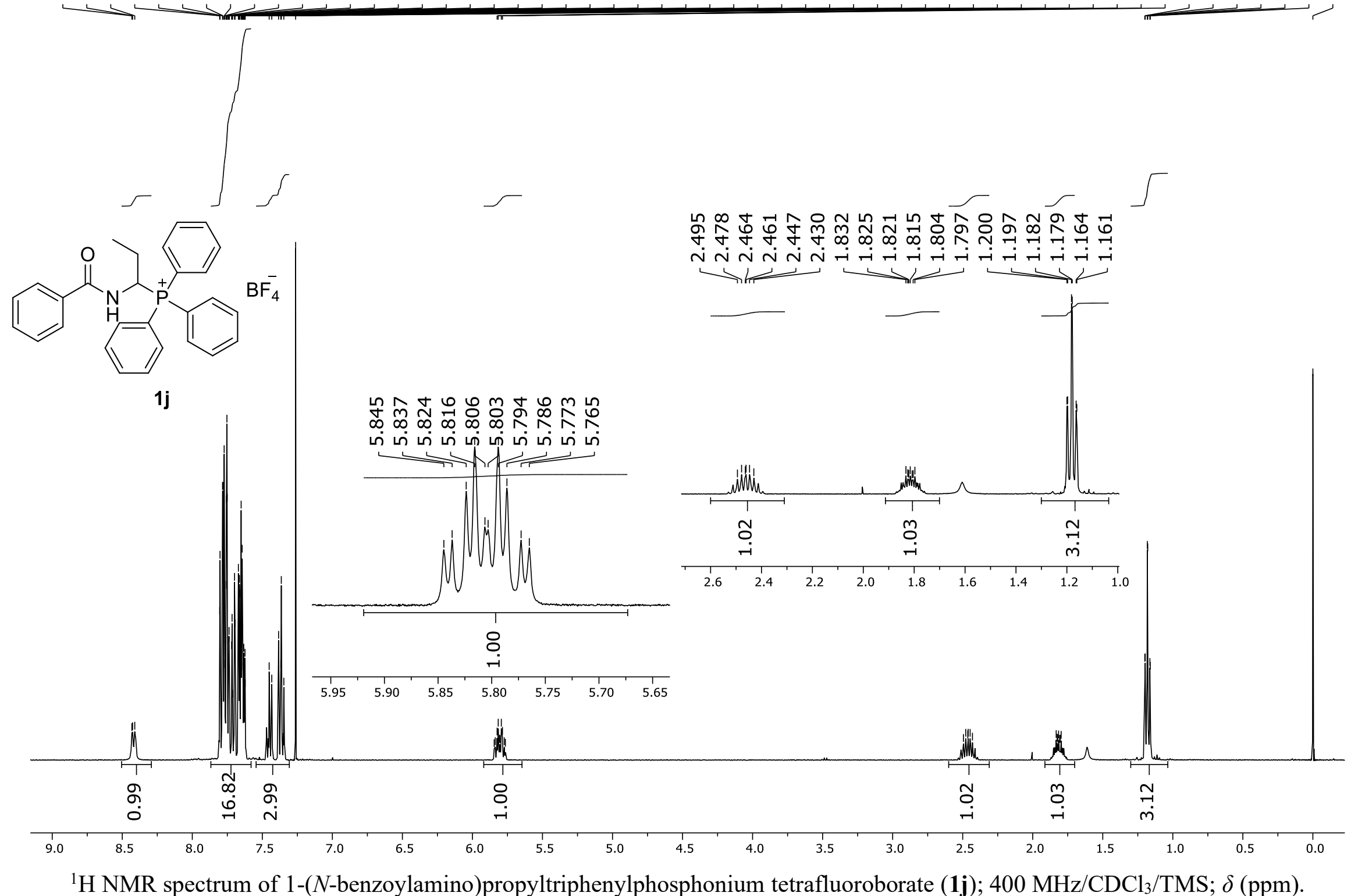

${ }^{1} \mathrm{H}$ NMR spectrum of 1-( $N$-benzoylamino)propyltriphenylphosphonium tetrafluoroborate (1j); $400 \mathrm{MHz} / \mathrm{CDCl} 3 / \mathrm{TMS} ; \delta$ (ppm). 


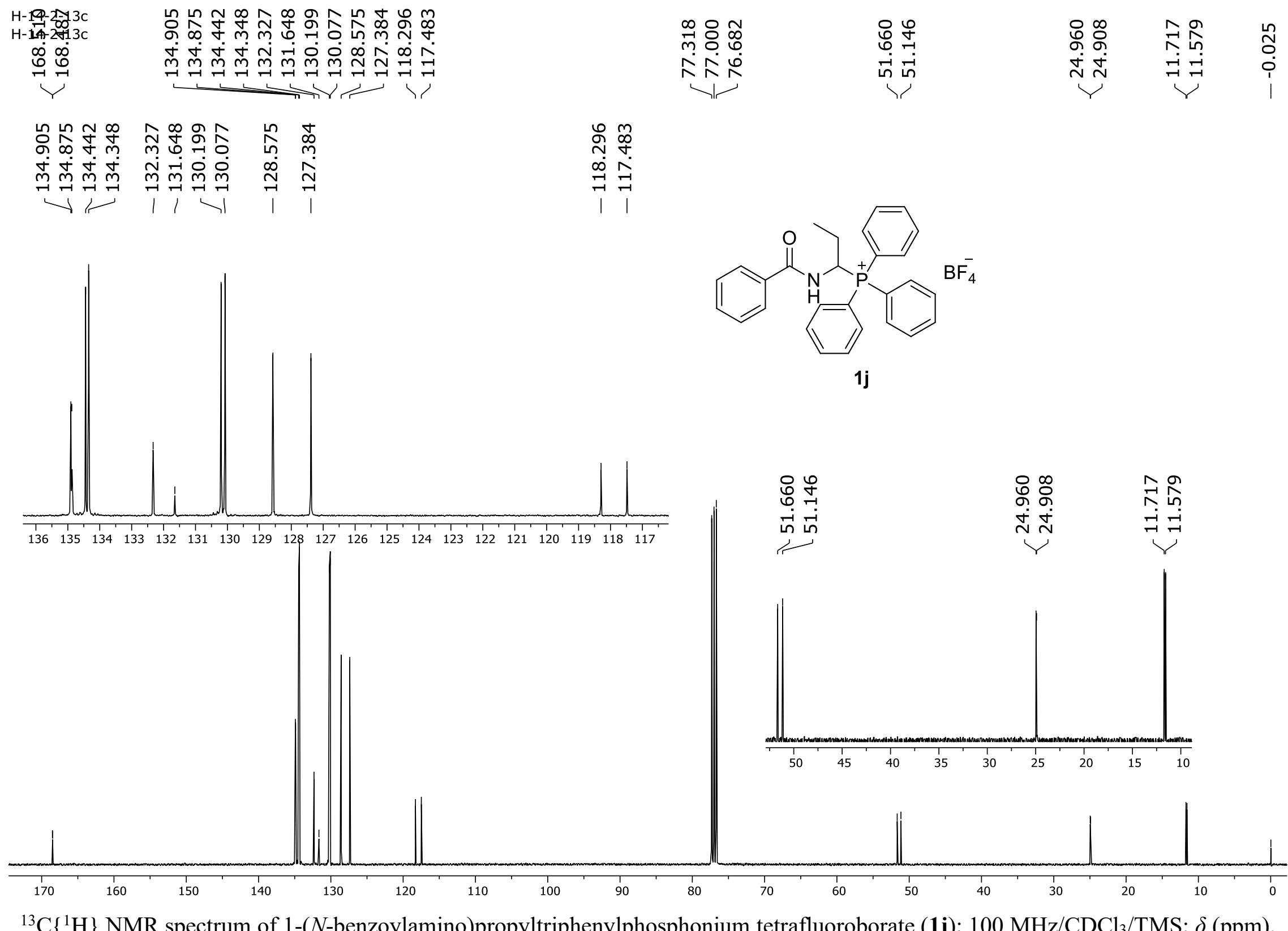

${ }^{13} \mathrm{C}\left\{{ }^{1} \mathrm{H}\right\}$ NMR spectrum of 1-(N-benzoylamino)propyltriphenylphosphonium tetrafluoroborate (1j); $100 \mathrm{MHz} / \mathrm{CDCl} / \mathrm{TMS} ; \delta$ (ppm). 

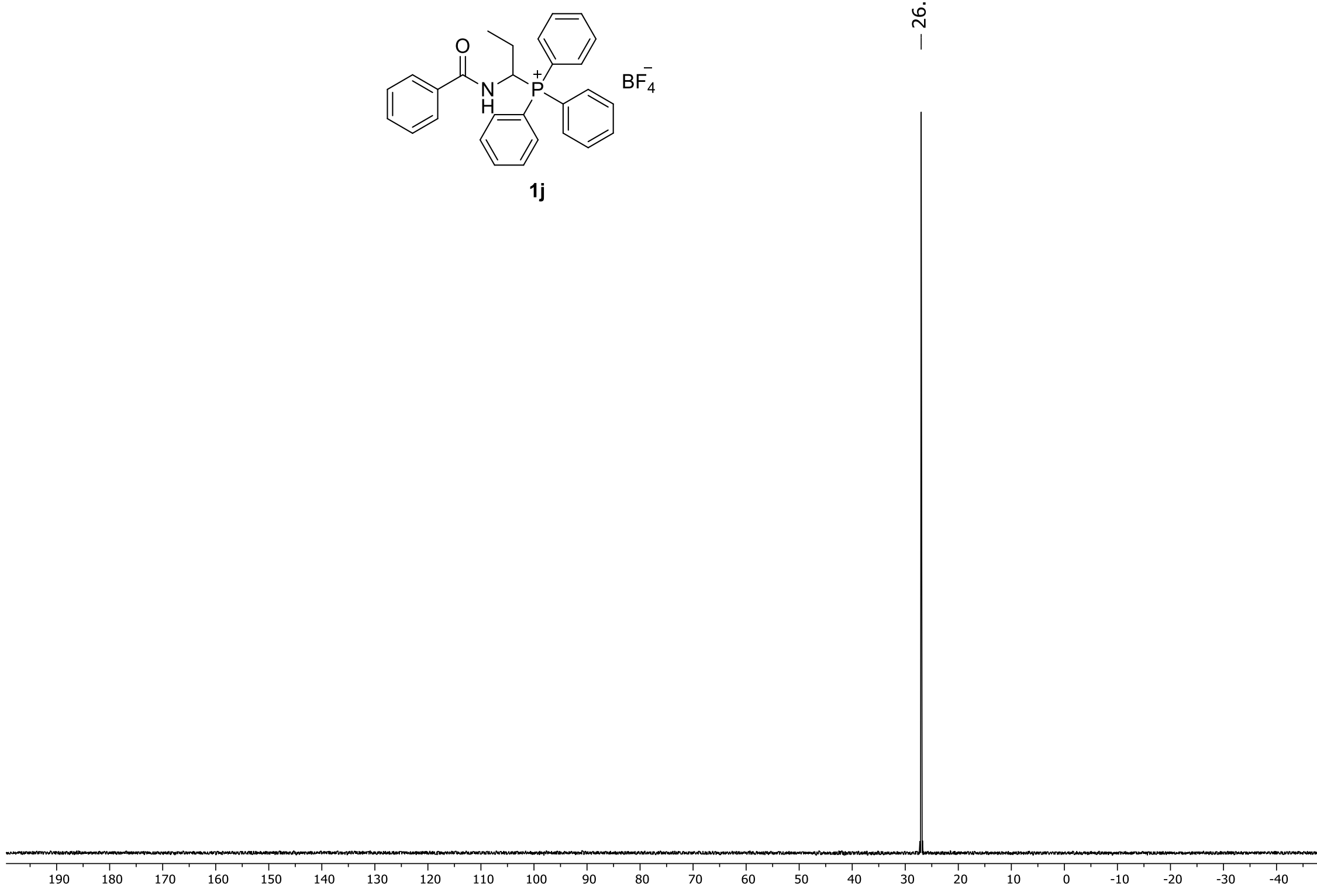

${ }^{31} \mathrm{P}$ NMR spectrum of 1-( $N$-benzoylamino)propyltriphenylphosphonium tetrafluoroborate $(\mathbf{1 j}) ; 161.9 \mathrm{MHz} / \mathrm{CDCl}_{3} ; \delta(\mathrm{ppm})$. 


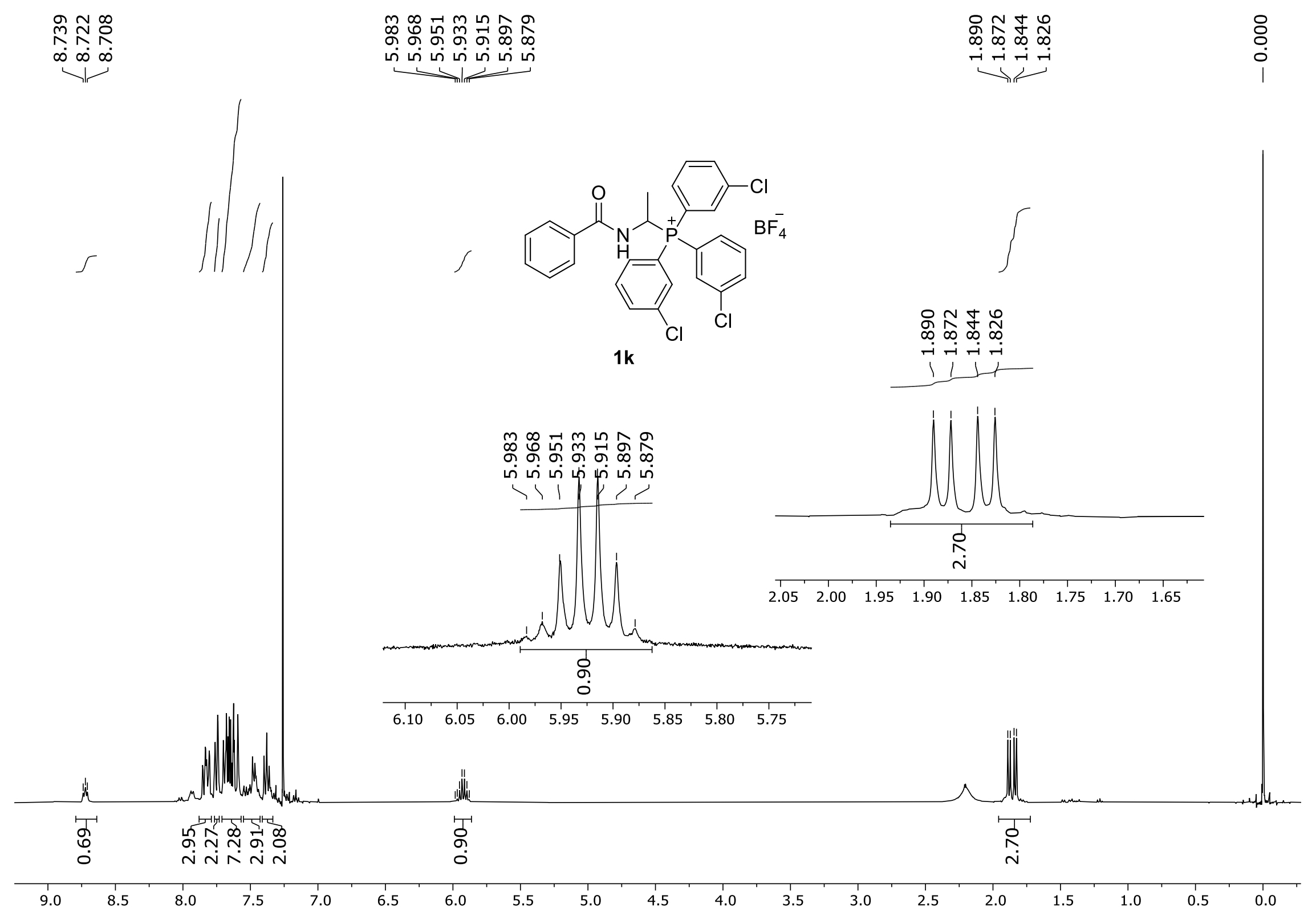

${ }^{1} \mathrm{H}$ NMR spectrum of 1-( $N$-benzoylamino)ethyltris(3-chlorophenyl)phosphonium tetrafluoroborate (1k); $400 \mathrm{MHz} / \mathrm{CDCl} / \mathrm{TMS} ; \delta$ (ppm). 

mim

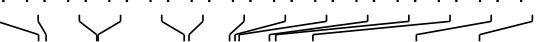

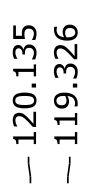
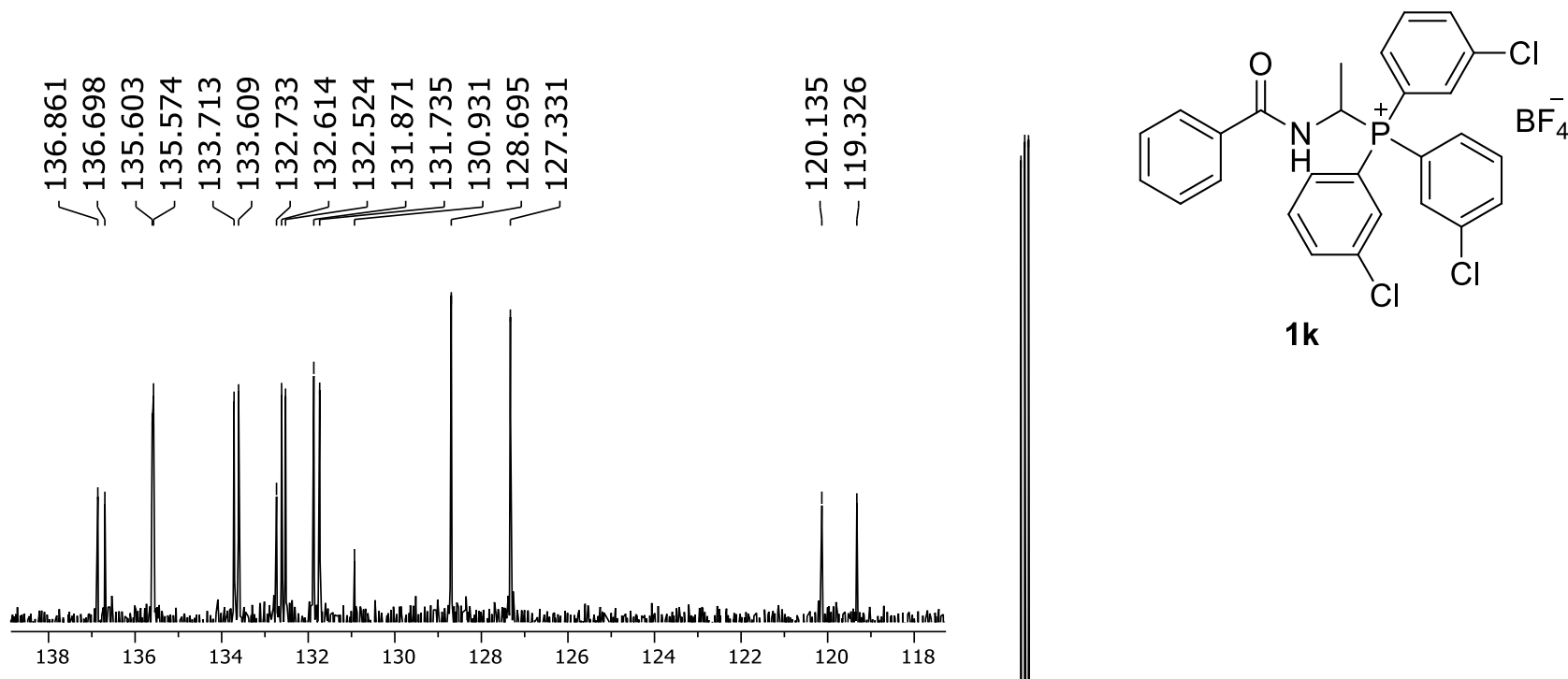

$1 \mathrm{k}$

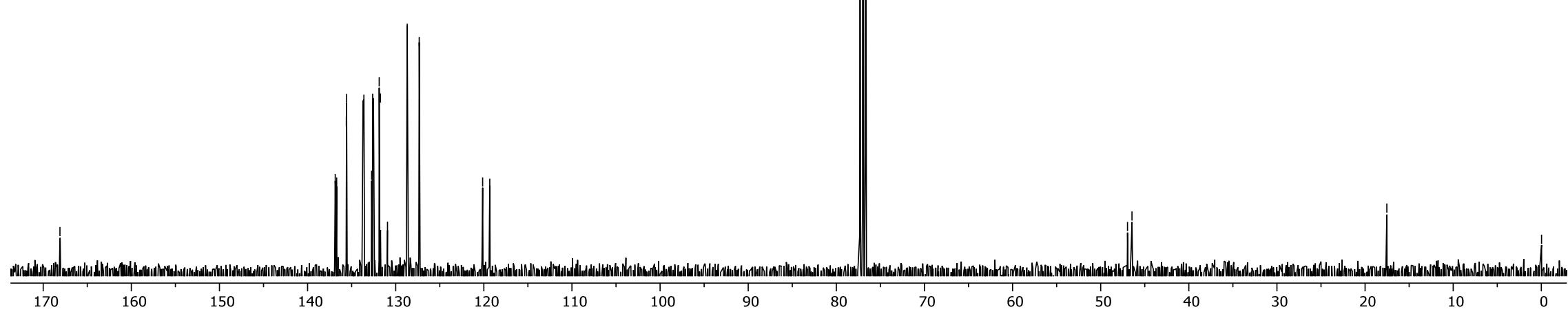

${ }^{13} \mathrm{C}\left\{{ }^{1} \mathrm{H}\right\}$ NMR spectrum of 1-(N-benzoylamino)ethyltris(3-chlorophenyl)phosphonium tetrafluoroborate (1k); $100 \mathrm{MHz} / \mathrm{CDCl} 3 / \mathrm{TMS} ; \delta$ (ppm). 


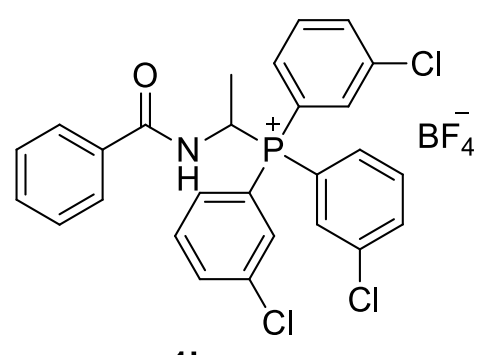

$1 k$

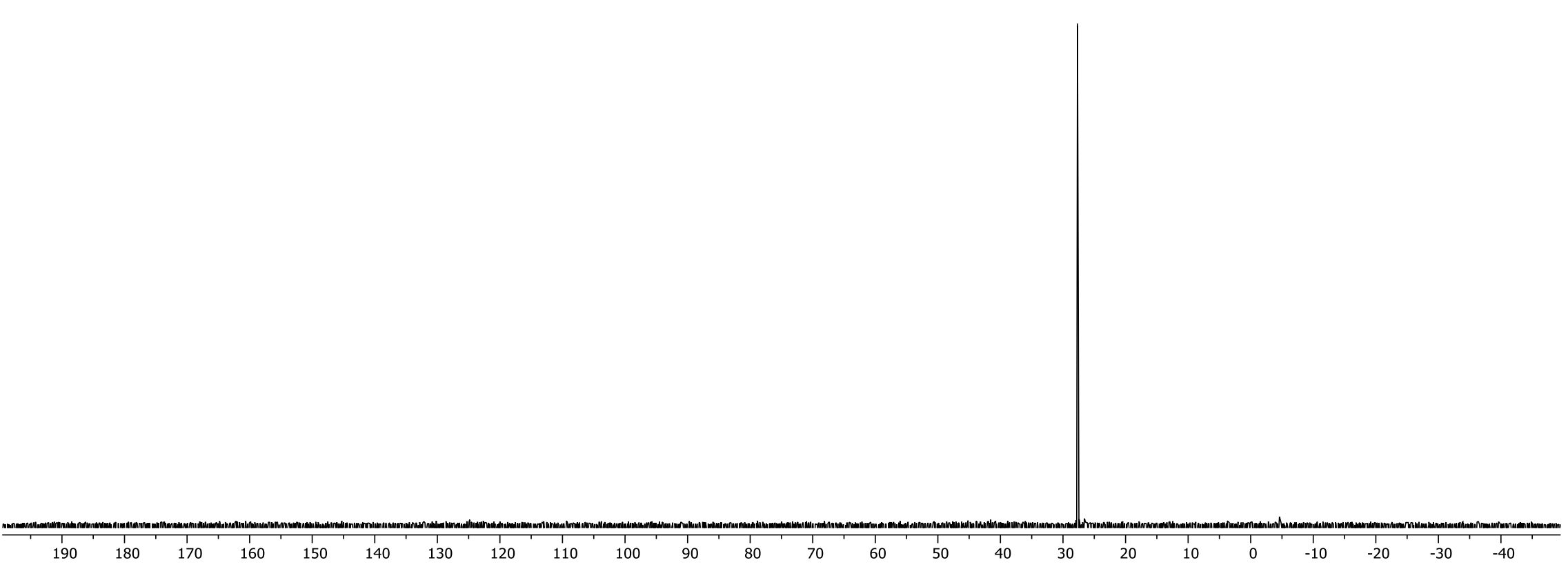

${ }^{31} \mathrm{P}$ NMR spectrum of 1 -( $N$-benzoylamino)ethyltris(3-chlorophenyl)phosphonium tetrafluoroborate (1k); $161.9 \mathrm{MHz} / \mathrm{CDCl}_{3} ; \delta(\mathrm{ppm})$. 

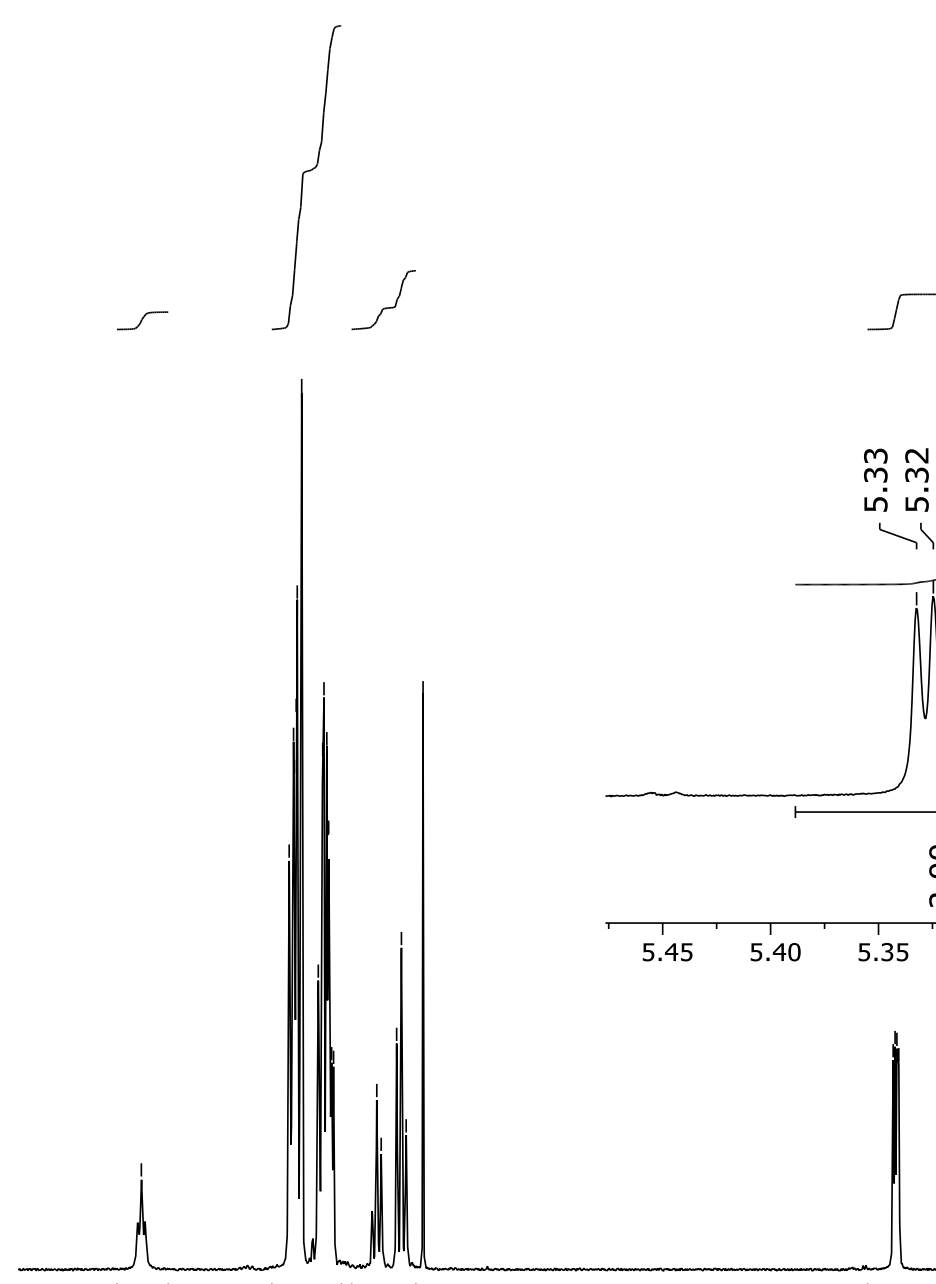

m. $\tilde{m} \tilde{m} \bar{m}$

เ่

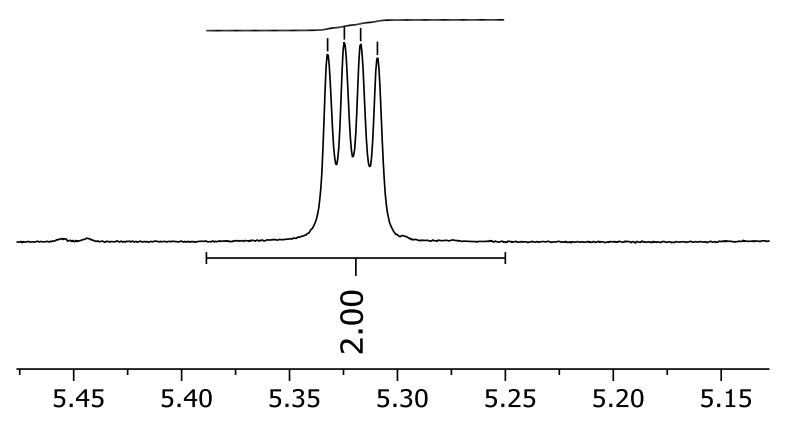

$5.40 \quad 5.35$

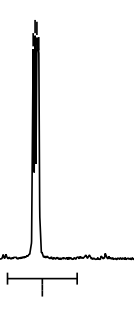

$\stackrel{\circ}{\sim}$

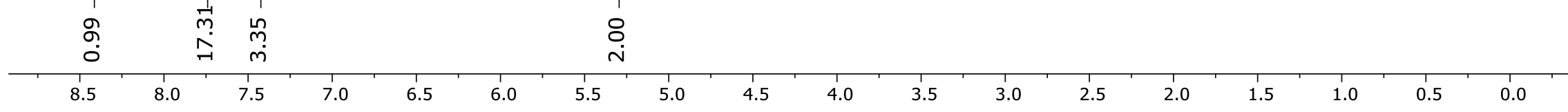

${ }^{1} \mathrm{H}$ NMR spectrum of ( $N$-benzoylamino)methyltriphenylphosphonium tetrafluoroborate (11); $400 \mathrm{MHz} / \mathrm{CDCl}_{3} / \mathrm{TMS} ; \delta$ (ppm). 


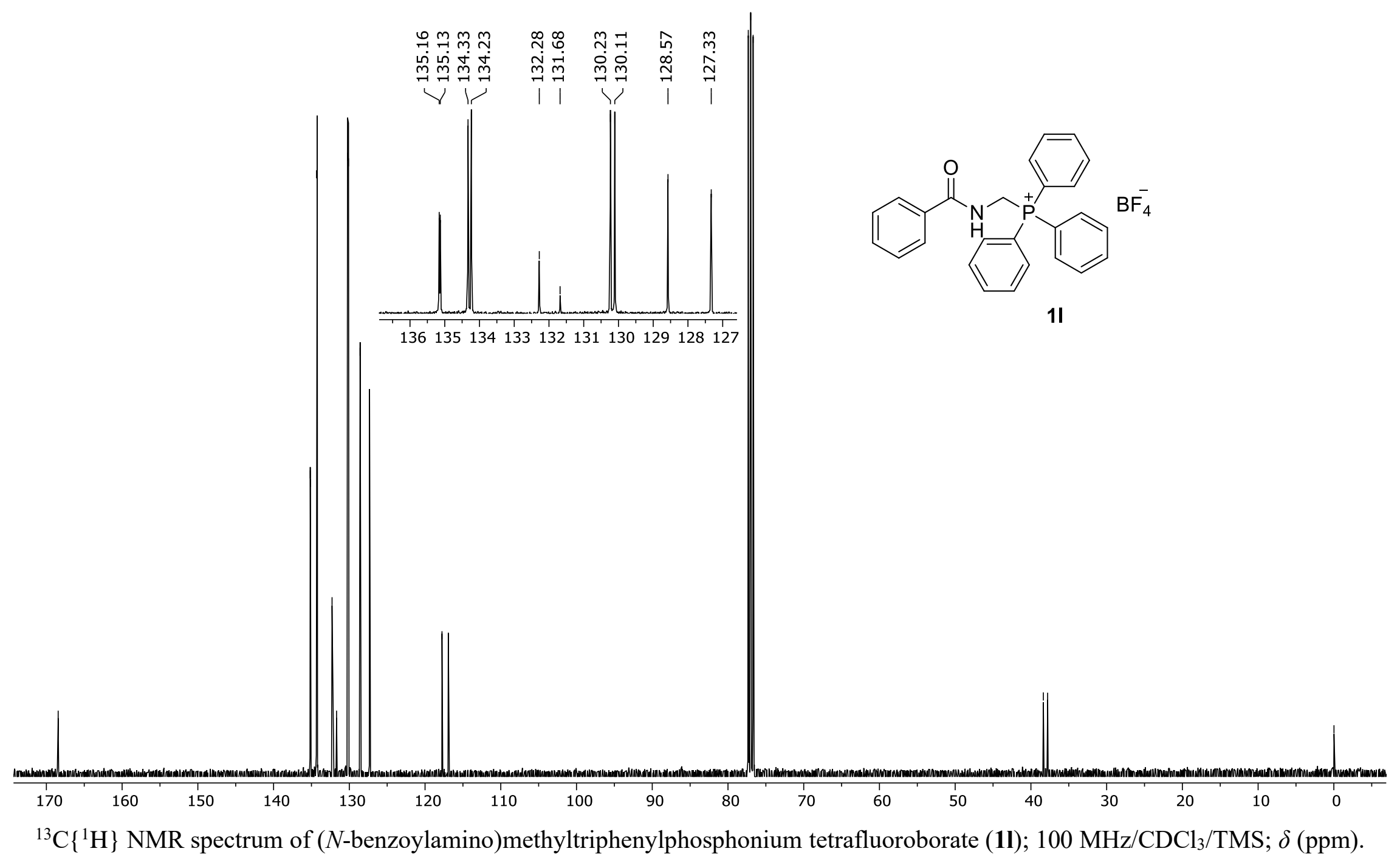



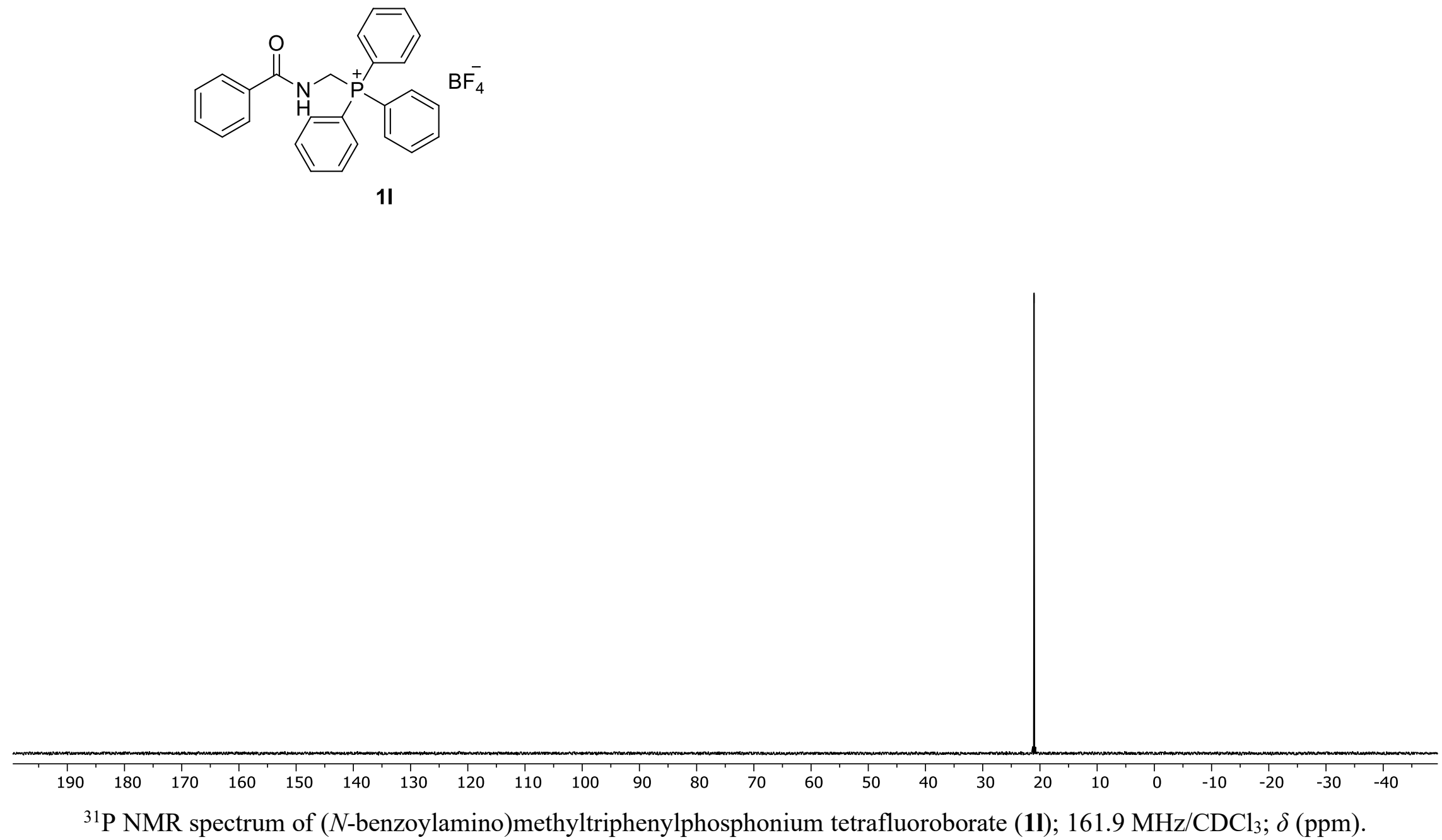
$\int$

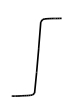

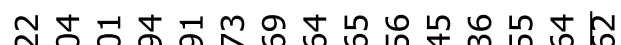

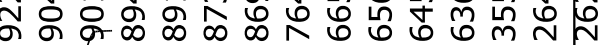

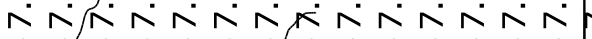
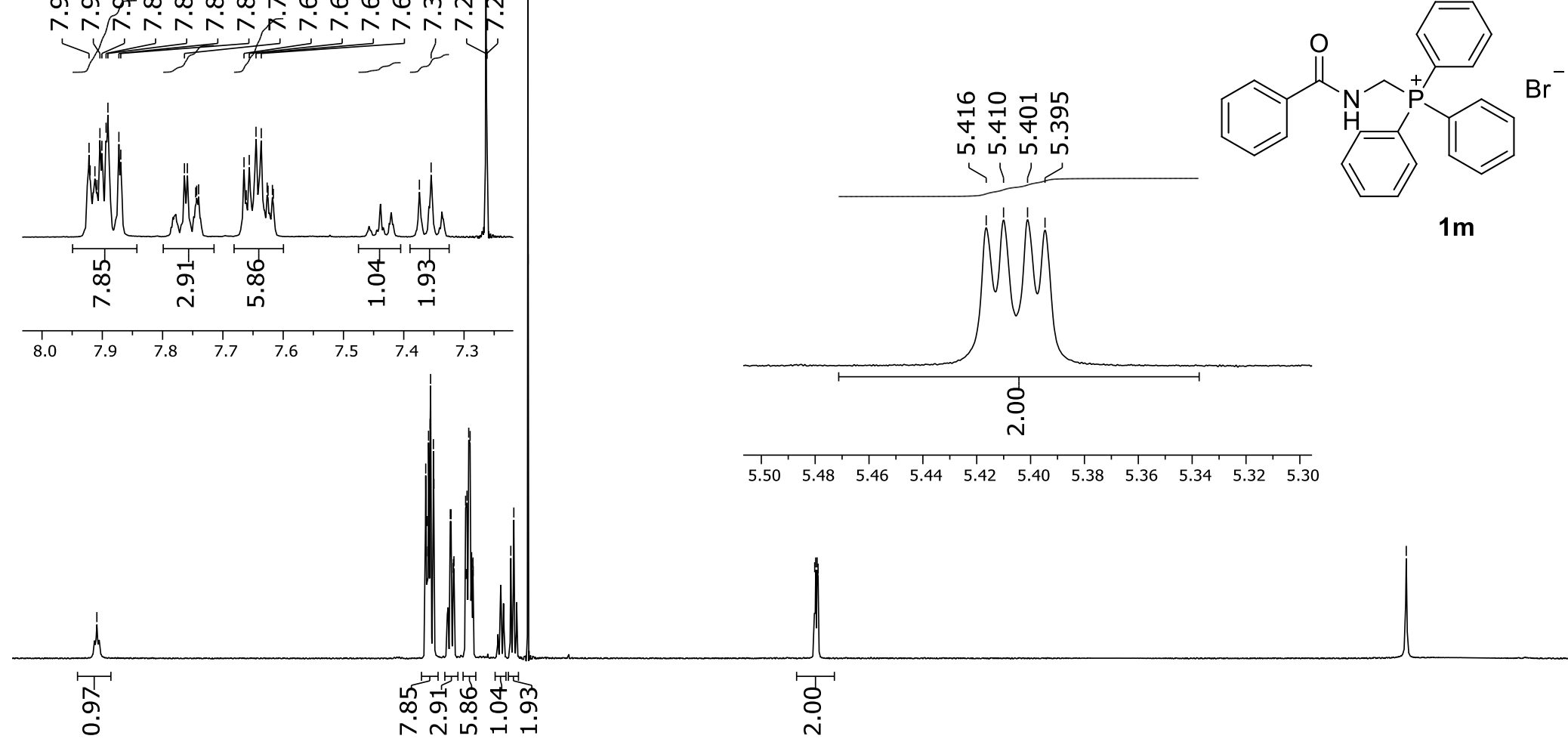

$1 \mathrm{~m}$
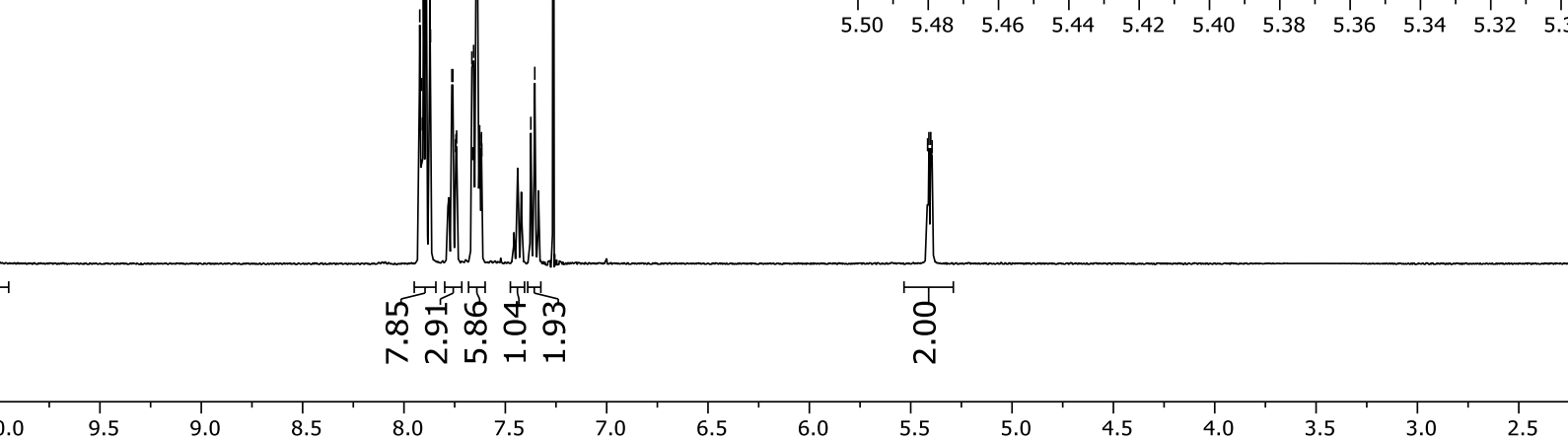

¿্ं

${ }^{1} \mathrm{H}$ NMR spectrum of ( $N$-benzoylamino)methyltriphenylphosphonium bromide (1m); $400 \mathrm{MHz} / \mathrm{CDCl}_{3} / \mathrm{TMS} ; \delta$ (ppm). 


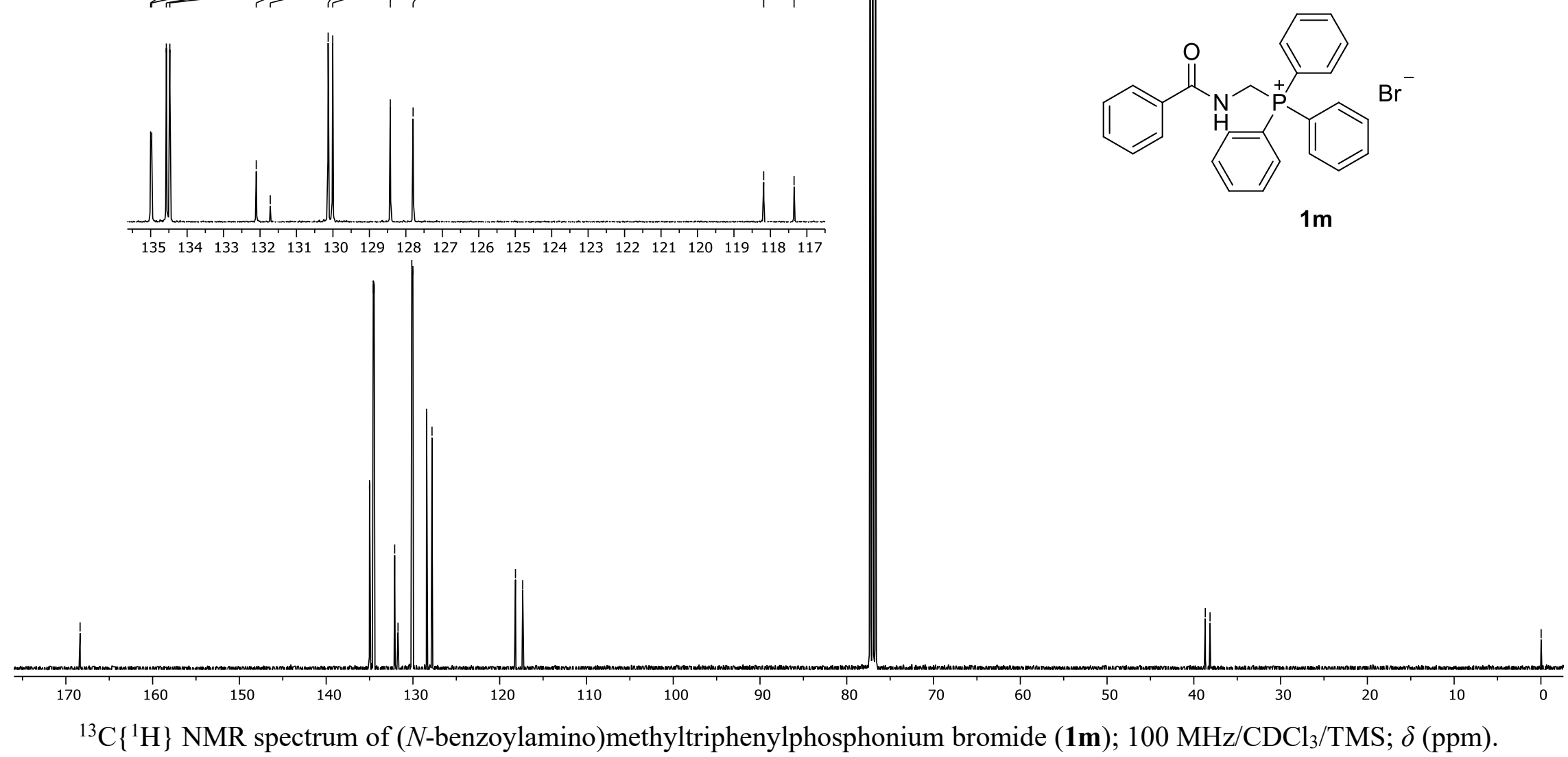




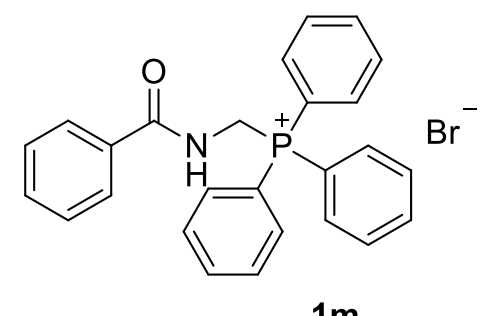

$1 \mathrm{~m}$

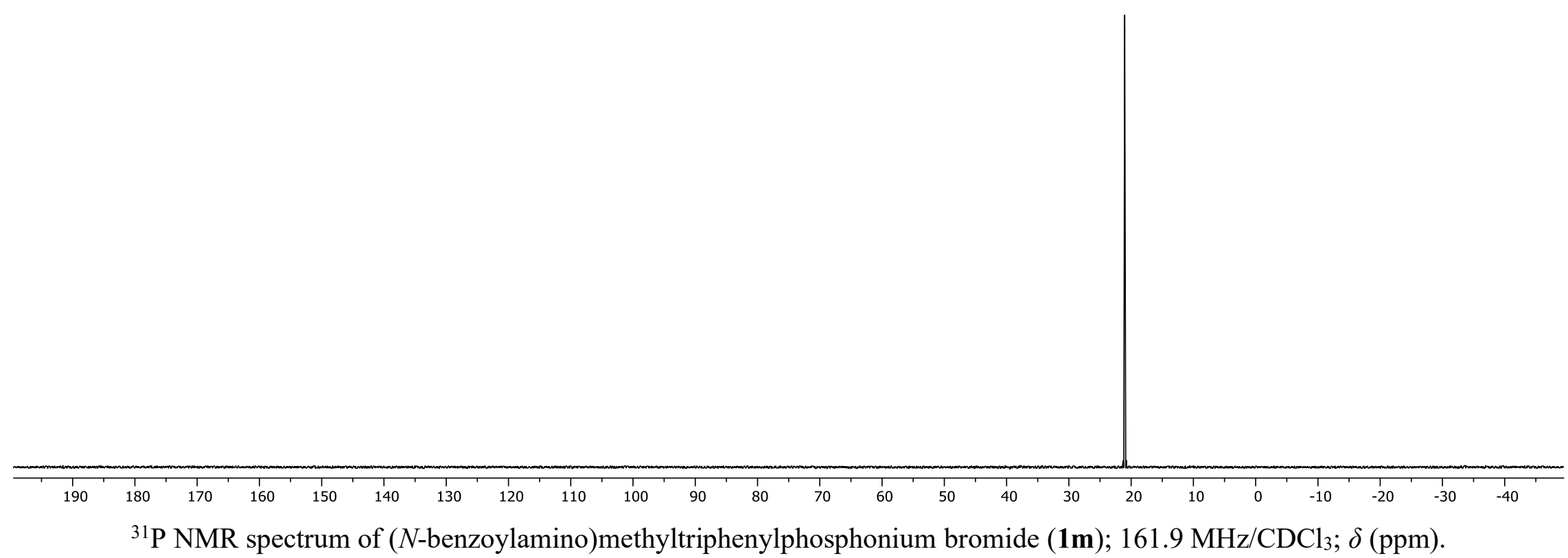




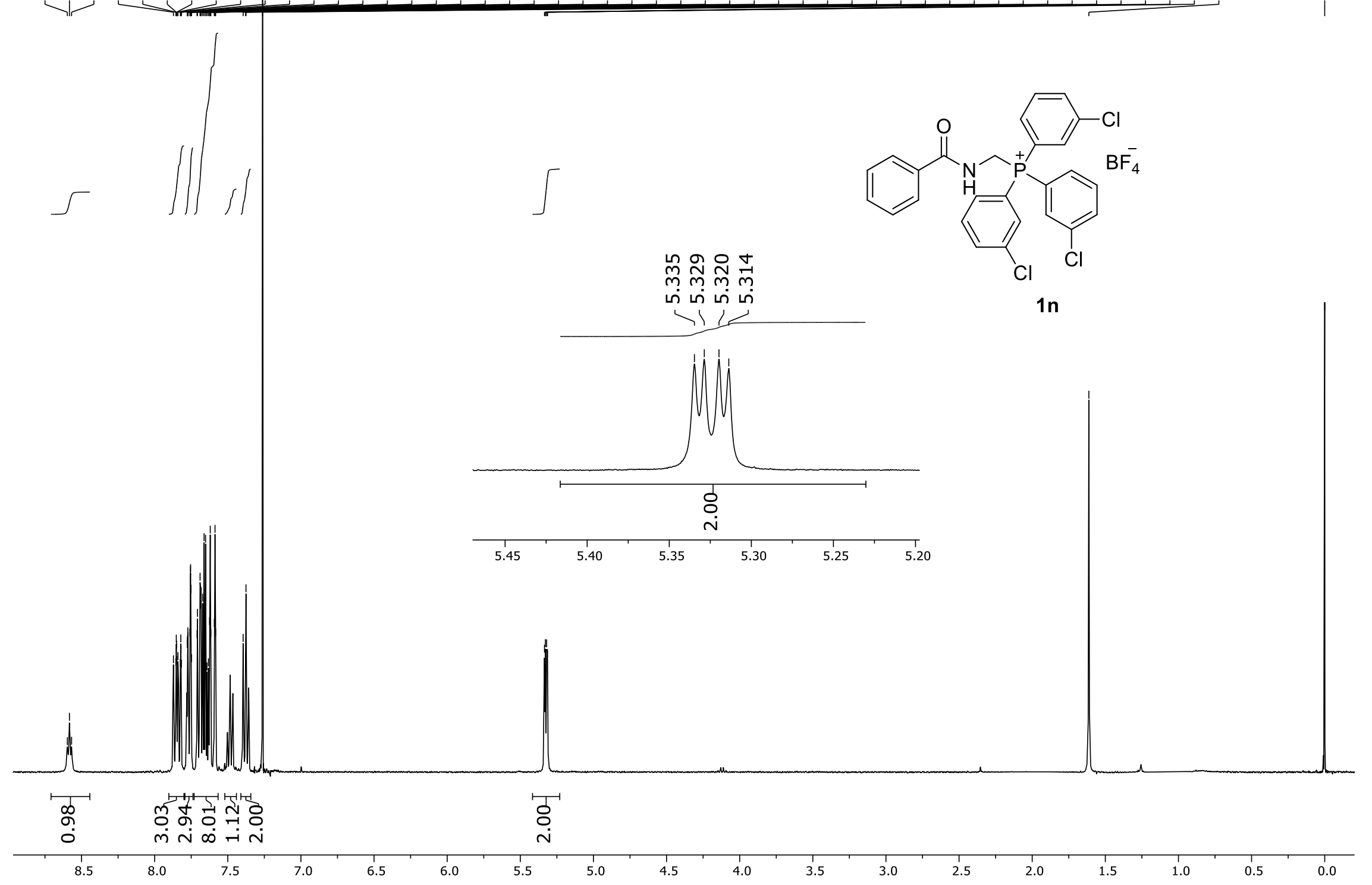

${ }^{1} \mathrm{H}$ NMR spectrum of ( $N$-benzoylamino)methyltris(3-chlorophenyl)phosphonium tetrafluoroborate (1n); $400 \mathrm{MHz} / \mathrm{CDCl}_{3} / \mathrm{TMS} ; \delta$ (ppm). 

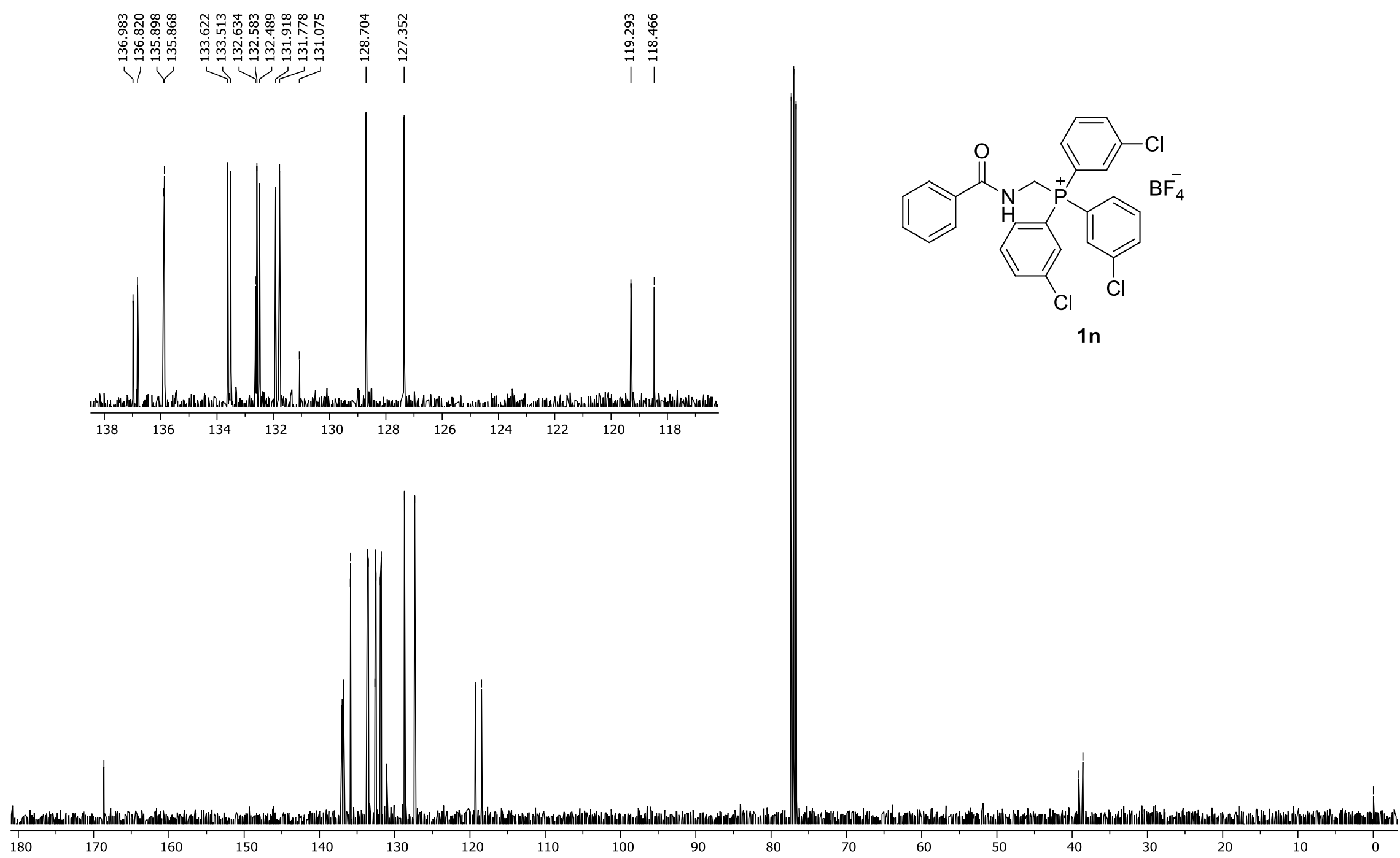

${ }^{13} \mathrm{C}\left\{{ }^{1} \mathrm{H}\right\}$ NMR spectrum of ( $N$-benzoylamino)methyltris(3-chlorophenyl)phosphonium tetrafluoroborate (1n); $100 \mathrm{MHz} / \mathrm{CDCl}_{3} / \mathrm{TMS} ; \delta(\mathrm{ppm})$. 

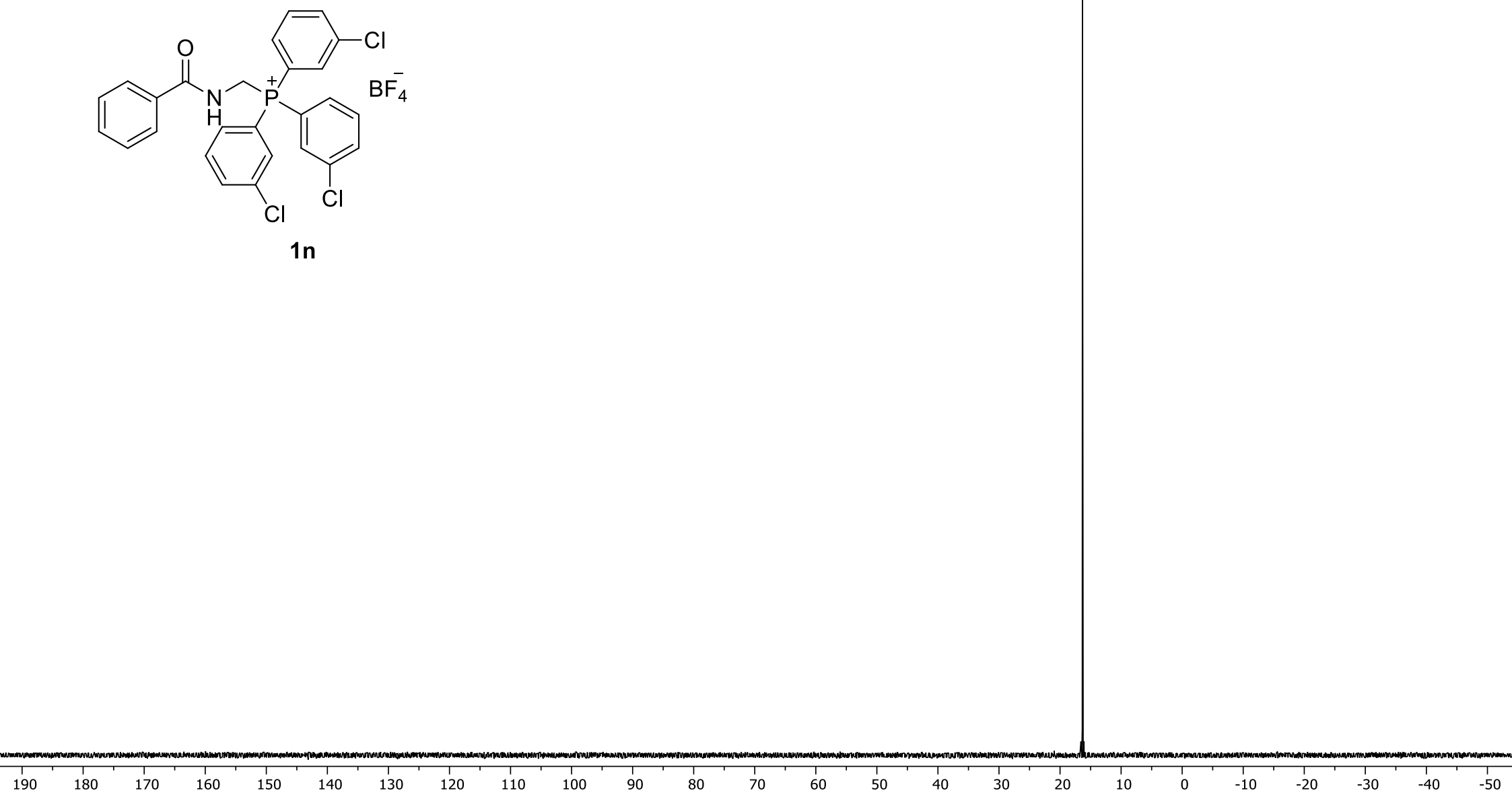

${ }^{31} \mathrm{P}$ NMR spectrum of ( $N$-benzoylamino)methyltris(3-chlorophenyl)phosphonium tetrafluoroborate (1n); $161.9 \mathrm{MHz} / \mathrm{CDCl} ; \delta$ (ppm). 


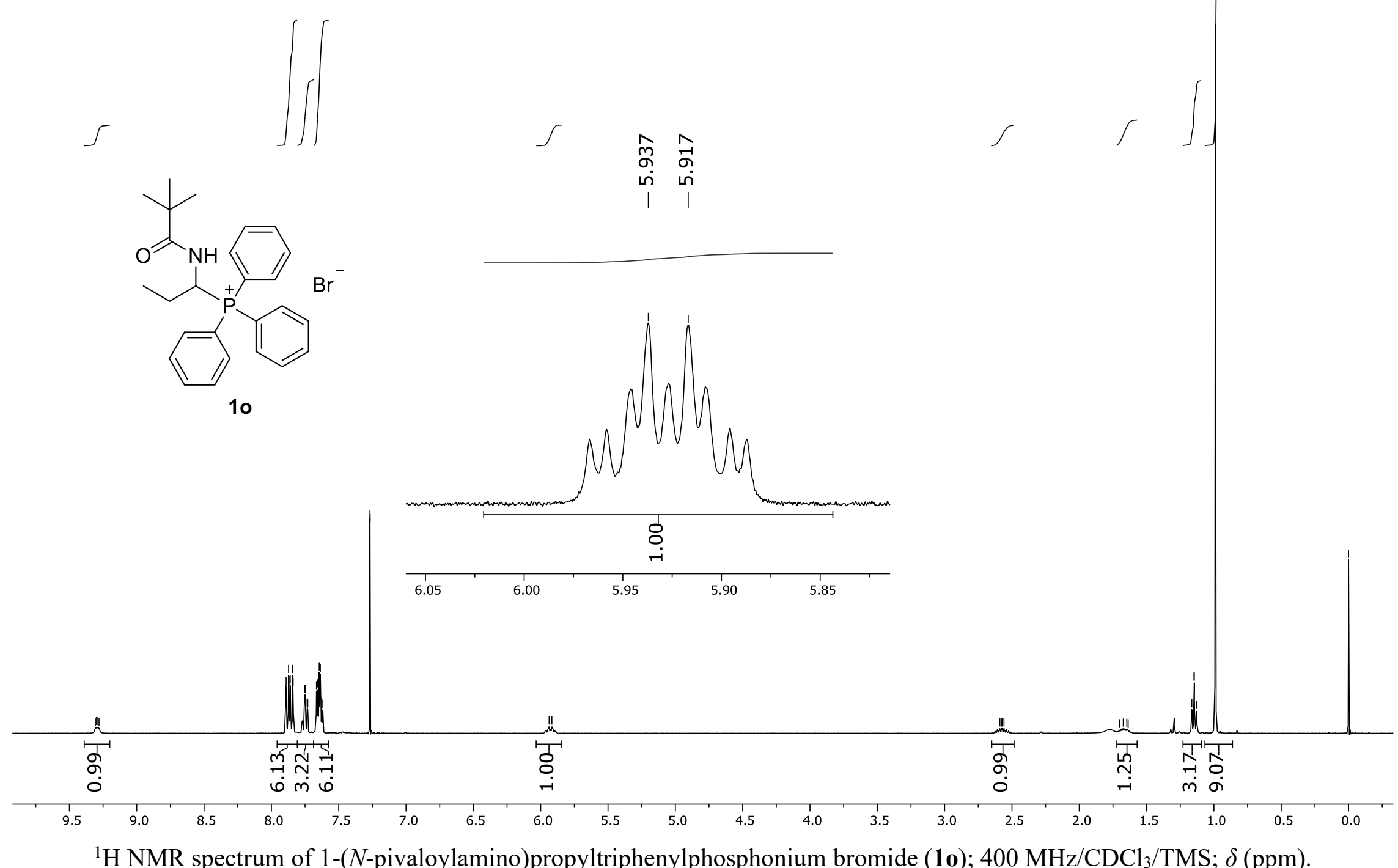

${ }^{1} \mathrm{H}$ NMR spectrum of 1-( $N$-pivaloylamino)propyltriphenylphosphonium bromide (1o); $400 \mathrm{MHz} / \mathrm{CDCl}_{3} / \mathrm{TMS} ; \delta$ (ppm). 


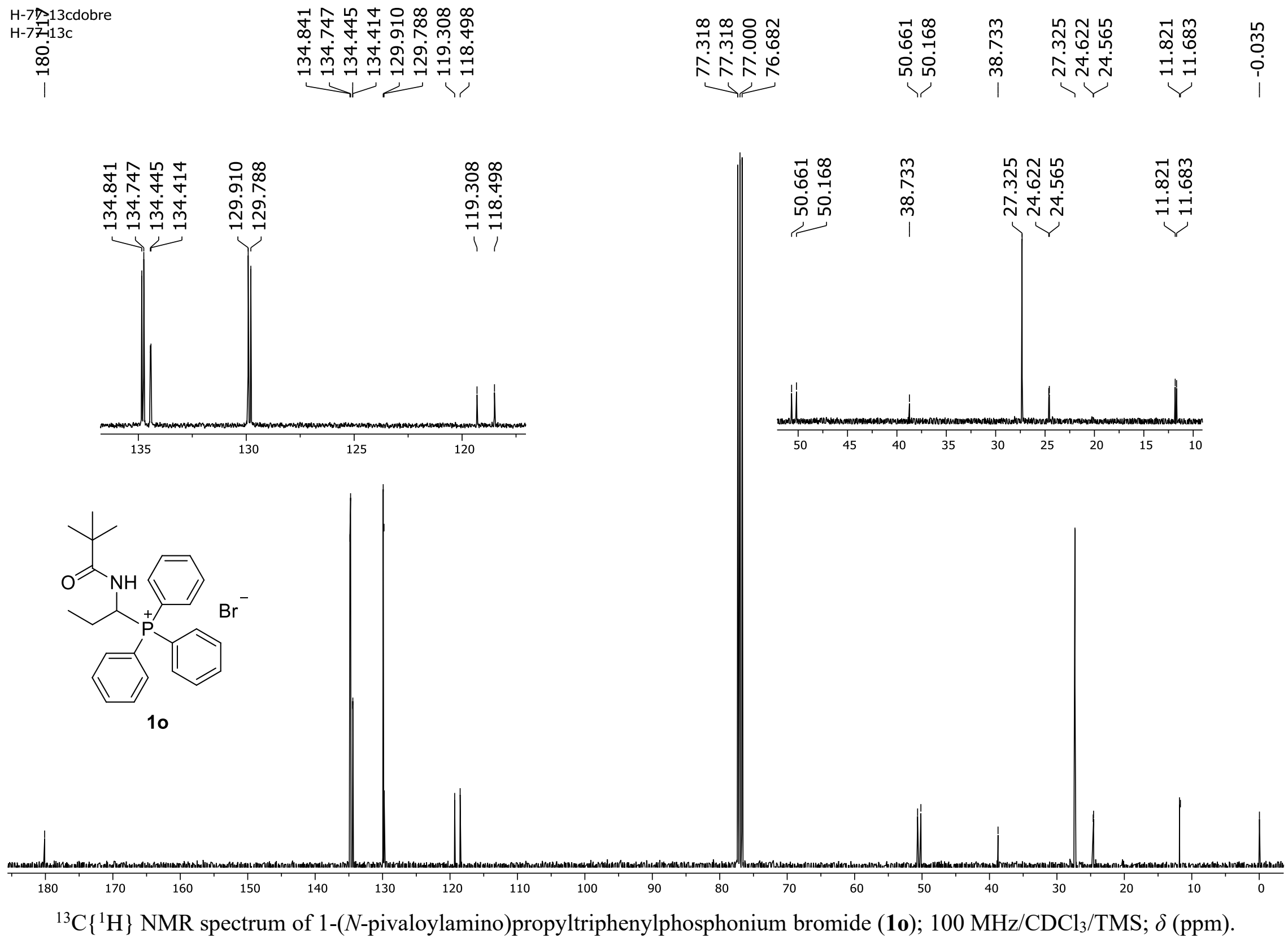




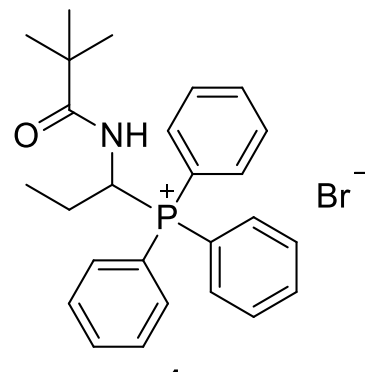

10

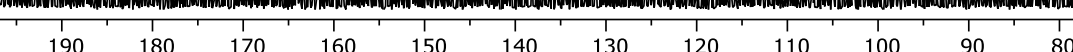

${ }^{31} \mathrm{P}$ NMR spectrum of 1-( $N$-pivaloylamino)propyltriphenylphosphonium bromide (1o); $161.9 \mathrm{MHz} / \mathrm{CDCl}_{3} ; \delta(\mathrm{ppm})$. 


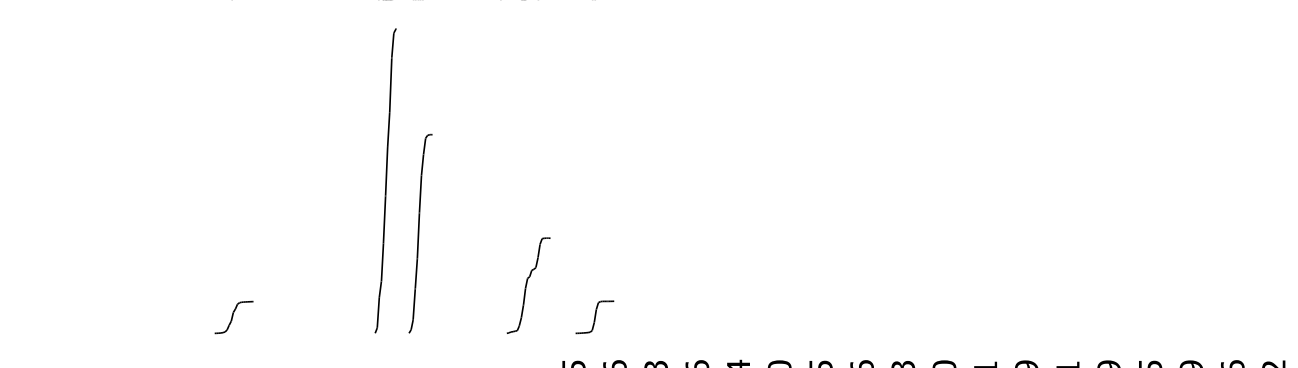

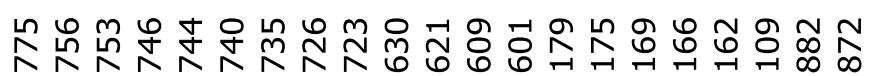

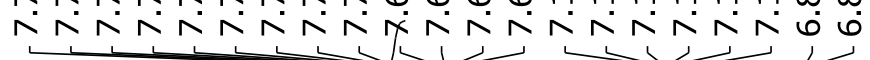
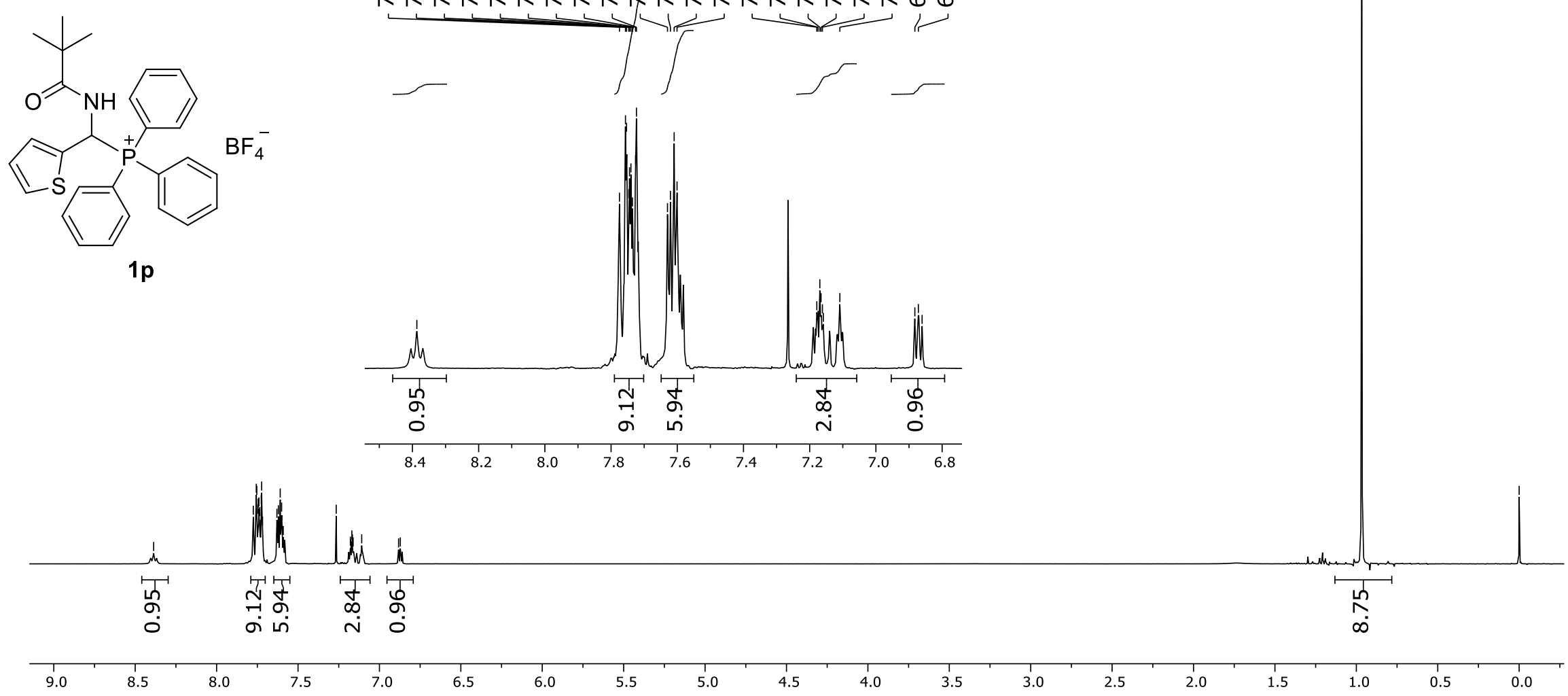

${ }^{1} \mathrm{H}$ NMR spectrum of 1-( $N$-pivaloylamino)-1-(2-thienyl)methyltriphenylphosphonium tetrafluoroborate (1p); $400 \mathrm{MHz} / \mathrm{CDCl}_{3} / \mathrm{TMS} ; \delta$ (ppm). 


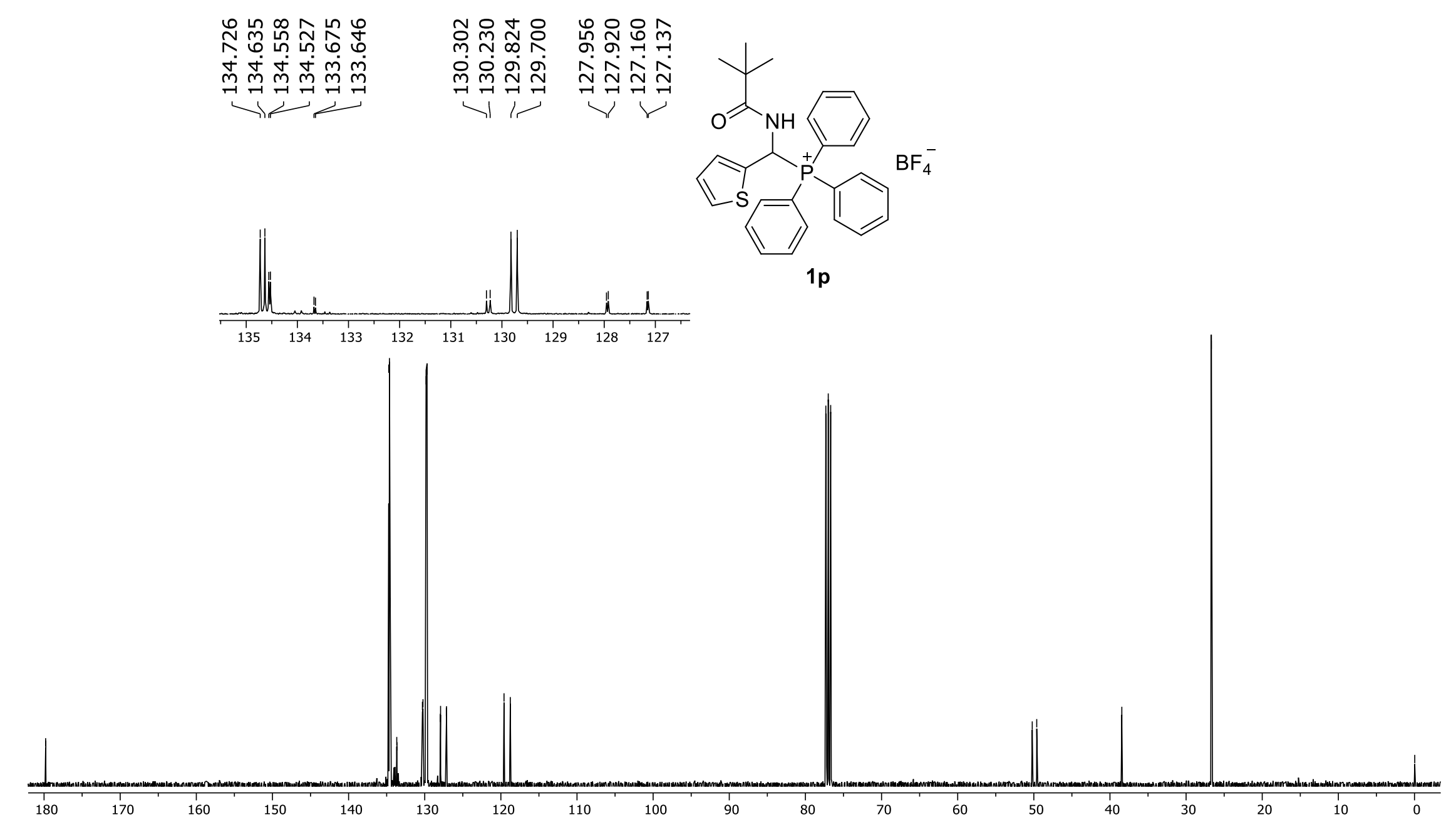

${ }^{13} \mathrm{C}\left\{{ }^{1} \mathrm{H}\right\}$ NMR spectrum of 1-(N-pivaloylamino)-1-(2-thienyl)methyltriphenylphosphonium tetrafluoroborate (1p); $100 \mathrm{MHz} / \mathrm{CDCl} / 3 / \mathrm{TMS} ; \delta$ (ppm). 

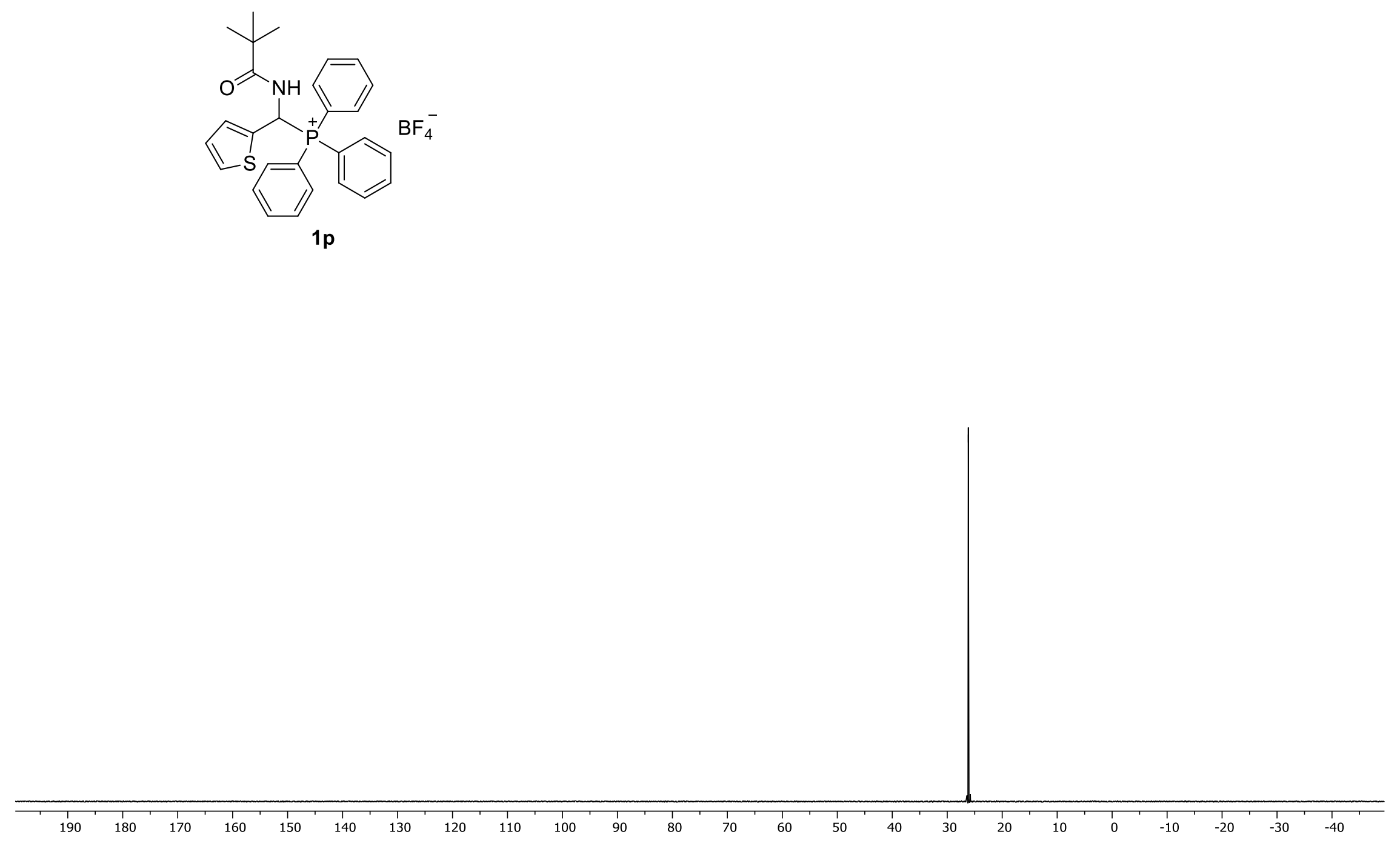

${ }^{31} \mathrm{P}$ NMR spectrum of 1-( $N$-pivaloylamino)-1-(2-thienyl)methyltriphenylphosphonium tetrafluoroborate (1p); $161.9 \mathrm{MHz} / \mathrm{CDCl}_{3} ; \delta(\mathrm{ppm})$. 


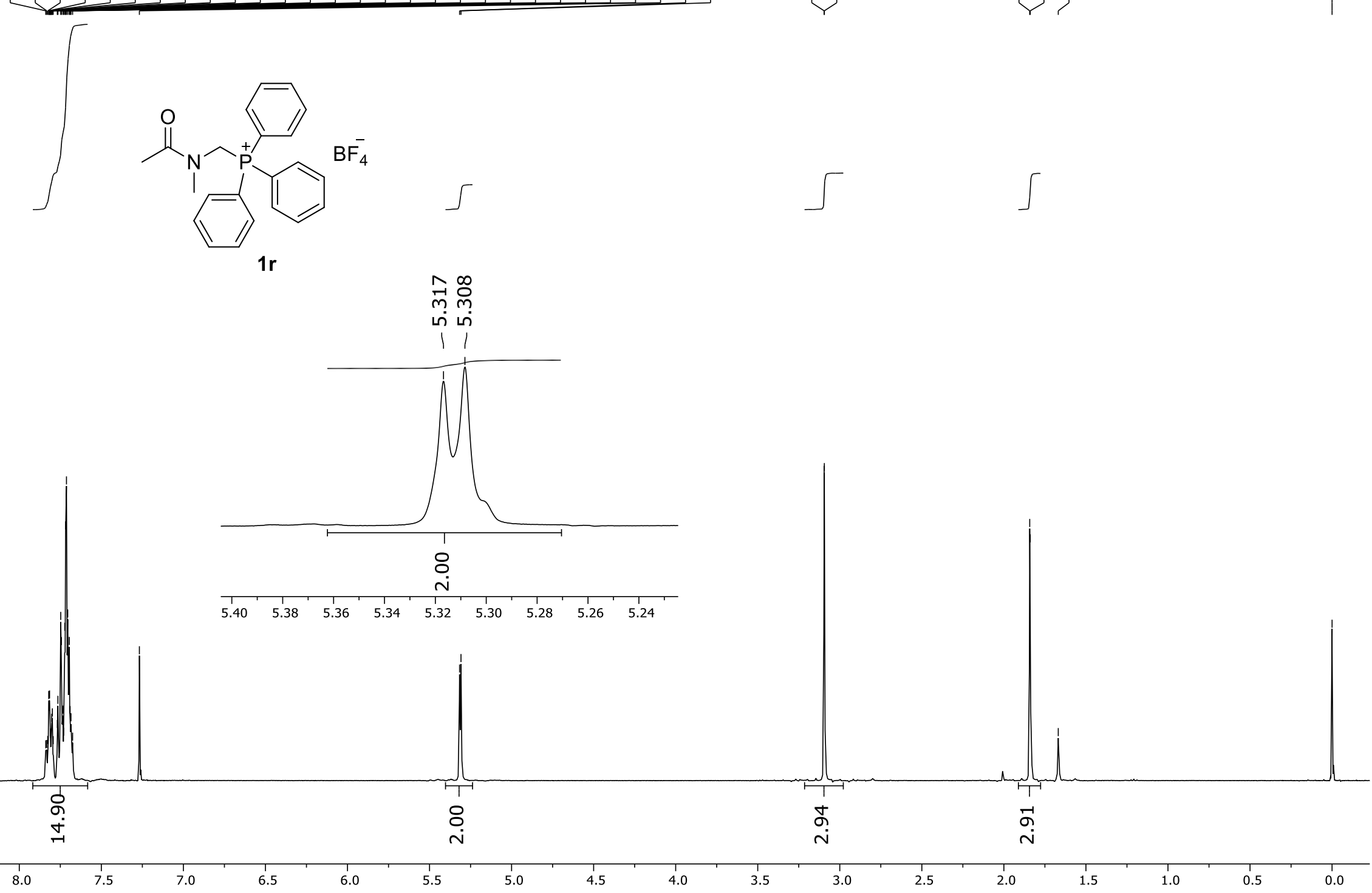

${ }^{1} \mathrm{H}$ NMR spectrum of $N$-( $N$-methylacetylamino)methyltriphenylphosphonium tetrafluoroborate (1r); $400 \mathrm{MHz} / \mathrm{CDCl} 3 / \mathrm{TMS} ; \delta$ (ppm). 


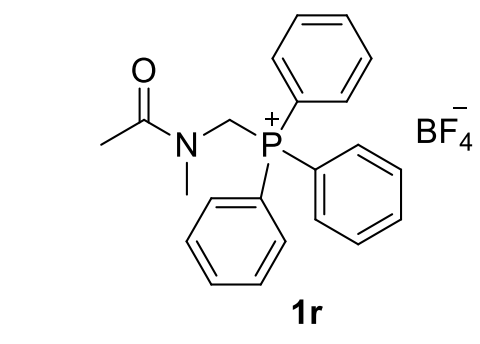

$\mathrm{BF}_{4}^{-}$

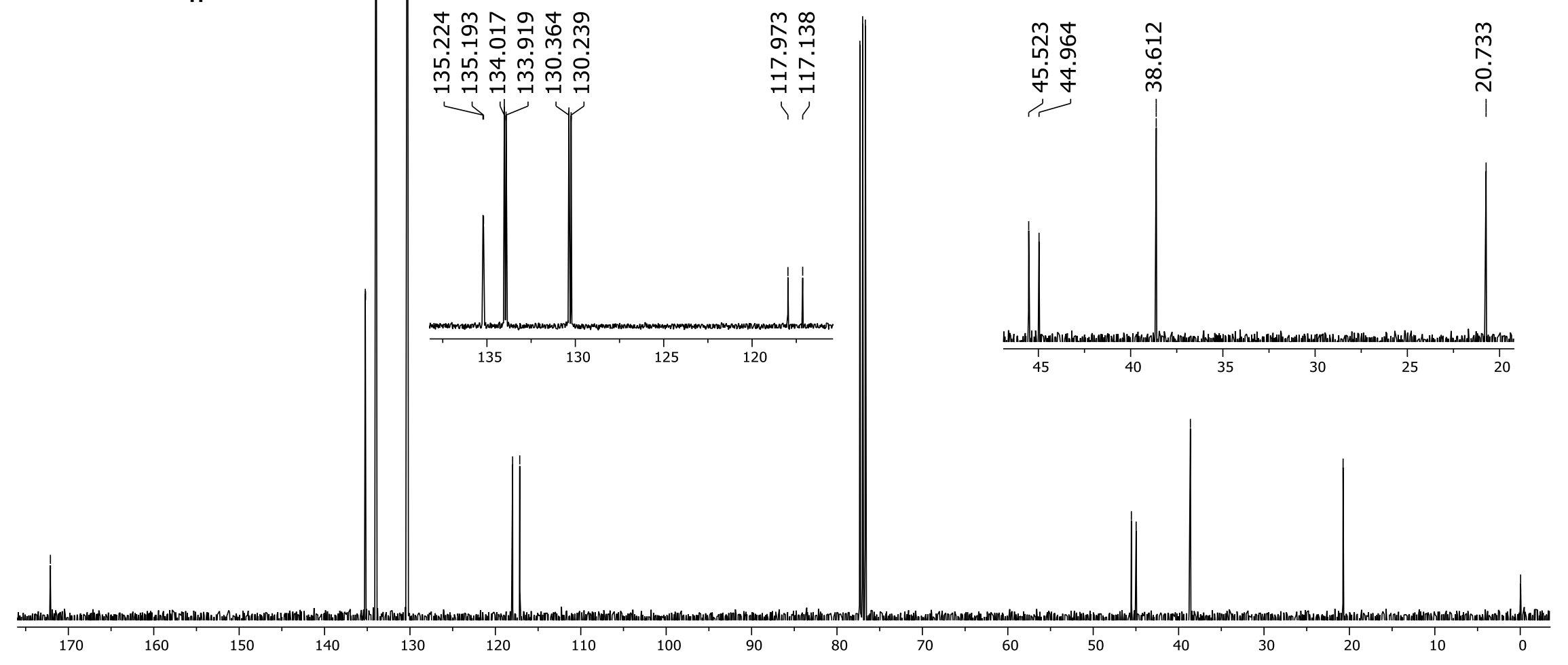

${ }^{13} \mathrm{C}\left\{{ }^{1} \mathrm{H}\right\}$ NMR spectrum of $N$-(N-methylacetylamino)methyltriphenylphosphonium tetrafluoroborate (1r); $100 \mathrm{MHz} / \mathrm{CDCl}_{3} / \mathrm{TMS} ; \delta(\mathrm{ppm})$. 

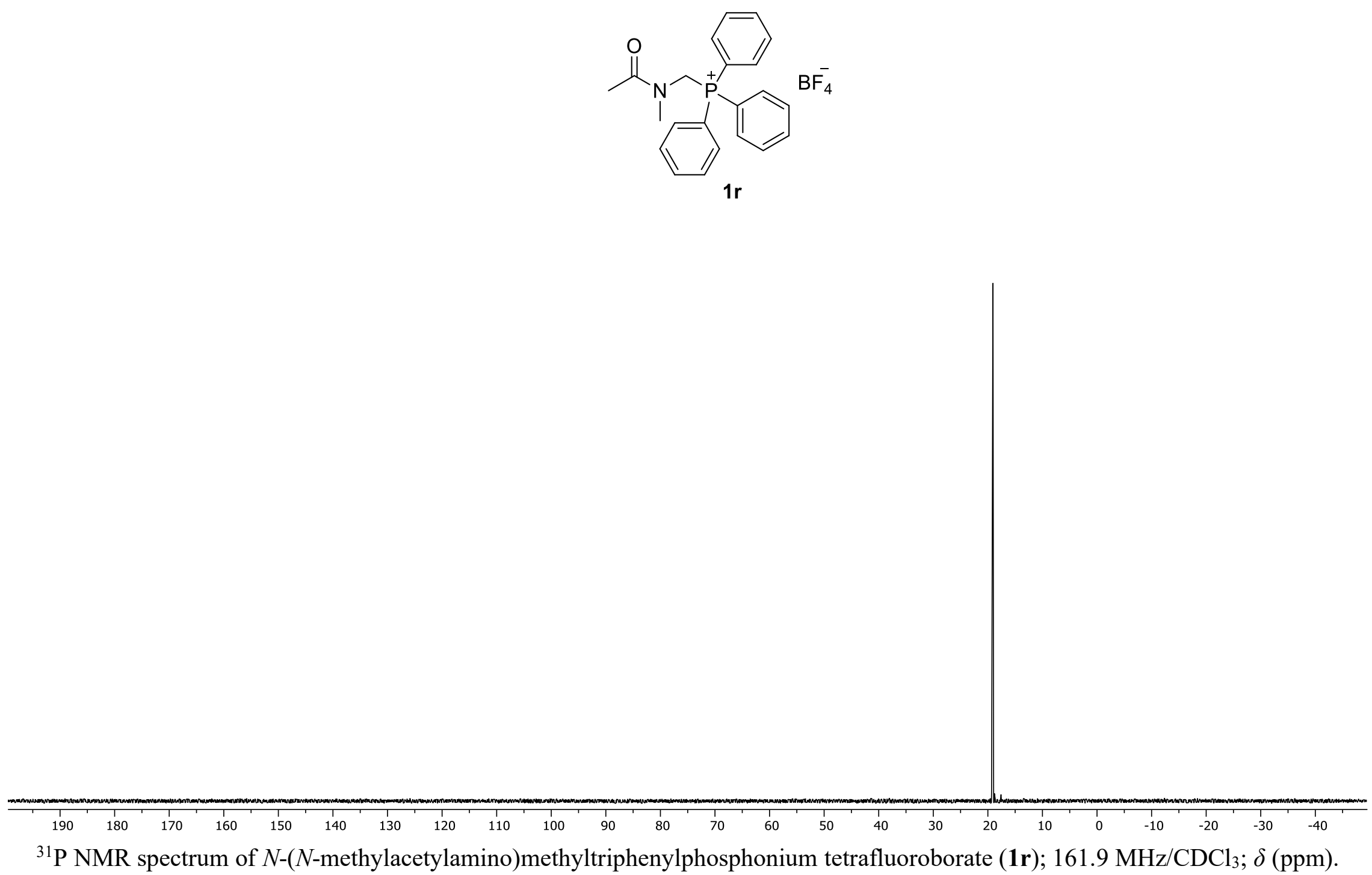

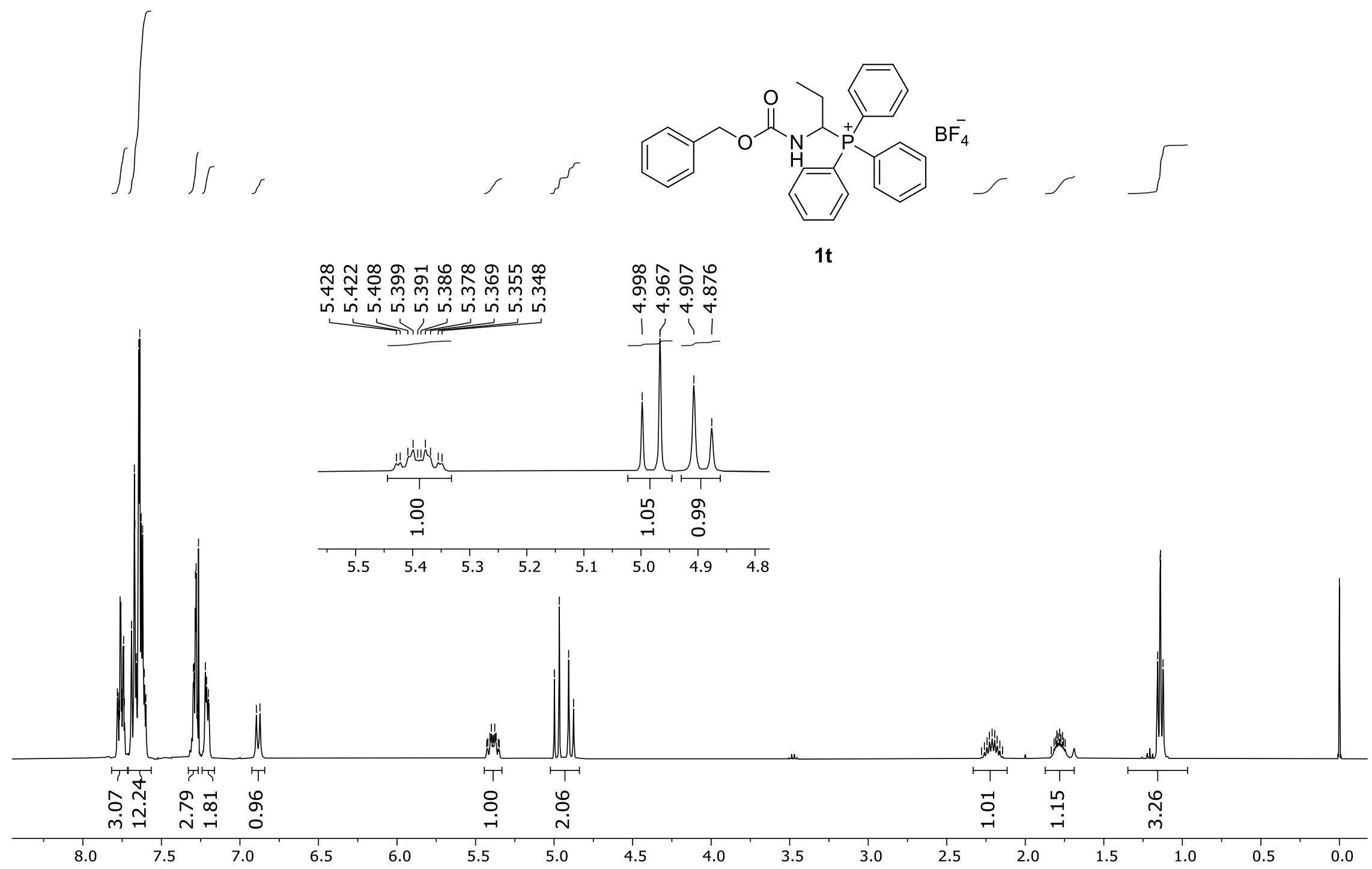

${ }^{1} \mathrm{H}$ NMR spectrum of 1 -( $N$-benzyloxycarbonylamino)propyltriphenylphosphonium tetrafluoroborate (1t); $400 \mathrm{MHz} / \mathrm{CDCl} / \mathrm{TMS} ; \delta$ (ppm). 


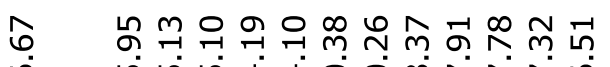

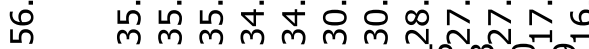

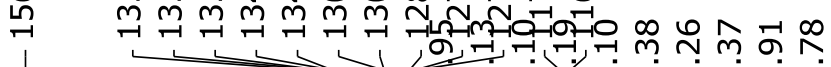

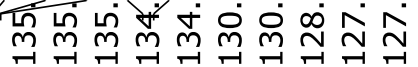

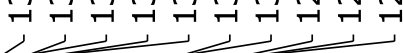

$\frac{m}{\mathrm{y}} \frac{m}{\mathrm{i}} \frac{m}{\mathrm{v}}$

mo n m. 큭

$>$

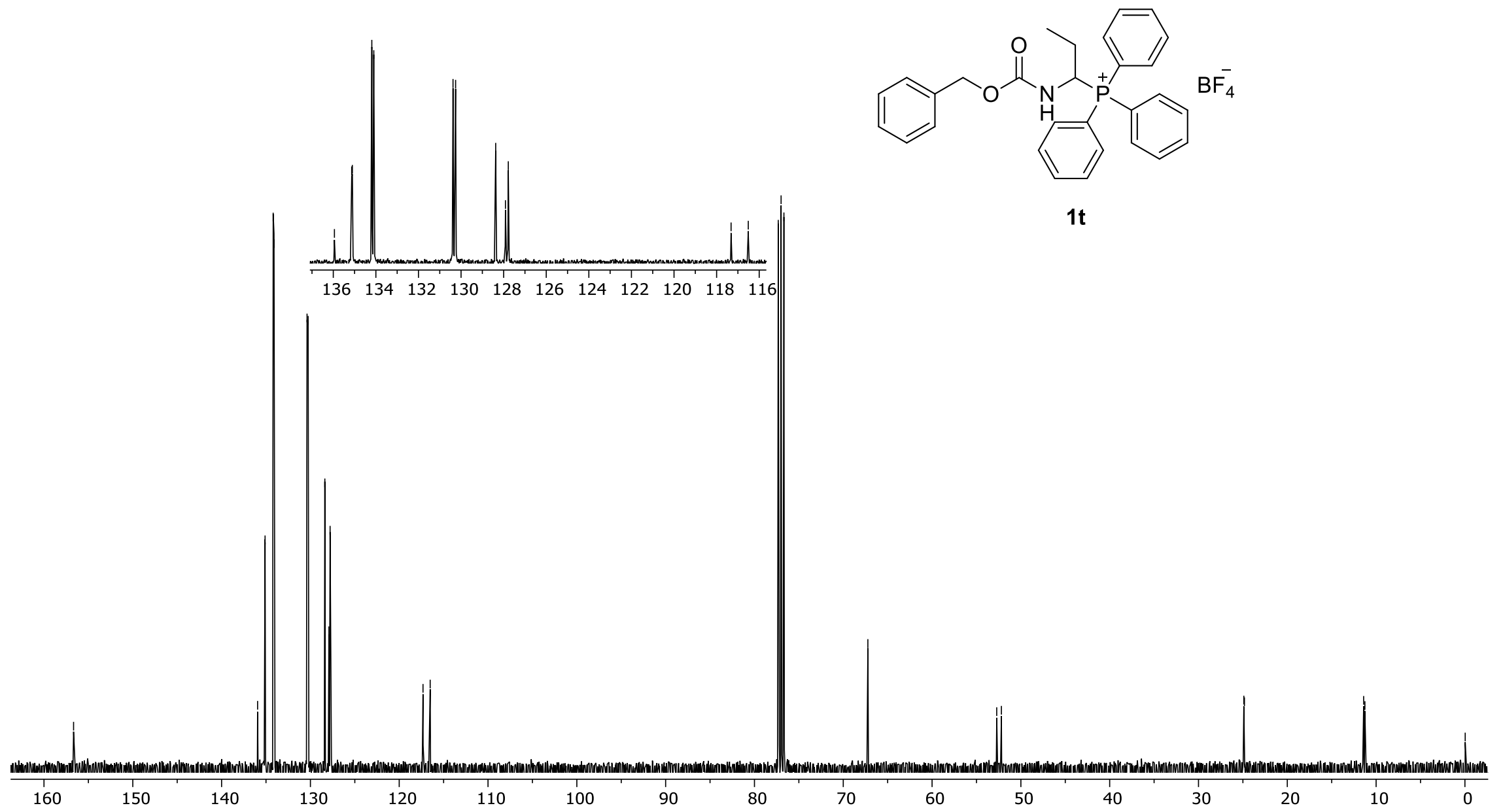

${ }^{13} \mathrm{C}\left\{{ }^{1} \mathrm{H}\right\}$ NMR spectrum of 1-( $N$-benzyloxycarbonylamino)propyltriphenylphosphonium tetrafluoroborate (1t); $100 \mathrm{MHz} / \mathrm{CDCl}_{3} / \mathrm{TMS} ; \delta(\mathrm{ppm})$. 


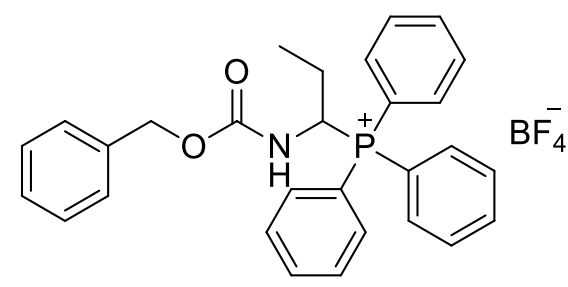

1t

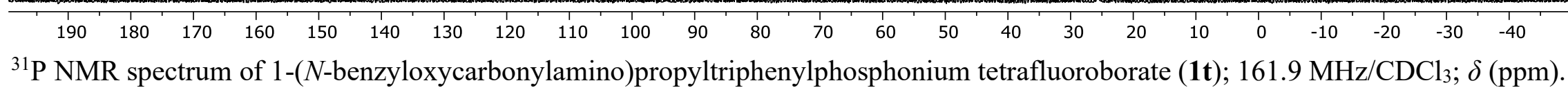



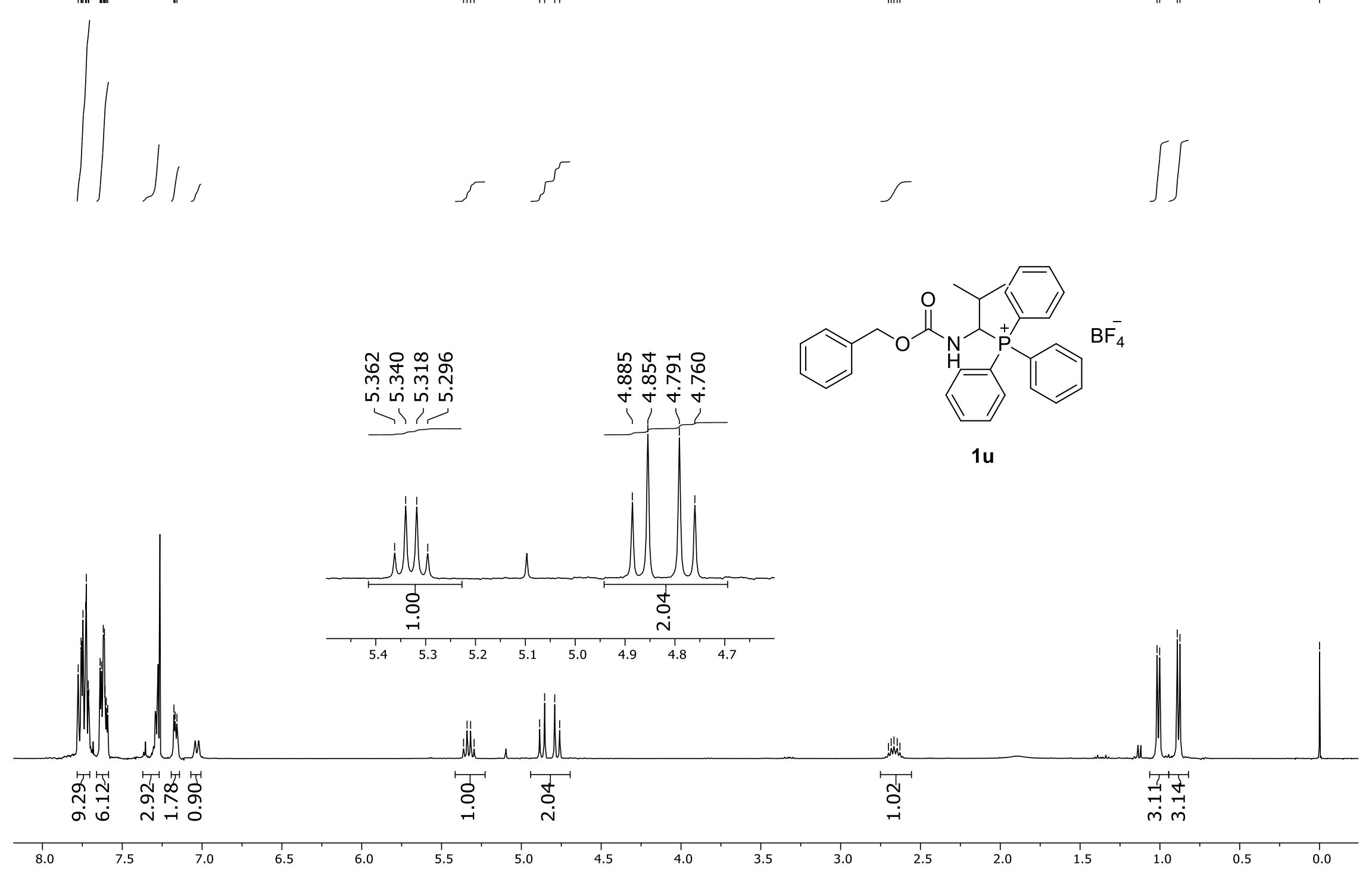

${ }^{1} \mathrm{H}$ NMR spectrum of 1-( $N$-benzyloxycarbonylamino)-2-methylpropyltriphenylphosphonium tetrafluoroborate (1u); $400 \mathrm{MHz} / \mathrm{CDCl} 3 / \mathrm{TMS} ; \delta$ (ppm). 

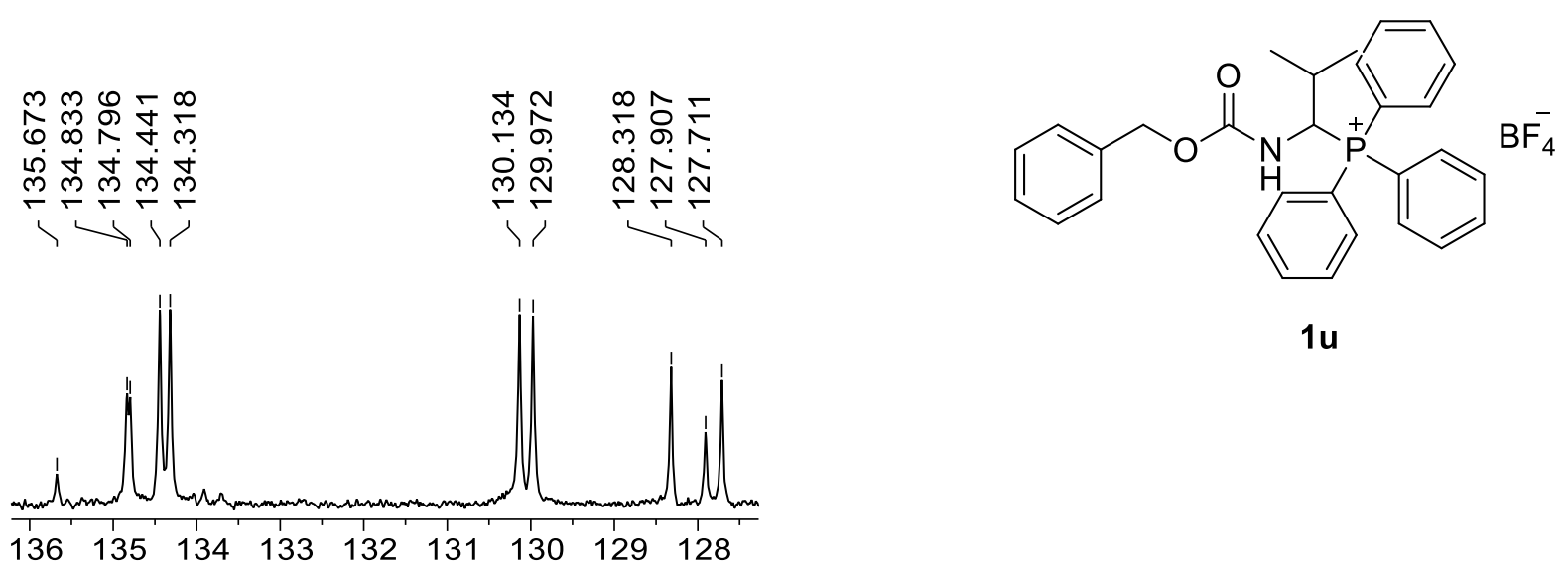

1u

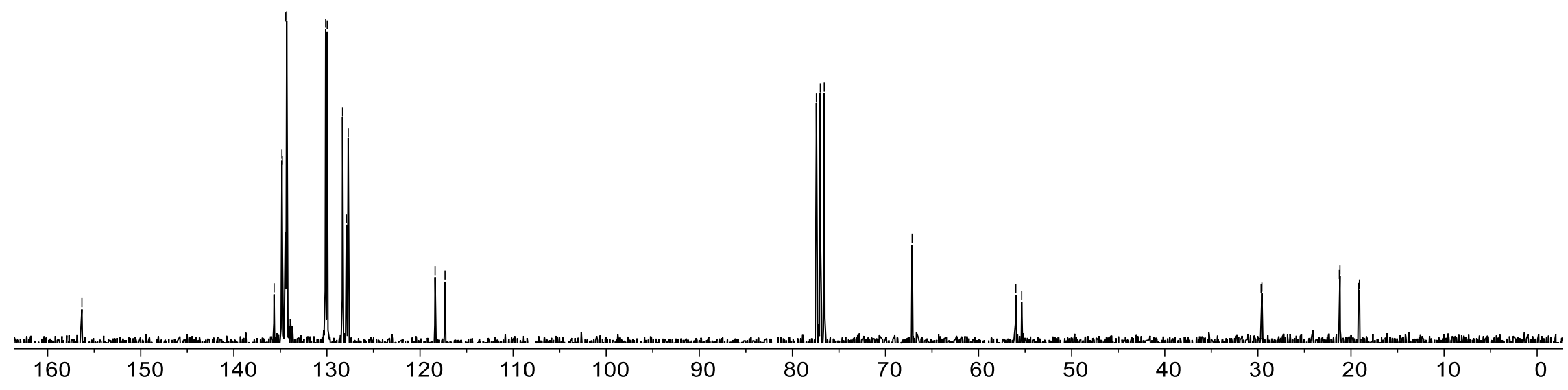

${ }^{13} \mathrm{C}\left\{{ }^{1} \mathrm{H}\right\}$ NMR spectrum of 1-( $N$-benzyloxycarbonylamino)-2-methylpropyltriphenylphosphonium tetrafluoroborate $(1 \mathbf{u}) ; 100 \mathrm{MHz} / \mathrm{CDCl} / \mathrm{TMS} ; \delta$ (ppm). 


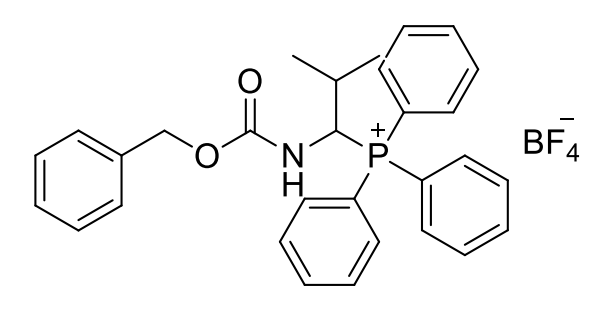

1u

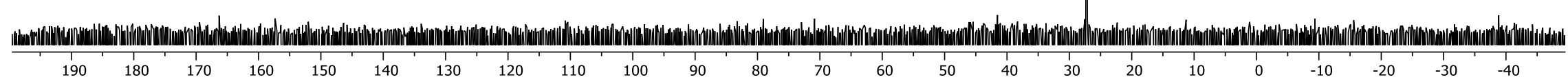

${ }^{31} \mathrm{P}$ NMR spectrum of 1-( $N$-benzyloxycarbonylamino)-2-methylpropyltriphenylphosphonium tetrafluoroborate $(1 \mathbf{u}) ; 161.9 \mathrm{MHz} / \mathrm{CDCl}_{3} ; \delta$ (ppm). 


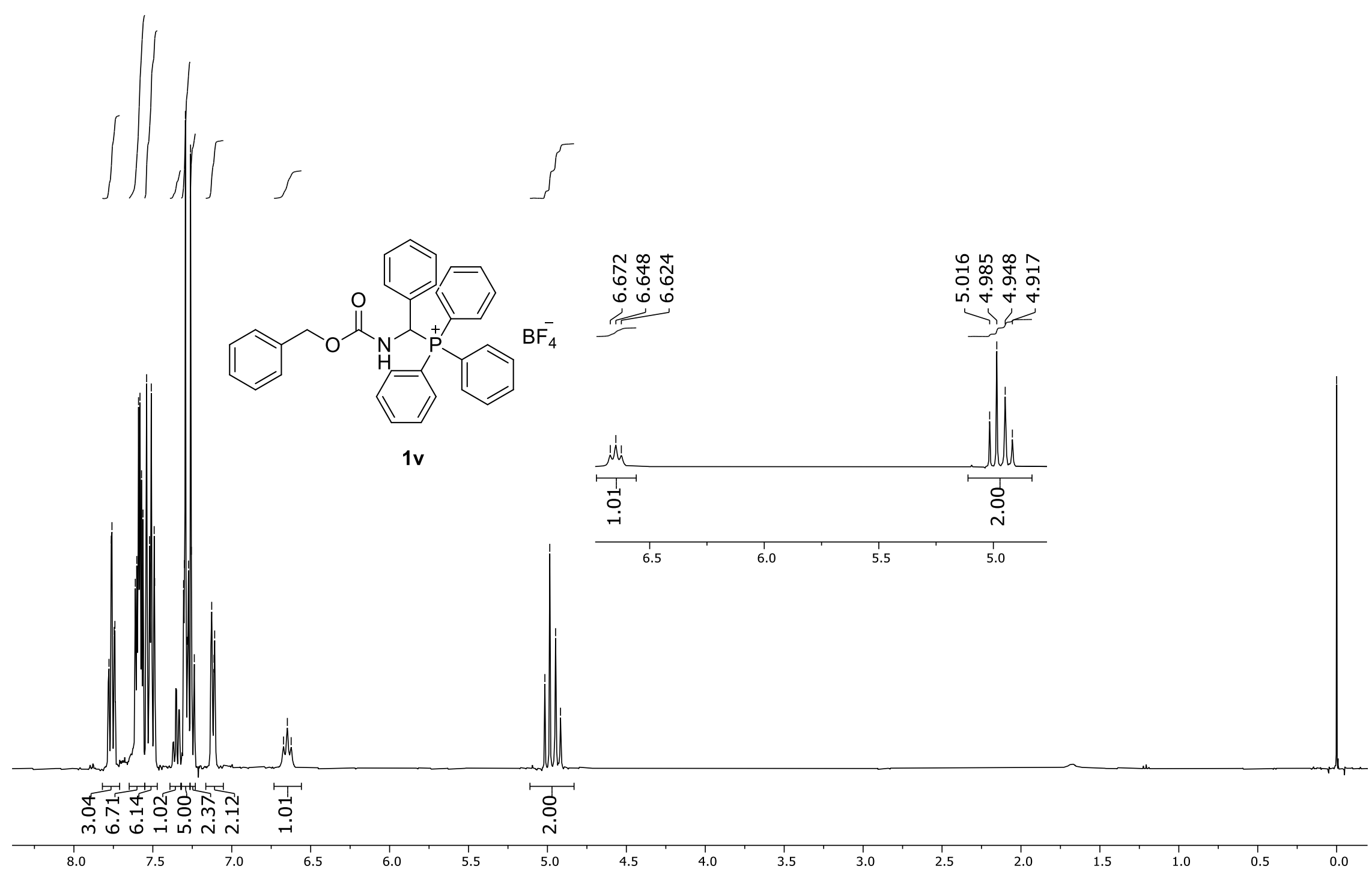

${ }^{1} \mathrm{H}$ NMR spectrum of ( $N$-benzyloxycarbonylamino)phenylmethyltriphenylphosphonium tetrafluoroborate (1v); $400 \mathrm{MHz} / \mathrm{CDCl} / \mathrm{TMS} ; \delta$ (ppm). 


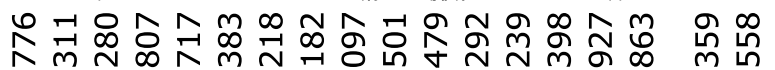

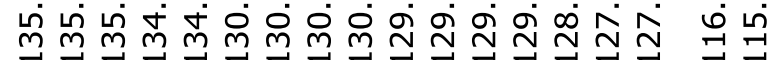
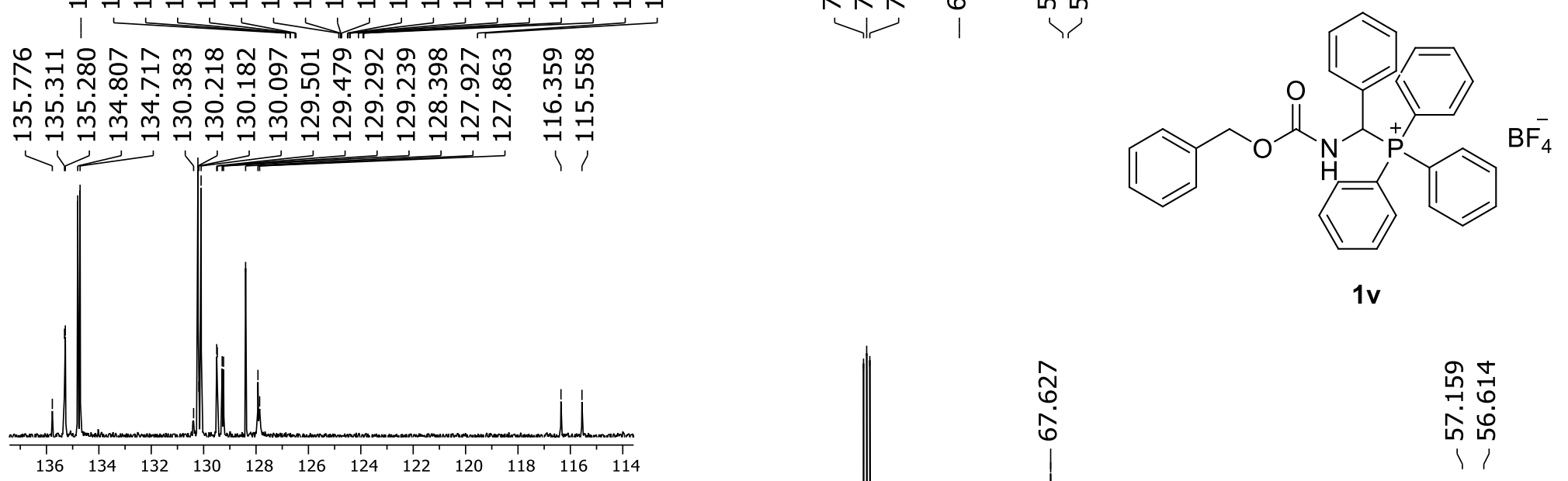

1v

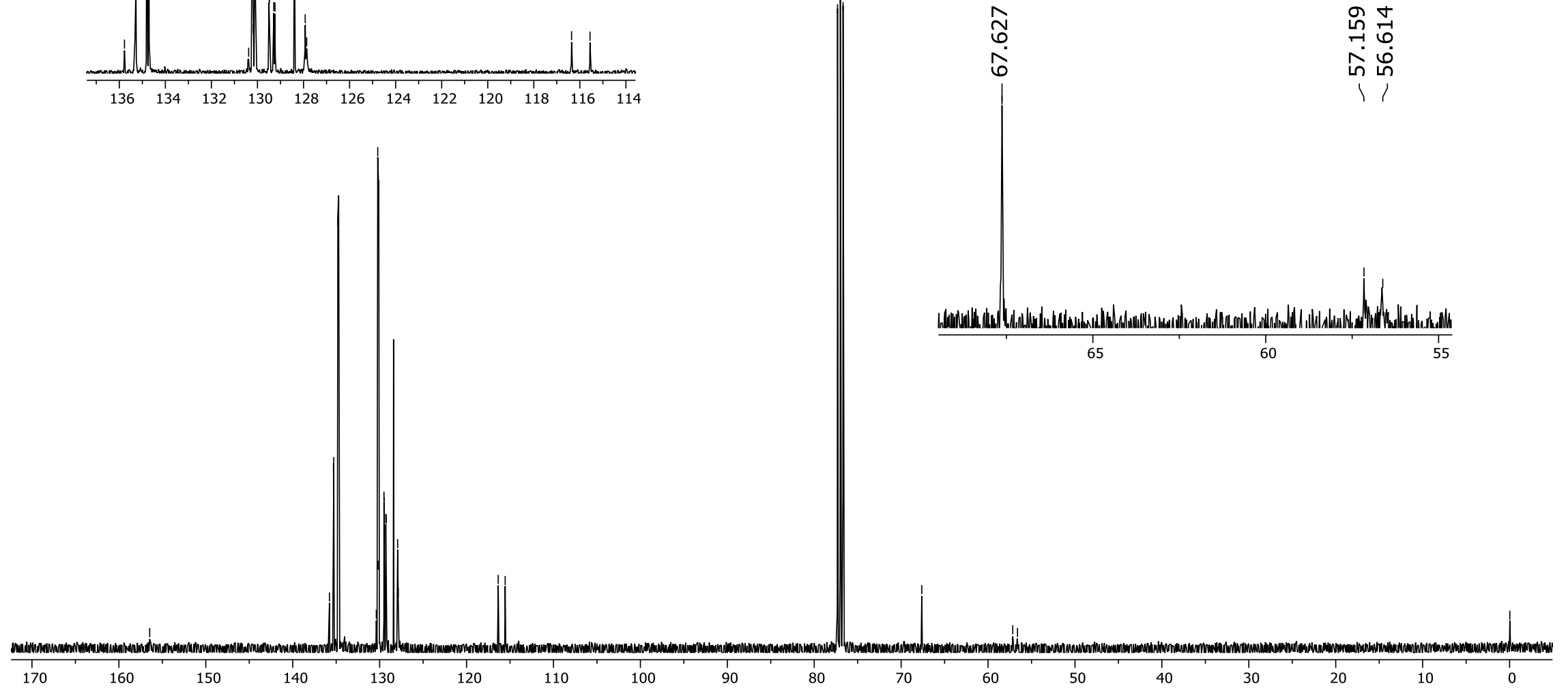

${ }^{13} \mathrm{C}\left\{{ }^{1} \mathrm{H}\right\}$ NMR spectrum of ( $N$-benzyloxycarbonylamino)phenylmethyltriphenylphosphonium tetrafluoroborate $(1 \mathbf{v}) ; 100 \mathrm{MHz} / \mathrm{CDCl} / \mathrm{TMS} ; \delta$ (ppm). 


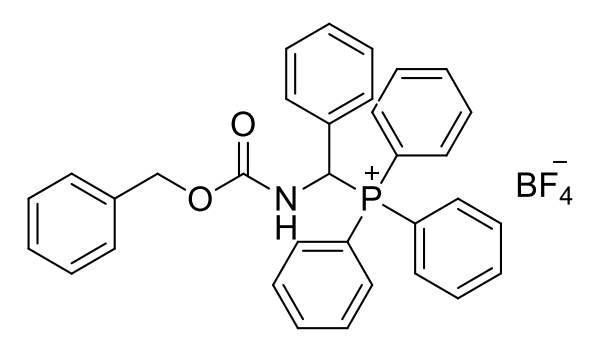

1v

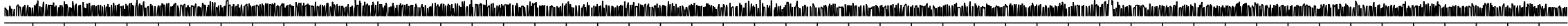

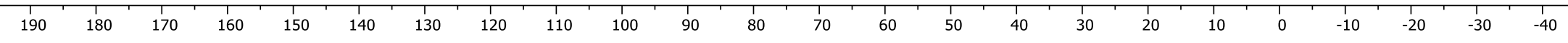

${ }^{31} \mathrm{P}$ NMR spectrum of ( $N$-benzyloxycarbonylamino)phenylmethyltriphenylphosphonium tetrafluoroborate $(\mathbf{1 v}) ; 161.9 \mathrm{MHz} / \mathrm{CDCl}_{3} ; \delta$ (ppm). 


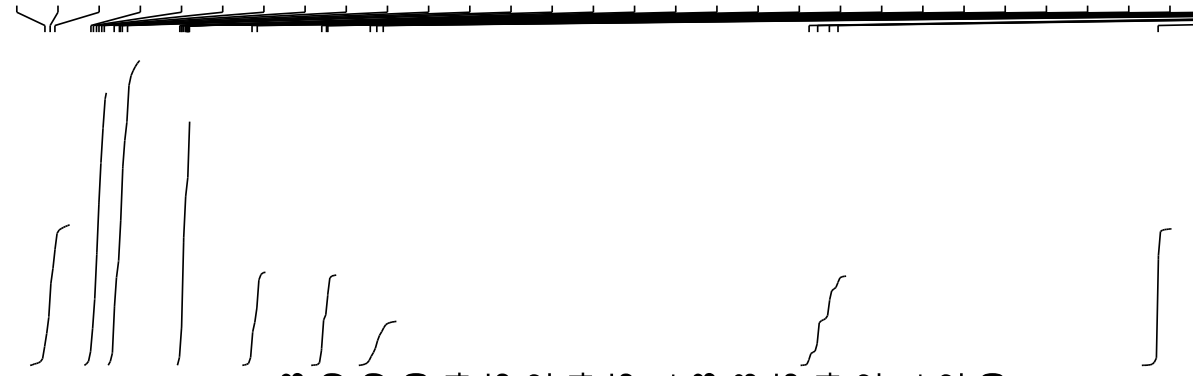

央 ᄋำ
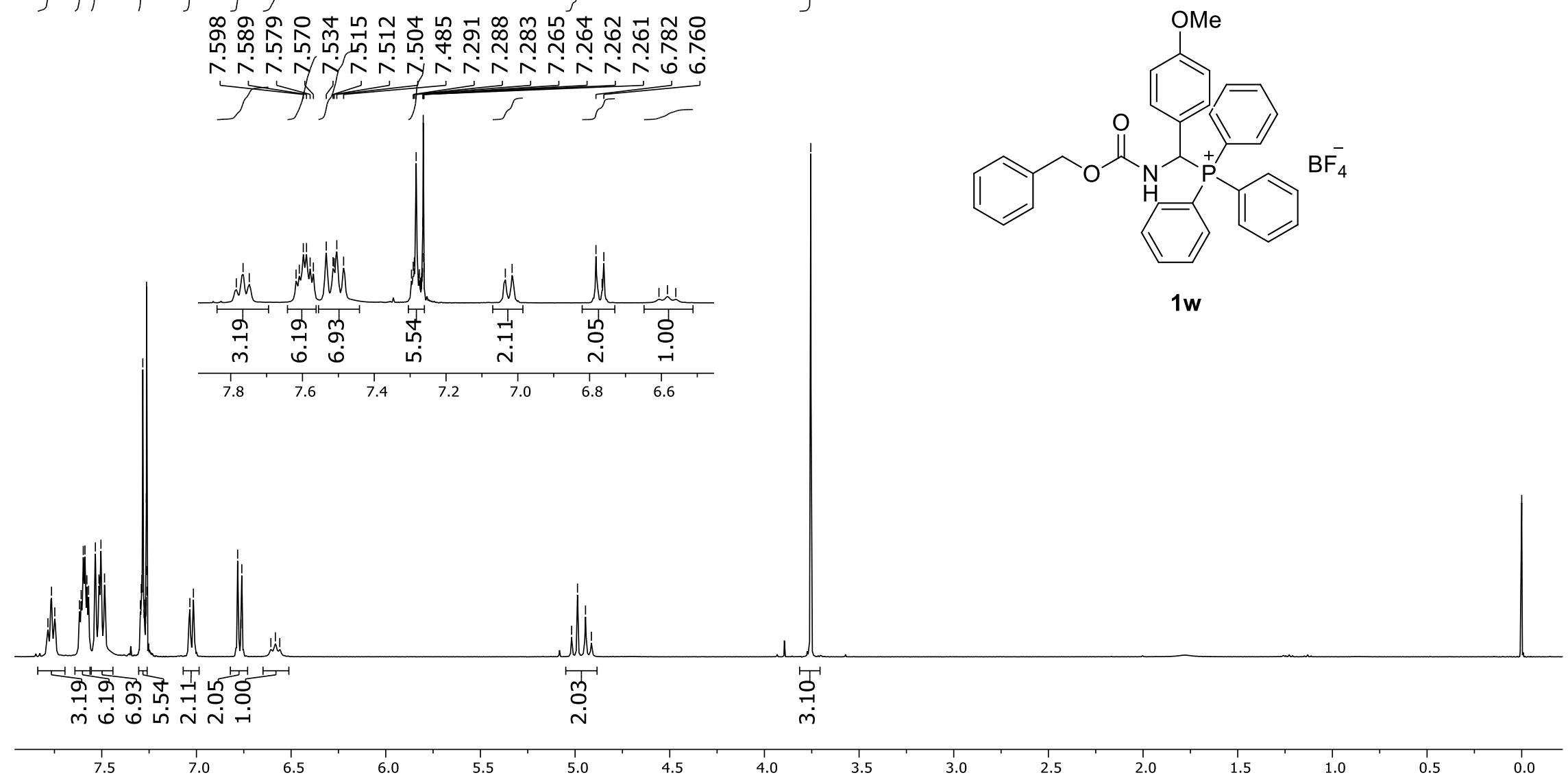

$1 \mathrm{w}$

${ }^{1} \mathrm{H}$ NMR spectrum of 1-( $N$-benzyloxycarbonylamino)-1-(4-methoxyphenyl)methyltriphenylphosphonium tetrafluoroborate (1 w); $400 \mathrm{MHz} / \mathrm{CDCl} / \mathrm{TMS}_{3} \delta$ (ppm). 


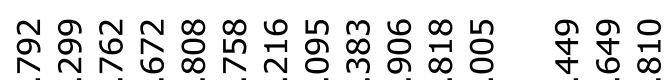

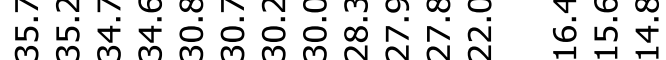

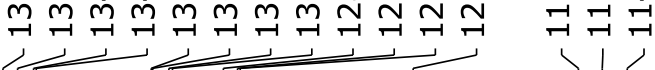
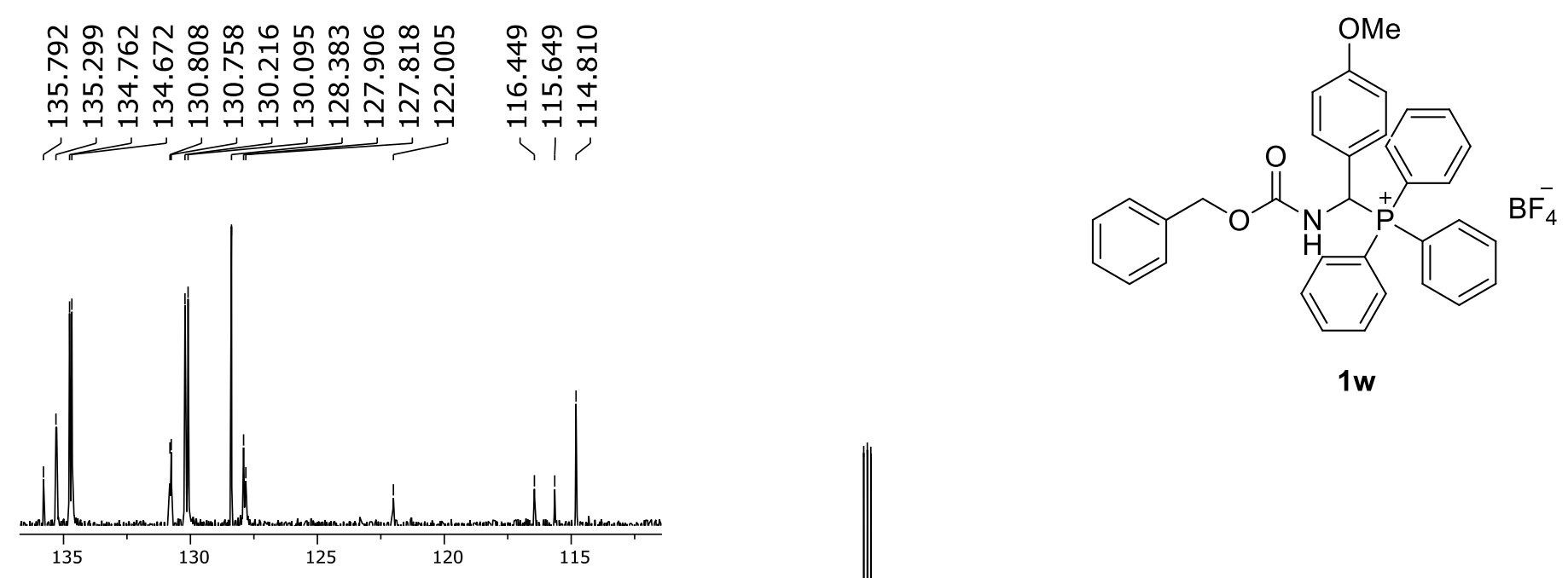

$1 w$

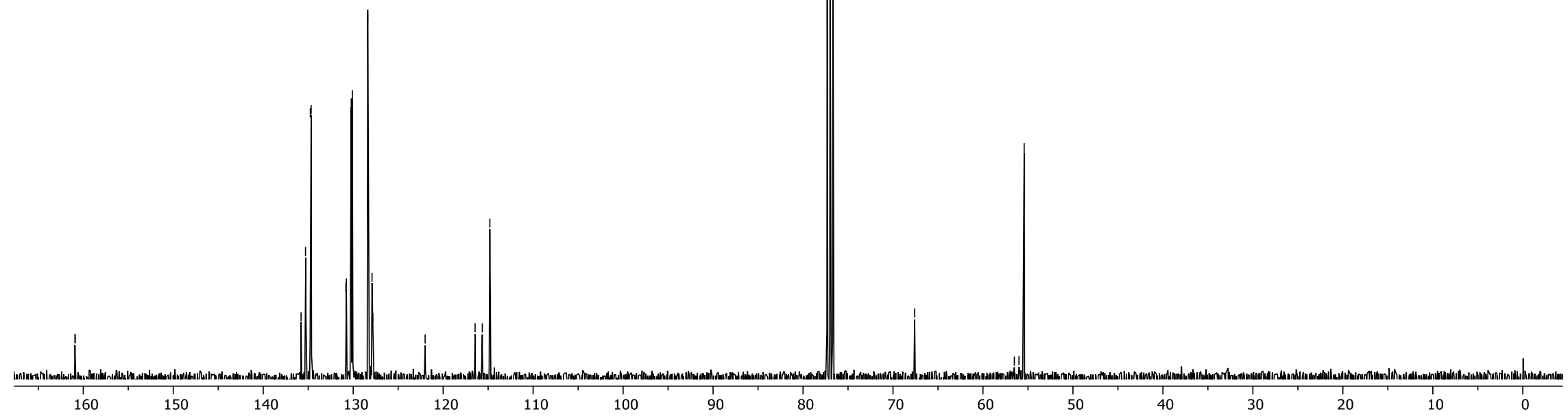

${ }^{13} \mathrm{C}\left\{{ }^{1} \mathrm{H}\right\}$ NMR spectrum of 1-(N-benzyloxycarbonylamino)-1-(4-methoxyphenyl)methyltriphenylphosphonium tetrafluoroborate (1w); $100 \mathrm{MHz} / \mathrm{CDCl} / 3 / \mathrm{TMS} ; \delta$ (ppm). 


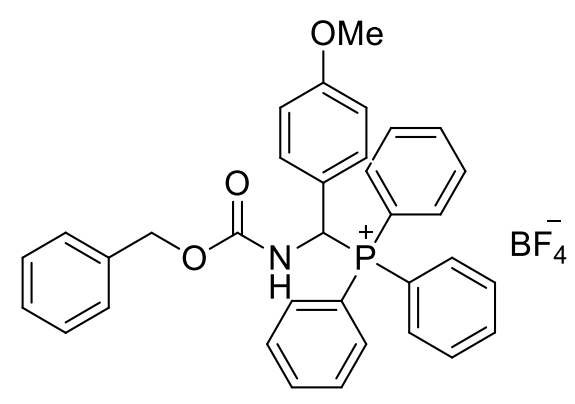

$1 w$

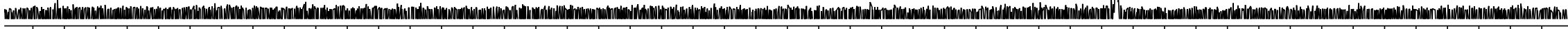

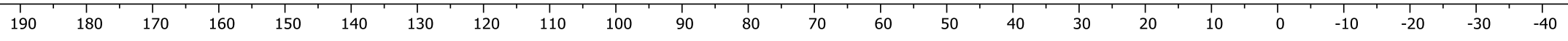

${ }^{31} \mathrm{P}$ NMR spectrum of 1-(N-benzyloxycarbonylamino)-1-(4-methoxyphenyl)methyltriphenylphosphonium tetrafluoroborate (1w); $161.9 \mathrm{MHz} / \mathrm{CDCl}_{3} ; \delta$ (ppm). 


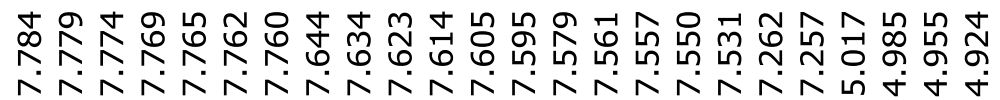

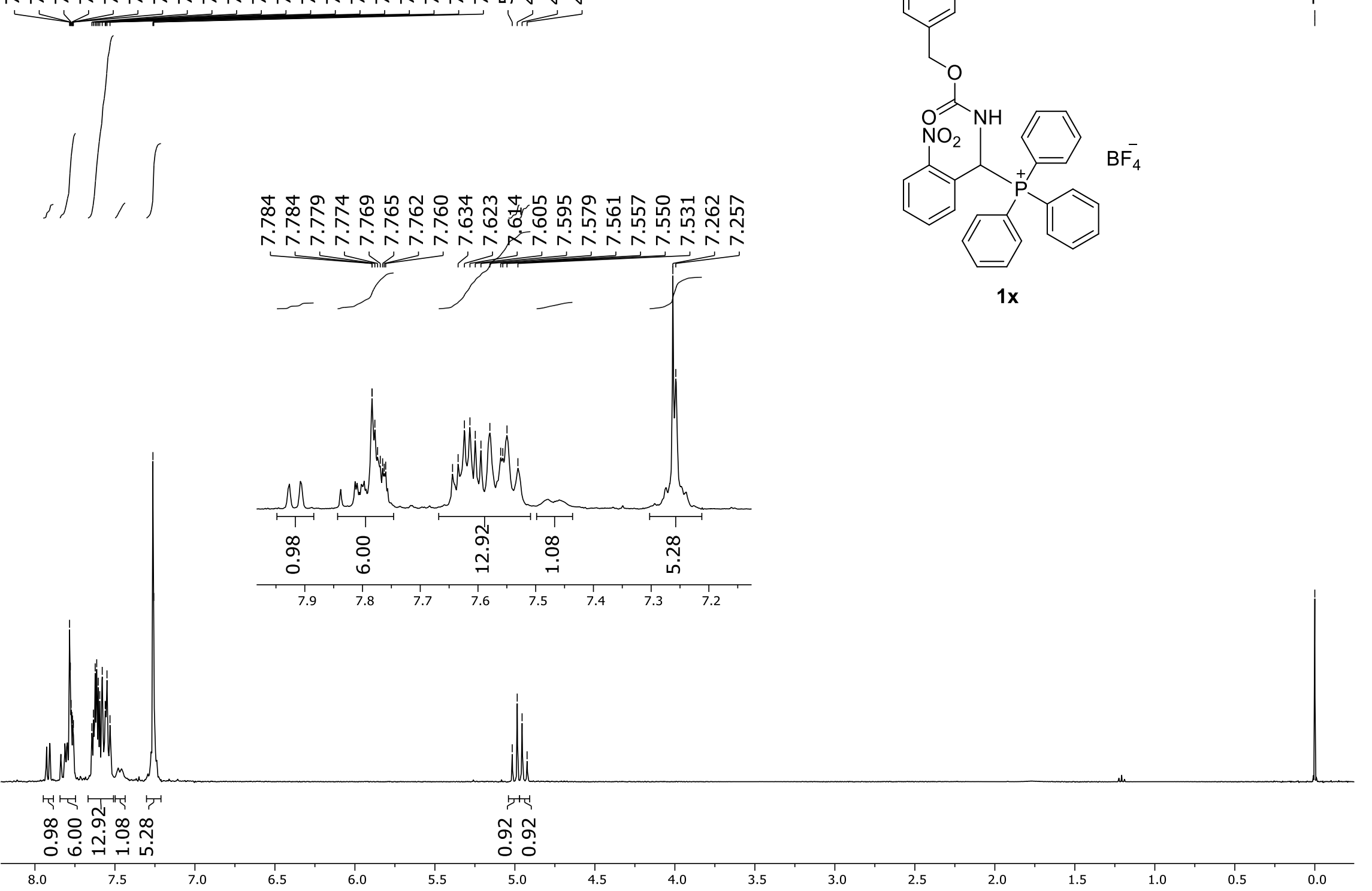

${ }^{1} \mathrm{H}$ NMR spectrum of 1-(N-benzyloxycarbonylamino)-1-(2-nitrophenyl)methyltriphenylphosphonium tetrafluoroborate (1x); $400 \mathrm{MHz} / \mathrm{CDCl} / 3 / \mathrm{TMS} ; \delta$ (ppm). 

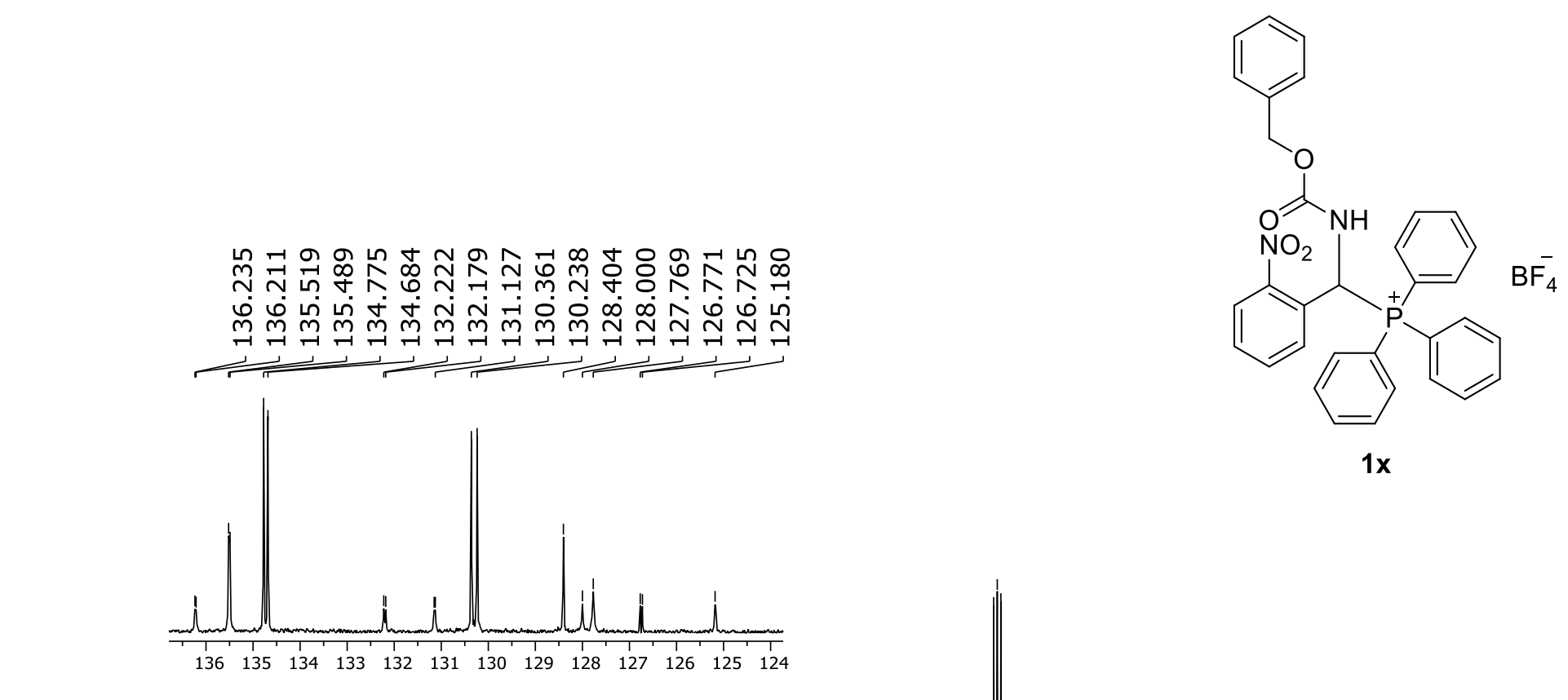

$1 \mathrm{x}$

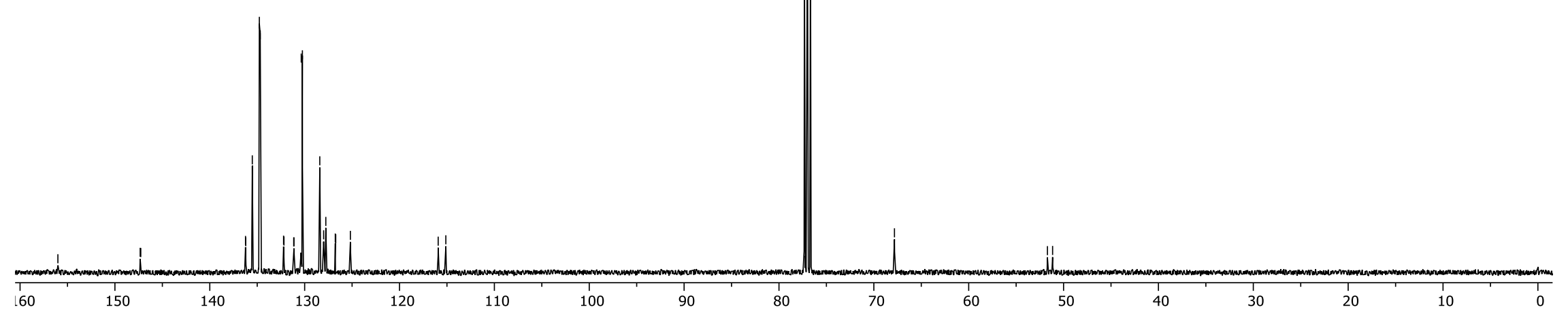

${ }^{13} \mathrm{C}\left\{{ }^{1} \mathrm{H}\right\}$ NMR spectrum of 1-(N-benzyloxycarbonylamino)-1-(2-nitrophenyl)methyltriphenylphosphonium tetrafluoroborate (1x); $100 \mathrm{MHz} / \mathrm{CDCl} / \mathrm{TMS} ; \delta$ (ppm). 

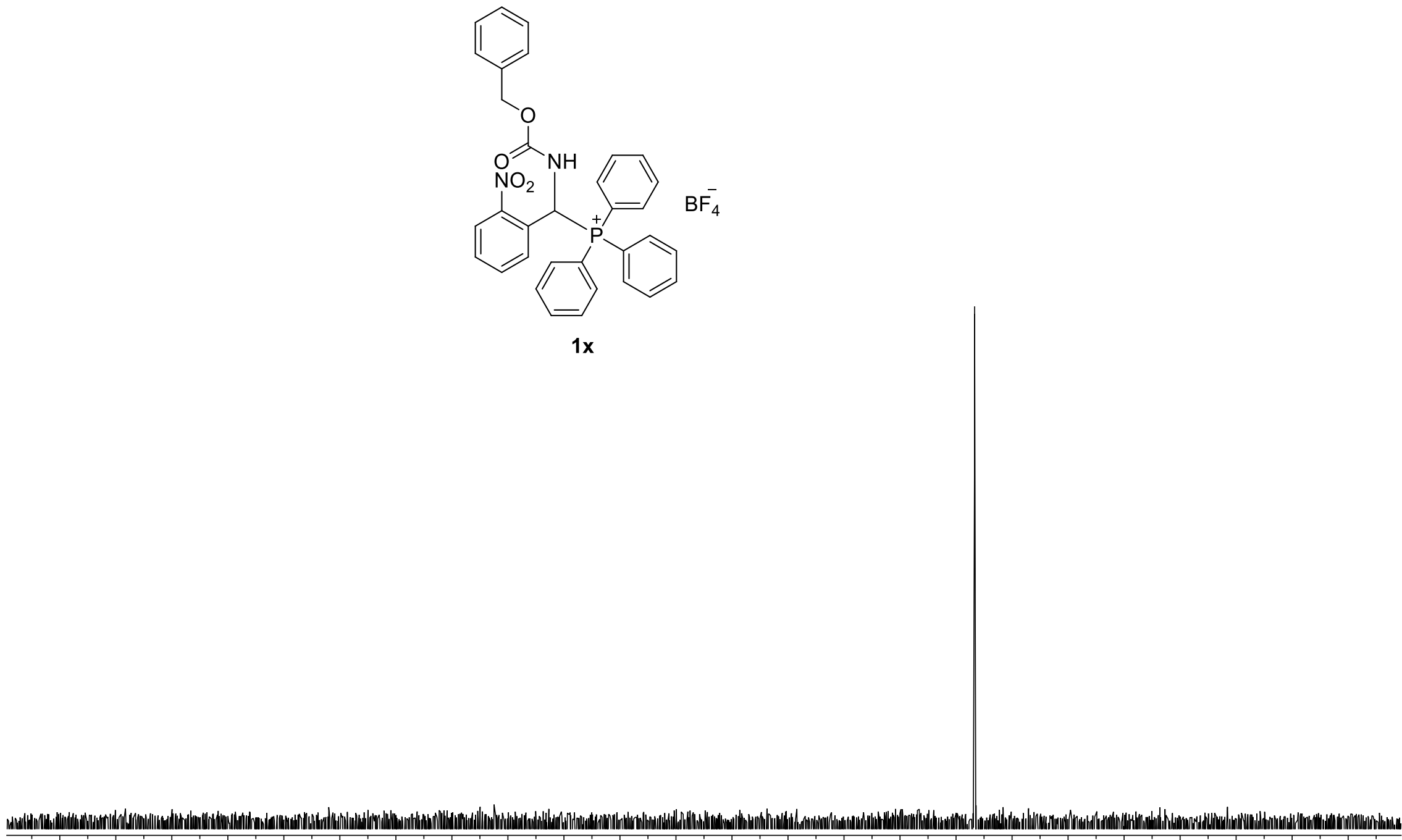

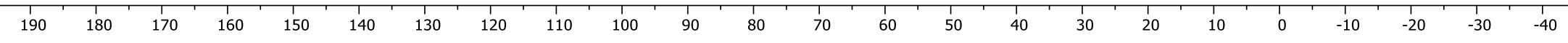

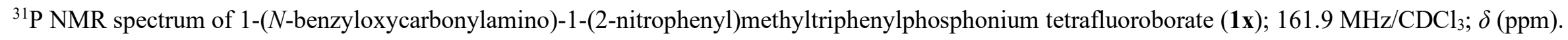


苦

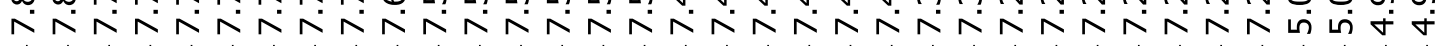
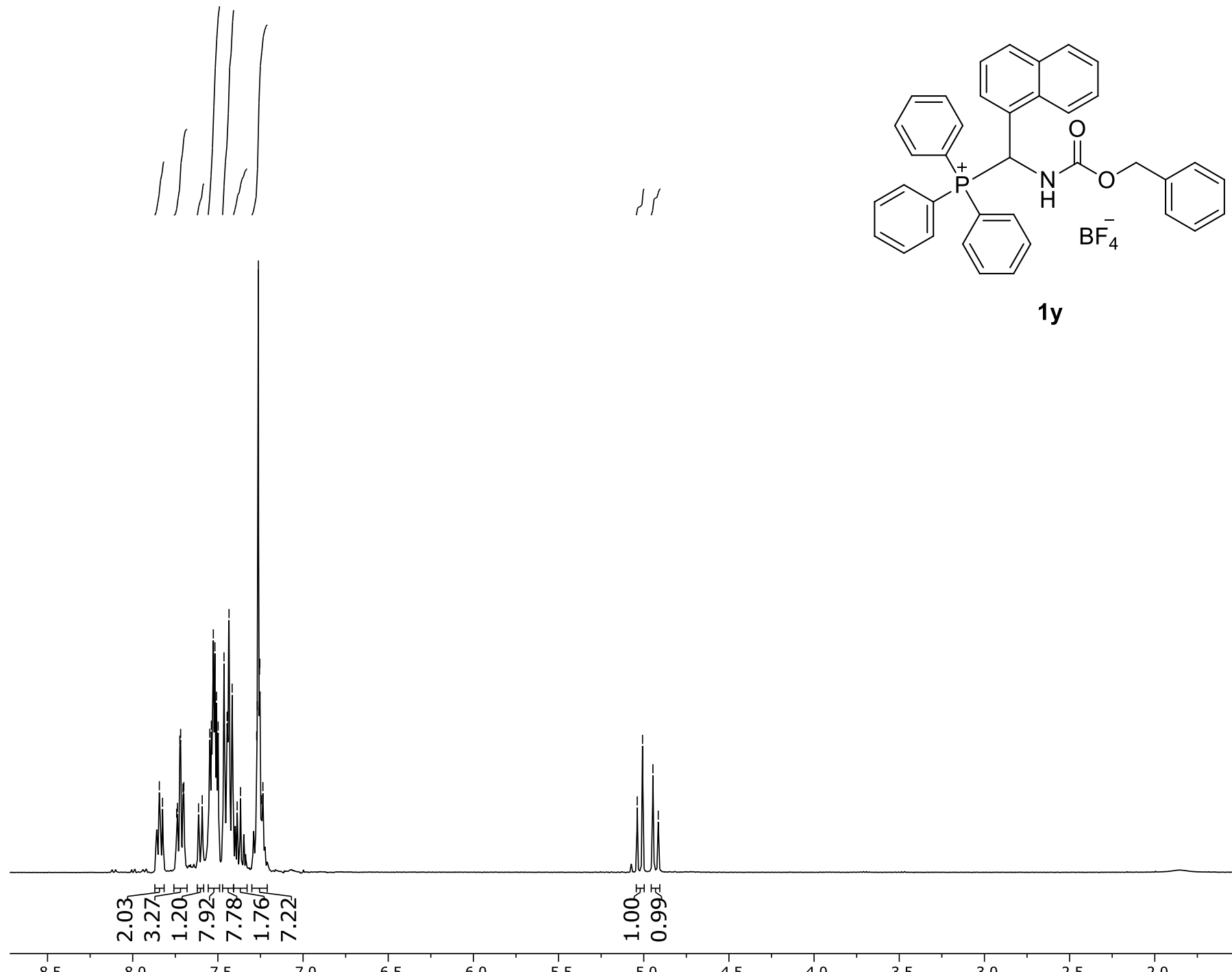

1y

${ }^{1} \mathrm{H}$ NMR spectrum of 1-(N-benzyloxycarbonylamino)-1-(1-naphthyl)methyltriphenylphosphonium tetrafluoroborate $(\mathbf{1 y}) ; 400 \mathrm{MHz} / \mathrm{CDCl} 3 / \mathrm{TMS} ; \delta$ (ppm). 


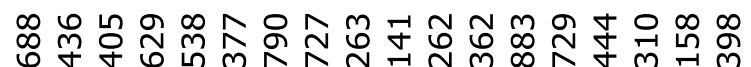

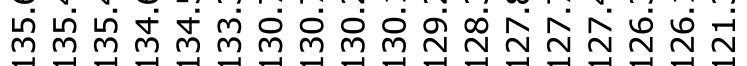
न नै जन
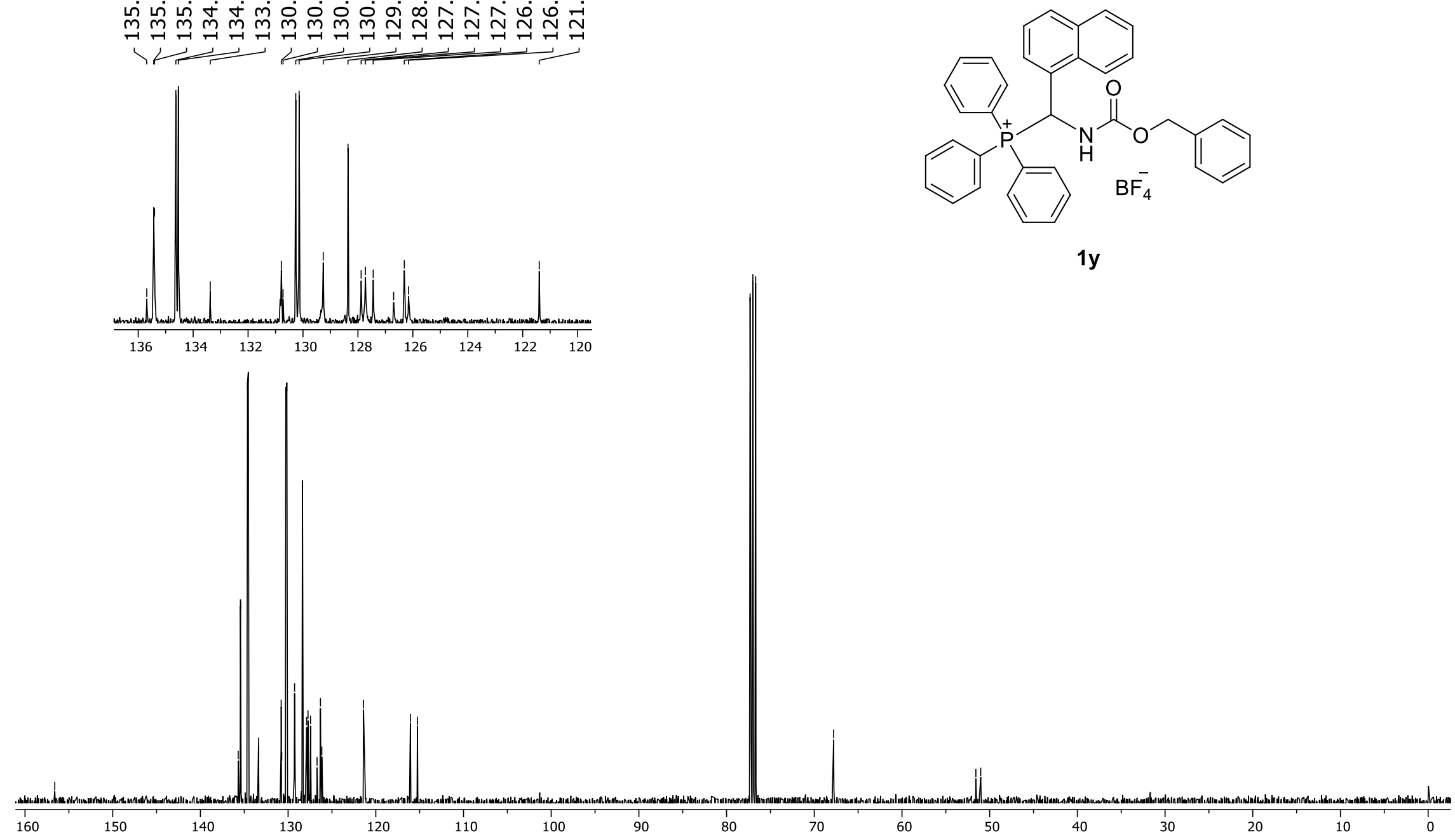

${ }^{13} \mathrm{C}\left\{{ }^{1} \mathrm{H}\right\}$ NMR spectrum of 1-(N-benzyloxycarbonylamino)-1-(1-naphthyl)methyltriphenylphosphonium tetrafluoroborate (1y); $100 \mathrm{MHz} / \mathrm{CDCl} / 3 / \mathrm{TMS} ; \delta$ (ppm). 


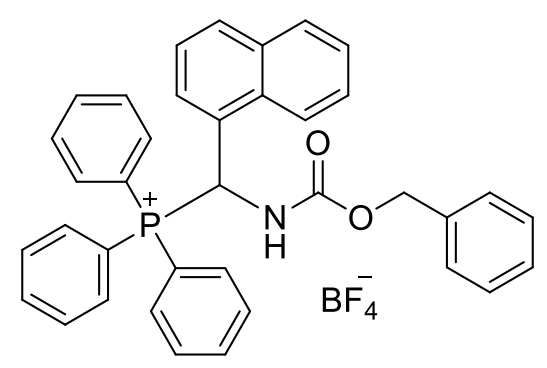

$1 y$
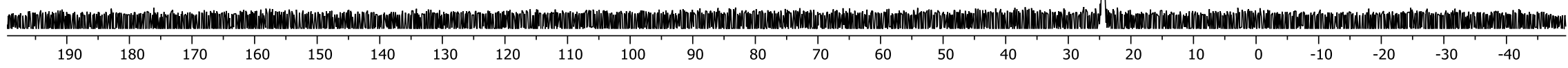

${ }^{31} \mathrm{P}$ NMR spectrum of 1-(N-benzyloxycarbonylamino)-1-(1-naphthyl)methyltriphenylphosphonium tetrafluoroborate (1y); $161.9 \mathrm{MHz} / \mathrm{CDCl}{ }_{3} ; \delta$ (ppm). 

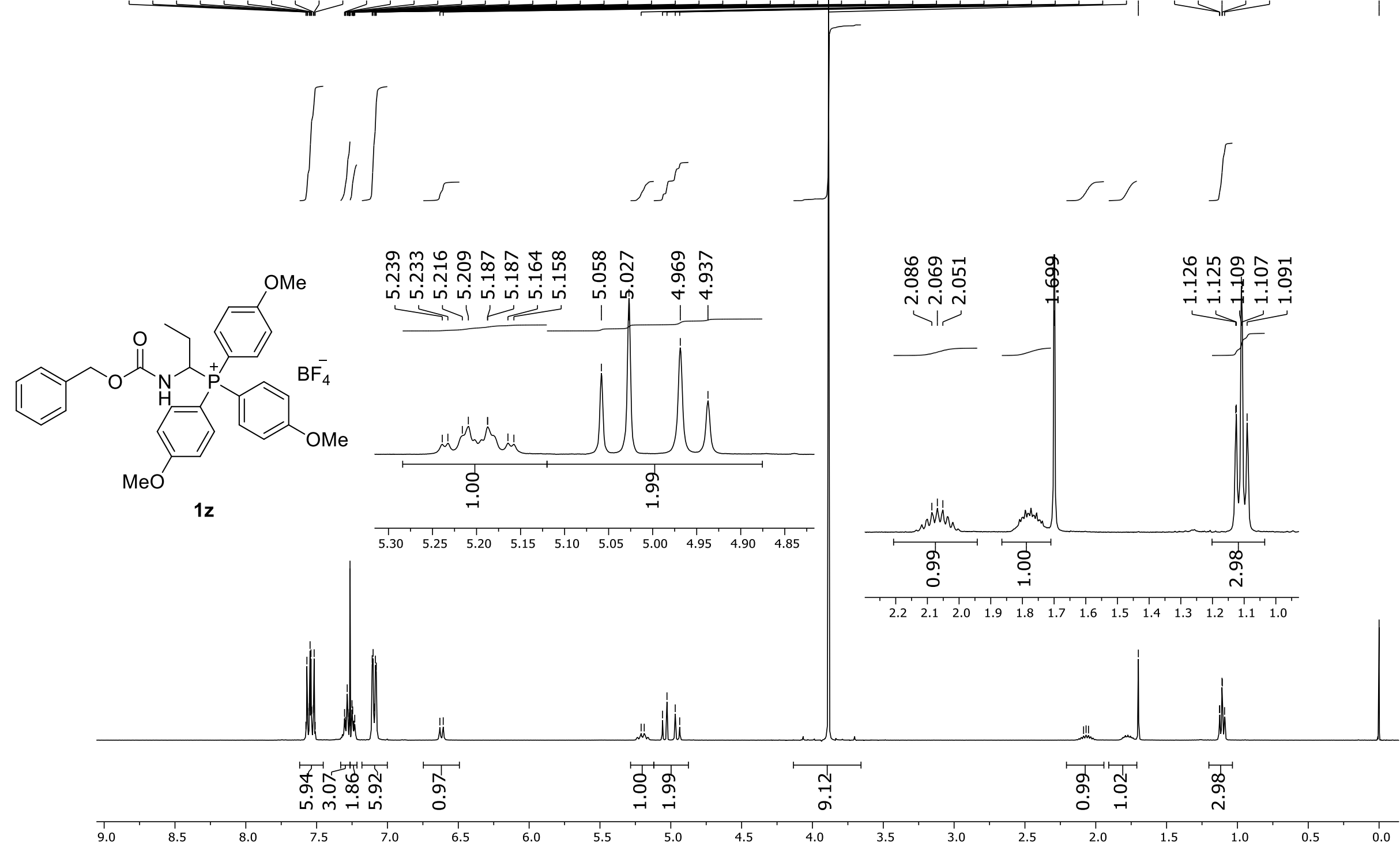

${ }^{1} \mathrm{H}$ NMR spectrum of 1-( $N$-benzyloxycarbonylamino)propyltris(4-methoxyphenyl)phosphonium tetrafluoroborate (1z); $400 \mathrm{MHz} / \mathrm{CDCl} 3 / \mathrm{TMS} ; \delta$ (ppm). 


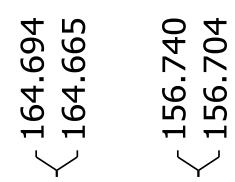

mo.

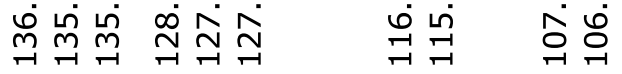

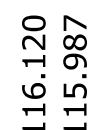

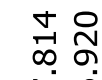
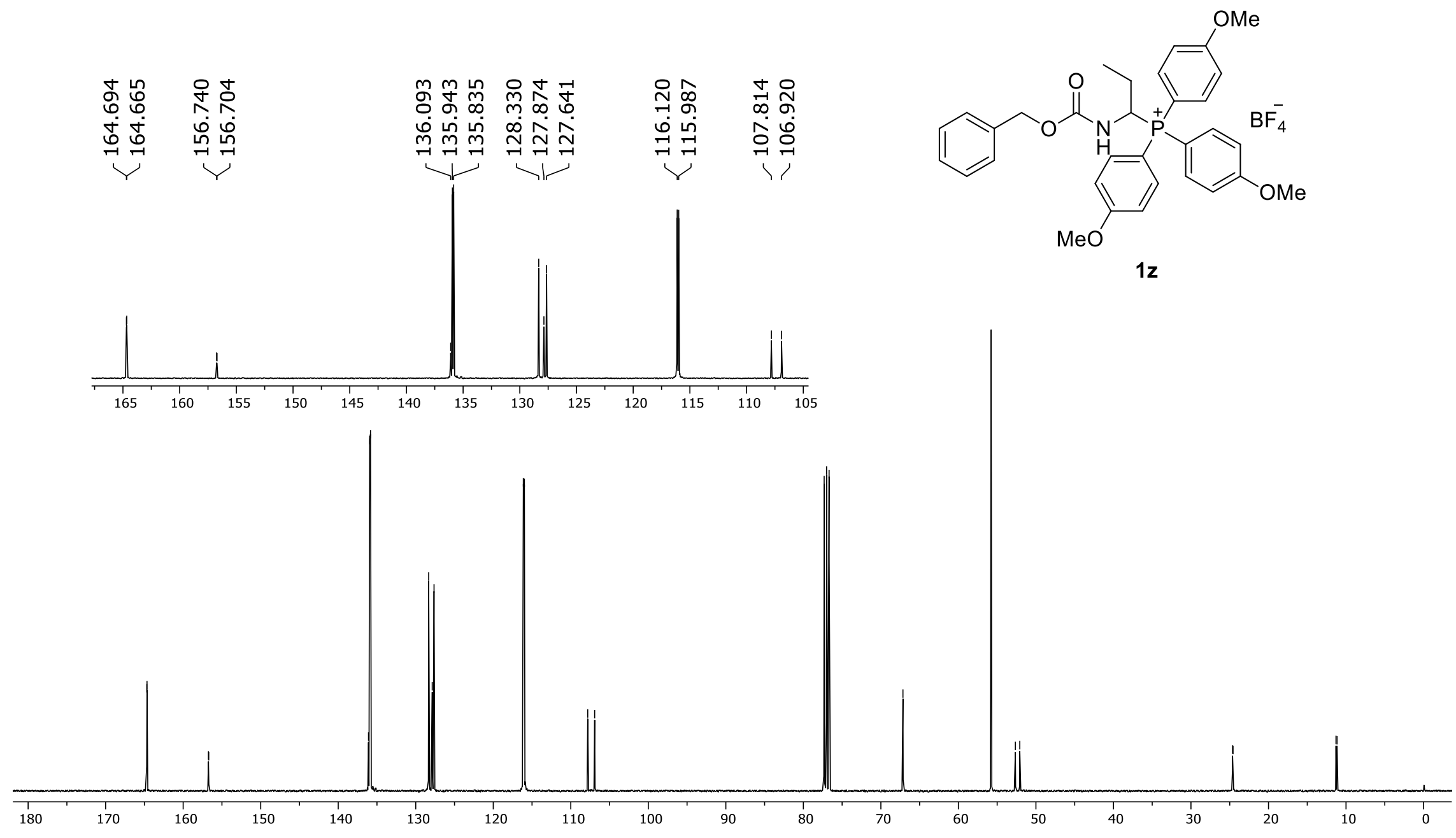

${ }^{13} \mathrm{C}\left\{{ }^{1} \mathrm{H}\right\}$ NMR spectrum of 1-(N-benzyloxycarbonylamino)propyltris(4-methoxyphenyl)phosphonium tetrafluoroborate $(\mathbf{1 z}) ; 100 \mathrm{MHz} / \mathrm{CDCl} / 3 / \mathrm{TMS} ; \delta$ (ppm). 


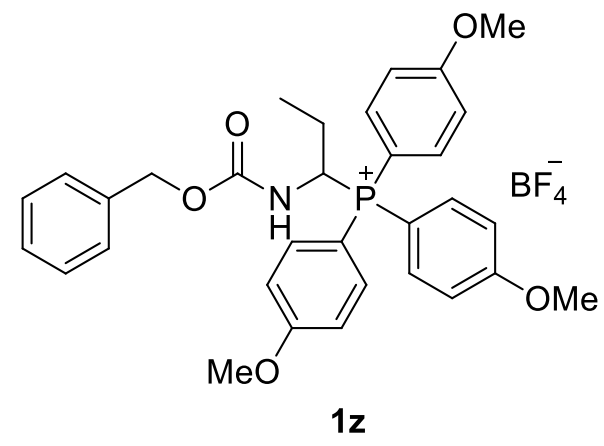

190 180 


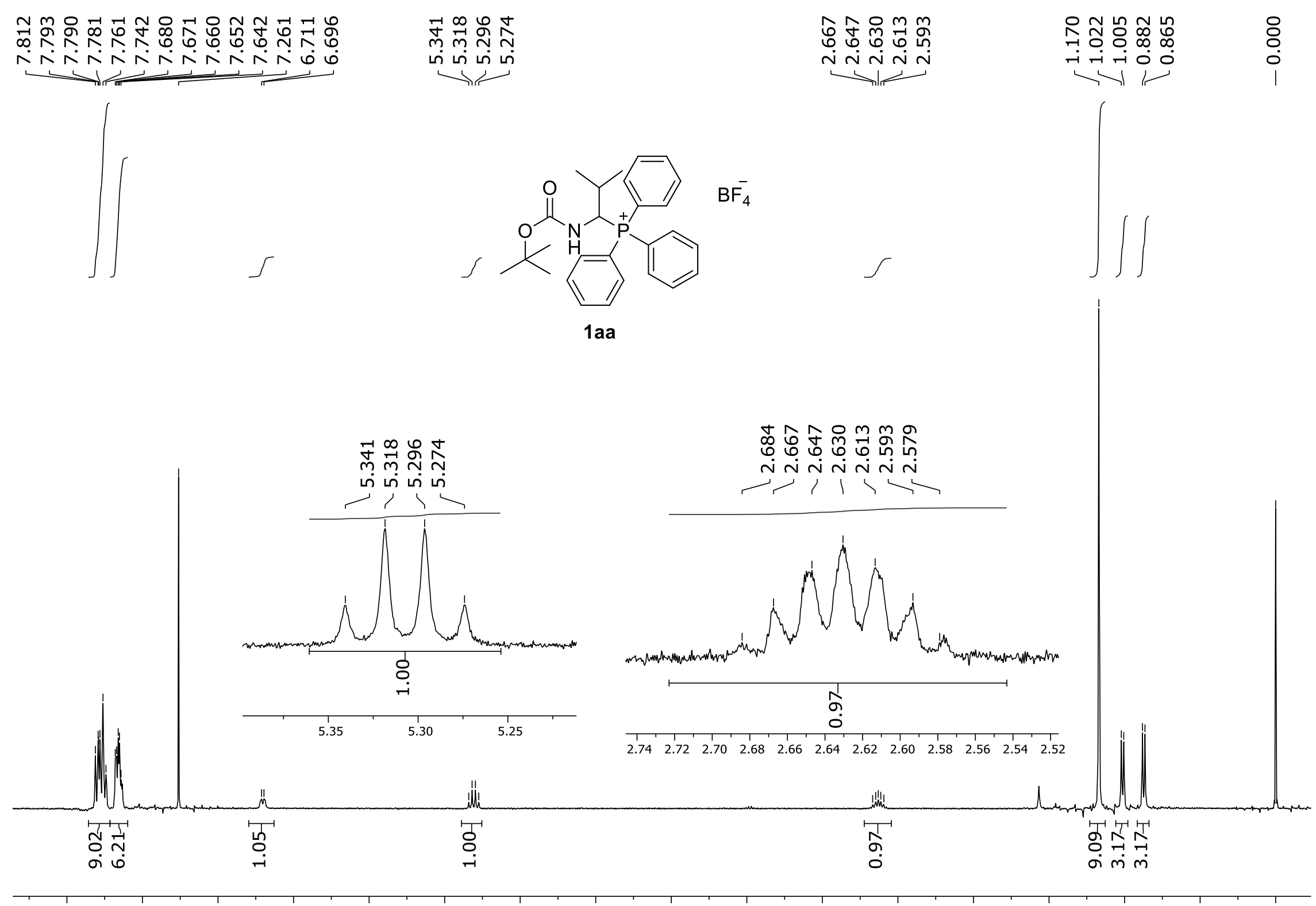

${ }^{1} \mathrm{H}$ NMR spectrum of 1-( $N$-tert-butoxycarbonylamino)-2-methylpropyltriphenylphosphonium tetrafluoroborate (1aa); $400 \mathrm{MHz} / \mathrm{CDCl} 3 / \mathrm{TMS} ; \delta$ (ppm). 

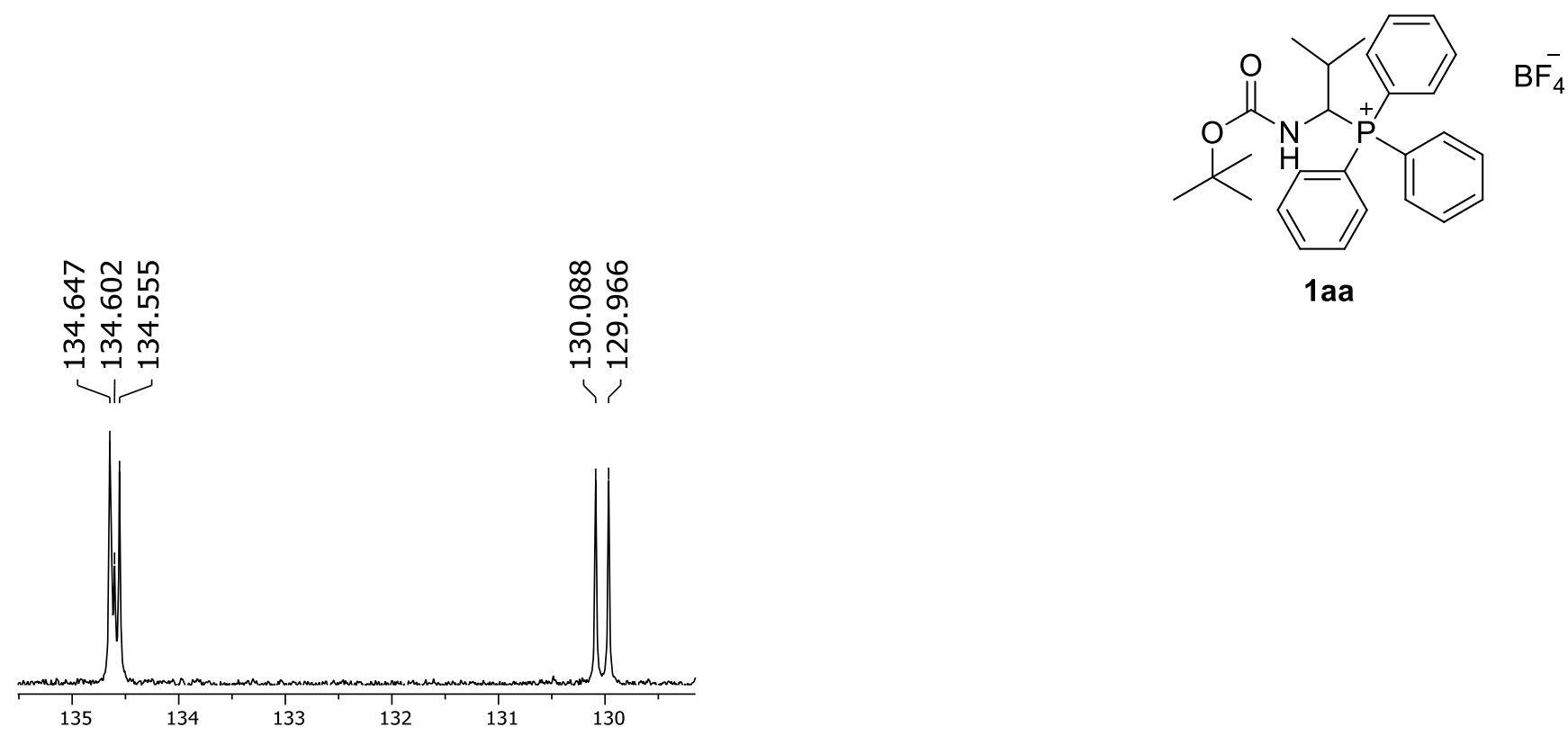

1 aa

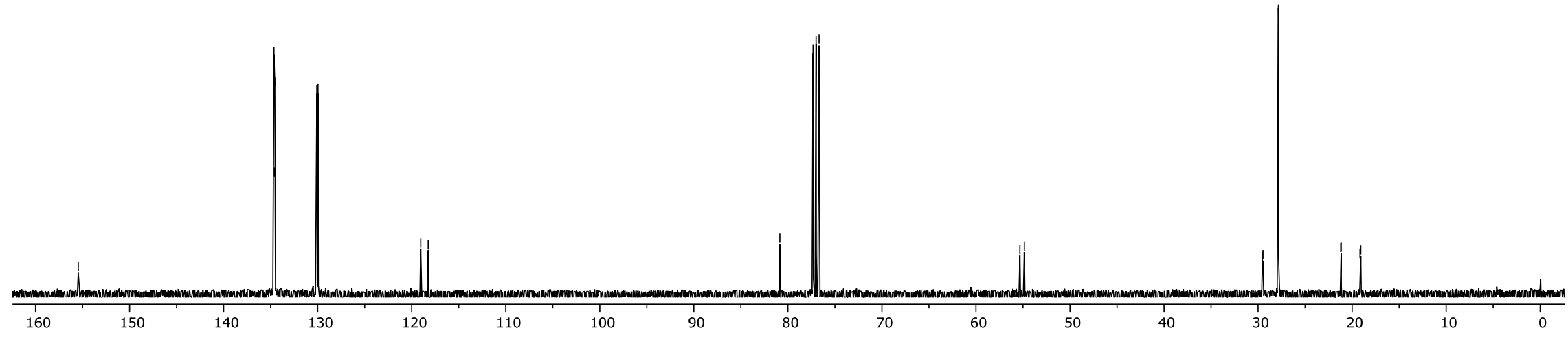

${ }^{13} \mathrm{C}\left\{{ }^{1} \mathrm{H}\right\}$ NMR spectrum of 1-(N-tert-butoxycarbonylamino)-2-methylpropyltriphenylphosphonium tetrafluoroborate (1aa); $100 \mathrm{MHz} / \mathrm{CDCl} / \mathrm{TMS} ; \delta$ (ppm). 


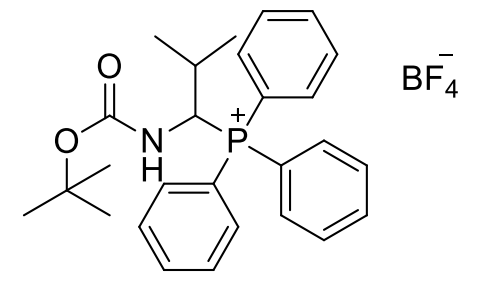

1 aa

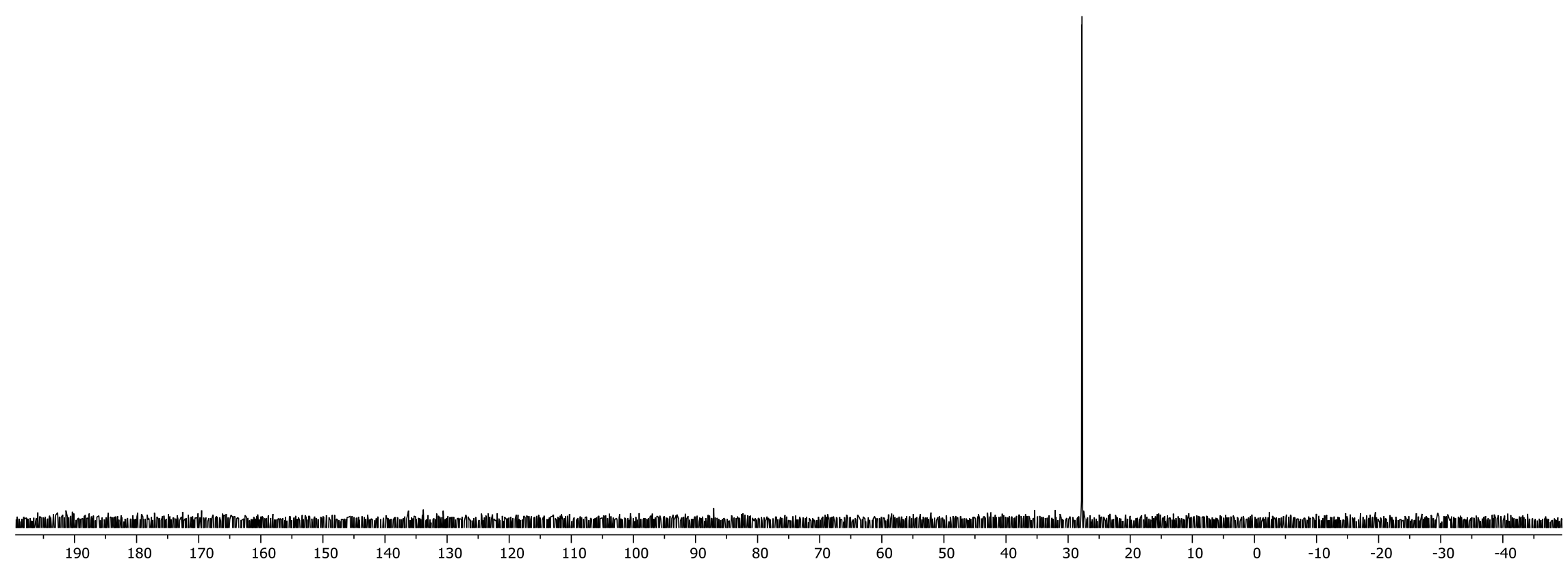

${ }^{31} \mathrm{P}$ NMR spectrum of 1-( $N$-tert-butoxycarbonylamino)-2-methylpropyltriphenylphosphonium tetrafluoroborate $(\mathbf{1 a a}) ; 161.9 \mathrm{MHz} / \mathrm{CDCl}_{3} ; \delta(\mathrm{ppm})$. 


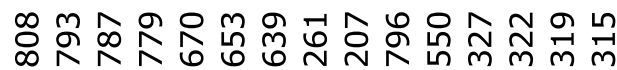

ヘヘヘヘヘヘヘヘ
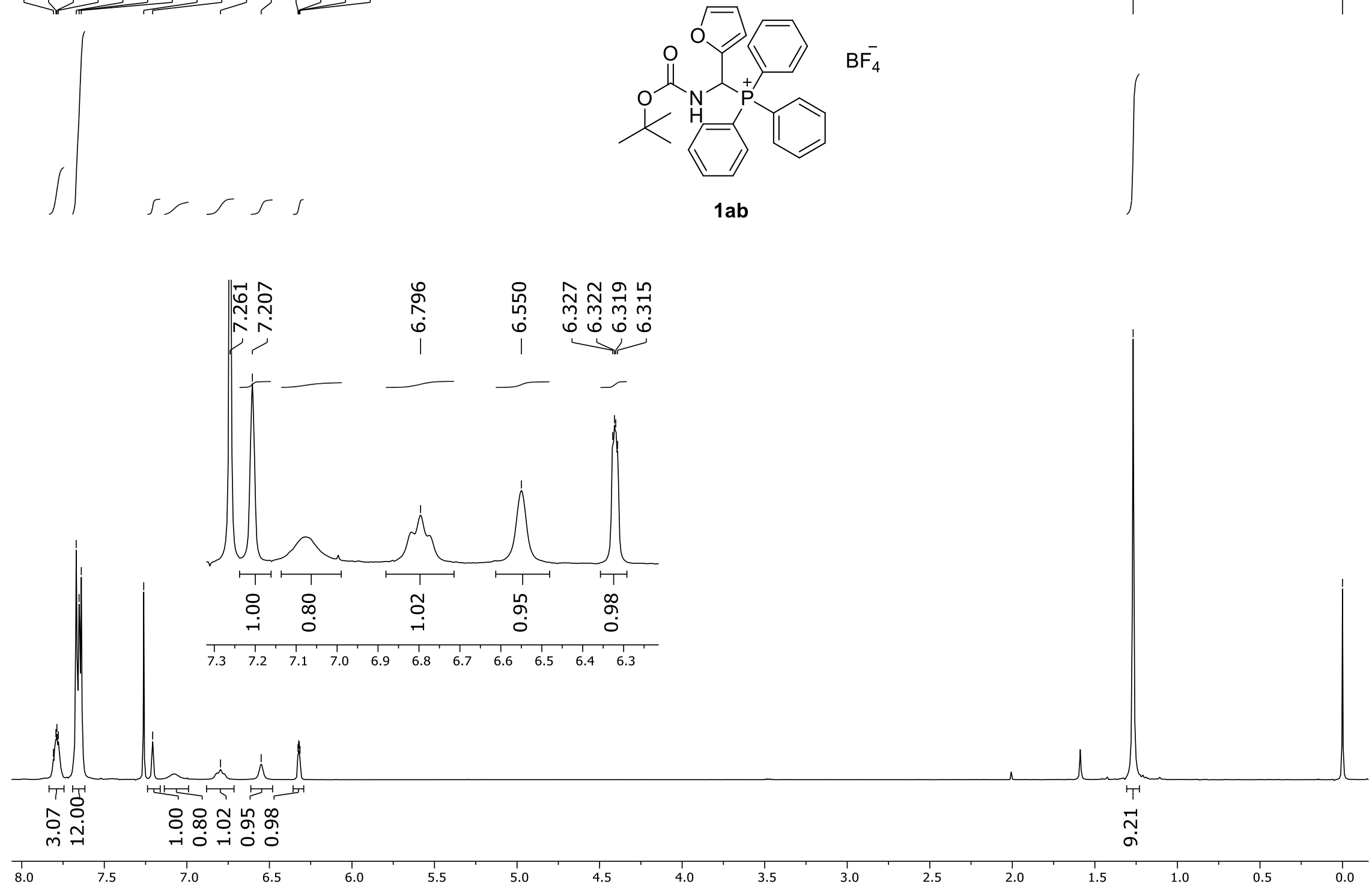

${ }^{1} \mathrm{H}$ NMR spectrum of 1-( $N$-tert-butoxycarbonylamino)-1-(2-furyl)methyltriphenylphosphonium tetrafluoroborate (1ab); $400 \mathrm{MHz} / \mathrm{CDCl} / 3 \mathrm{TMS} ; \delta$ (ppm). 

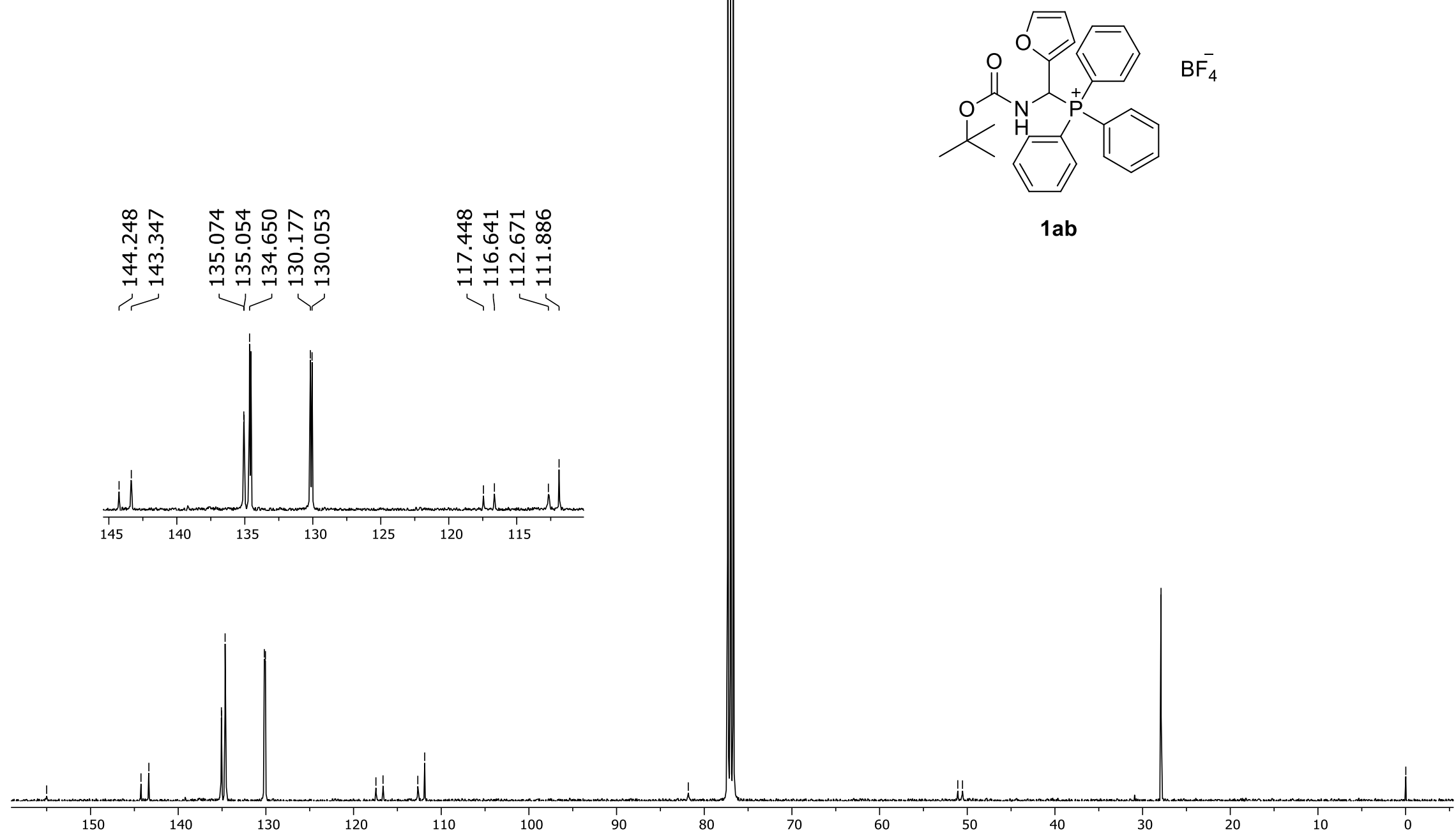

${ }^{13} \mathrm{C}\left\{{ }^{1} \mathrm{H}\right\}$ NMR spectrum of 1-( $N$-tert-butoxycarbonylamino)-1-(2-furyl)methyltriphenylphosphonium tetrafluoroborate (1ab); $100 \mathrm{MHz} / \mathrm{CDCl} / 3 / \mathrm{TMS} ; \delta$ (ppm). 


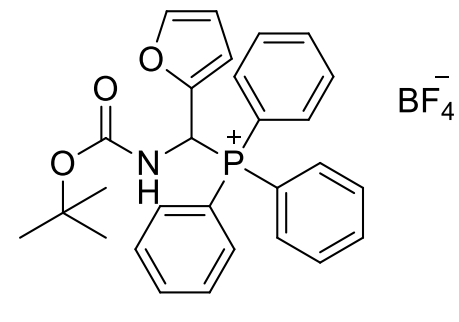

$1 a b$

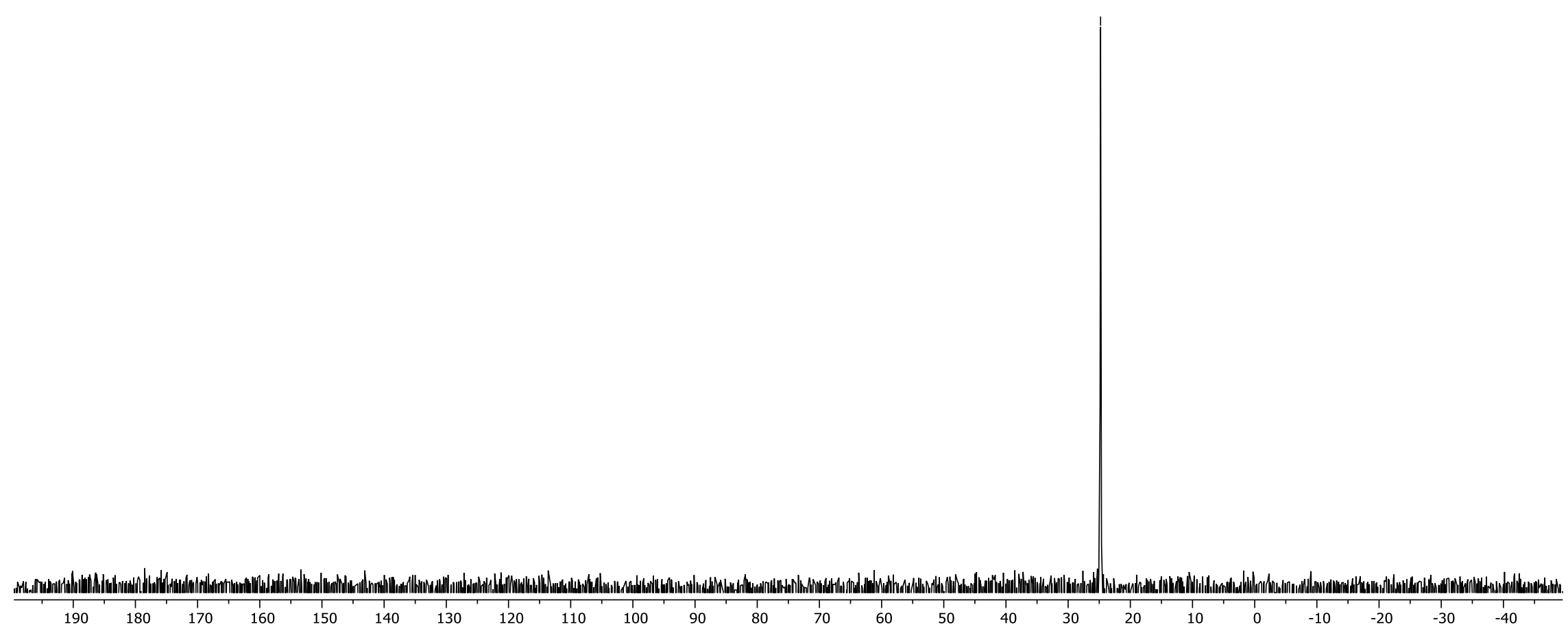

${ }^{31} \mathrm{P}$ NMR spectrum of 1-( $N$-tert-butoxycarbonylamino)-1-(2-furyl)methyltriphenylphosphonium tetrafluoroborate $(\mathbf{1 a b}) ; 161.9 \mathrm{MHz} / \mathrm{CDCl} 3 ; \delta(\mathrm{ppm})$ 


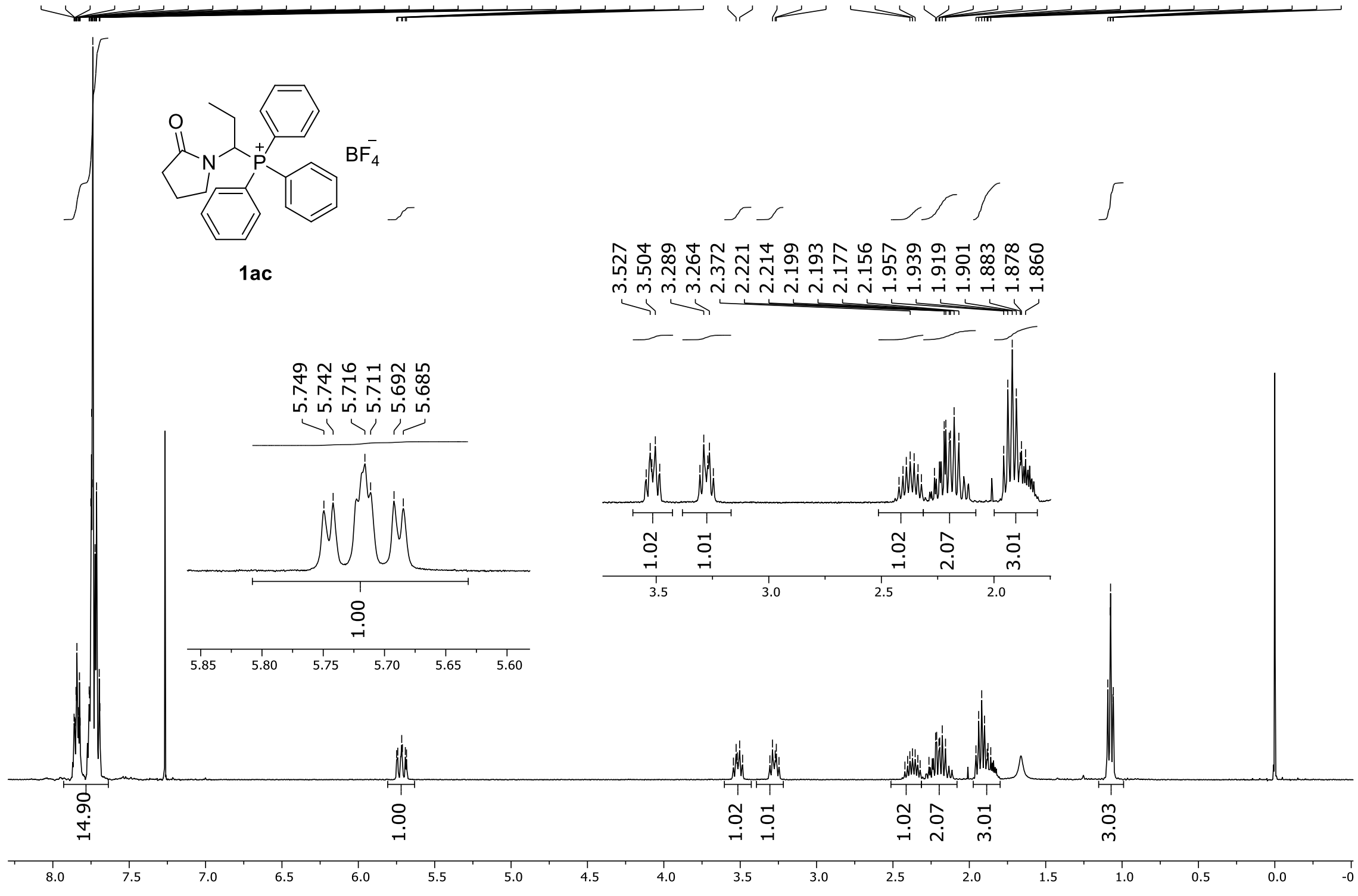

${ }^{1} \mathrm{H}$ NMR spectrum of 1-(2-oxopyrrolidin-1-yl)propyltriphenylphosphonium tetrafluoroborate (1ac); $400 \mathrm{MHz} / \mathrm{CDCl}_{3} / \mathrm{TMS} ; \delta$ (ppm). 


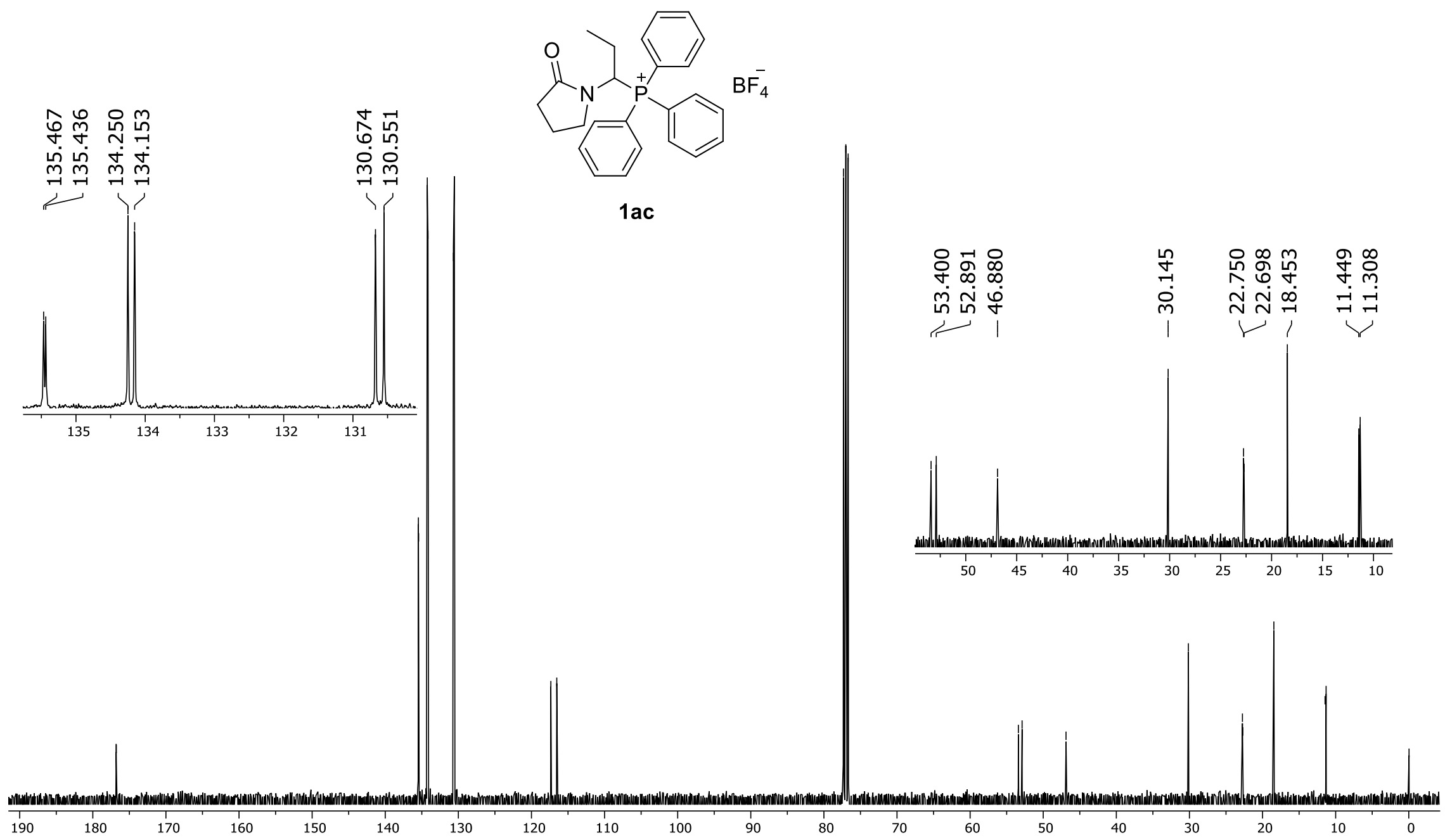

${ }^{13} \mathrm{C}\left\{{ }^{1} \mathrm{H}\right\}$ NMR spectrum of 1-(2-oxopyrrolidin-1-yl)propyltriphenylphosphonium tetrafluoroborate (1ac); $100 \mathrm{MHz} / \mathrm{CDCl}_{3} / \mathrm{TMS} ; \delta$ (ppm). 


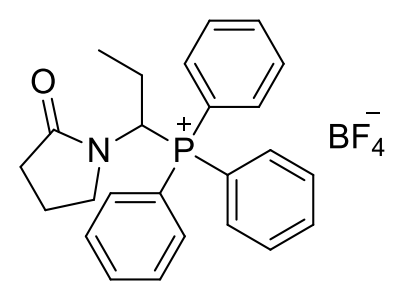

1 ac

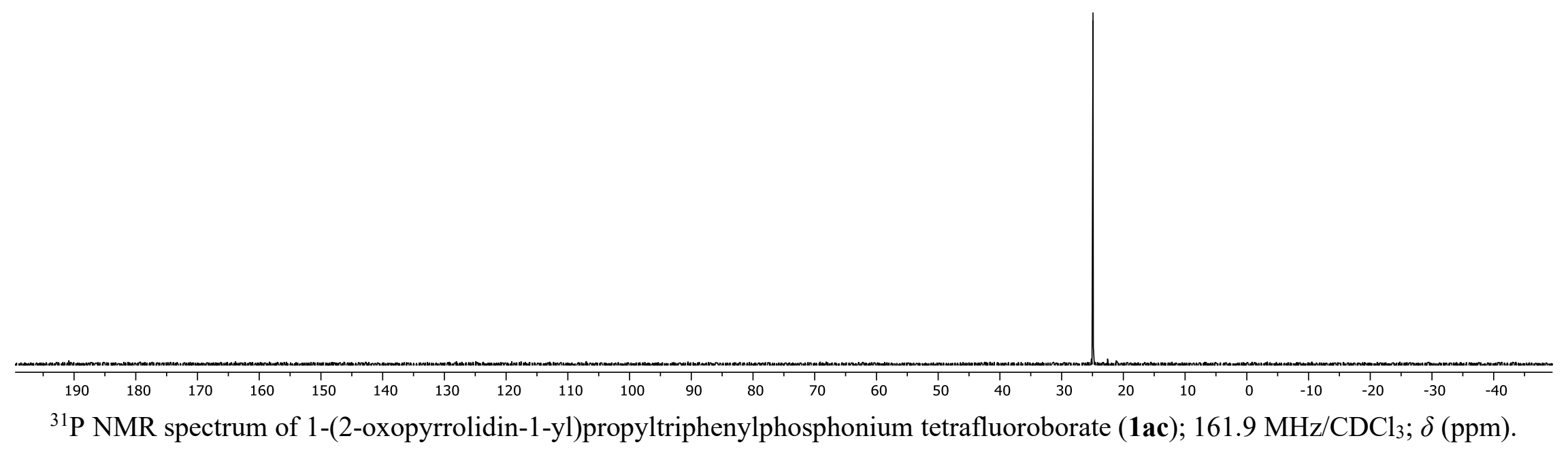




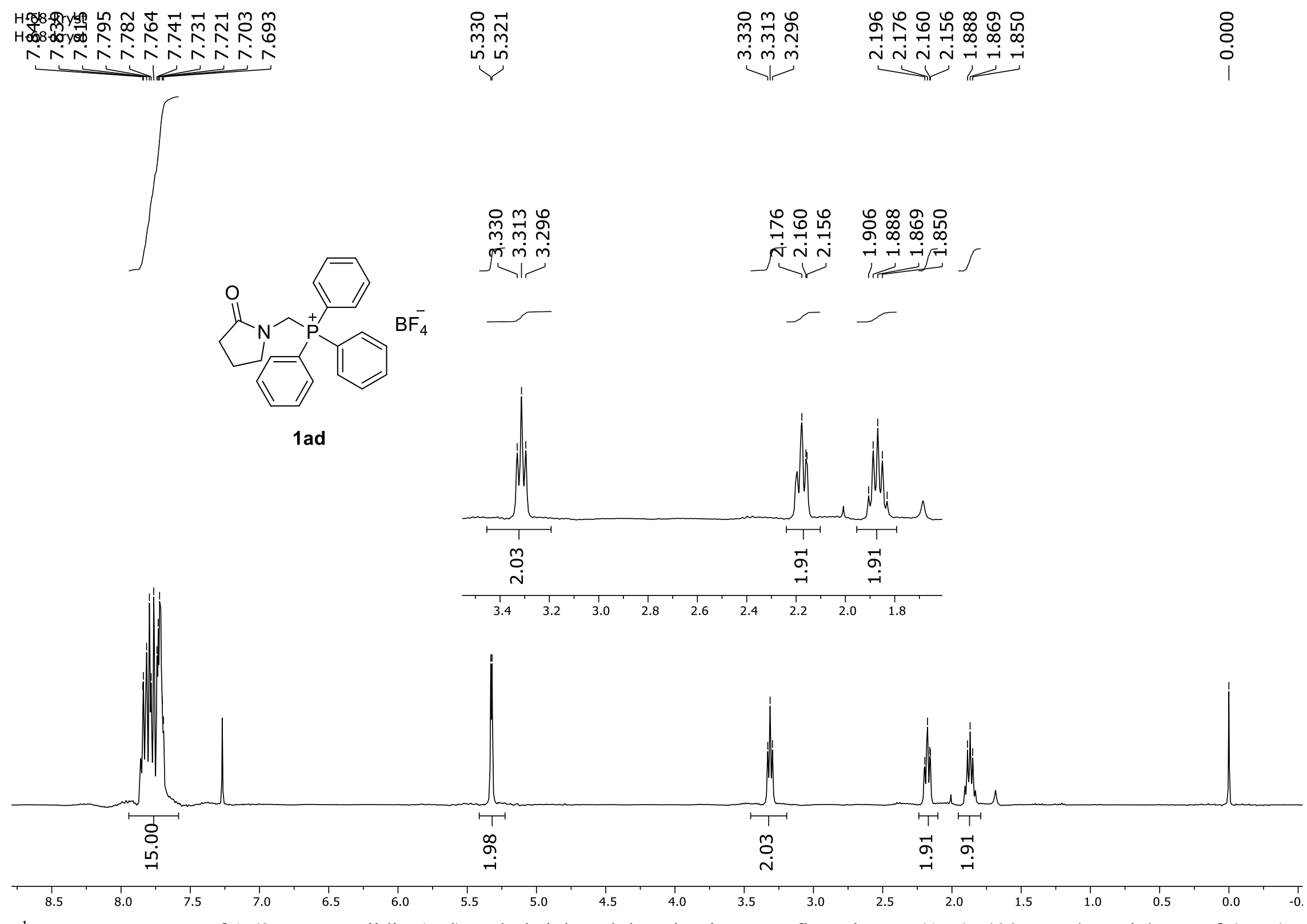

${ }^{1} \mathrm{H}$ NMR spectrum of 1-(2-oxopyrrolidin-1-yl)methyltriphenylphosphonium tetrafluoroborate (1ad); $400 \mathrm{MHz} / \mathrm{CDCl} / \mathrm{TMS} ; \delta$ (ppm). 


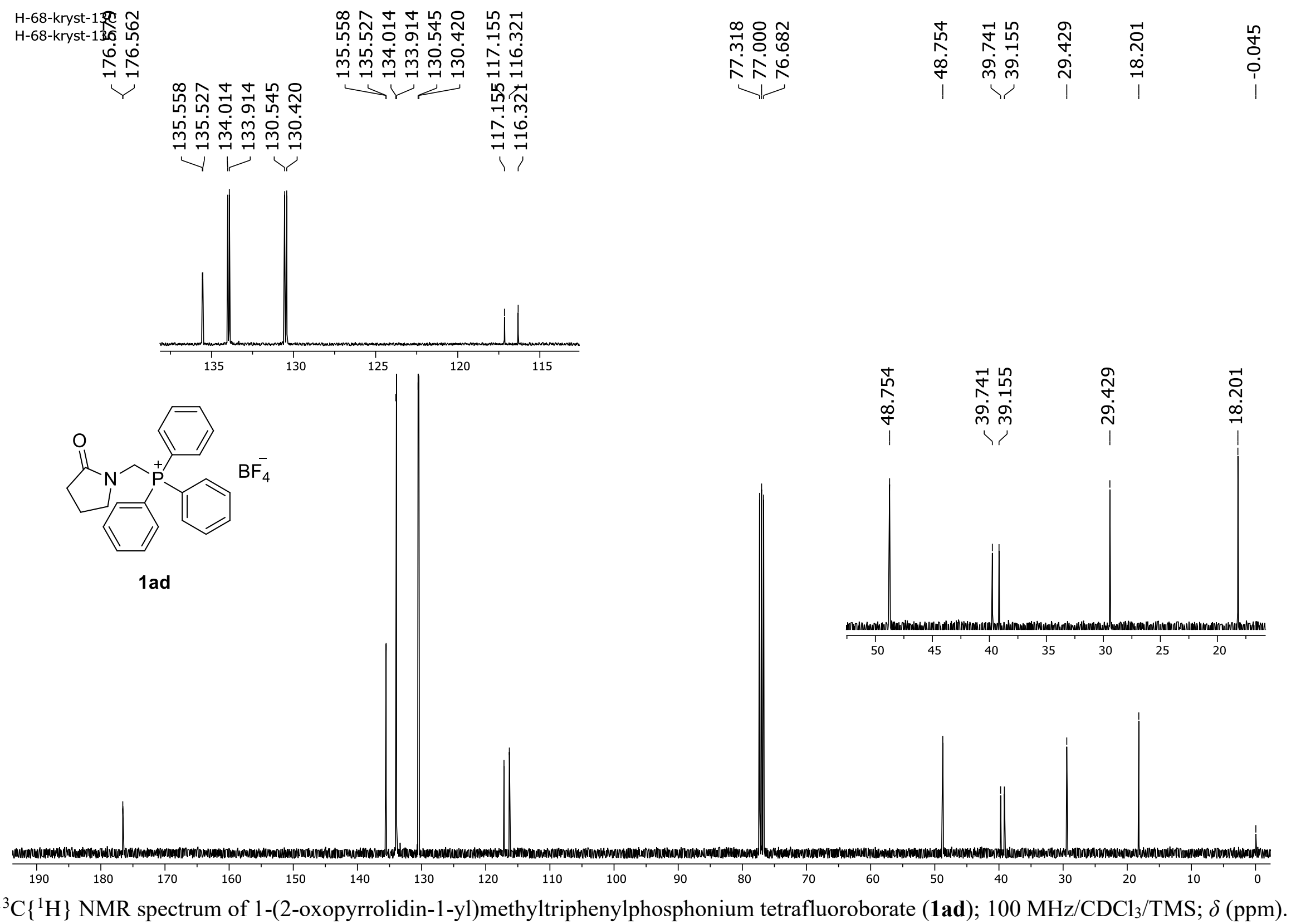




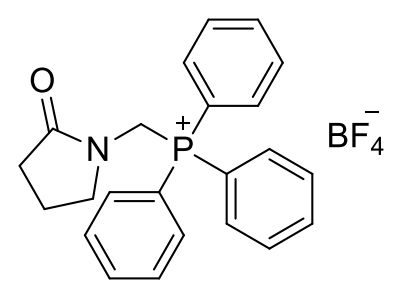

$1 \mathrm{ad}$

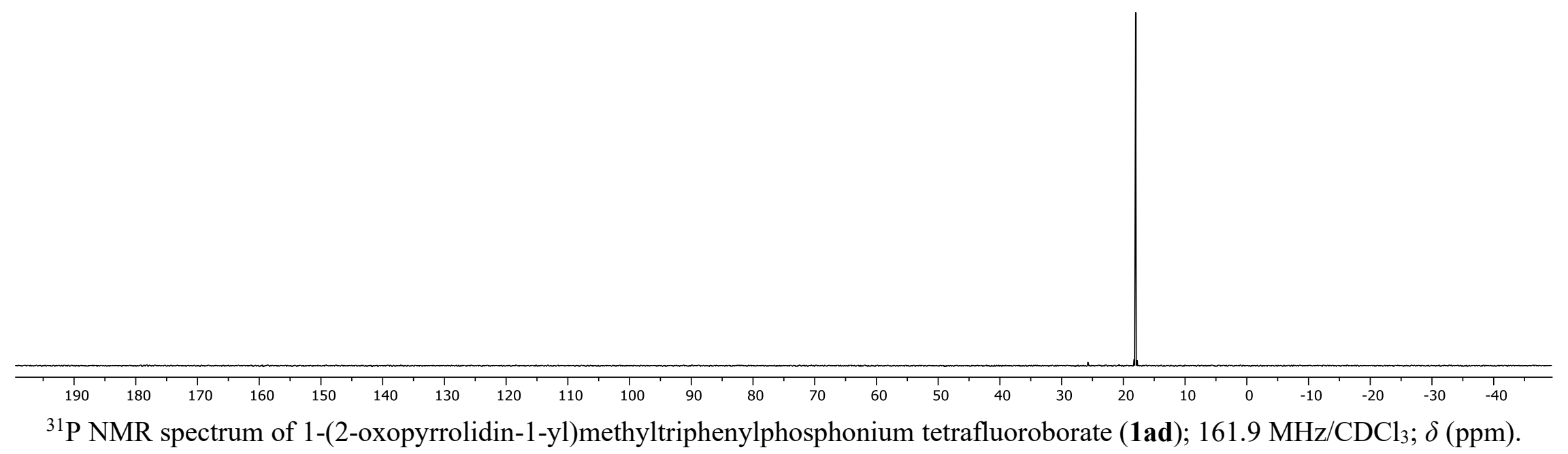




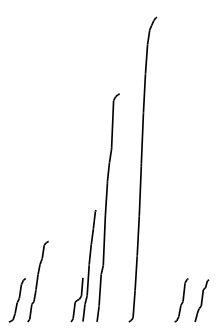

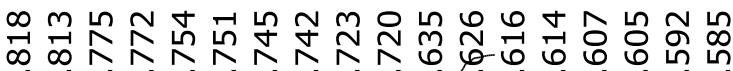

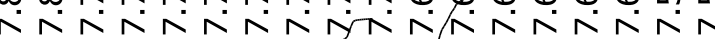
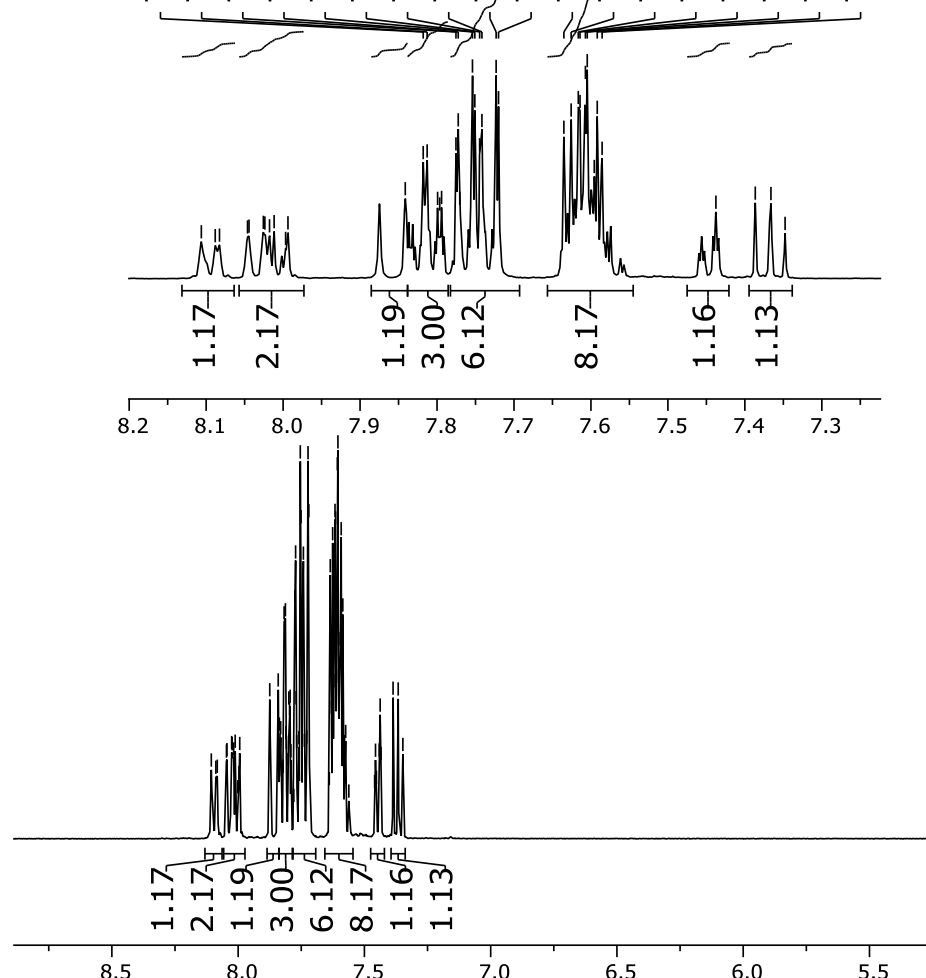

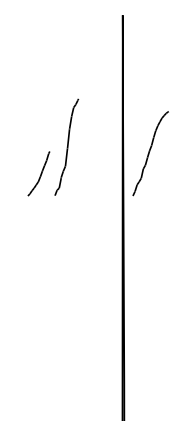

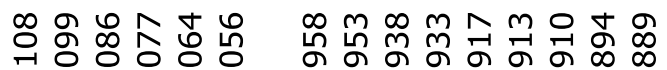

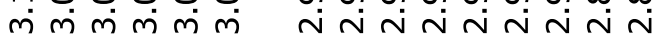
प1,

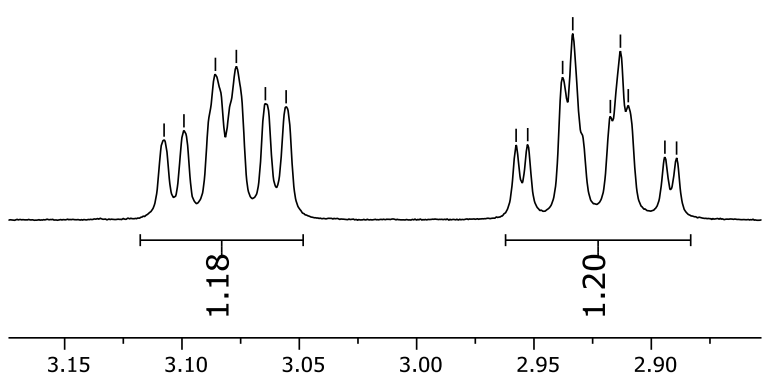

3.15
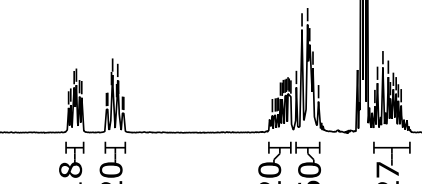

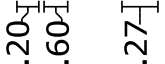

3.0

${ }^{1} \mathrm{H}$ NMR spectrum of 1-(2-oxopyrrolidin-1-yl)-1-(1-naphthyl)methyltriphenylphosphonium tetrafluoroborate (1ae); 400 MHz/CD 3 CN/TMS; $\delta$ (ppm). 


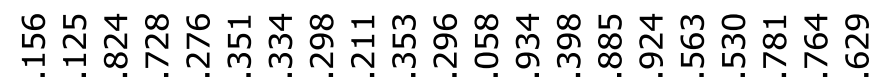

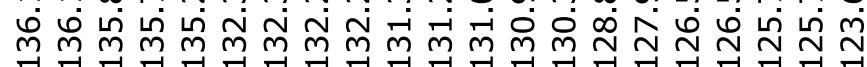

กิ๊ กิ
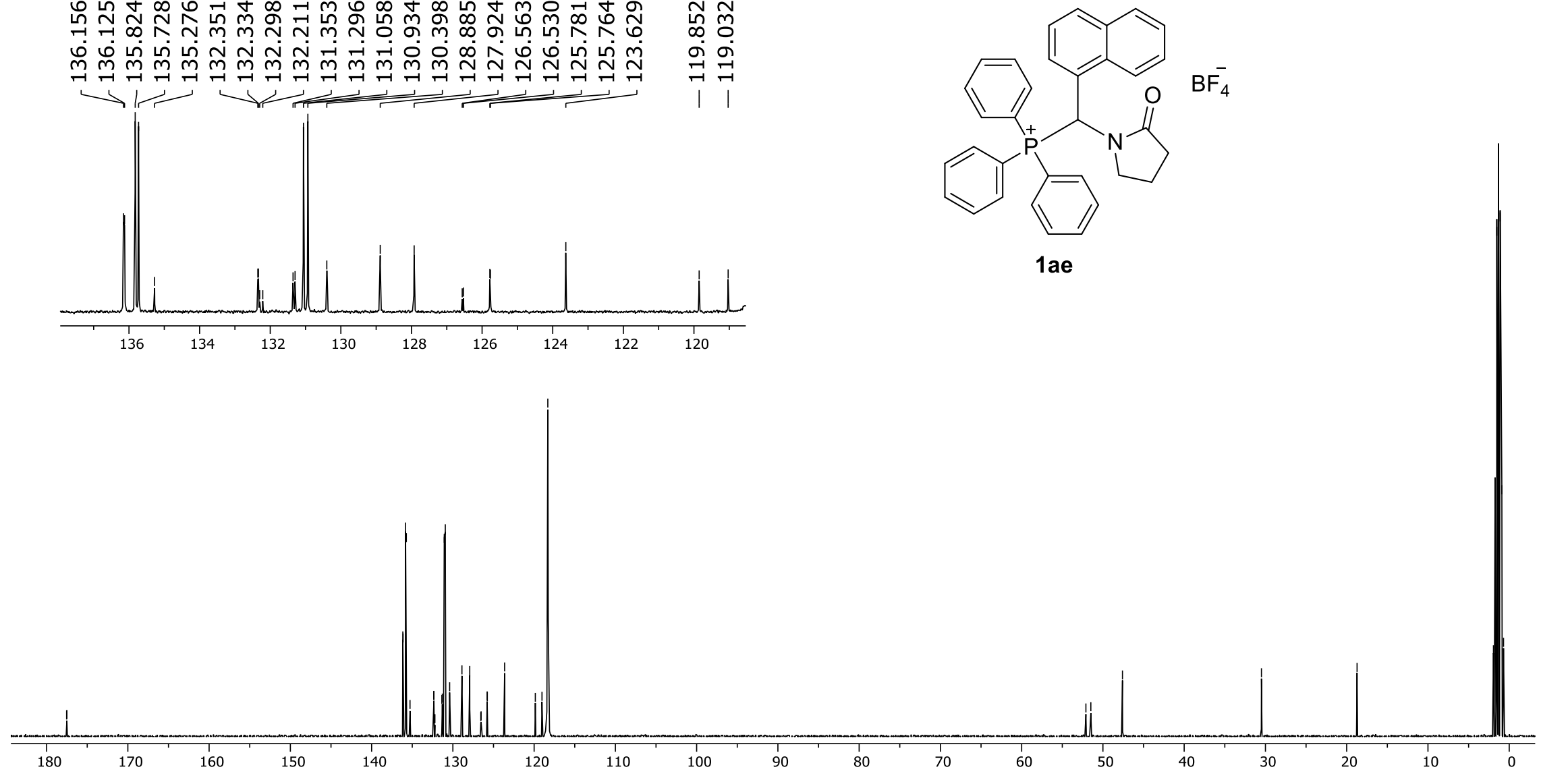

${ }^{13} \mathrm{C}\left\{{ }^{1} \mathrm{H}\right\}$ NMR spectrum of 1-(2-oxopyrrolidin-1-yl)-1-(1-naphthyl)methyltriphenylphosphonium tetrafluoroborate (1ae); $100 \mathrm{MHz} / \mathrm{CD}{ }_{3} \mathrm{CN} / \mathrm{TMS}$; $\delta(\mathrm{ppm})$. 


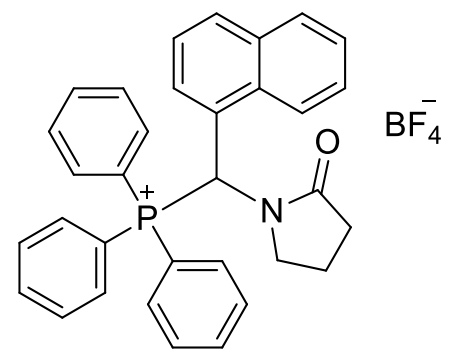

1 ae

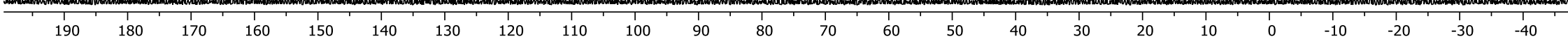

${ }^{31} \mathrm{P}$ NMR spectrum of 1-(2-oxopyrrolidin-1-yl)-1-(1-naphthyl)methyltriphenylphosphonium tetrafluoroborate (1ae); $161.9 \mathrm{MHz} / \mathrm{CD}{ }_{3} \mathrm{CN} ; \delta$ (ppm) 


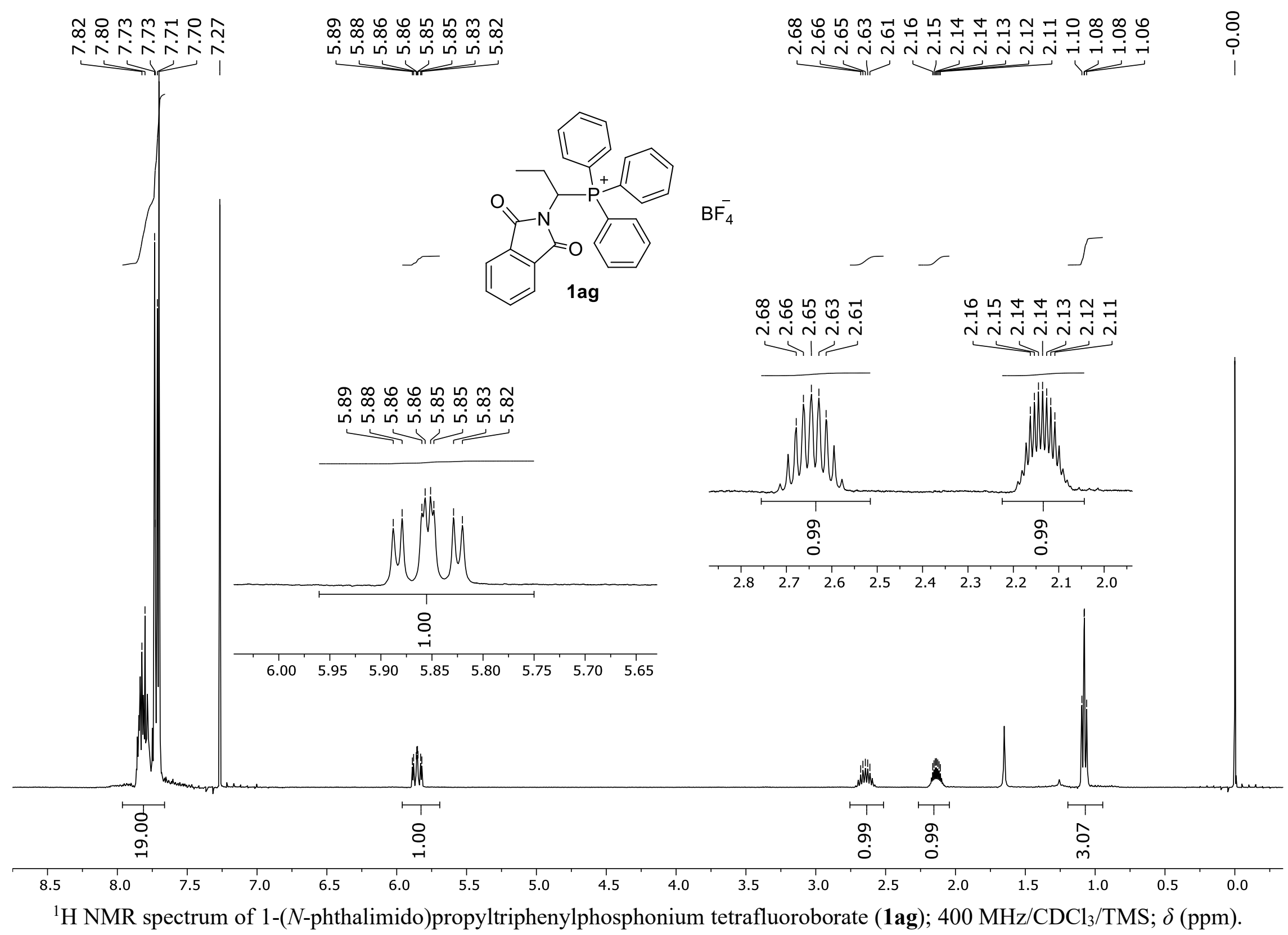




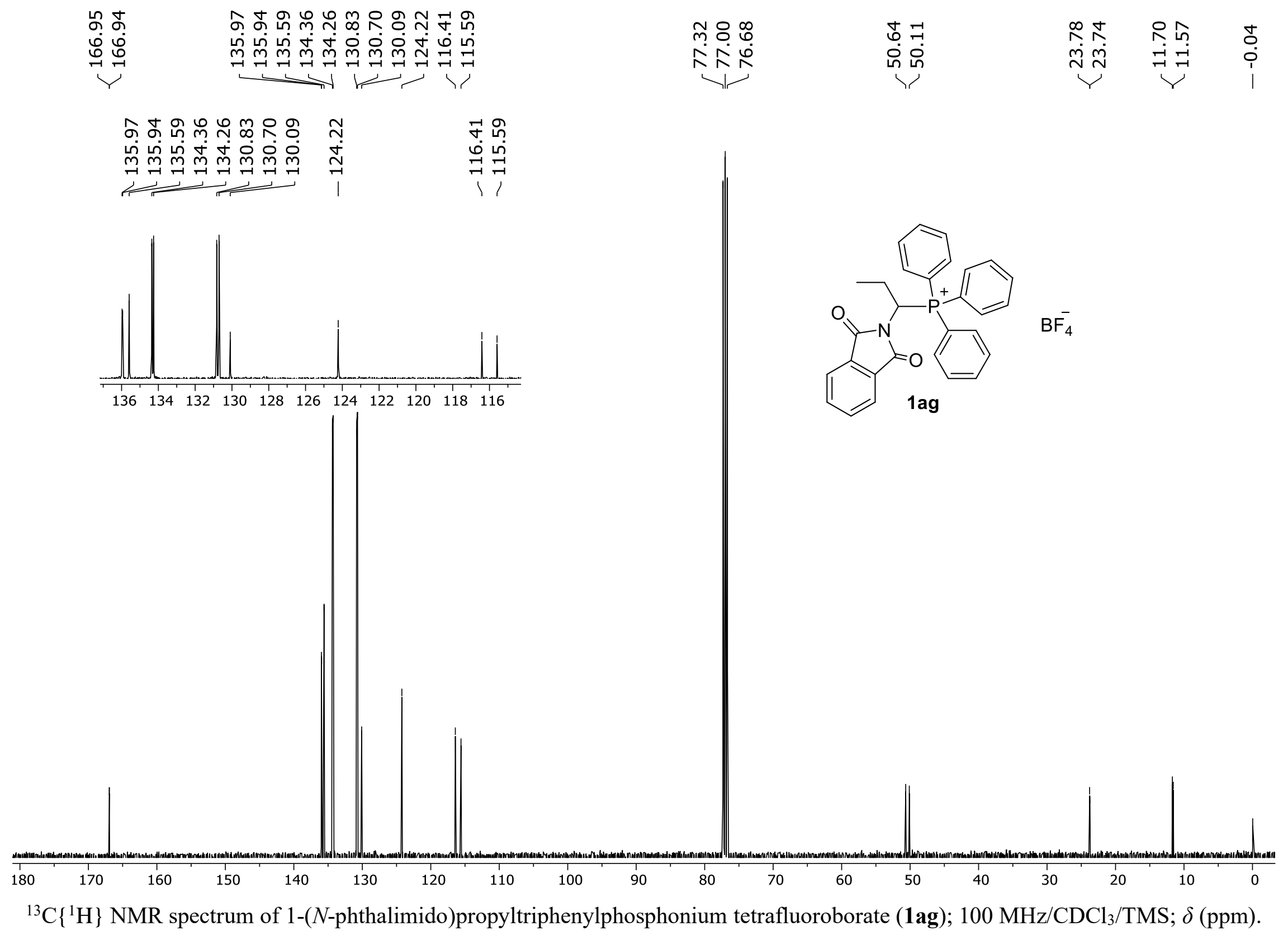




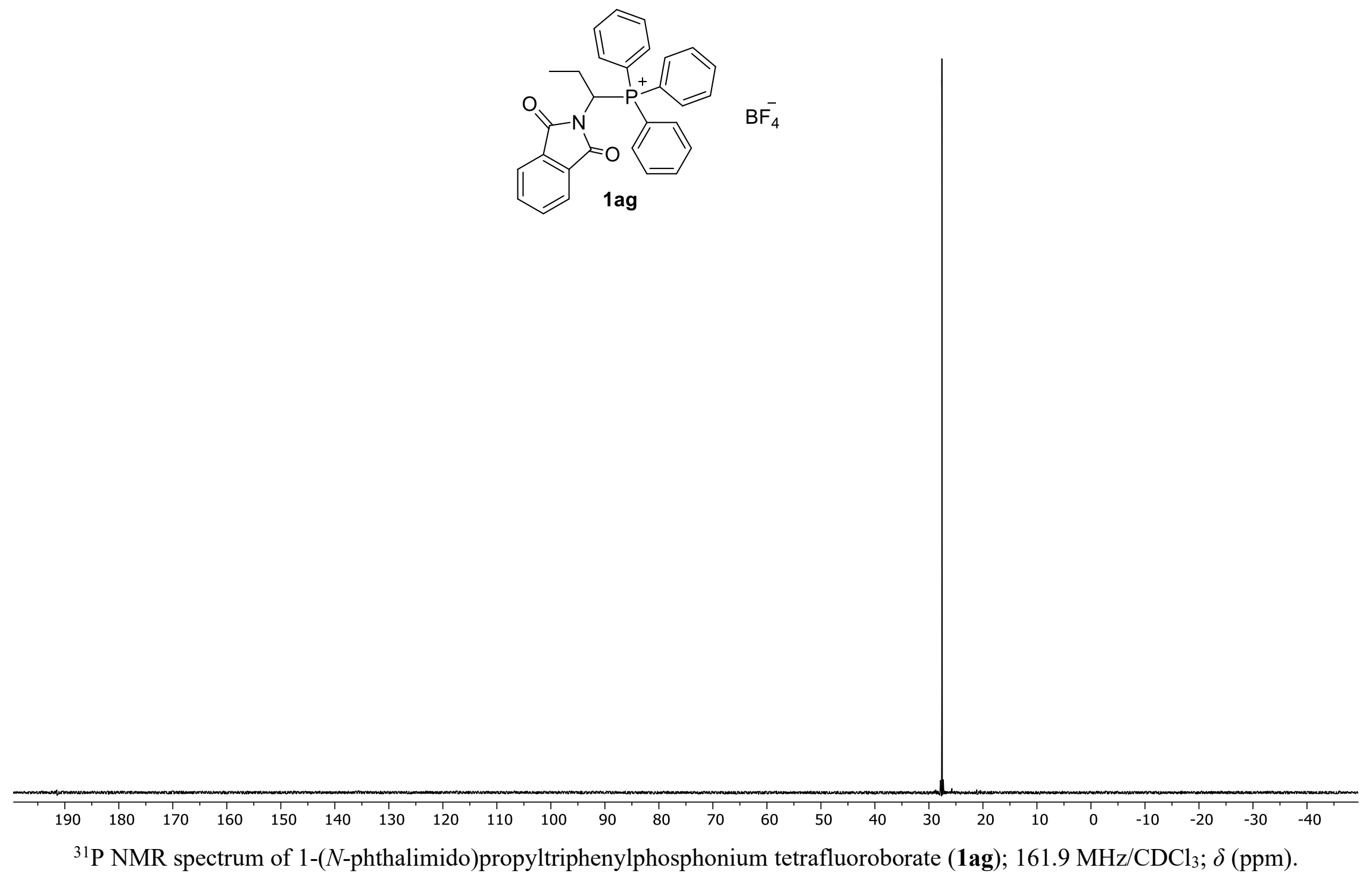




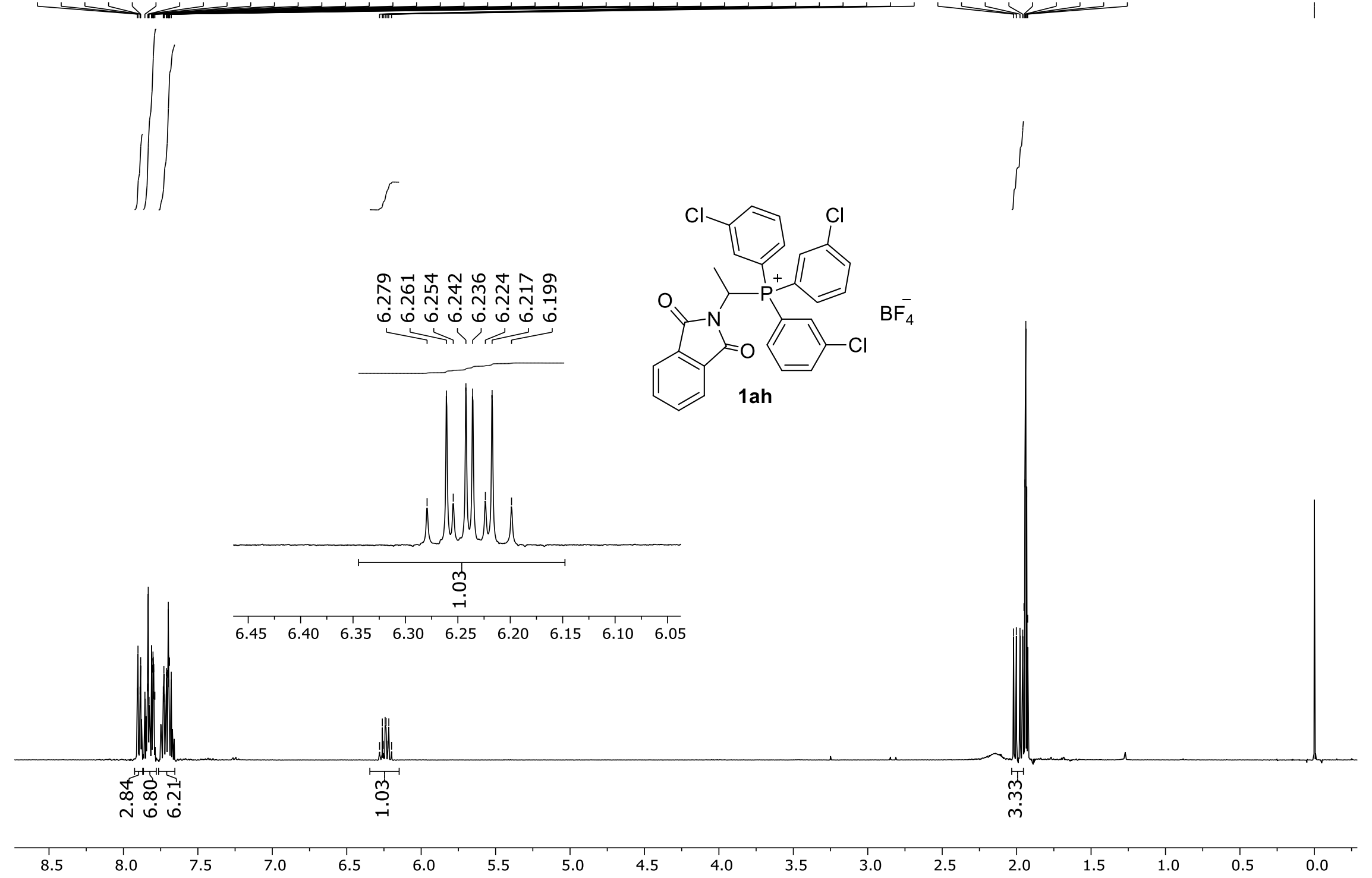

${ }^{1} \mathrm{H}$ NMR spectrum of 1-( $N$-phthalimido)ethyltris(3-chlorophenyl)phosphonium tetrafluoroborate (1ah); $400 \mathrm{MHz} / \mathrm{CD} 3 \mathrm{CN} / \mathrm{TMS} ; \delta$ (ppm). 

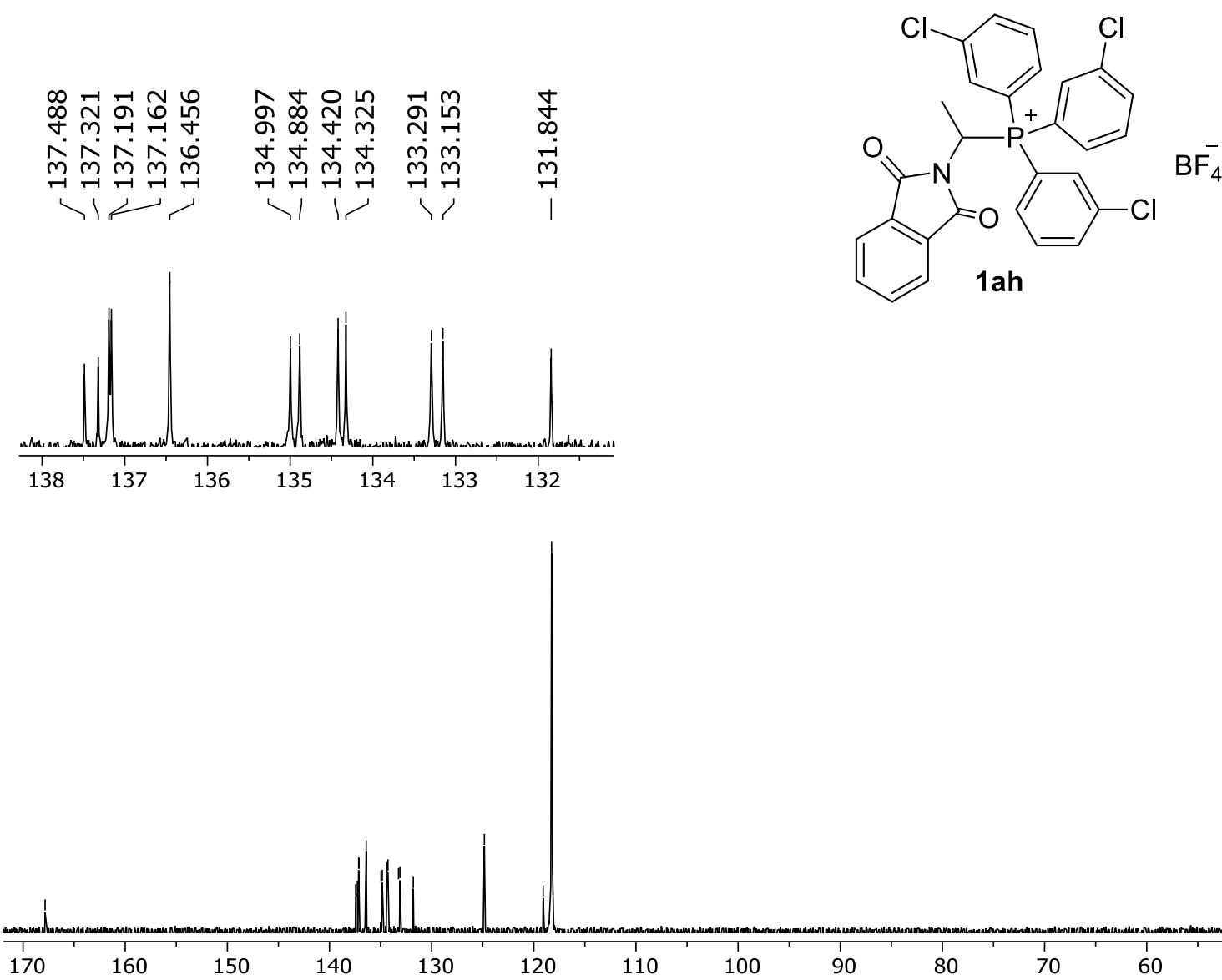

100

90

80
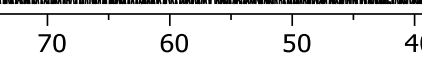

20

${ }^{13} \mathrm{C}\left\{{ }^{1} \mathrm{H}\right\}$ NMR spectrum of 1-( $N$-phthalimido)ethyltris(3-chlorophenyl)phosphonium tetrafluoroborate (1ah); $100 \mathrm{MHz} / \mathrm{CD}_{3} \mathrm{CN} / \mathrm{TMS} ; \delta$ (ppm). 

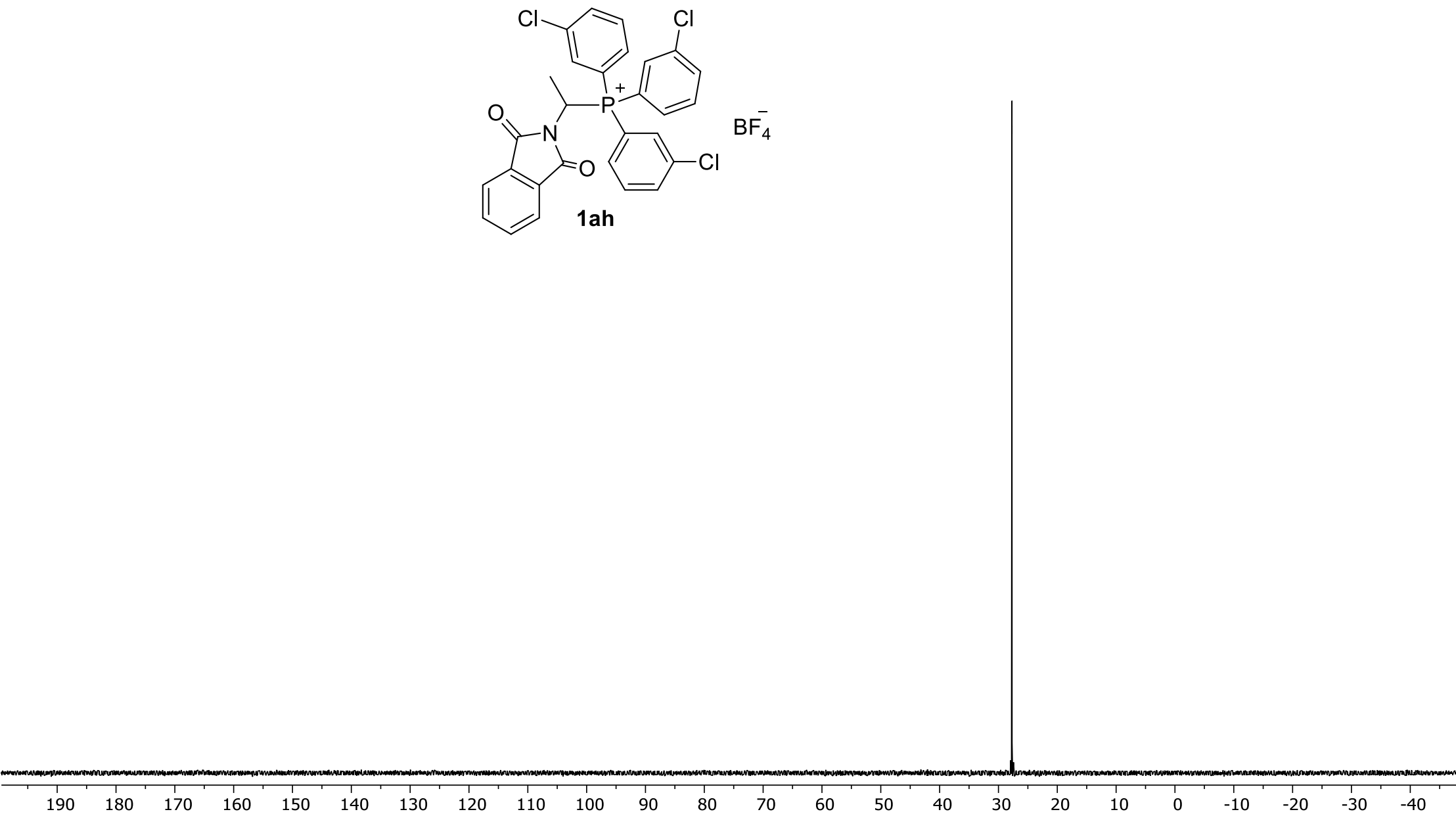

${ }^{31} \mathrm{P}$ NMR spectrum of 1-( $N$-phthalimido)ethyltris(3-chlorophenyl)phosphonium tetrafluoroborate (1ah); $161.9 \mathrm{MHz} / \mathrm{CD}{ }_{3} \mathrm{CN} ; \delta(\mathrm{ppm})$. 


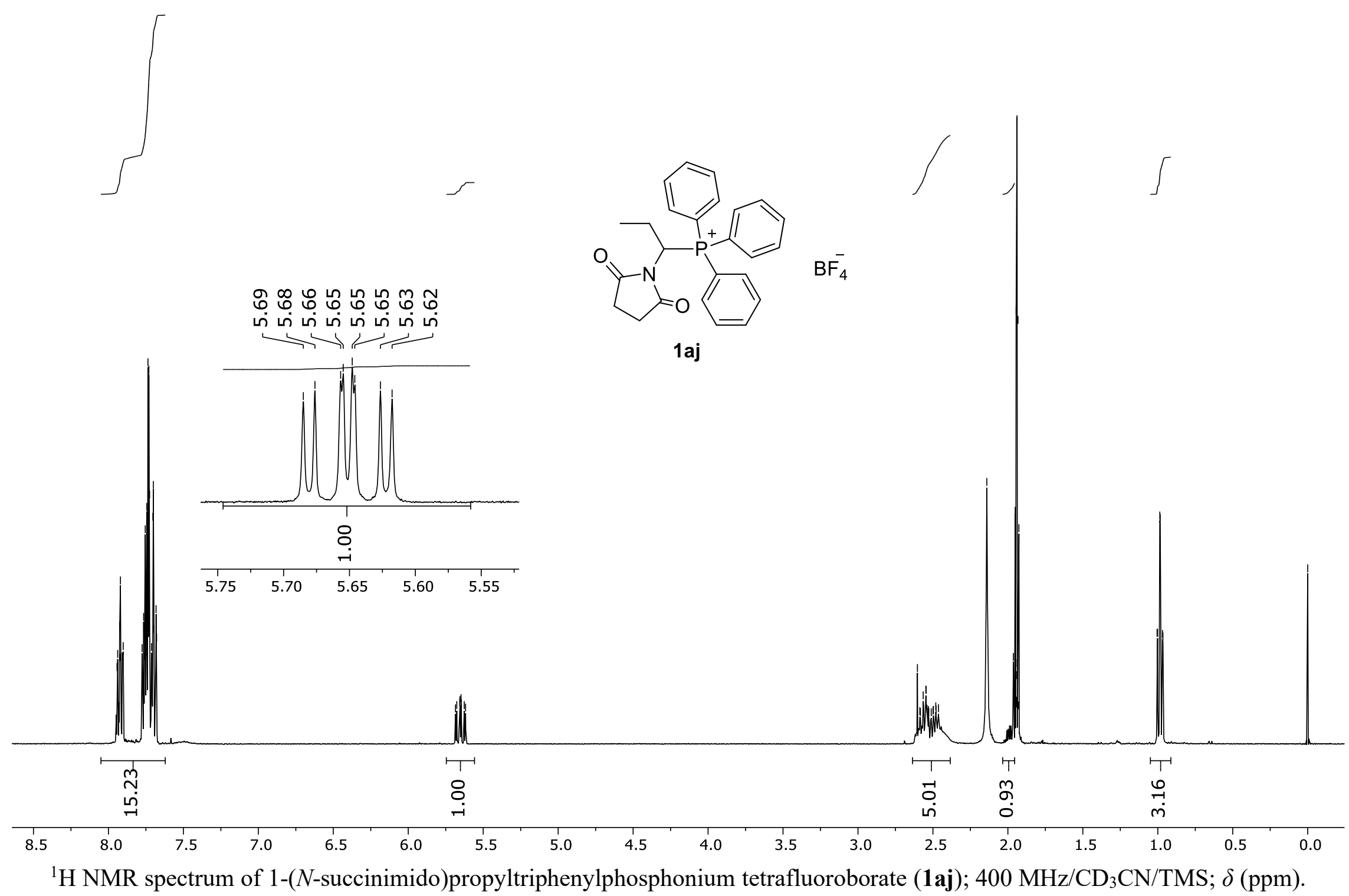




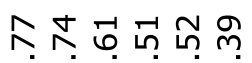

ம் ம்



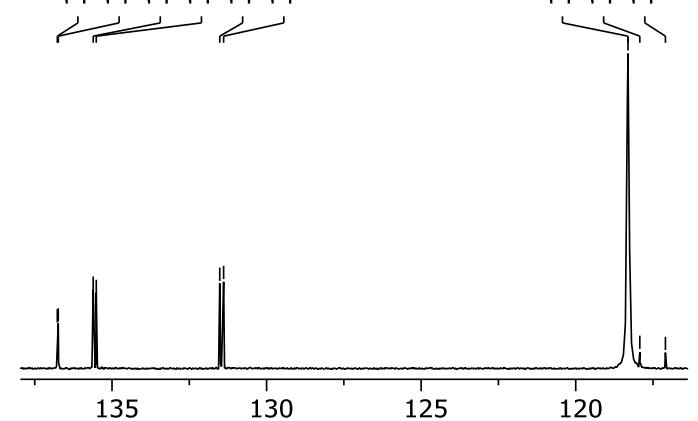

135

휘꾸유.․

学吉声

120
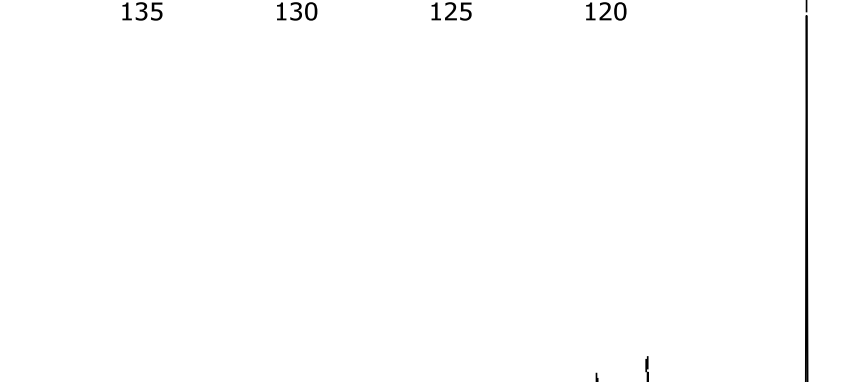

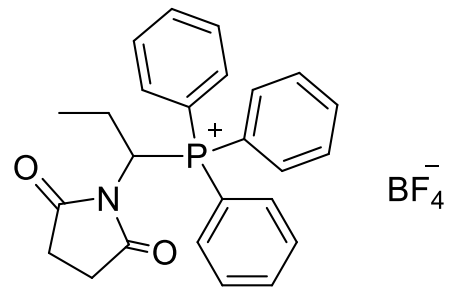

1aj

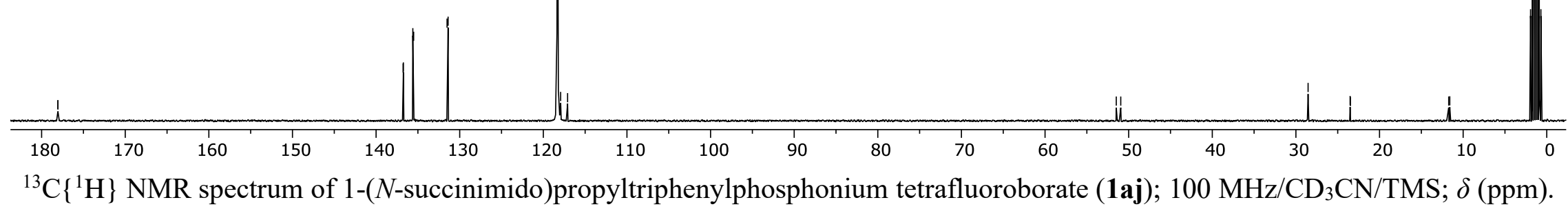




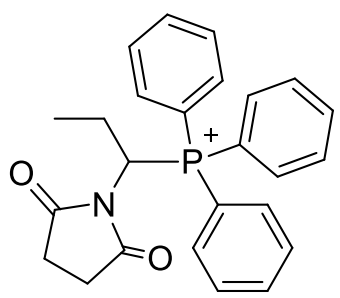

$\mathrm{BF}_{4}^{-}$

1aj

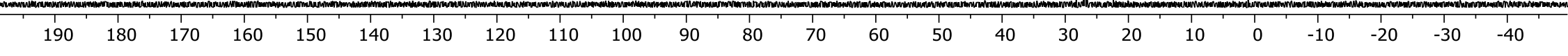

${ }^{31} \mathrm{P}$ NMR spectrum of 1-( $N$-succinimido)propyltriphenylphosphonium tetrafluoroborate (1aj); $161.9 \mathrm{MHz} / \mathrm{CD}{ }_{3} \mathrm{CN} ; \delta$ (ppm). 


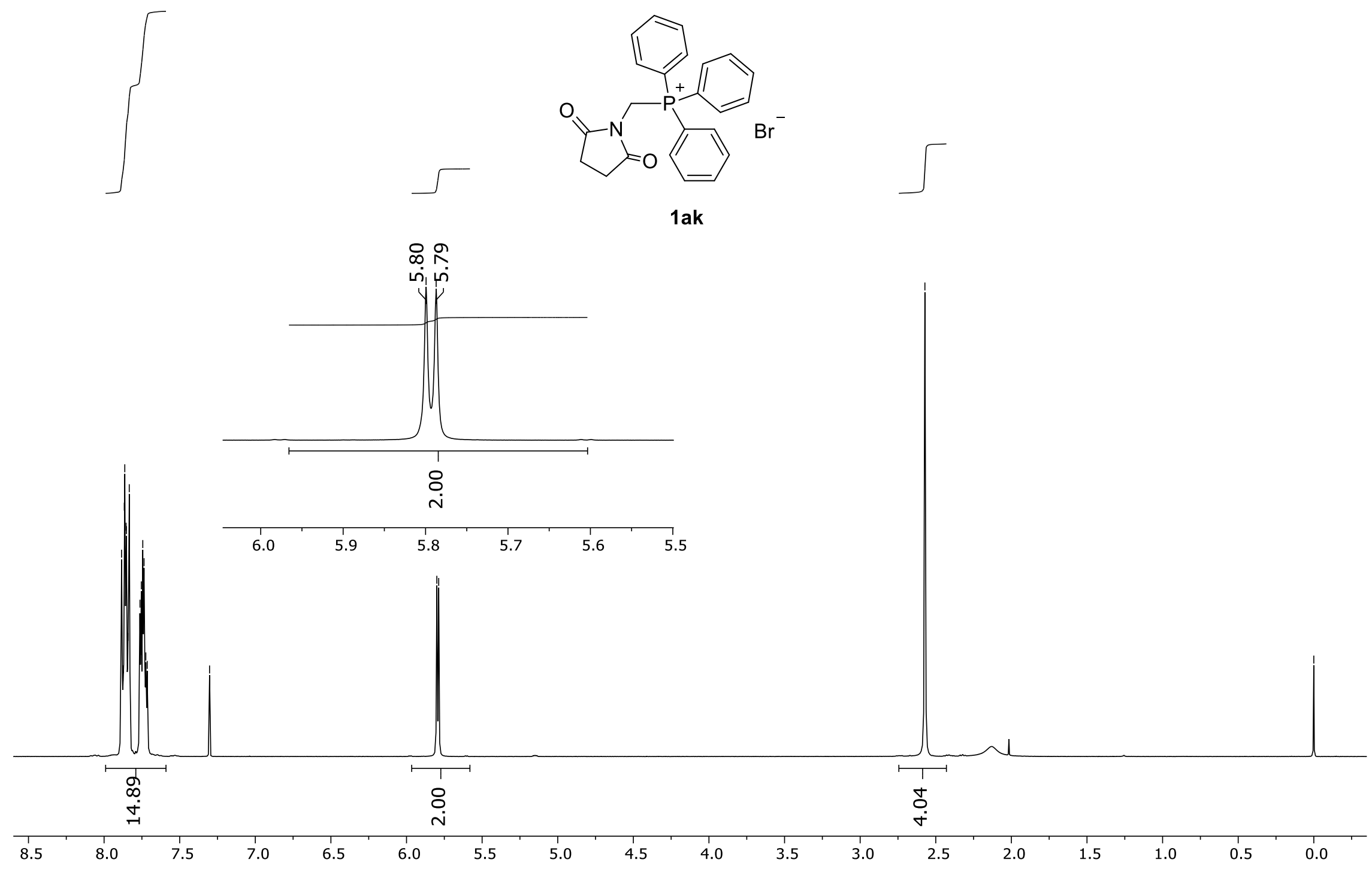

${ }^{1} \mathrm{H}$ NMR spectrum of 1-( $N$-succinimido)methyltriphenylphosphonium bromide (1ak); $400 \mathrm{MHz} / \mathrm{CDCl}_{3} / \mathrm{TMS} ; \delta$ (ppm). 


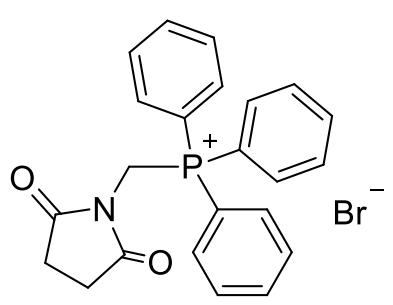

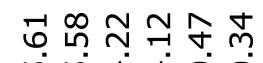

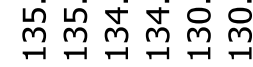

कo के

光

$\checkmark$

1

1 ak
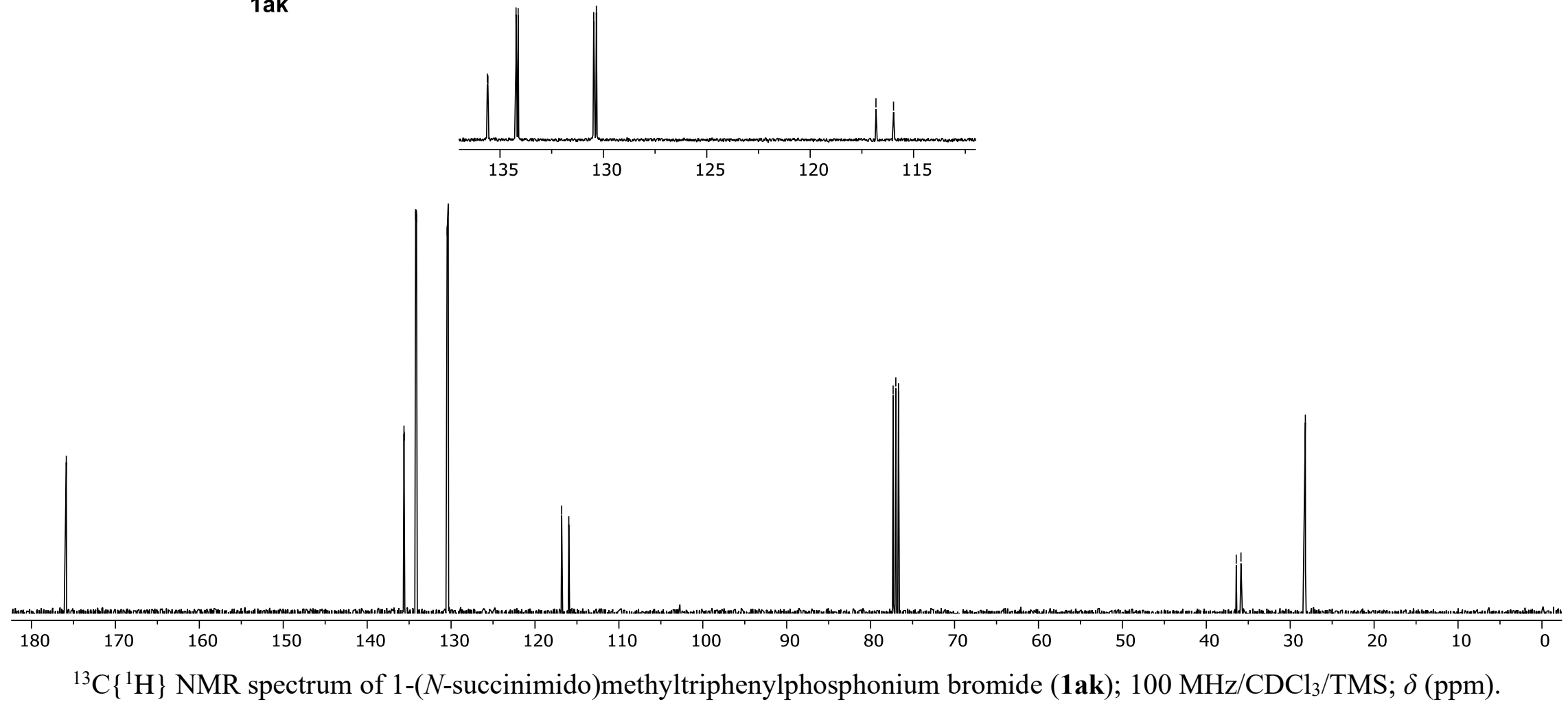


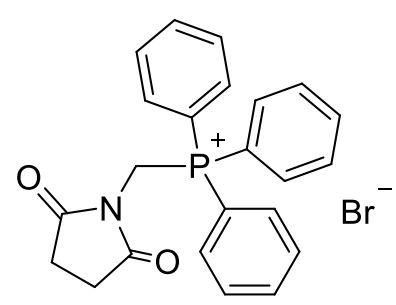

1 ak

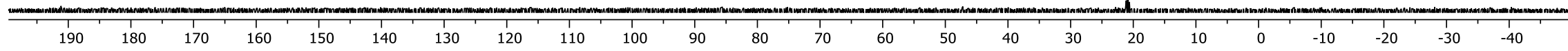

${ }^{31} \mathrm{P}$ NMR spectrum of 1 -( $N$-succinimido)methyltriphenylphosphonium bromide (1ak); $161.9 \mathrm{MHz} / \mathrm{CDCl}_{3} ; \delta$ (ppm). 


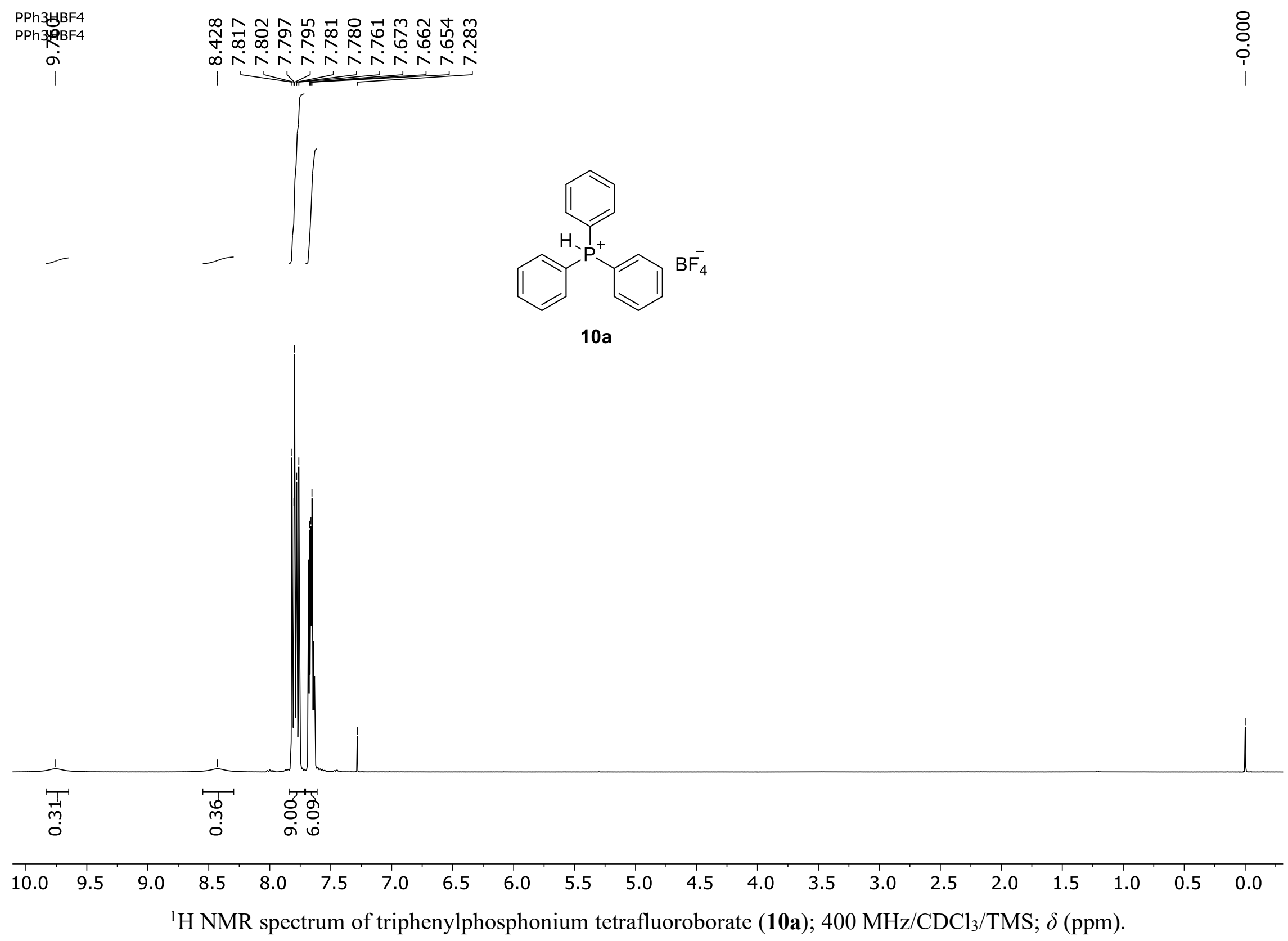




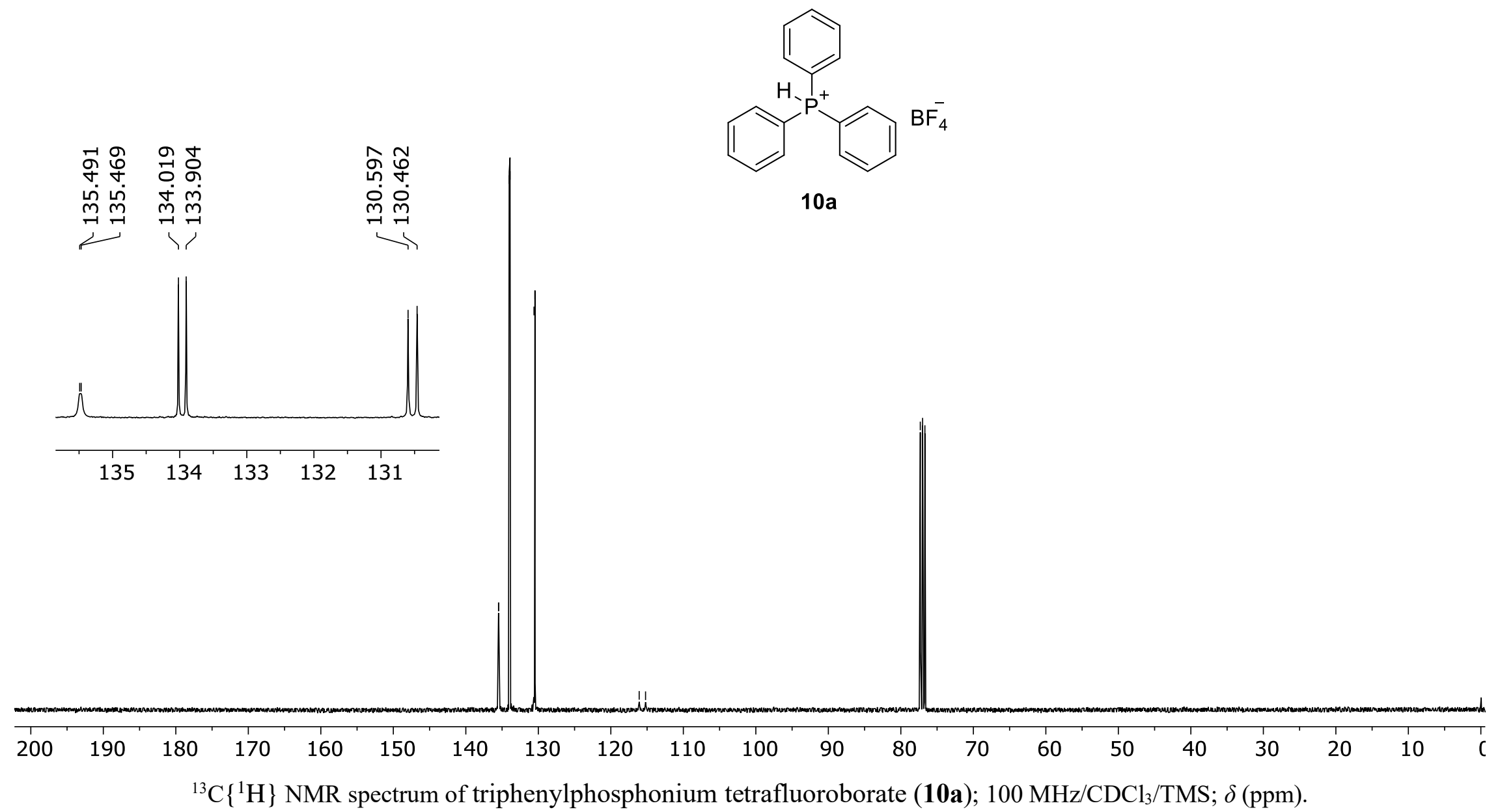




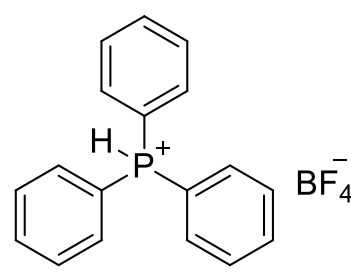

$10 a$

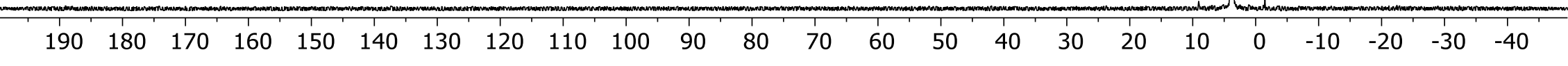

${ }^{31} \mathrm{P}$ NMR spectrum of triphenylphosphonium tetrafluoroborate (10a); $161.9 \mathrm{MHz} / \mathrm{CDCl}_{3} ; \delta(\mathrm{ppm})$. 

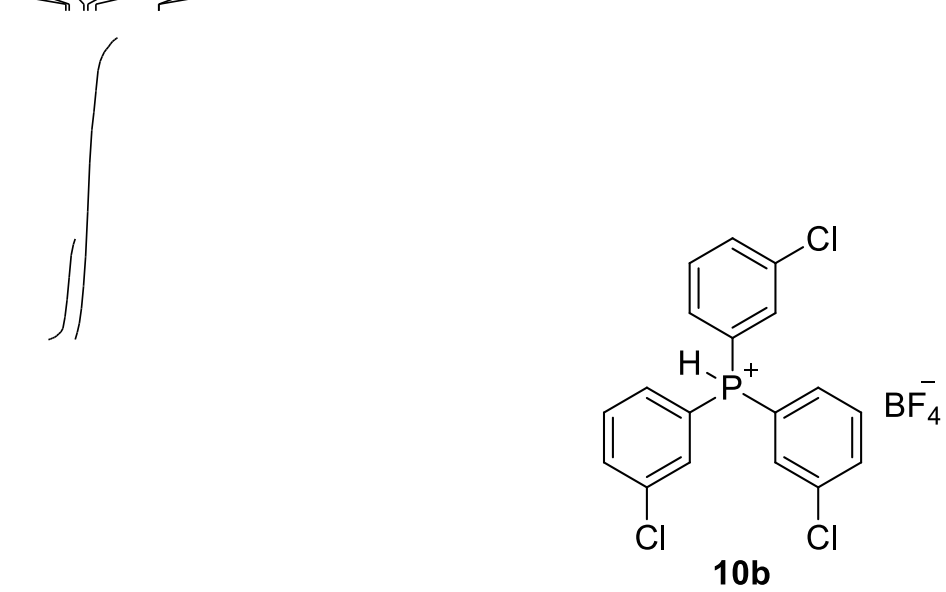

$10 \mathrm{~b}$
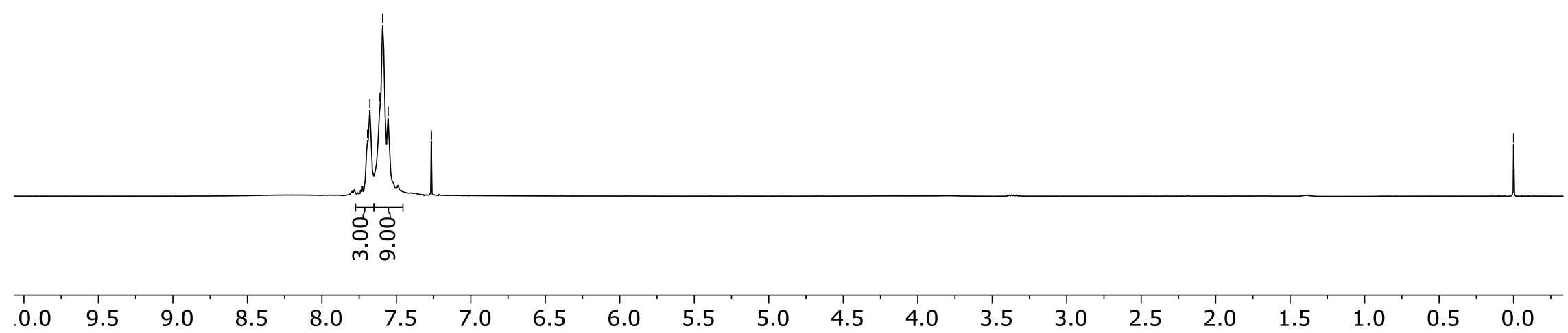

${ }^{1} \mathrm{H}$ NMR spectrum of tris(3-chlorophenyl)phosphonium tetrafluoroborate (10b); $400 \mathrm{MHz} / \mathrm{CDCl}_{3} / \mathrm{TMS} ; \delta$ (ppm). 


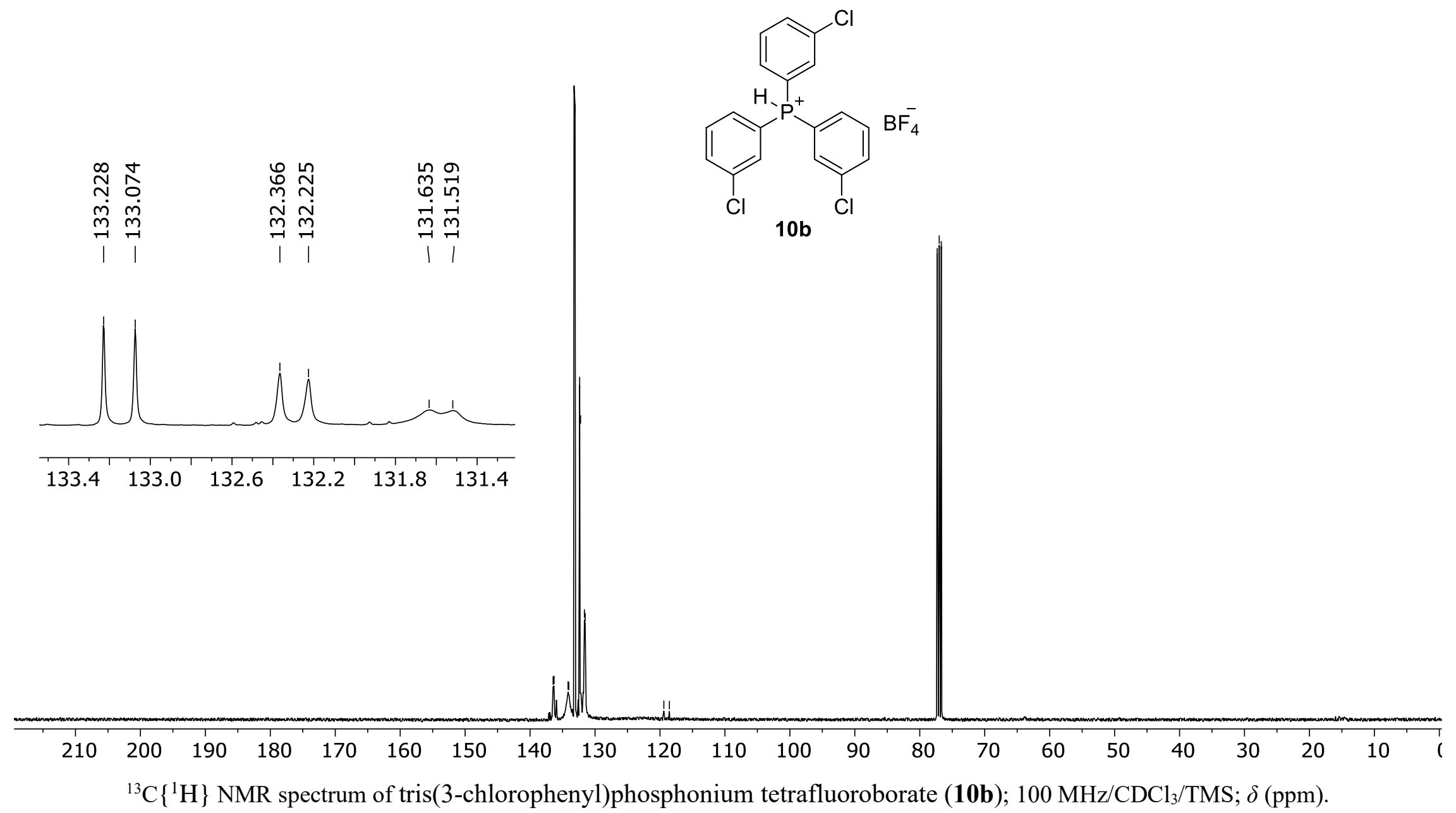



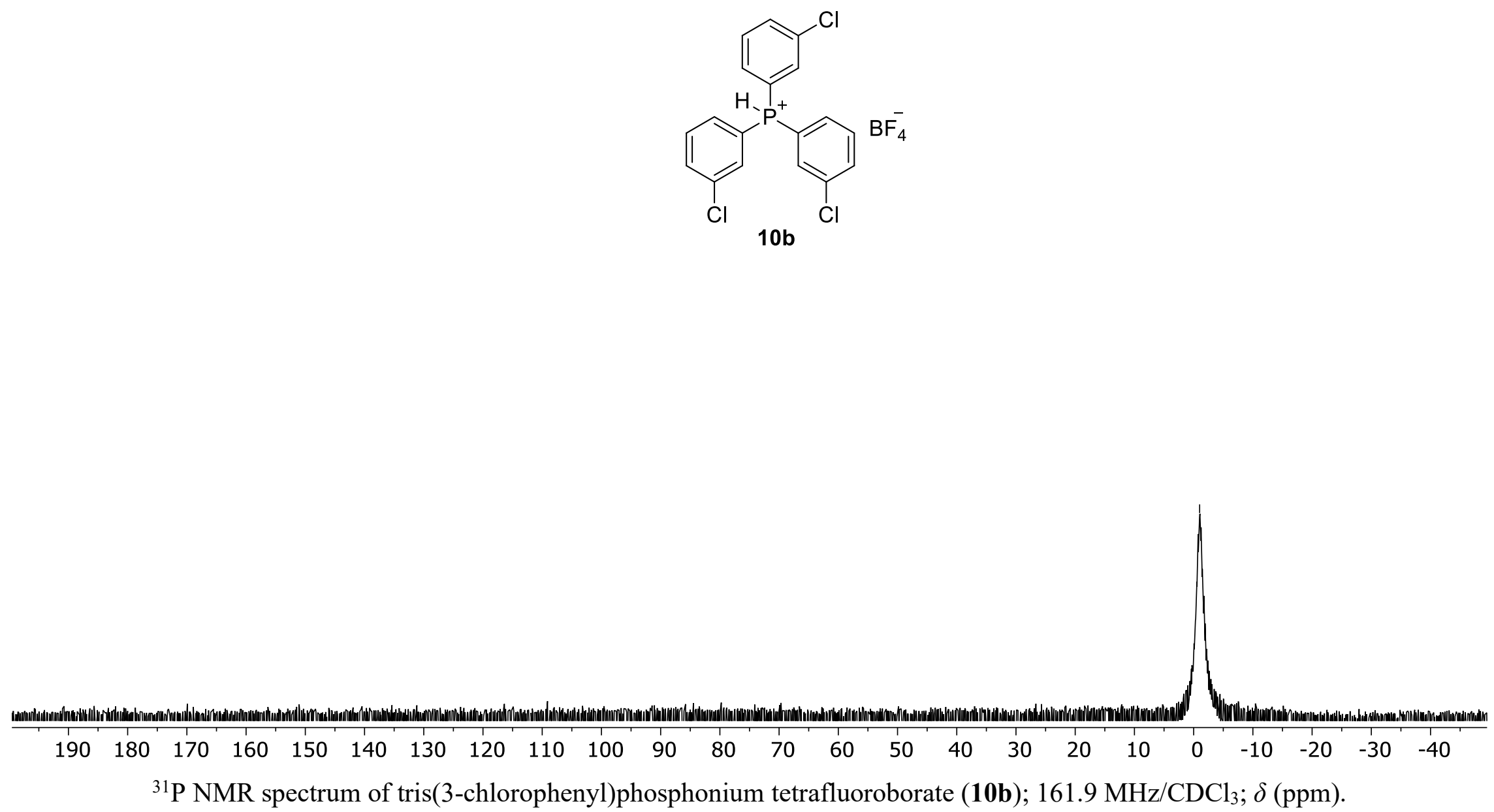


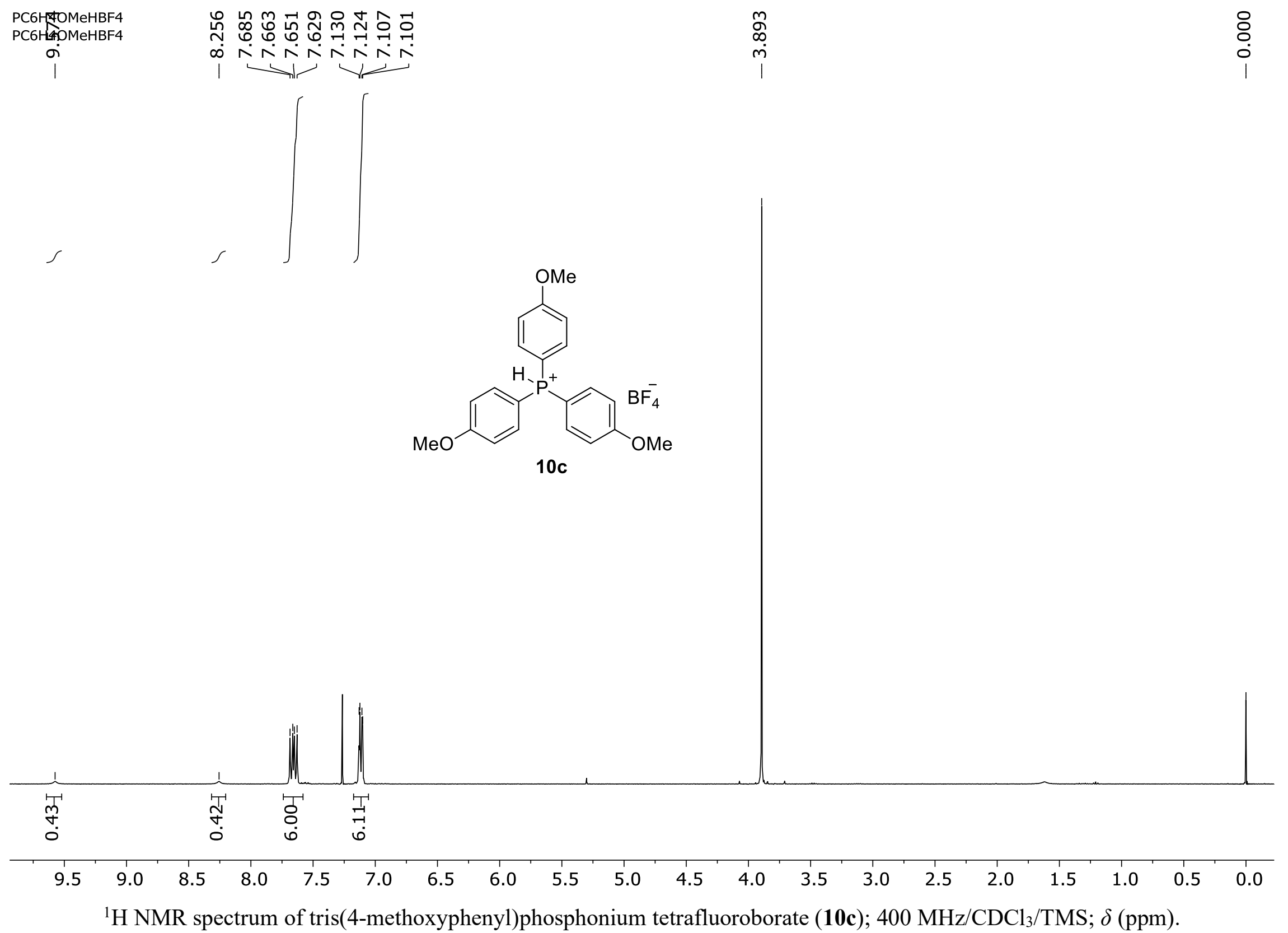




\begin{tabular}{|c|c|c|}
\hline 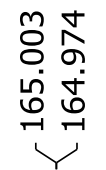 & 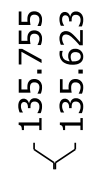 & 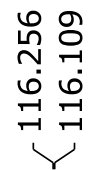 \\
\hline
\end{tabular}
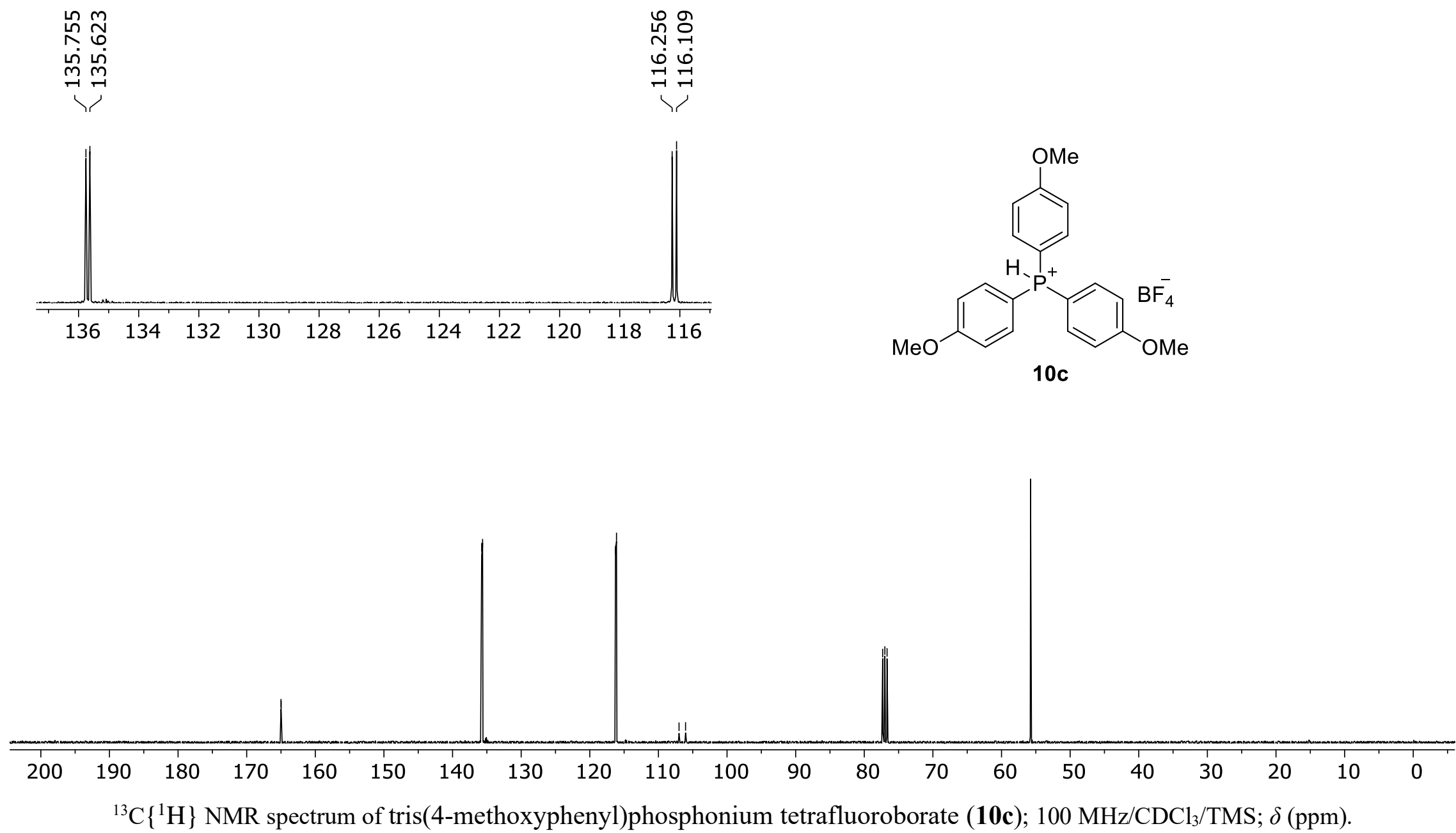

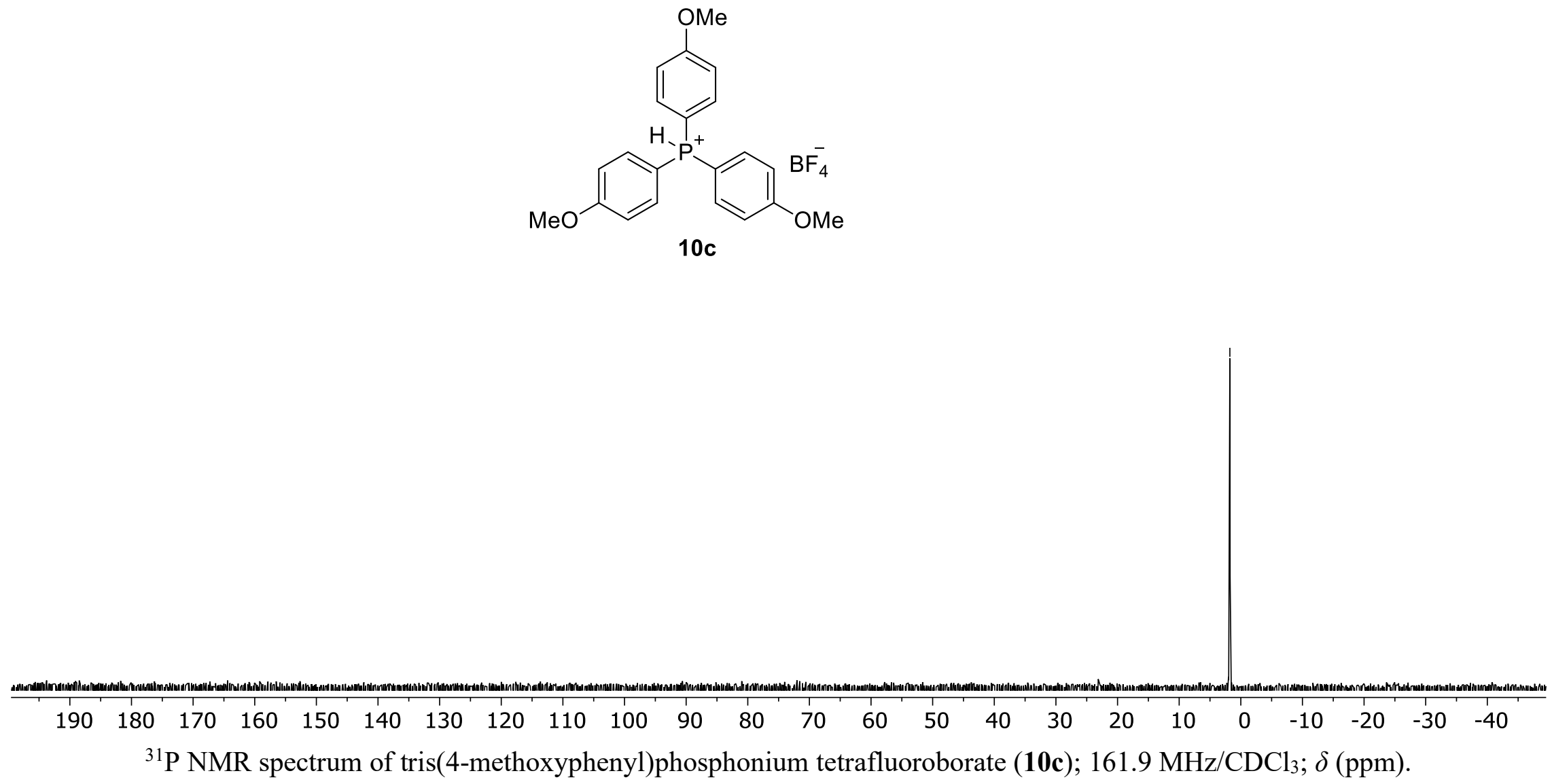

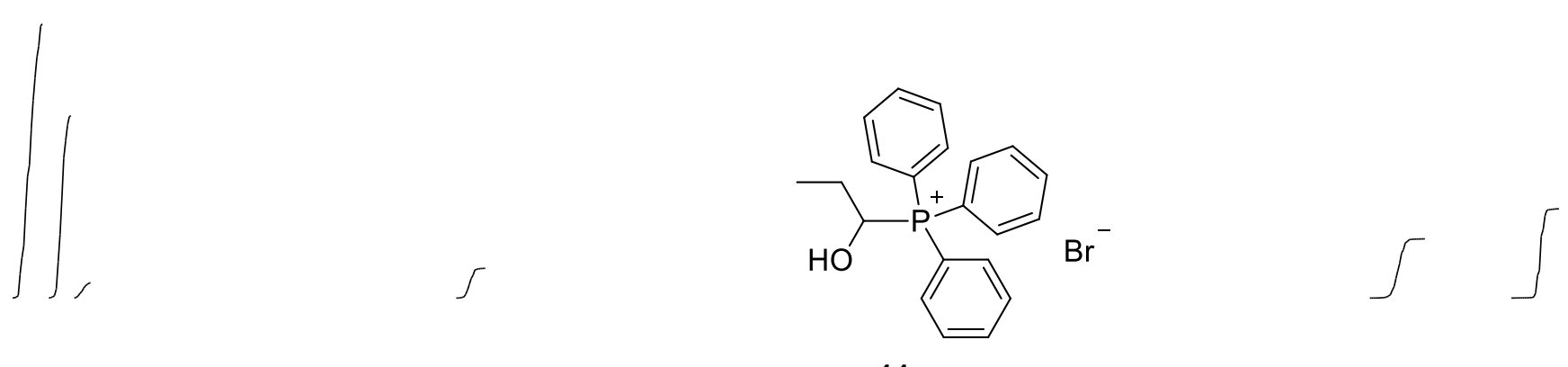

$11 \mathrm{a}$

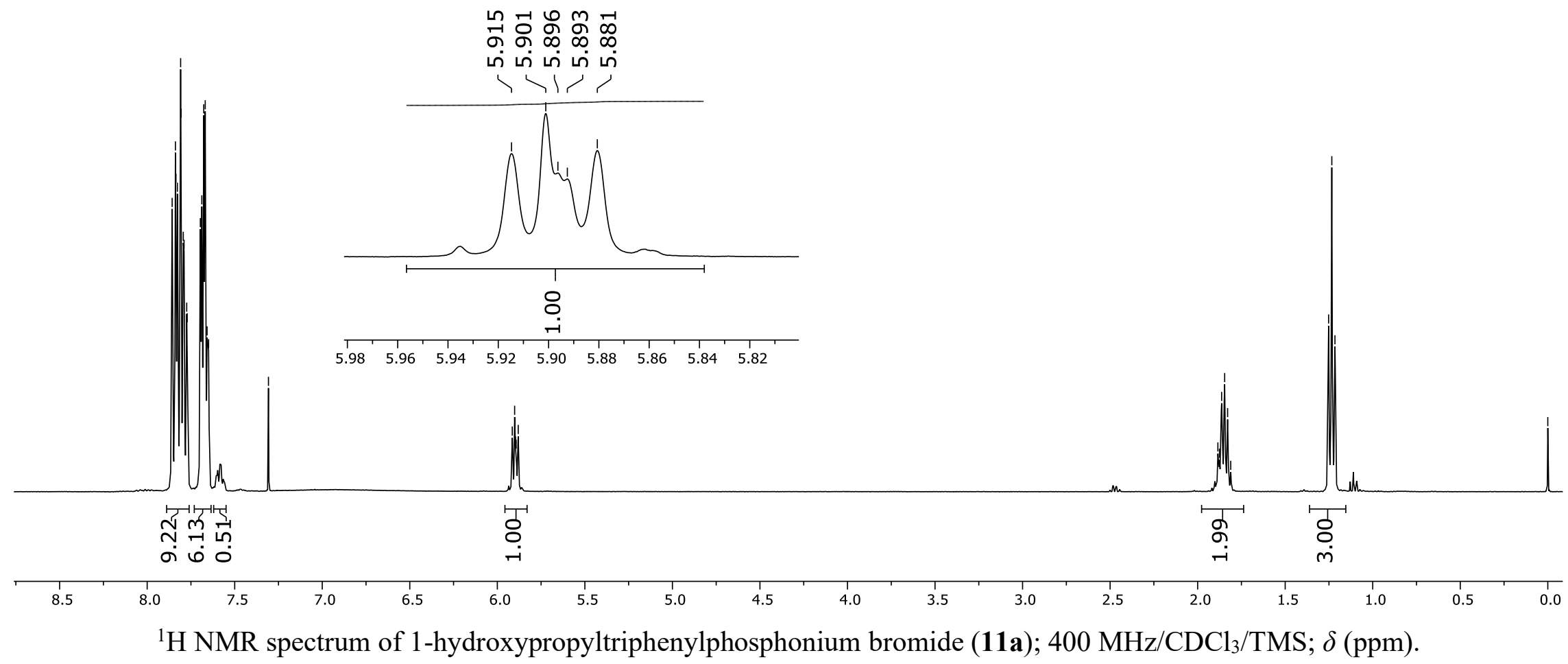




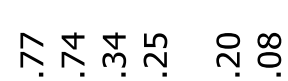

状岕离离

윰

学

प

각
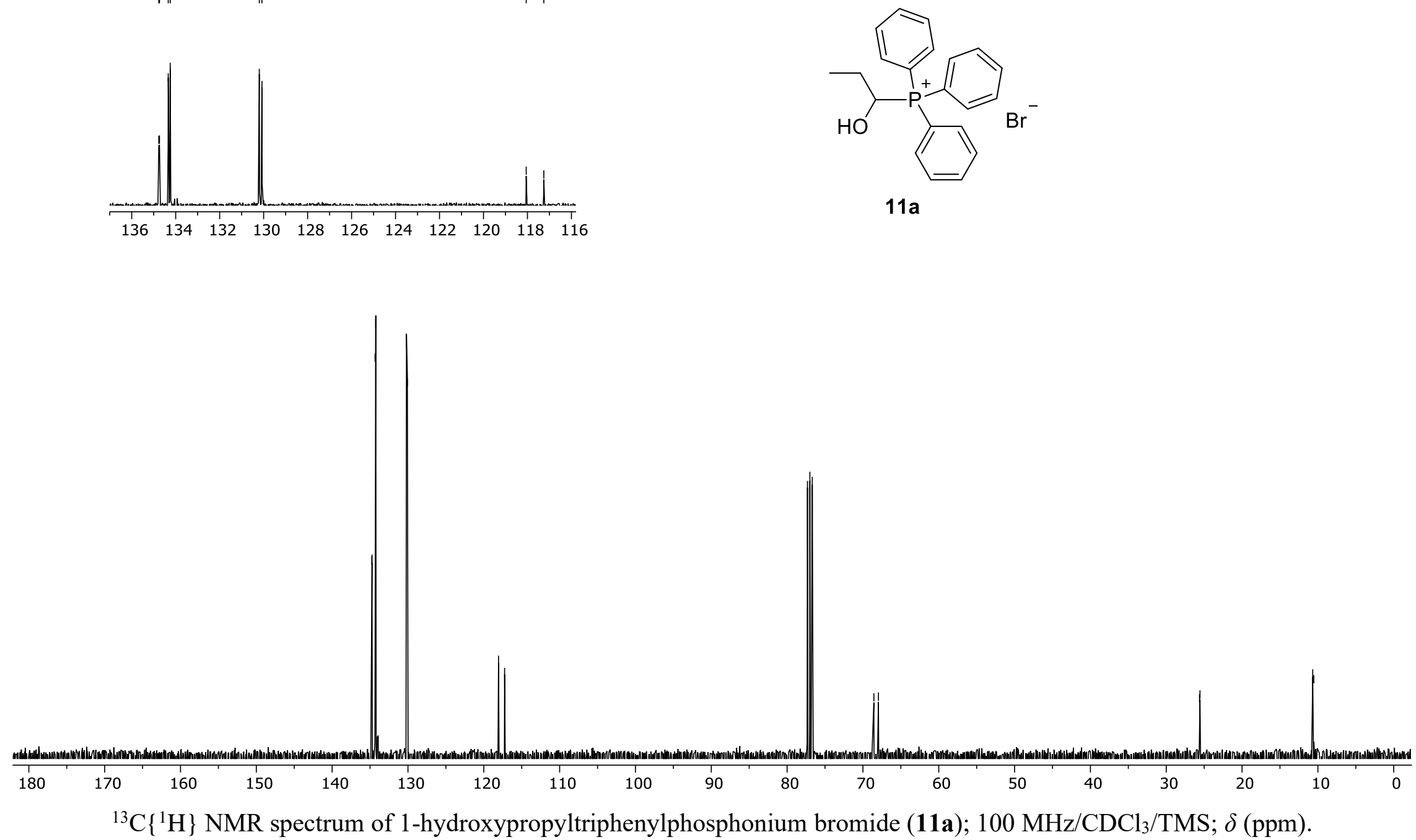


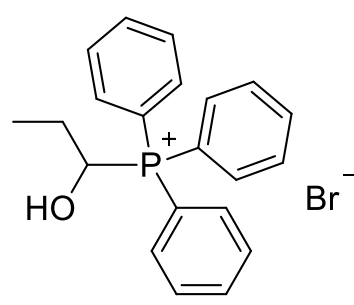

11a

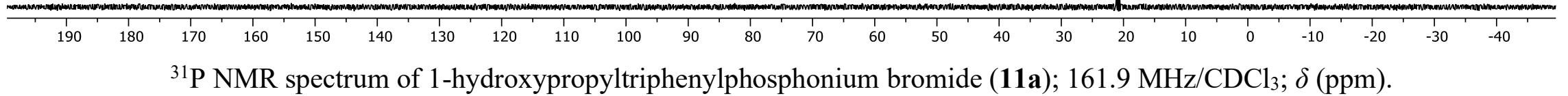




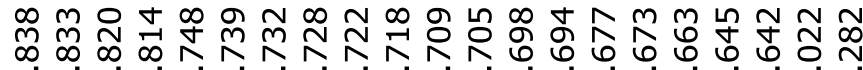

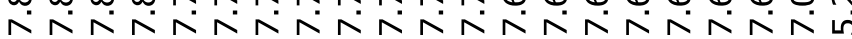

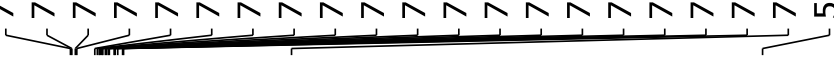

$\int$
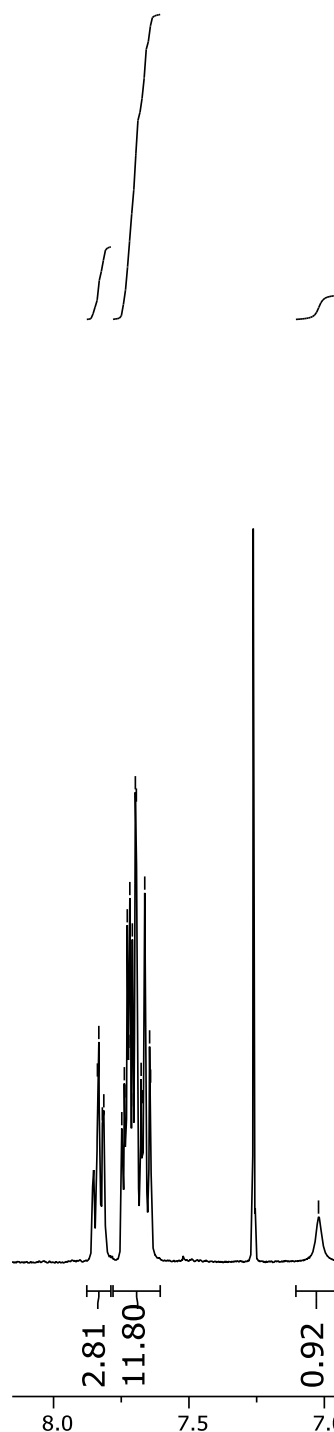

7.5

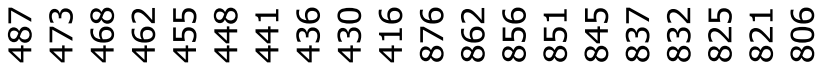

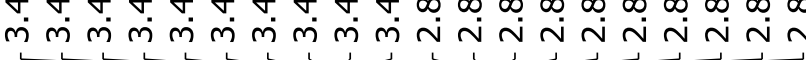

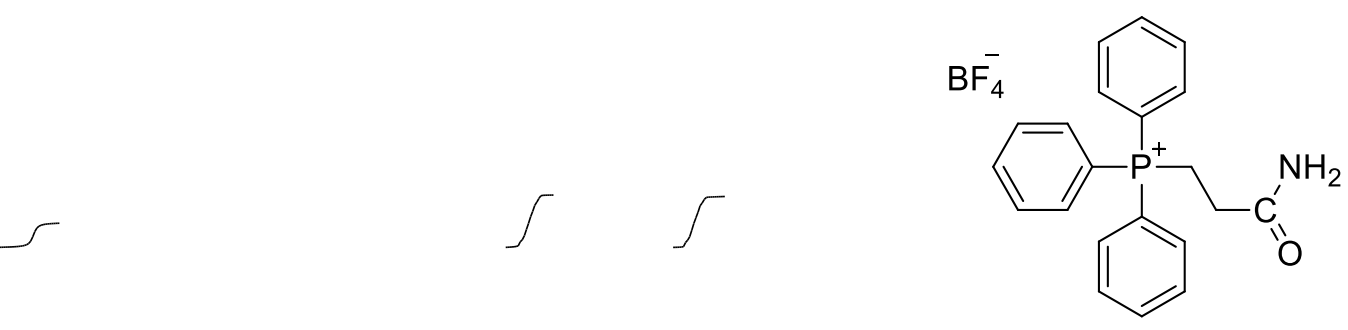

14

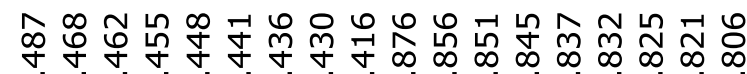

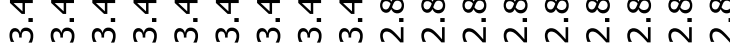

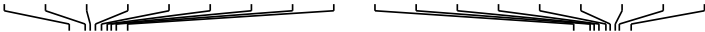
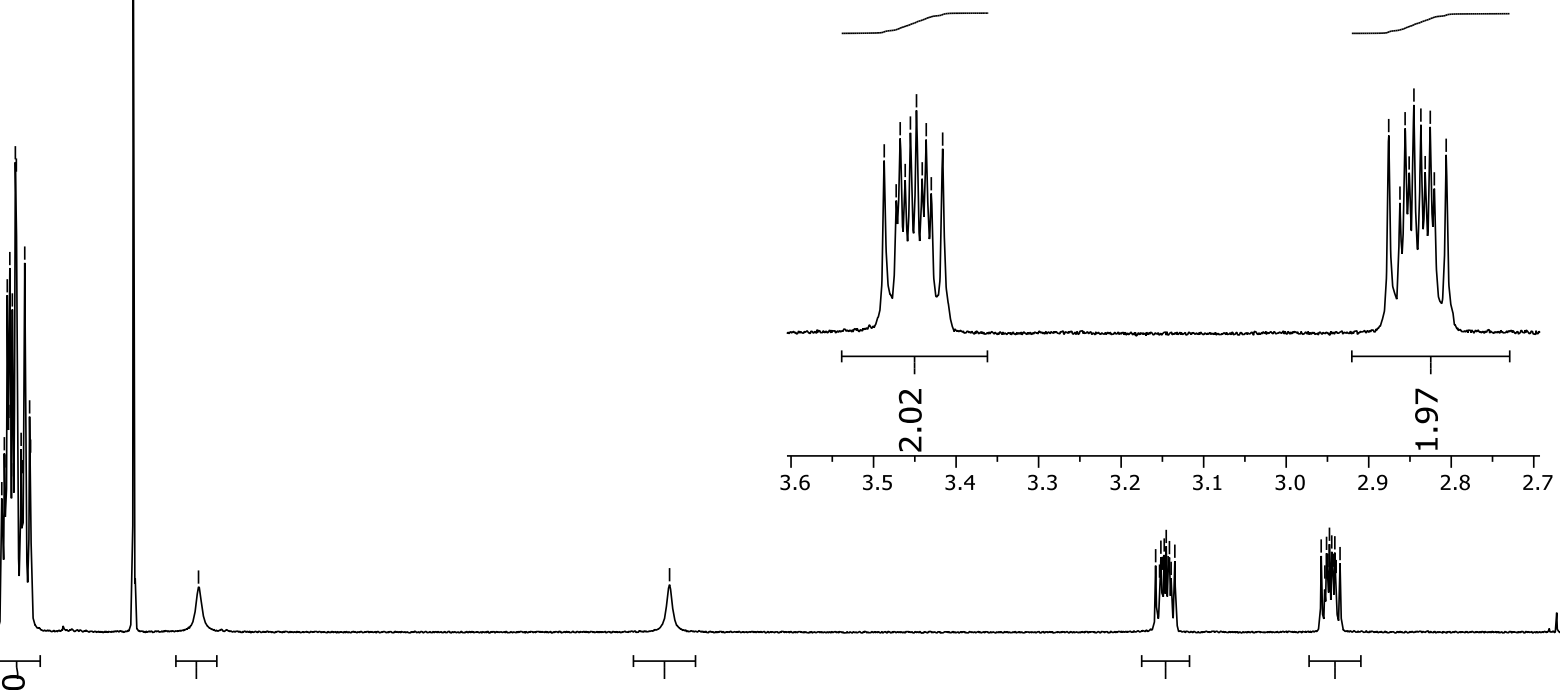

1

5.0

4.5

4.0

$3.5 \quad 3.0$

2.5

2.0

1.5

1.0

H NMR spectrum of 2-carbamoyletyhyltriphenylphosphonium tetrafluoroborate (14); $400 \mathrm{MHz} / \mathrm{CDCl}_{3} / \mathrm{TMS} ; \delta$ (ppm). 


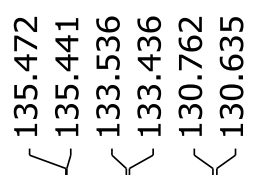

ํํํํํㅇ

극도

$m \underset{\rightarrow}{m} \stackrel{m}{\rightarrow} m m$

각
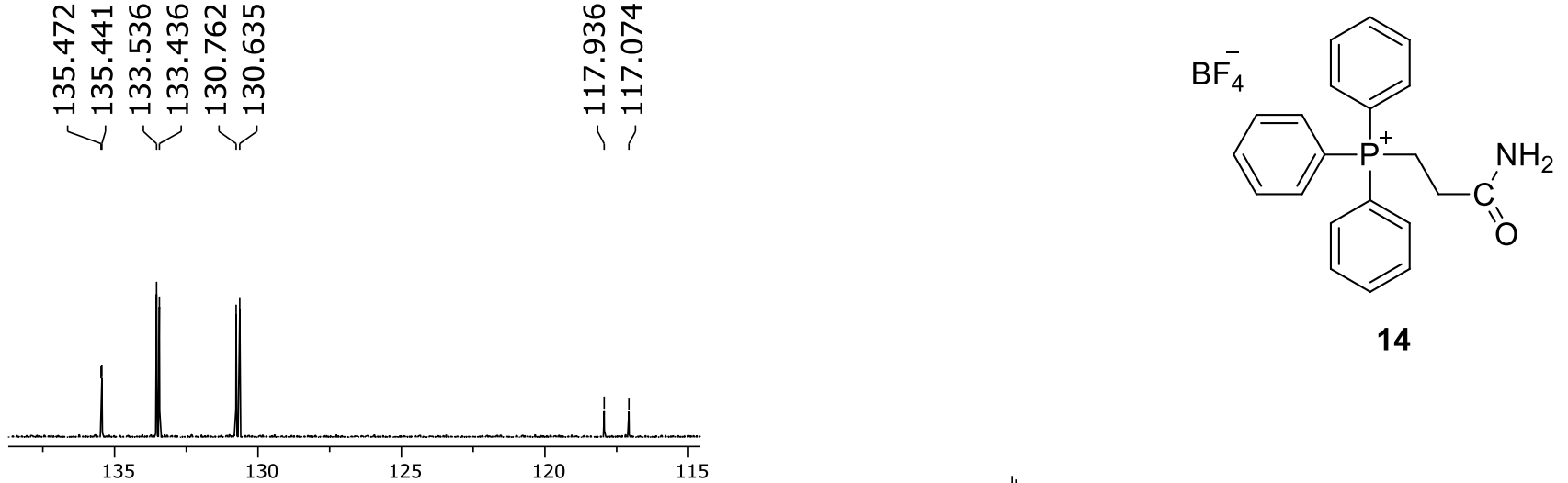

14

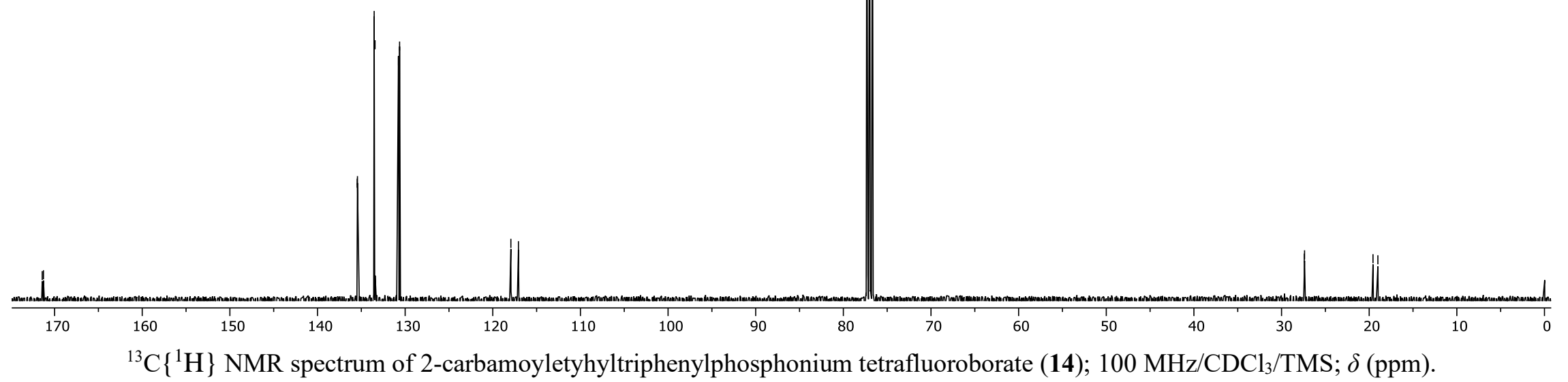



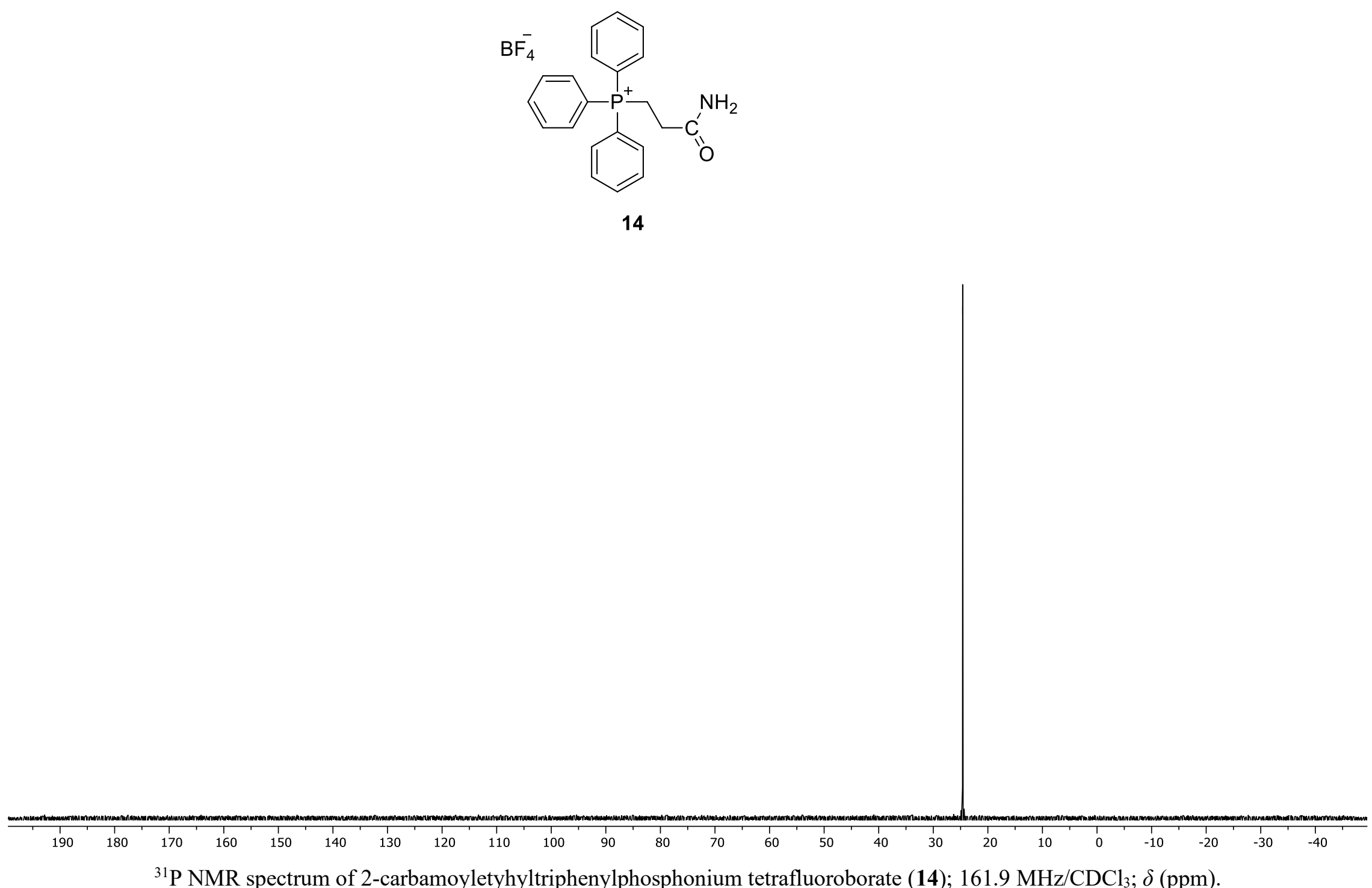


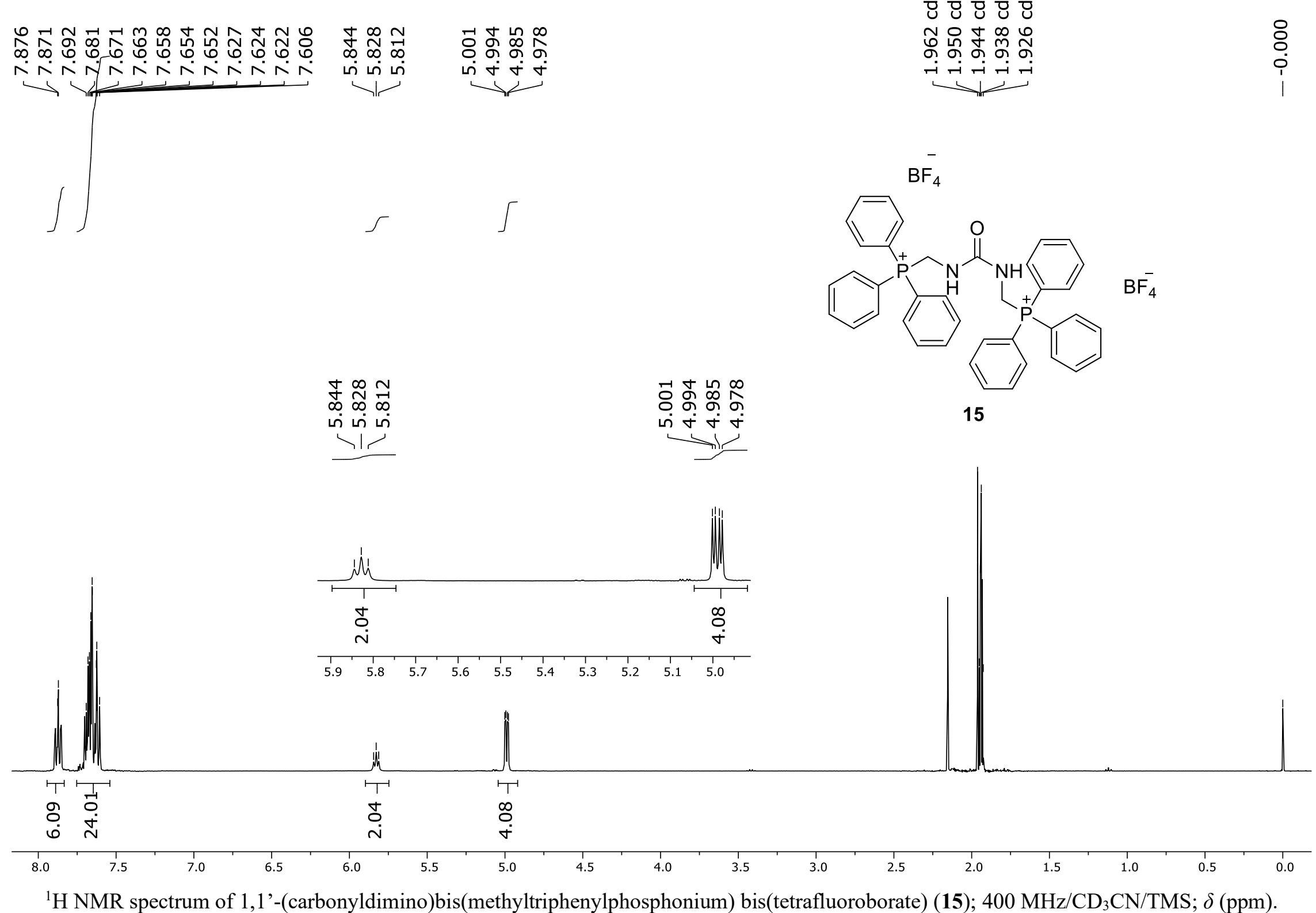




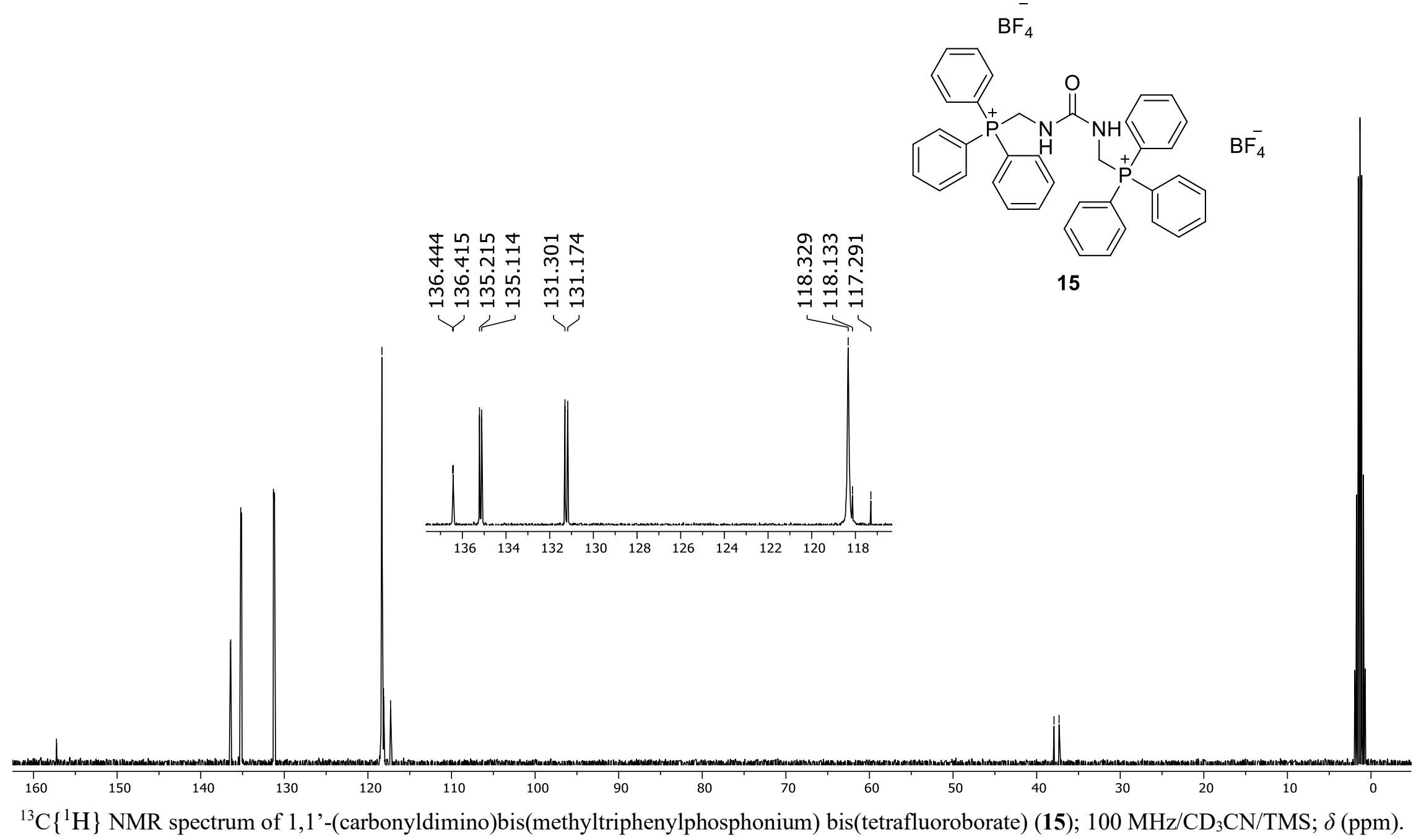




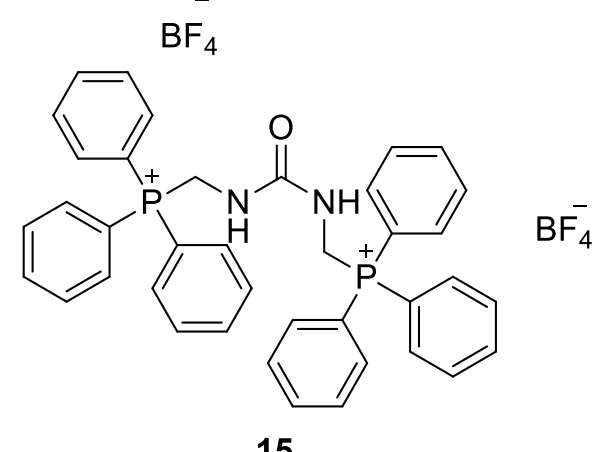

15

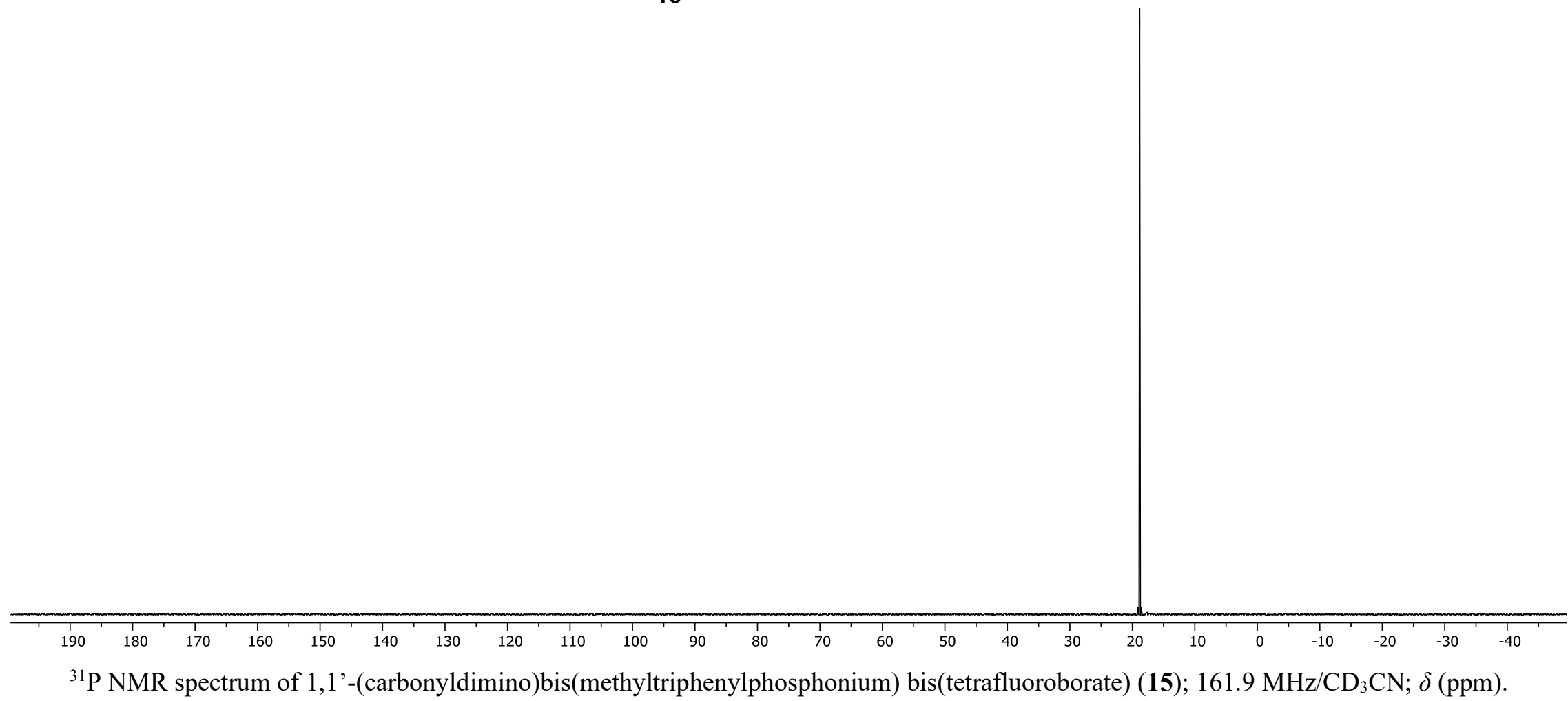




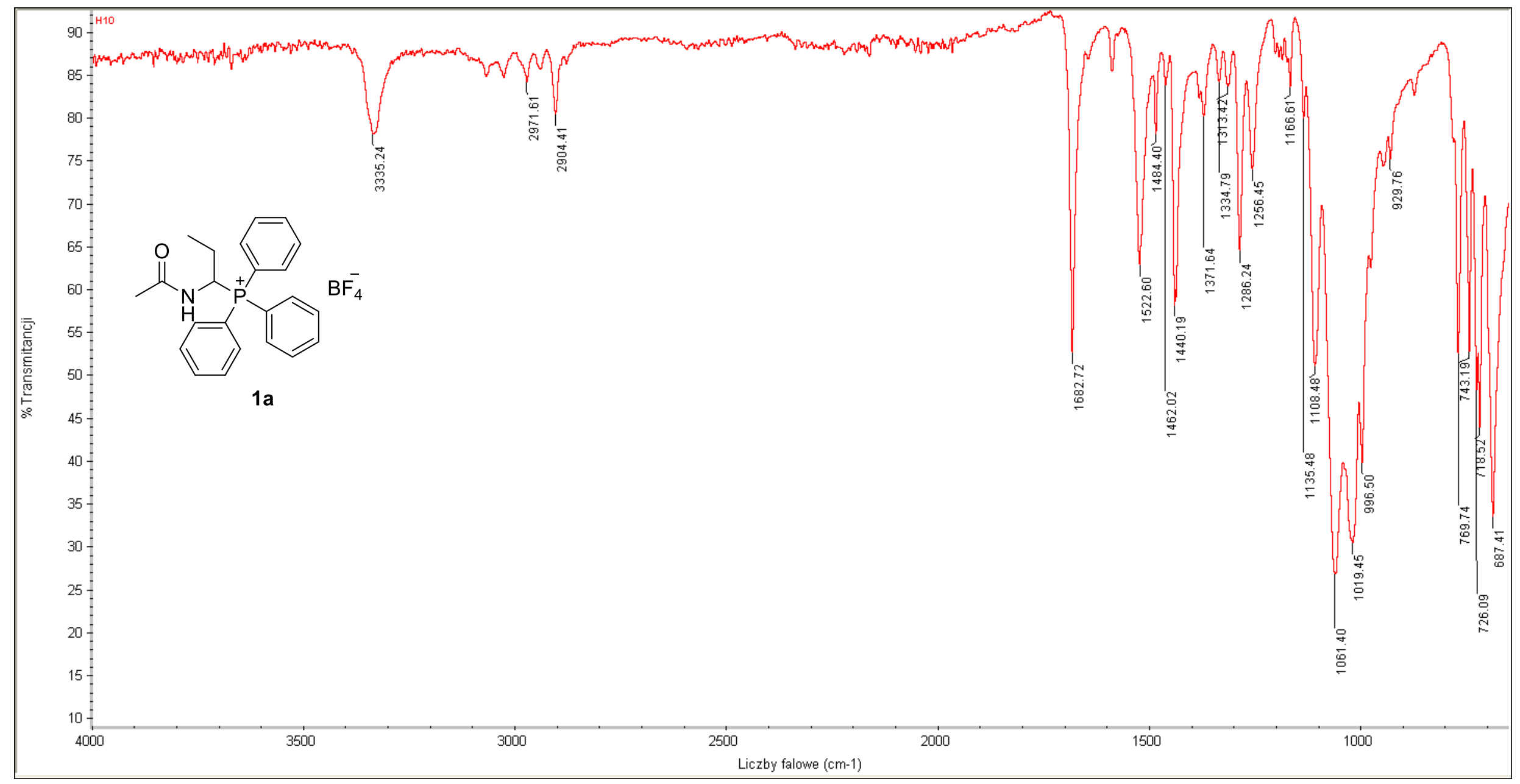

IR spectrum of 1-(N-acetylamino)propyltriphenylphosphonium tetrafluoroborate (1a); ATR $\left(\mathrm{cm}^{-1}\right)$. 


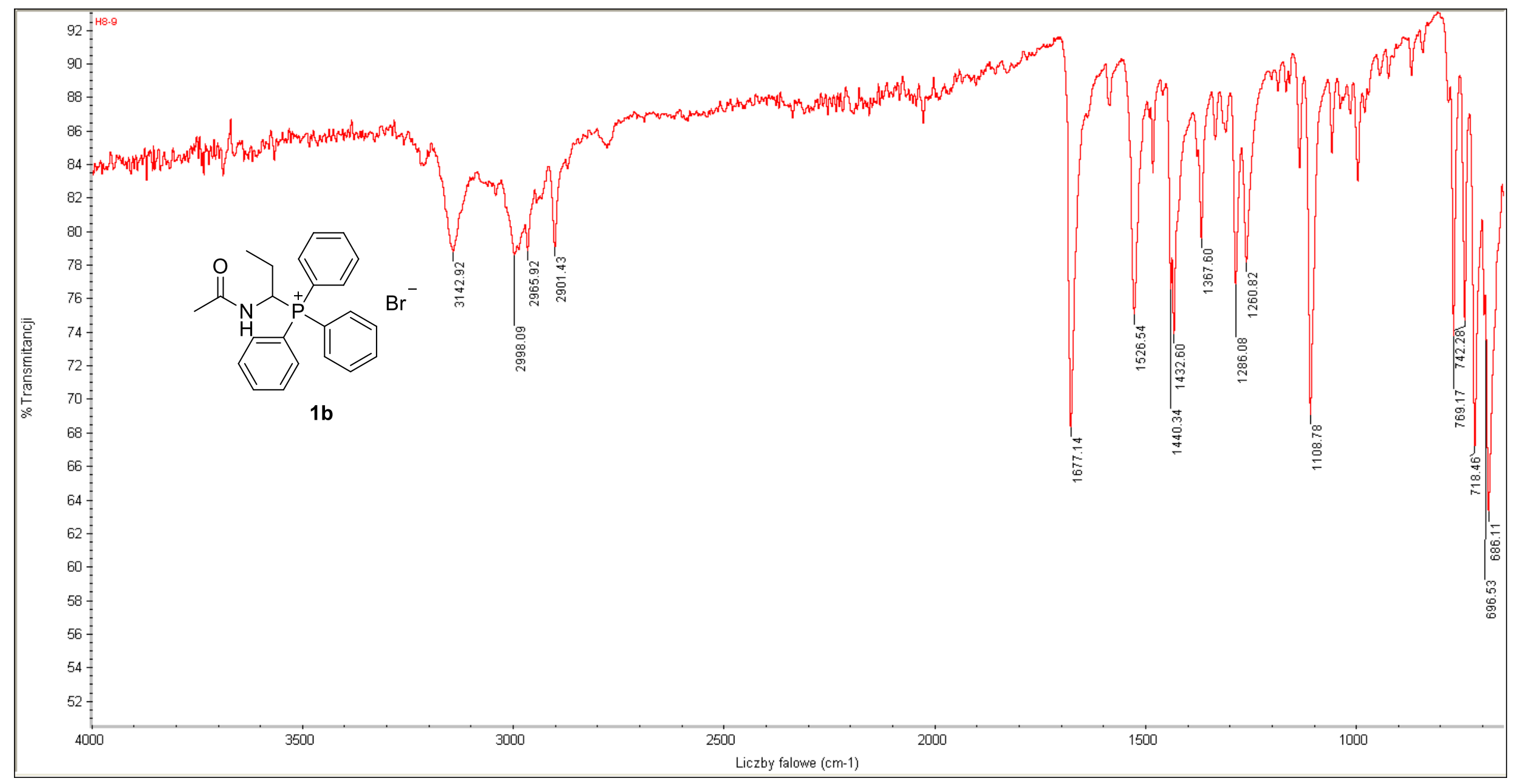

IR spectrum of 1-(N-acetylamino)propyltriphenylphosphonium bromide (1b); ATR $\left(\mathrm{cm}^{-1}\right)$. 


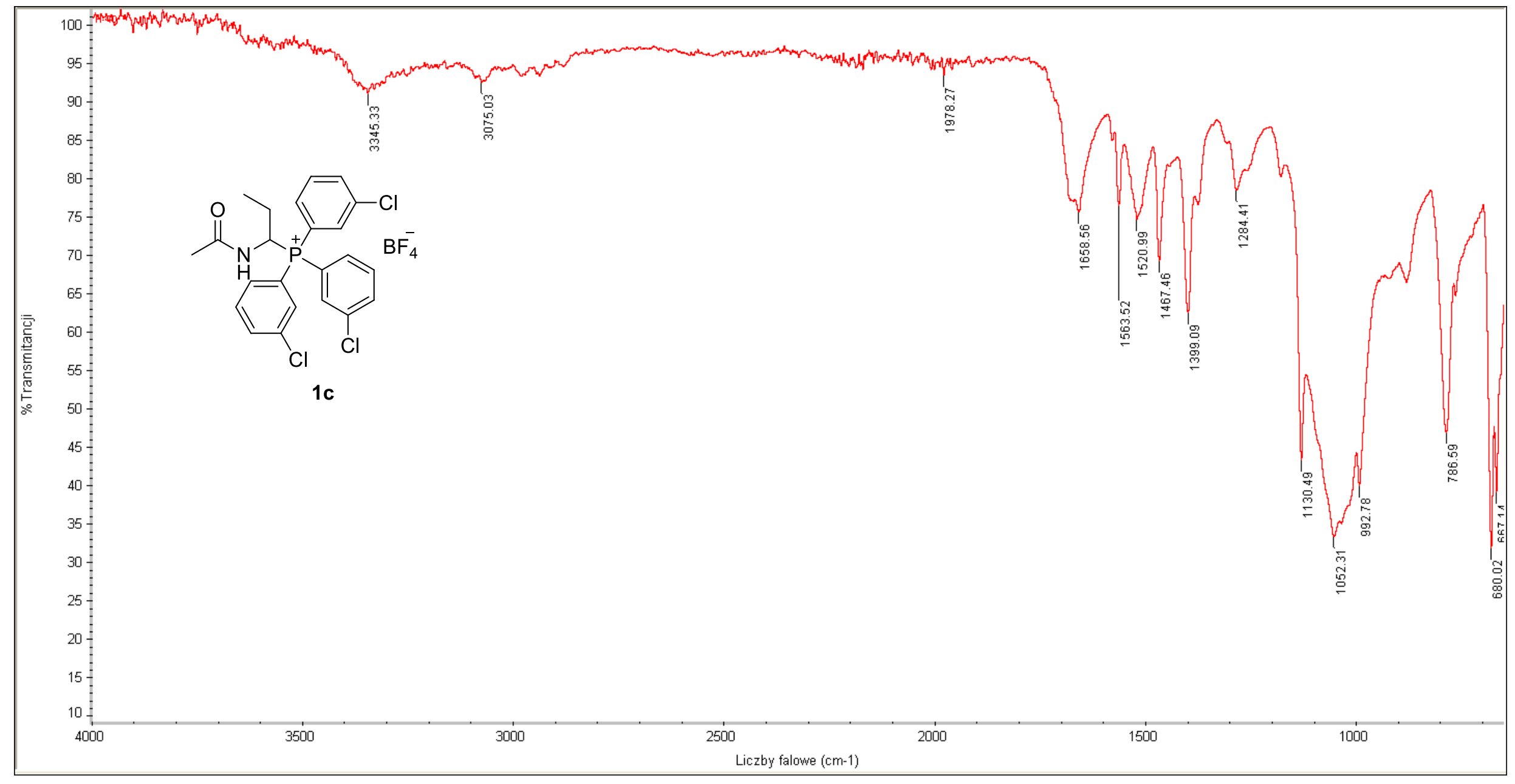

IR spectrum of 1-(N-acetylamino)propyltris(3-chlorophenyl)phosphonium tetrafluoroborate (1c); ATR $\left(\mathrm{cm}^{-1}\right)$. 


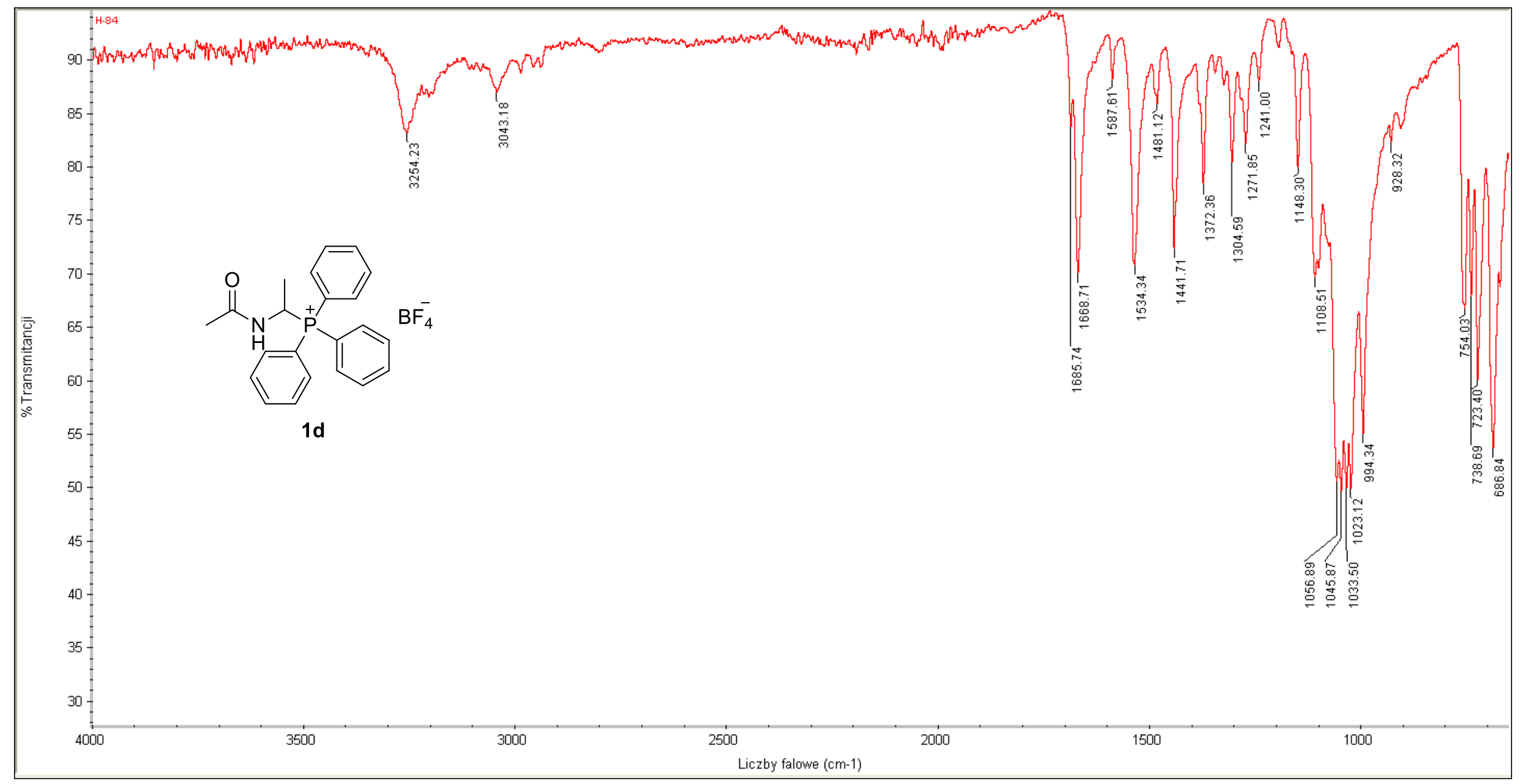

IR spectrum of 1-(N-acetylamino)ethyltriphenylphosphonium tetrafluoroborate $(\mathbf{1 d})$; ATR $\left(\mathrm{cm}^{-1}\right)$. 


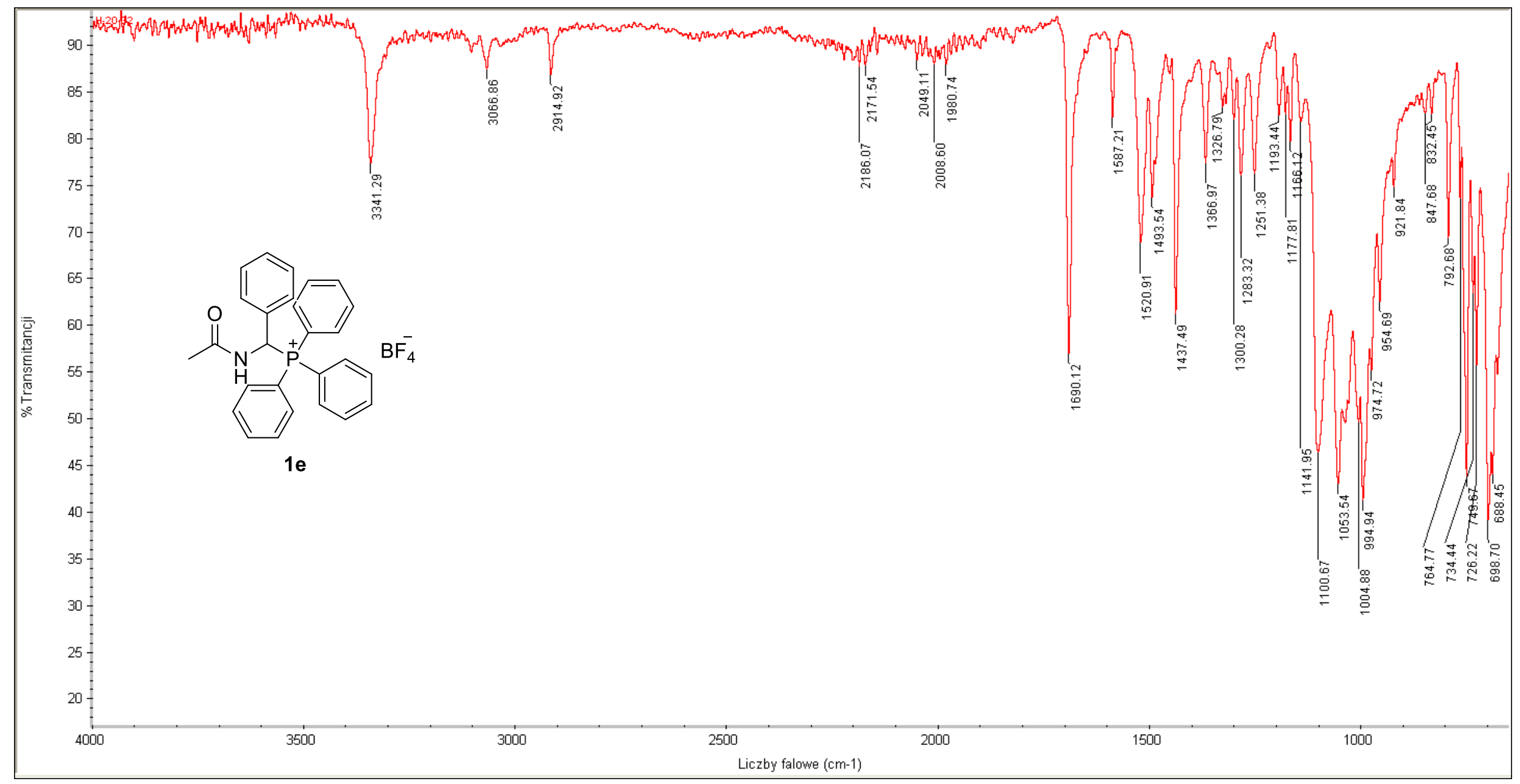

IR spectrum of ( $N$-acetylamino)phenylmethyltriphenylphosphonium tetrafluoroborate $(\mathbf{1 e})$; ATR $\left(\mathrm{cm}^{-1}\right)$. 


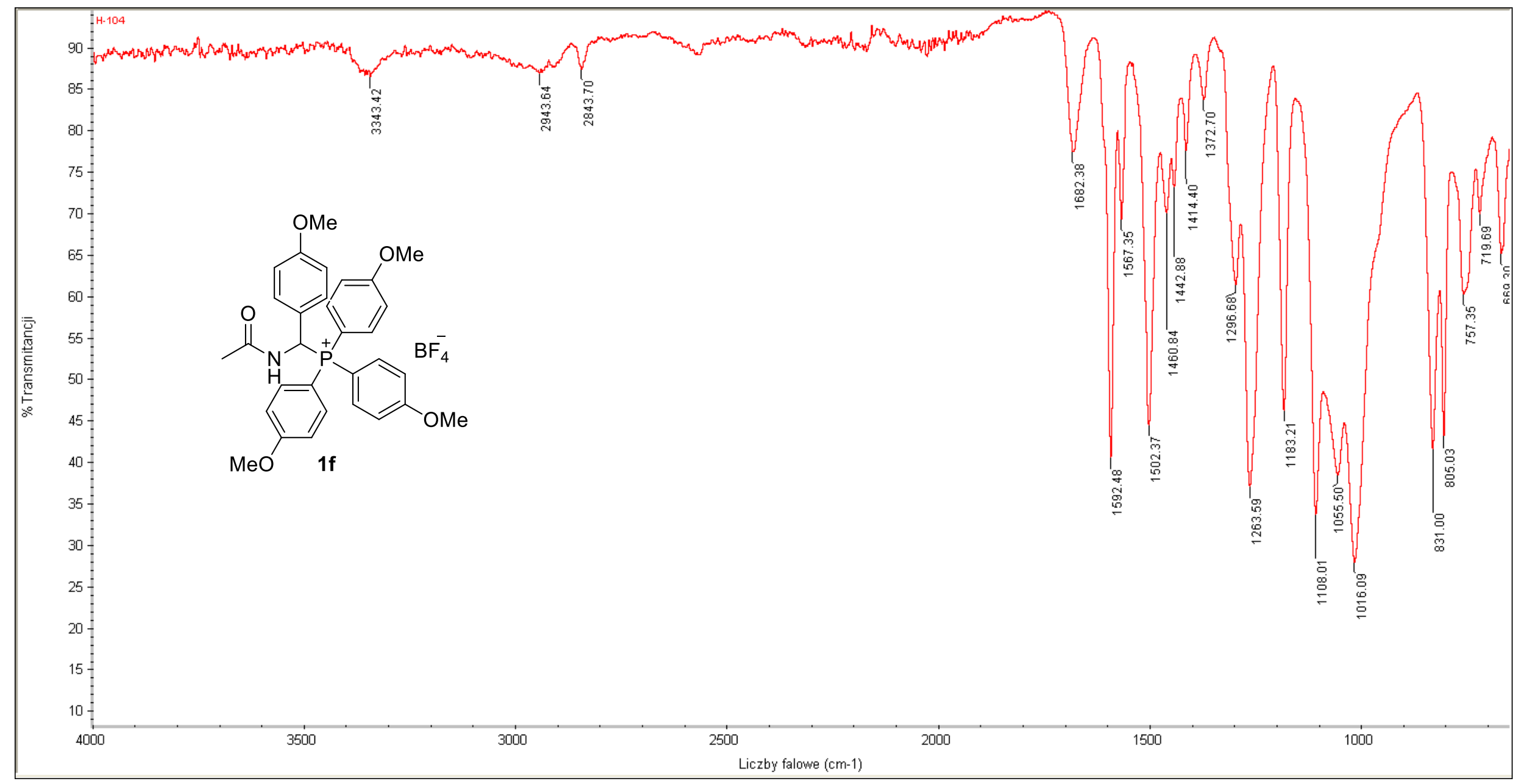

IR spectrum of 1-(N-acetylamino)-1-(4-metoxyphenyl)methyltris(4-metoxyphenyl)phosphonium tetrafluoroborate (1f); ATR (cm $\left.{ }^{-1}\right)$. 


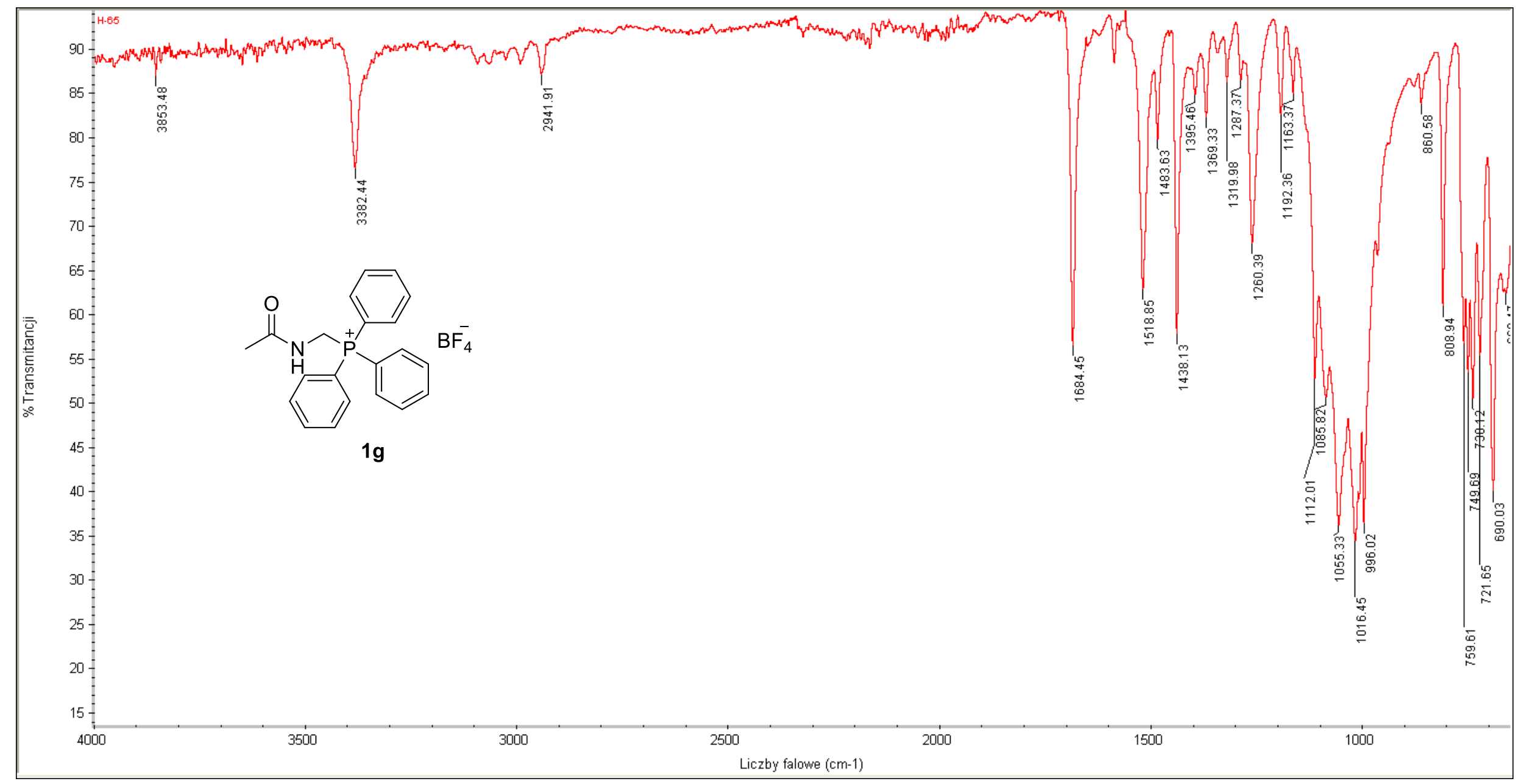

IR spectrum of ( $N$-acetylamino)methyltriphenylphosphonium tetrafluoroborate $(\mathbf{1 g})$; ATR $\left(\mathrm{cm}^{-1}\right)$. 


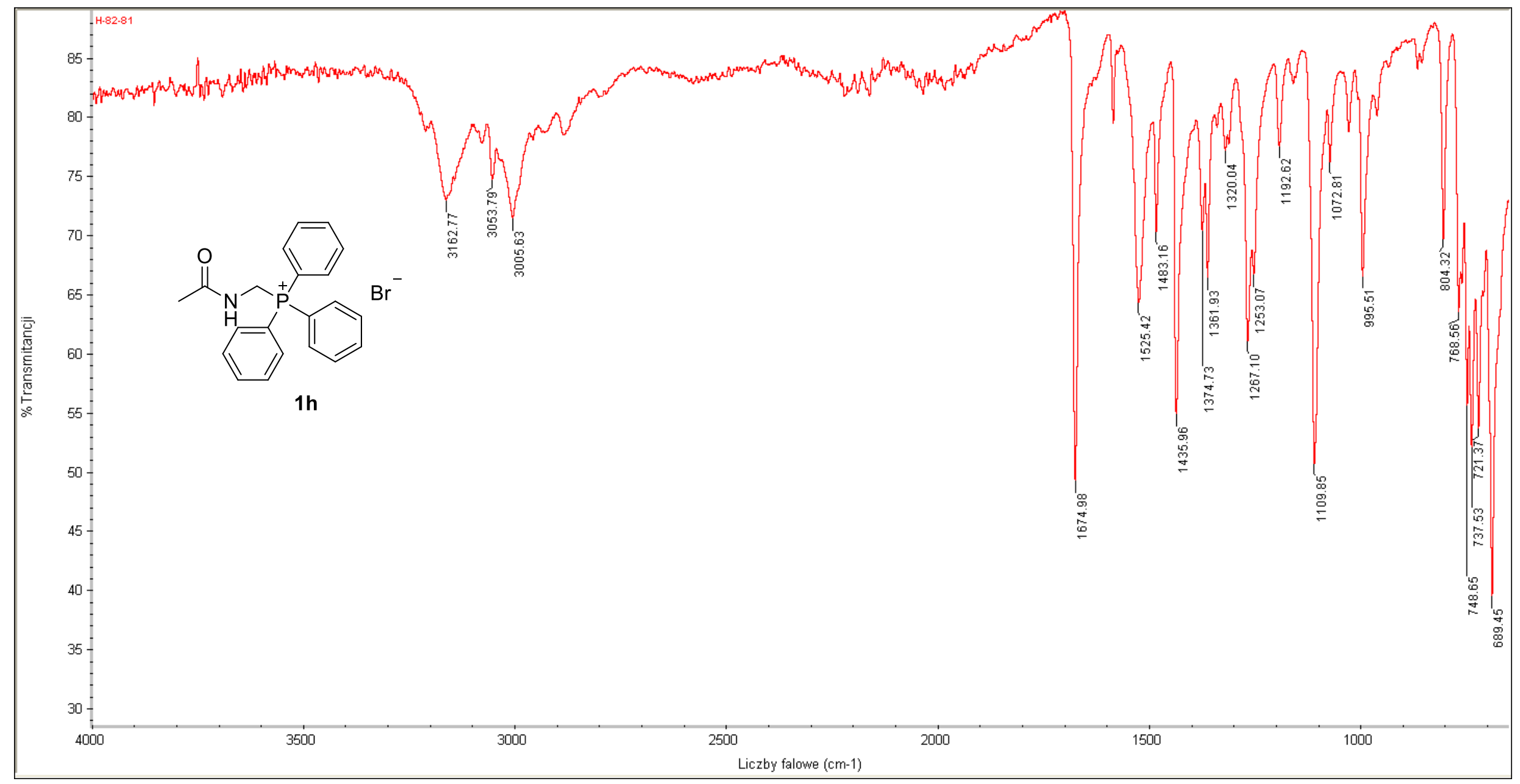

IR spectrum of ( $N$-acetylamino)methyltriphenylphosphonium bromide (1h); ATR $\left(\mathrm{cm}^{-1}\right)$. 


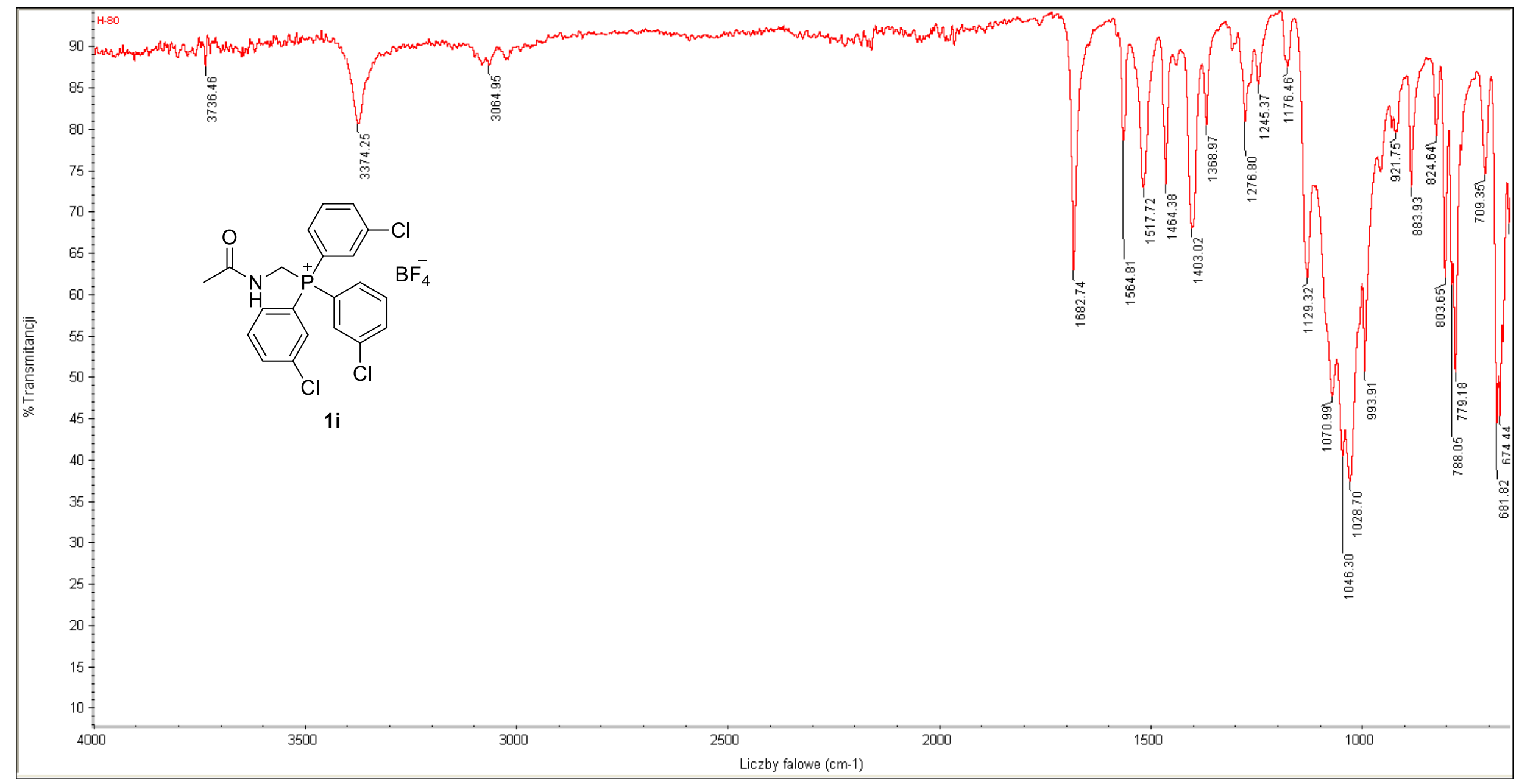

IR spectrum of ( $N$-acetylamino)methyltris(3-chlorophenyl)phosphonium tetrafluoroborate (1i); ATR $\left(\mathrm{cm}^{-1}\right)$. 


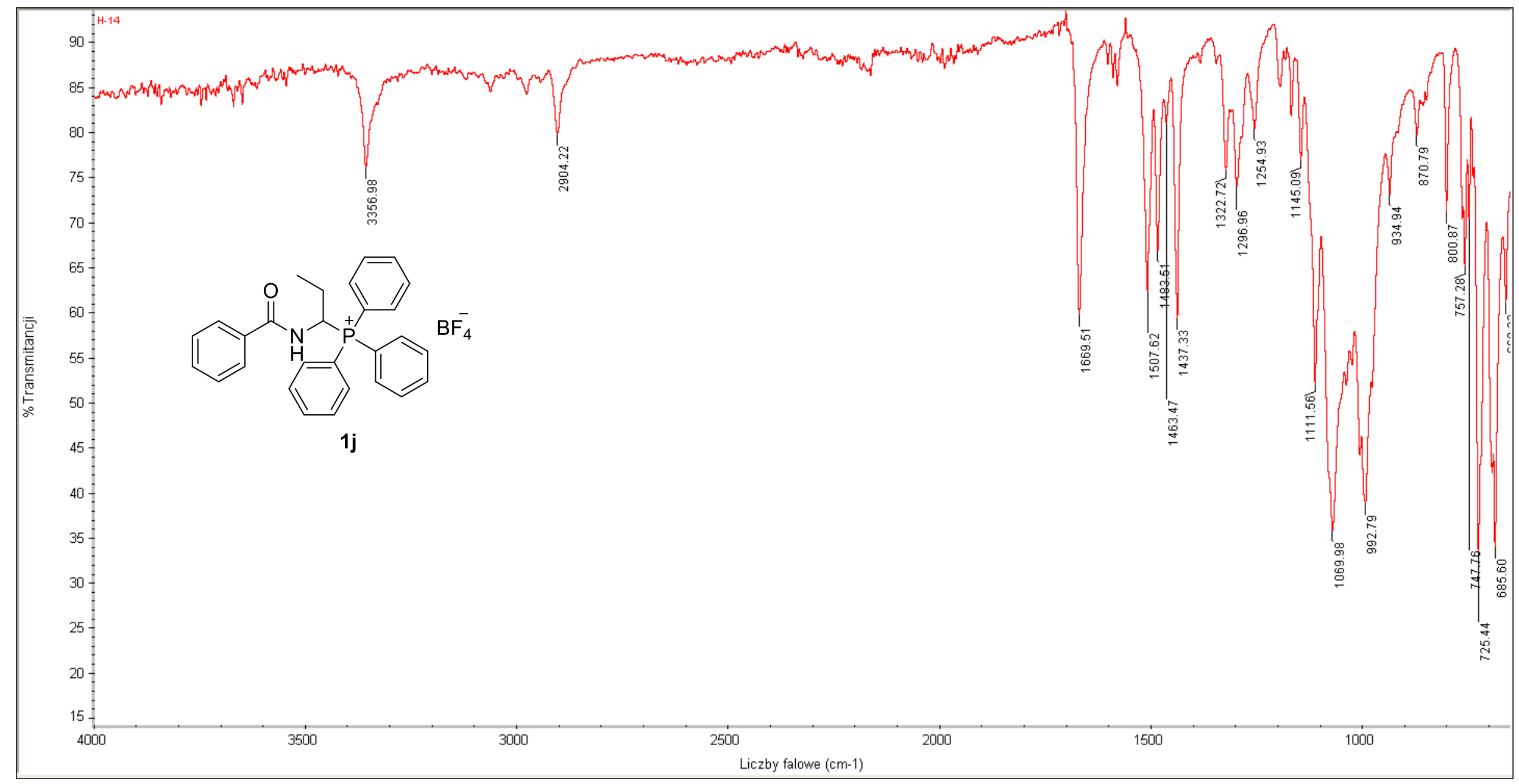

IR spectrum of 1-( $N$-benzoylamino)propyltriphenylphosphonium tetrafluoroborate $(\mathbf{1 j})$; ATR $\left(\mathrm{cm}^{-1}\right)$. 


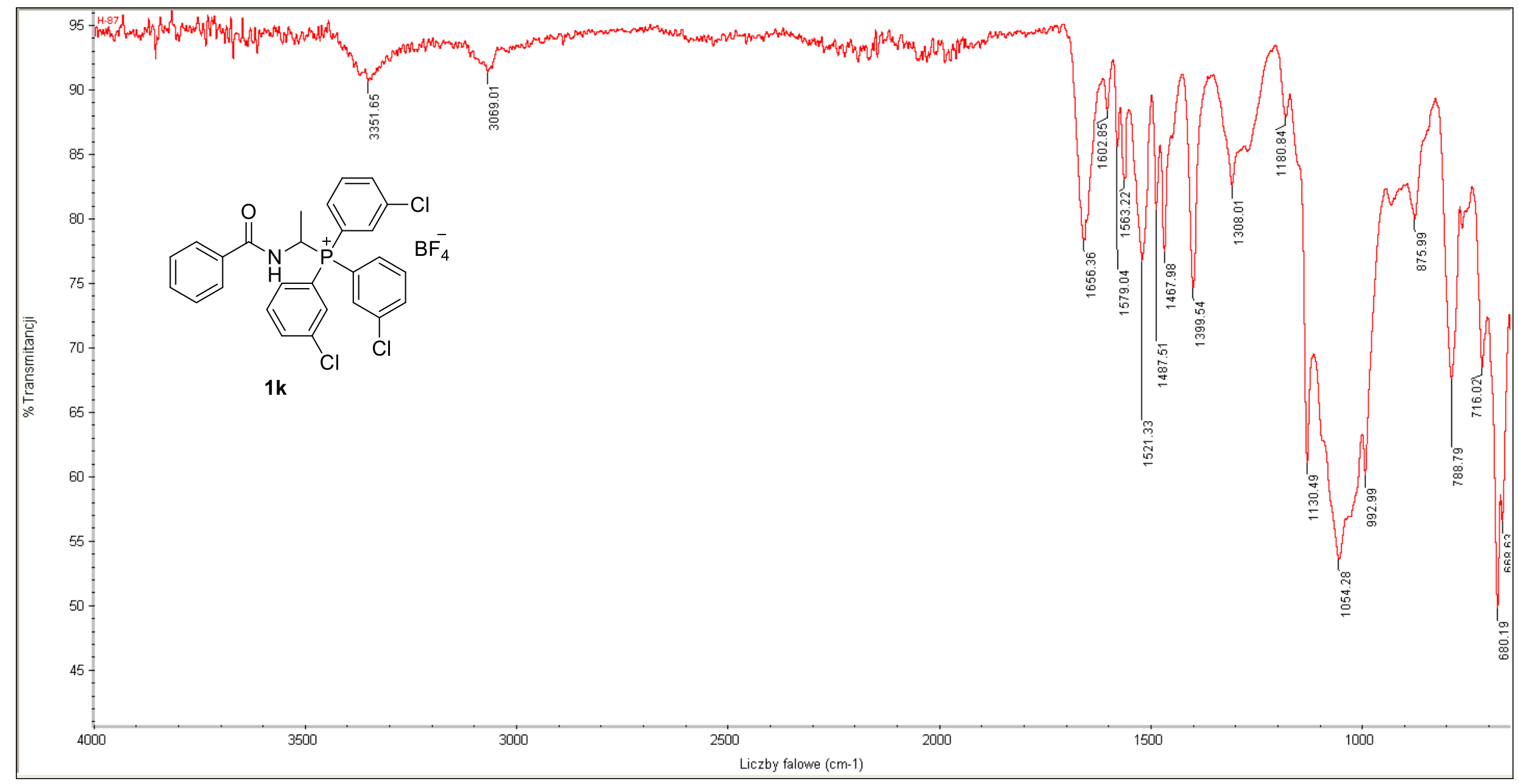

IR spectrum of 1-(N-benzoylamino)ethyltris(3-chlorophenyl)phosphonium tetrafluoroborate (1k); ATR $\left(\mathrm{cm}^{-1}\right)$. 


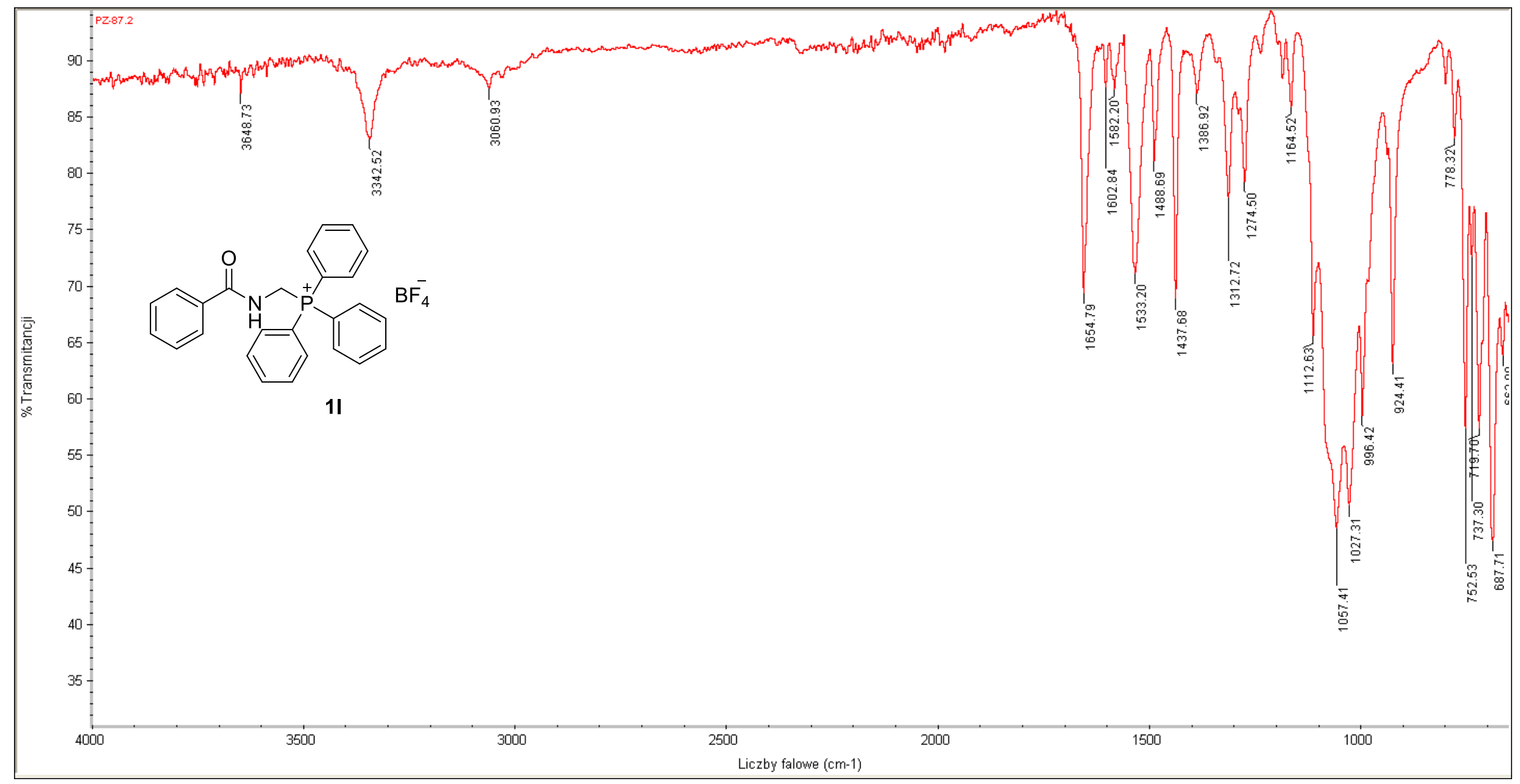

IR spectrum of ( $N$-benzoylamino)methyltriphenylphosphonium tetrafluoroborate (11); ATR $\left(\mathrm{cm}^{-1}\right)$. 


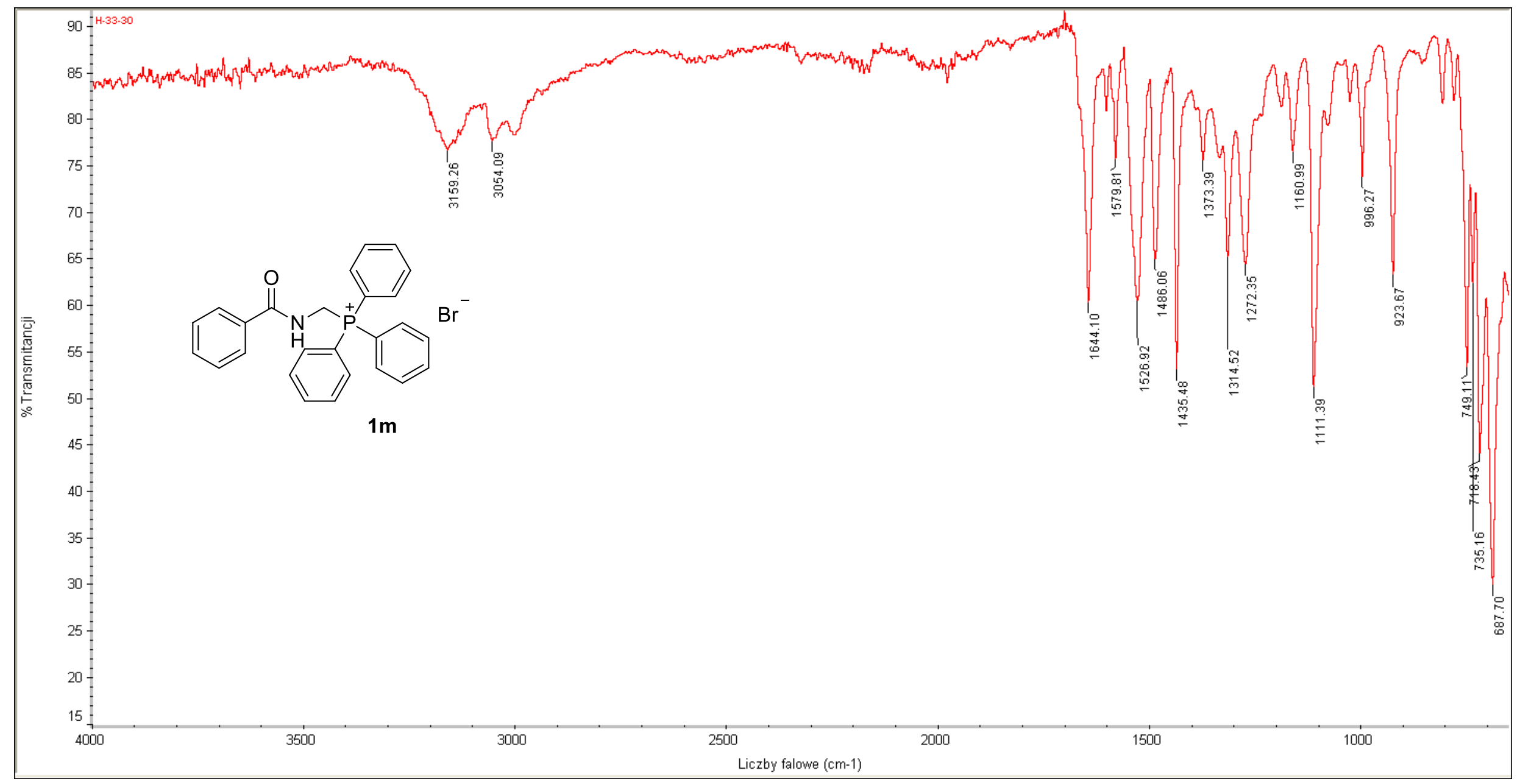

IR spectrum of ( $N$-benzoylamino)methyltriphenylphosphonium bromide $(\mathbf{1 m})$; ATR $\left(\mathrm{cm}^{-1}\right)$. 


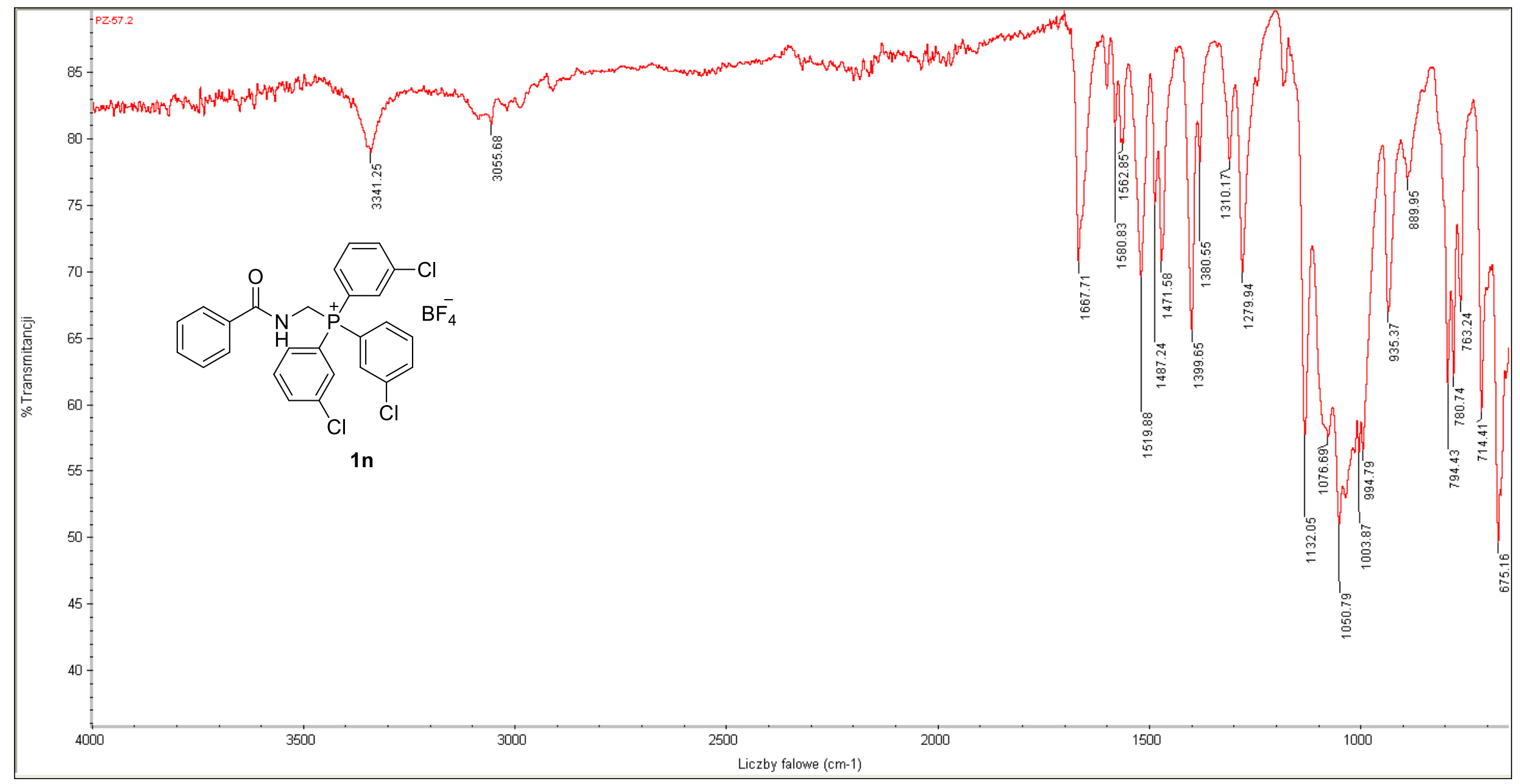

IR spectrum of ( $N$-benzoylamino)methyltris(3-chlorophenyl)phosphonium tetrafluoroborate (1n); ATR $\left(\mathrm{cm}^{-1}\right)$. 


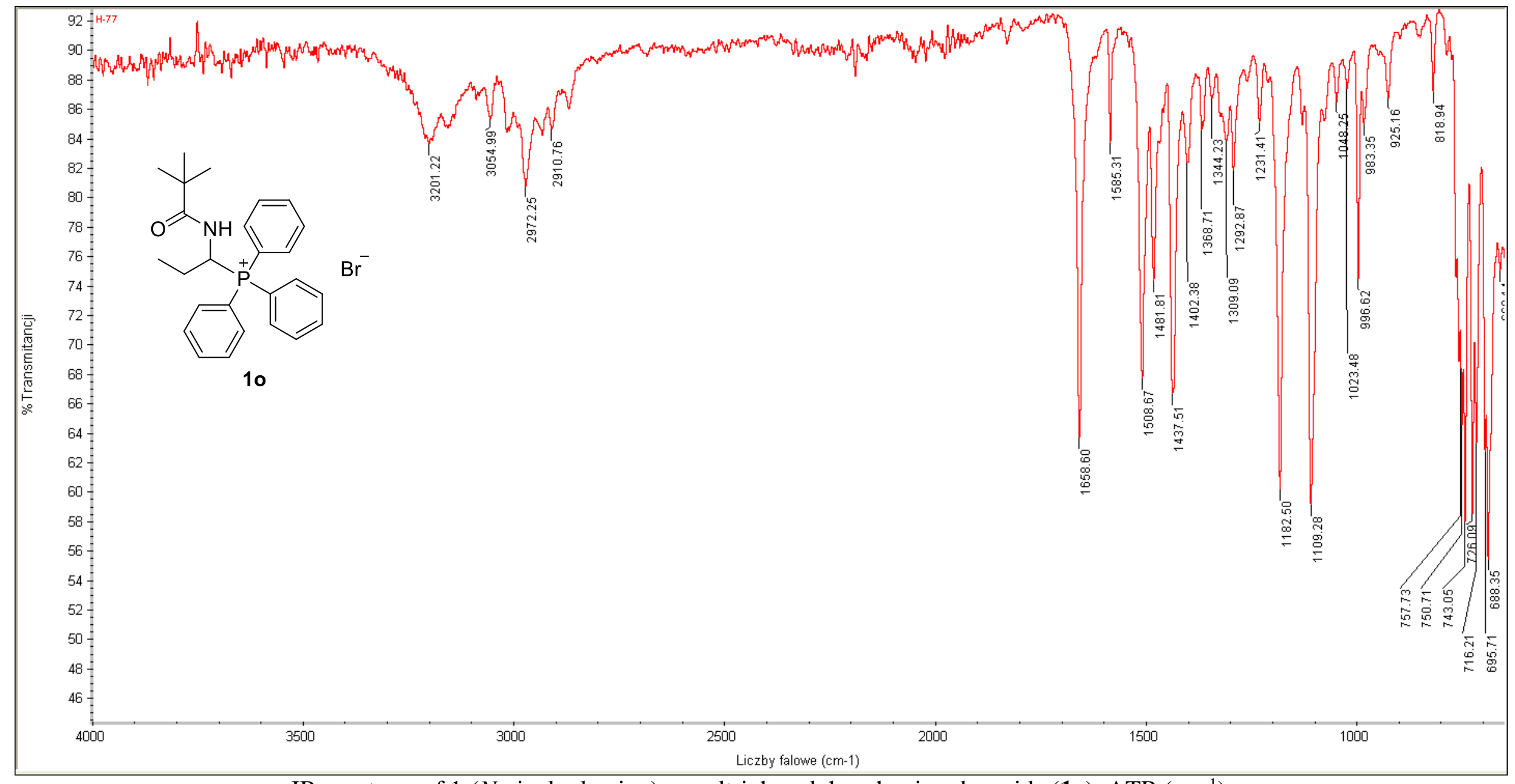

IR spectrum of 1-( $N$-pivaloylamino)propyltriphenylphosphonium bromide (1o); ATR $\left(\mathrm{cm}^{-1}\right)$. 


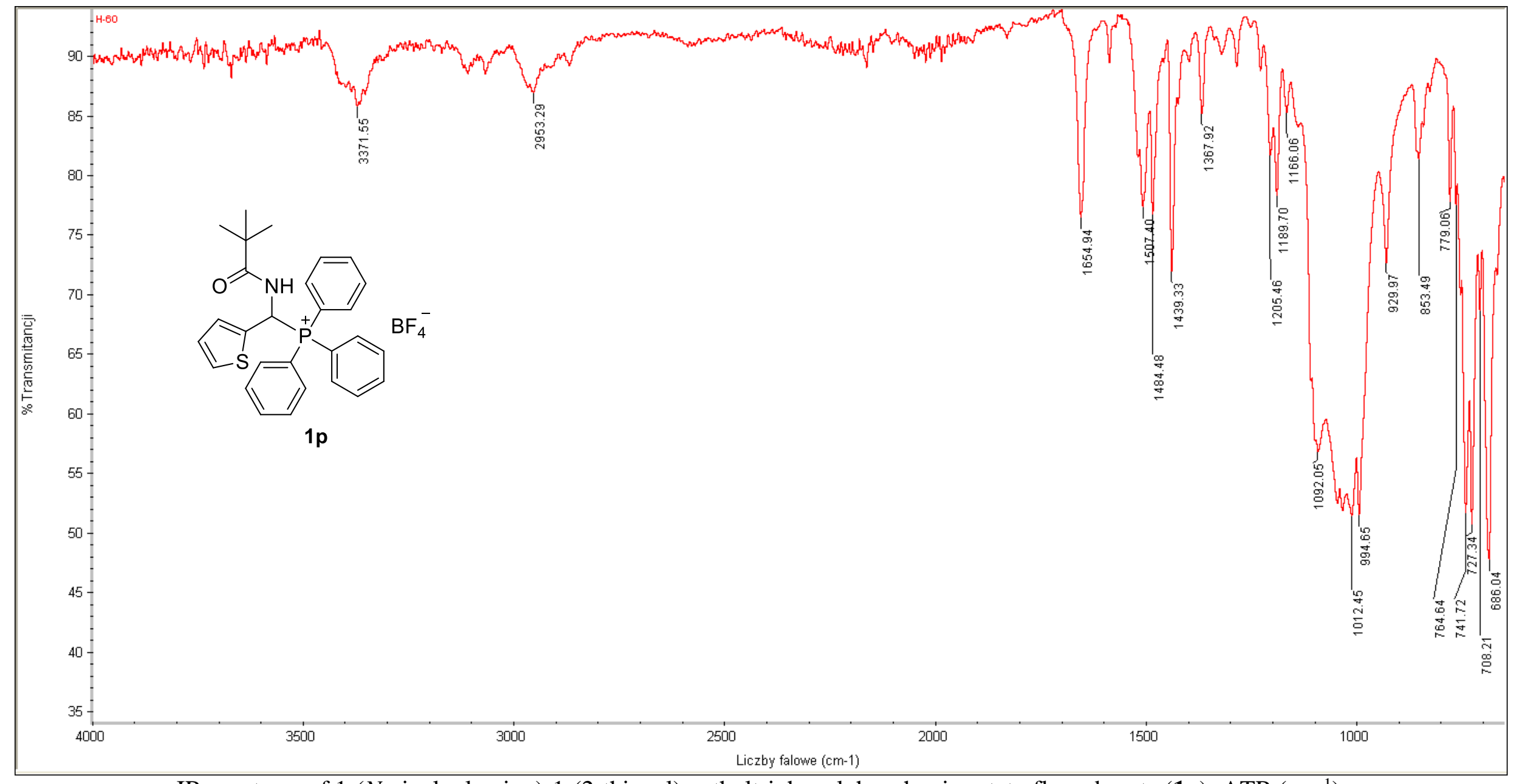

IR spectrum of 1-(N-pivaloylamino)-1-(2-thienyl)methyltriphenylphosphonium tetrafluoroborate (1p); ATR $\left(\mathrm{cm}^{-1}\right)$. 


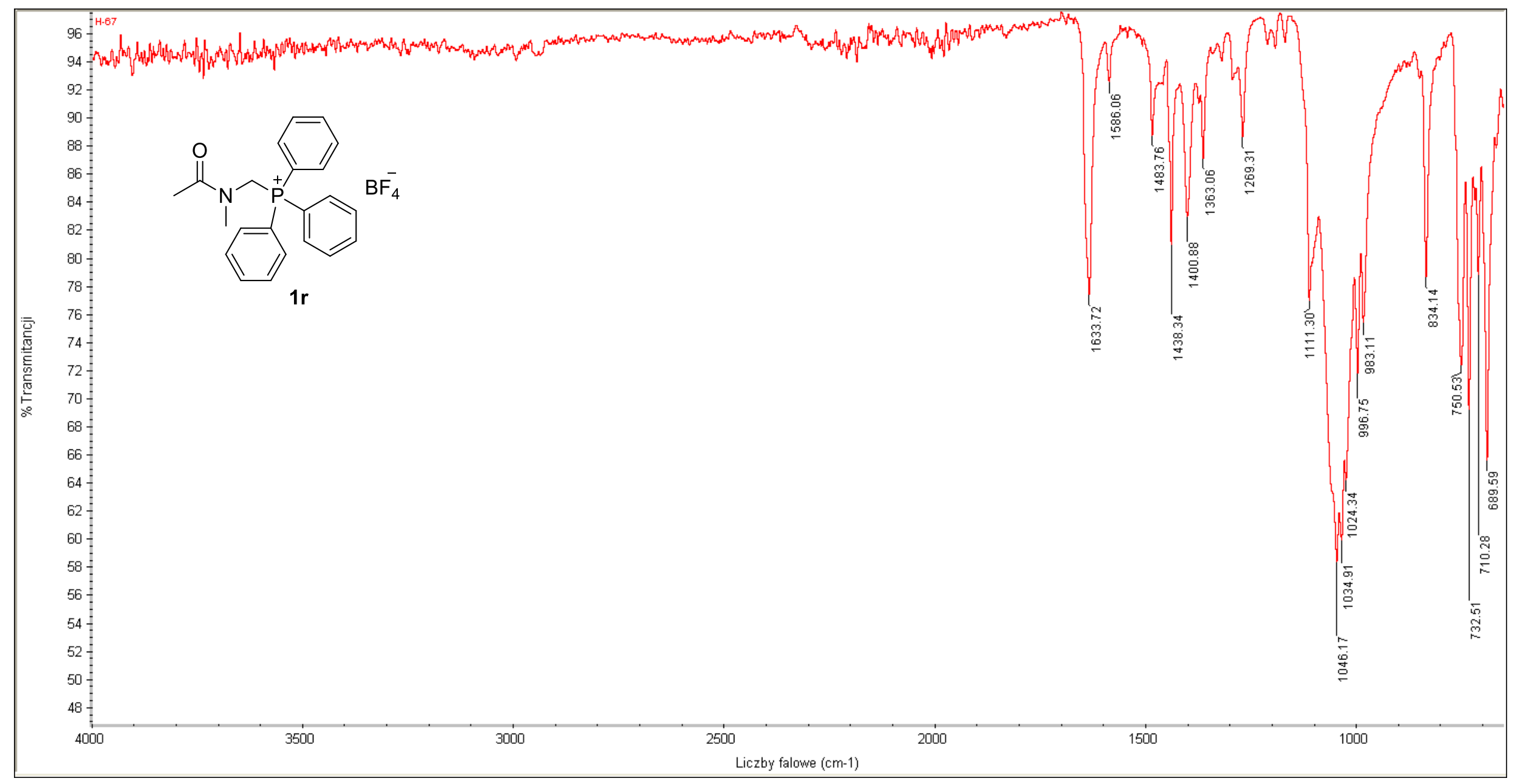

IR spectrum of $N$-( $N$-methylacetylamino)methyltriphenylphosphonium tetrafluoroborate $(\mathbf{1 r})$; ATR $\left(\mathrm{cm}^{-1}\right)$. 


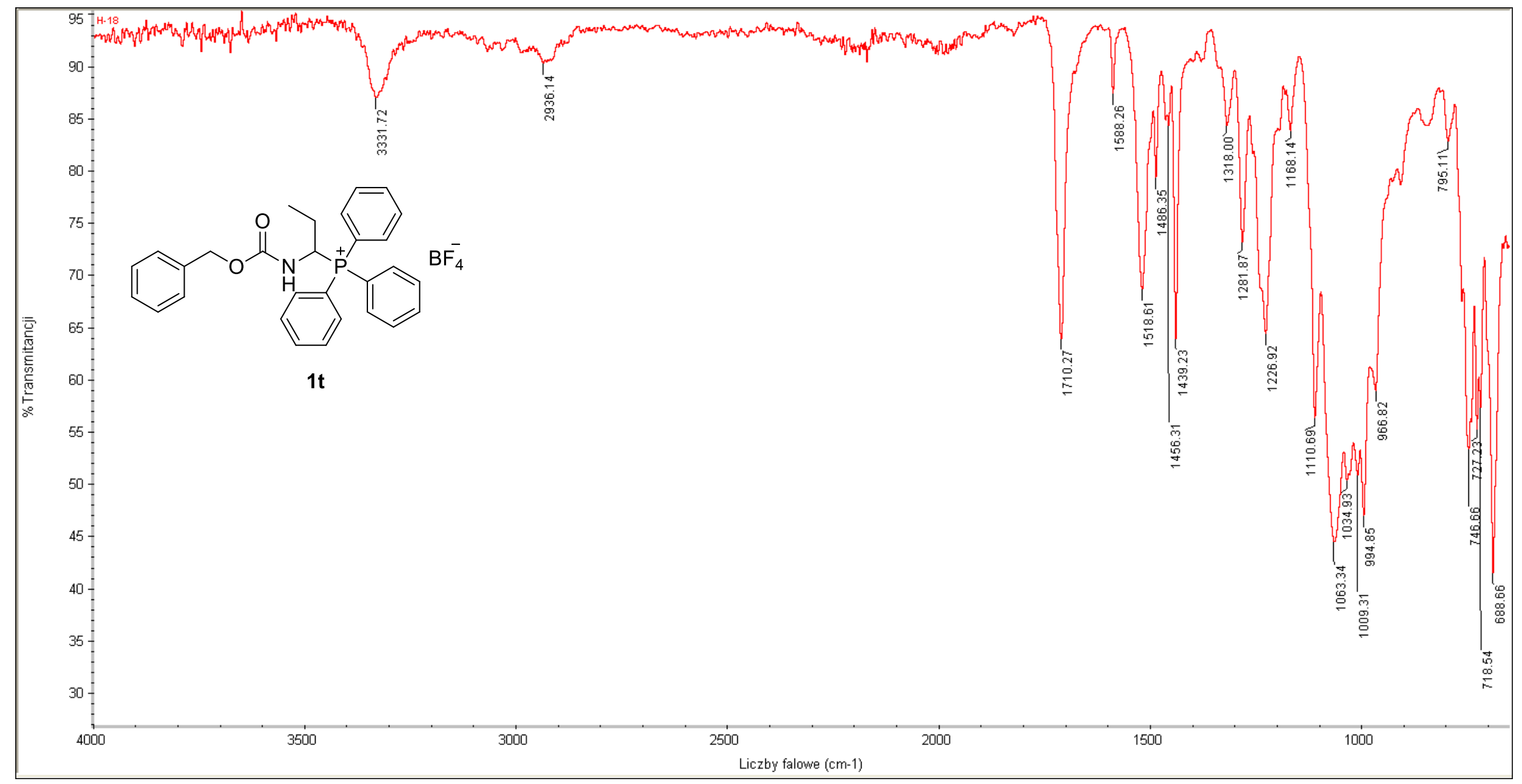

IR spectrum of 1-( $N$-benzyloxycarbonylamino)propyltriphenylphosphonium tetrafluoroborate (1t); ATR $\left(\mathrm{cm}^{-1}\right)$. 


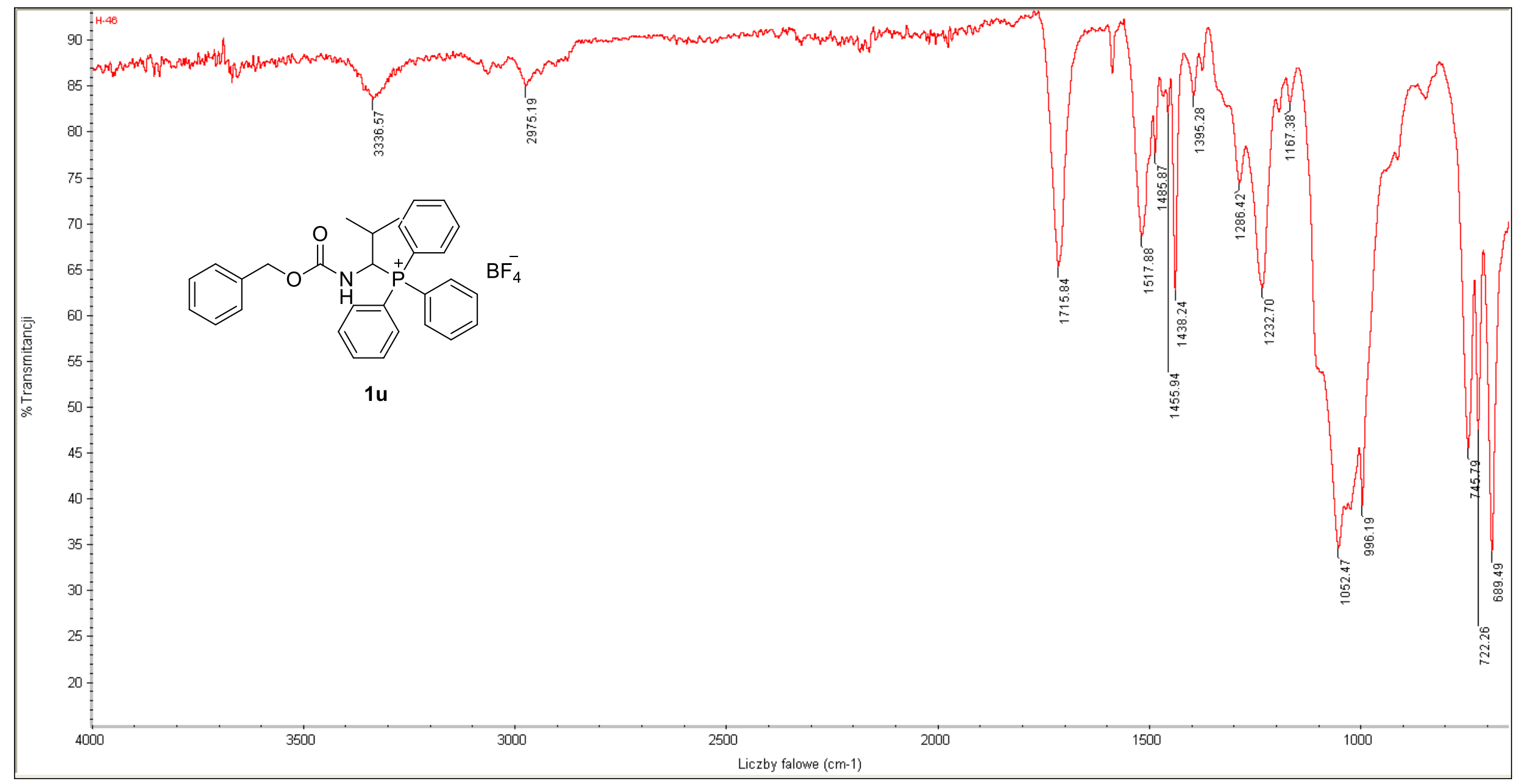

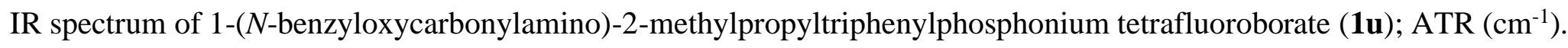




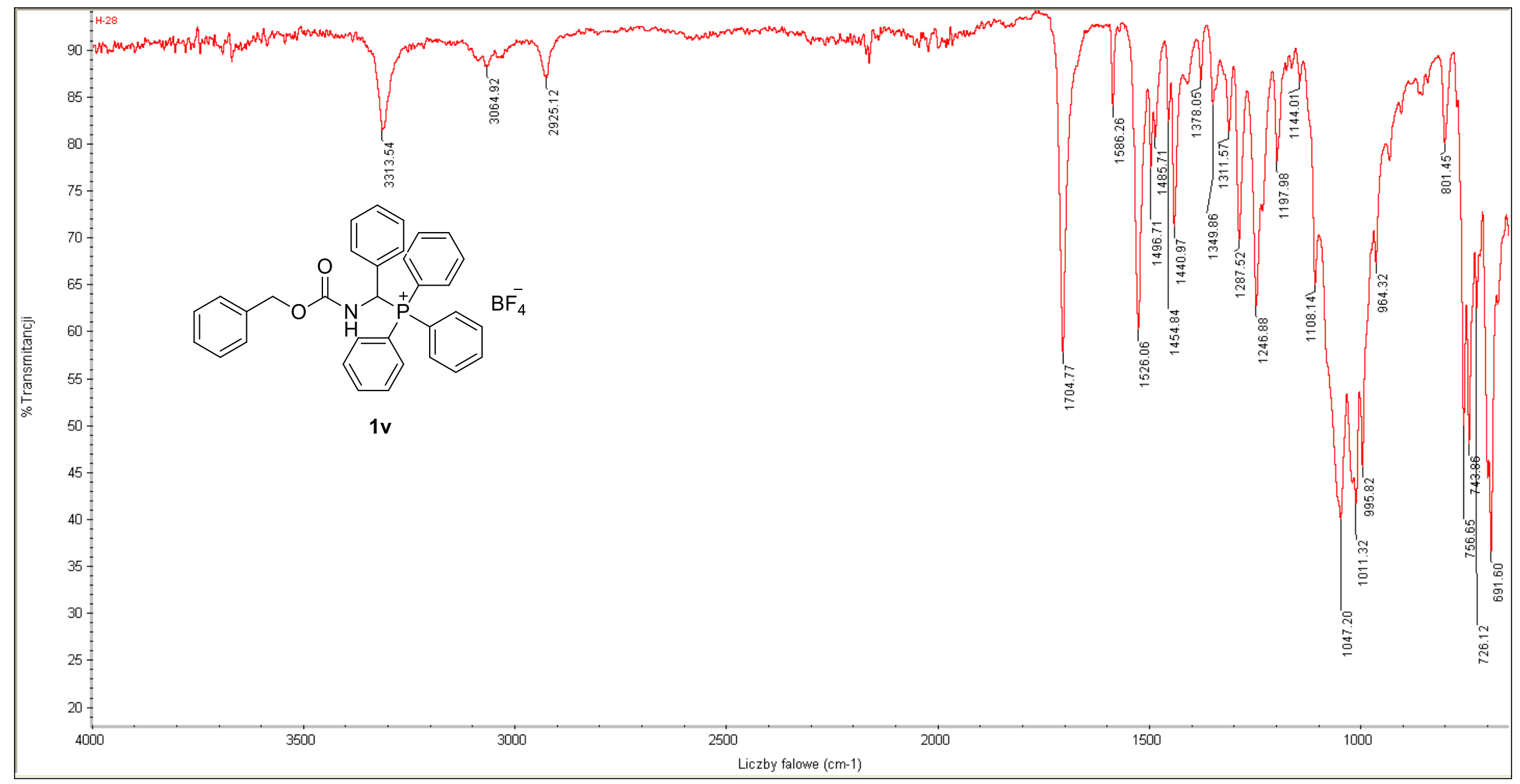

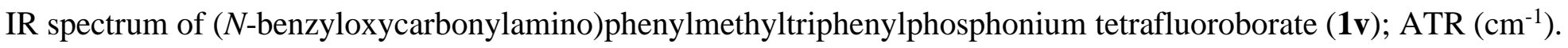




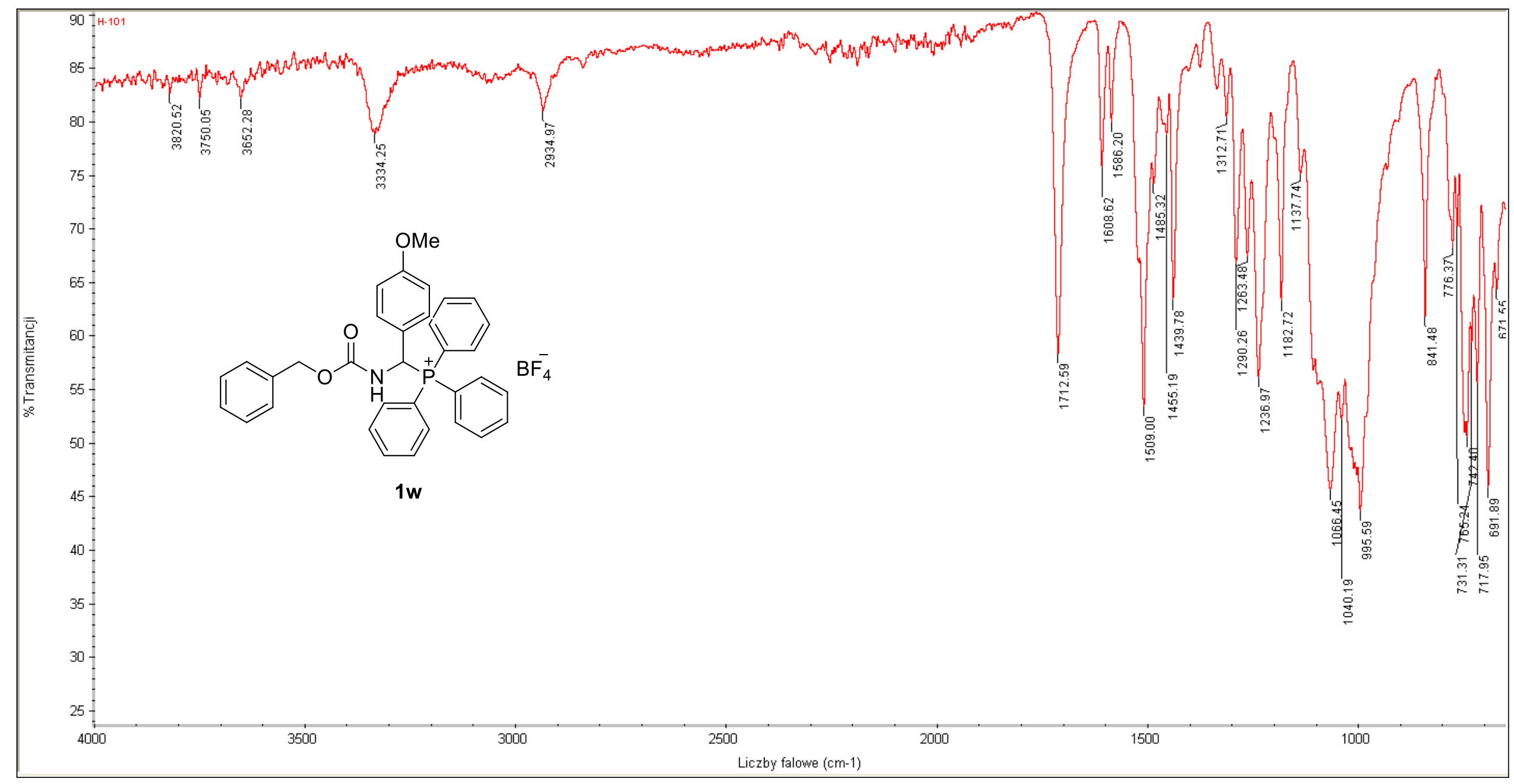

IR spectrum of 1-(N-benzyloxycarbonylamino)-1-(4-methoxyphenyl)methyltriphenylphosphonium tetrafluoroborate (1w); ATR (cm $\left.{ }^{-1}\right)$. 


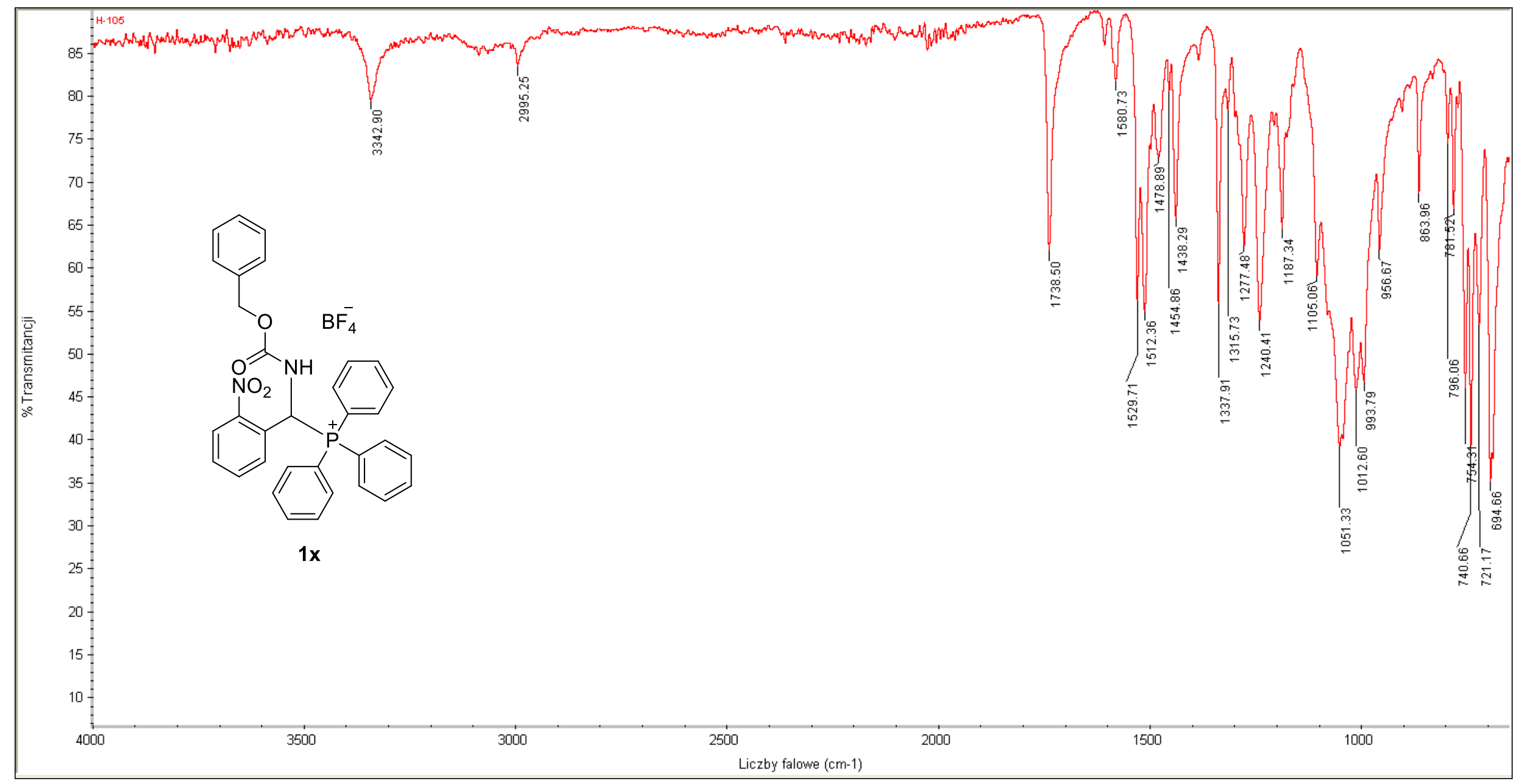

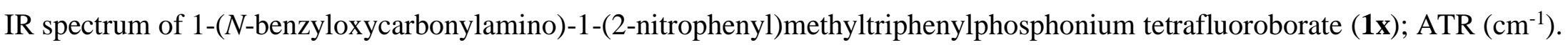




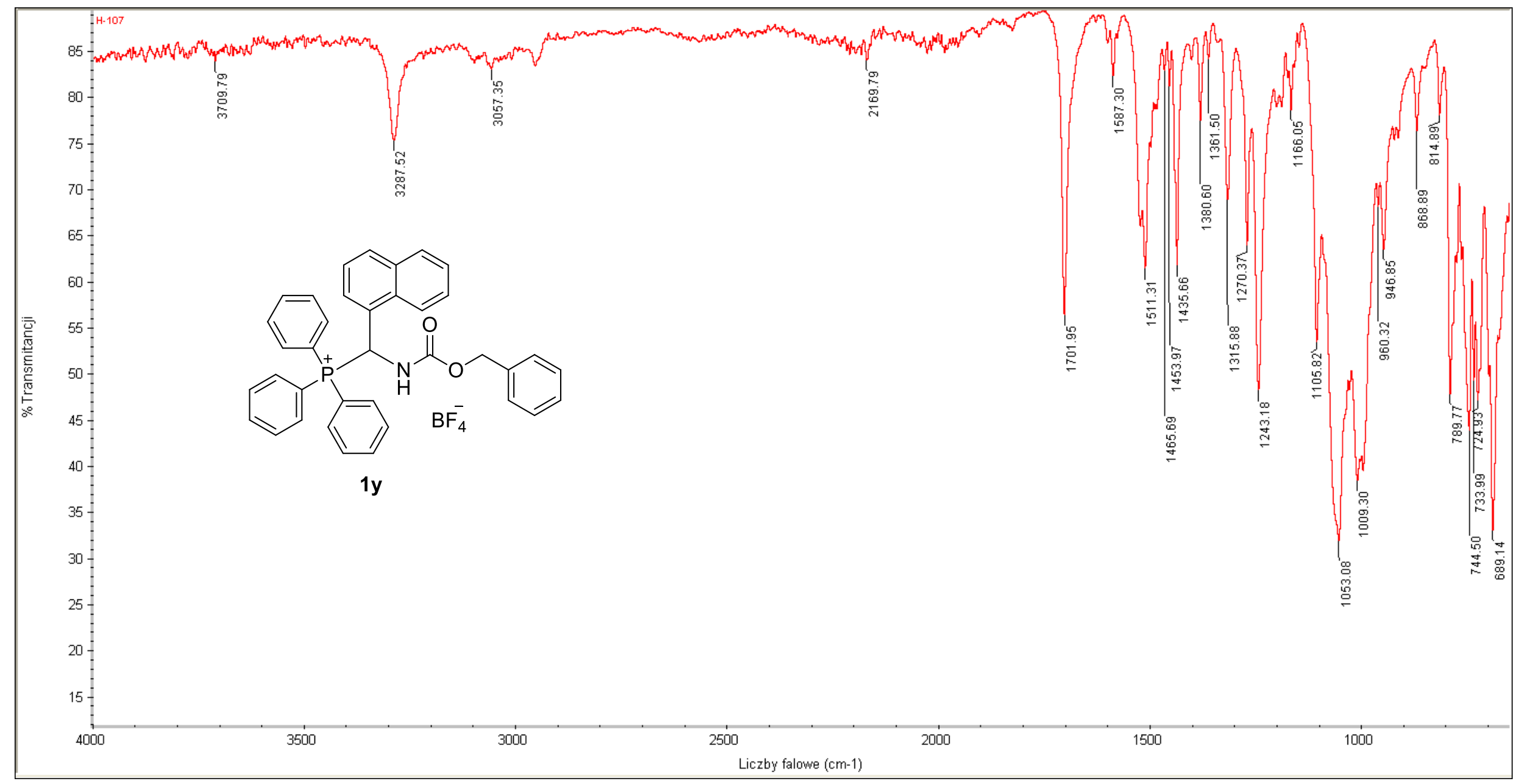

IR spectrum of 1-(N-benzyloxycarbonylamino)-1-(1-naphthyl)methyltriphenylphosphonium tetrafluoroborate (1y); ATR (cm $\left.{ }^{-1}\right)$. 


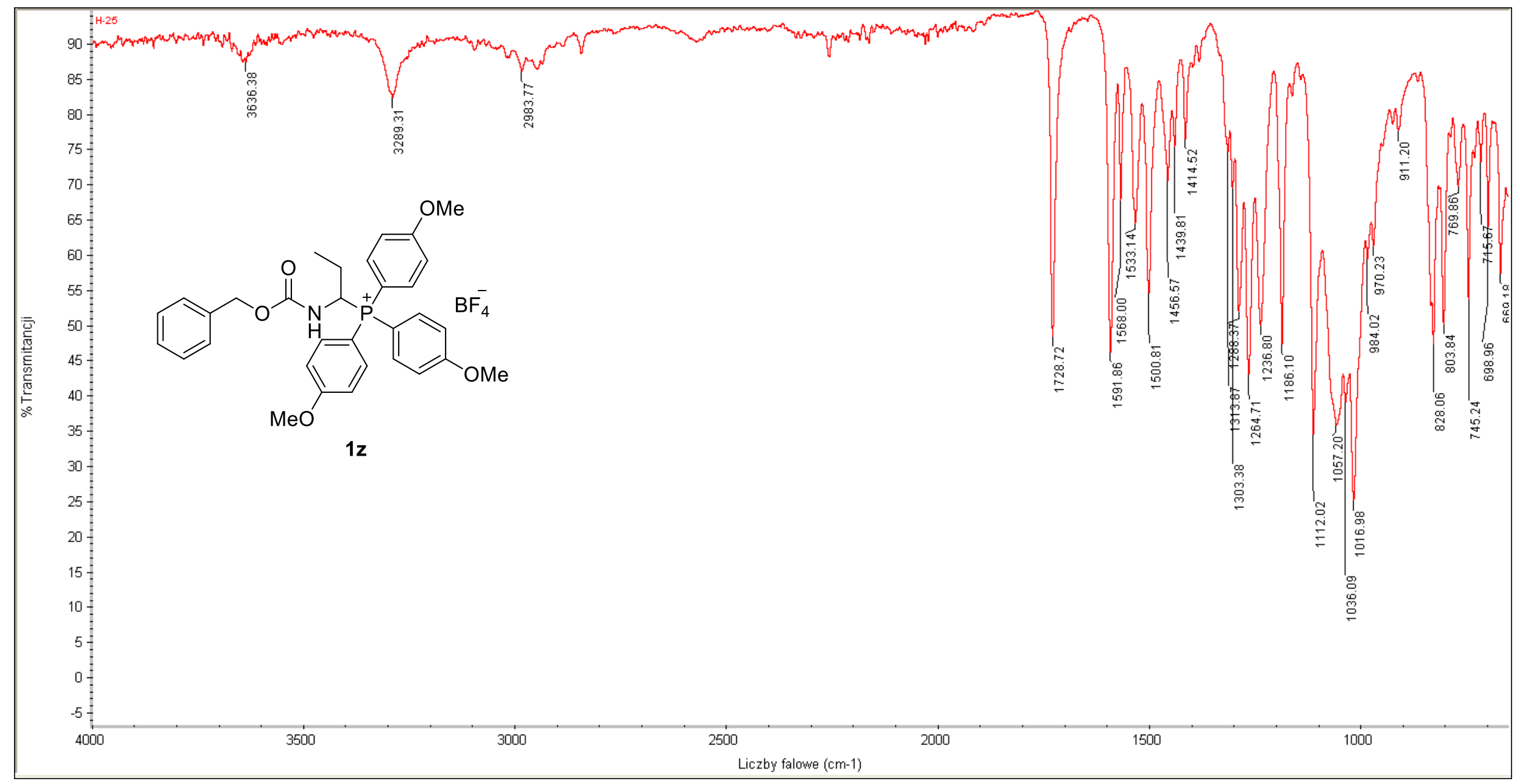

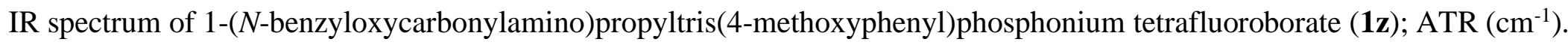




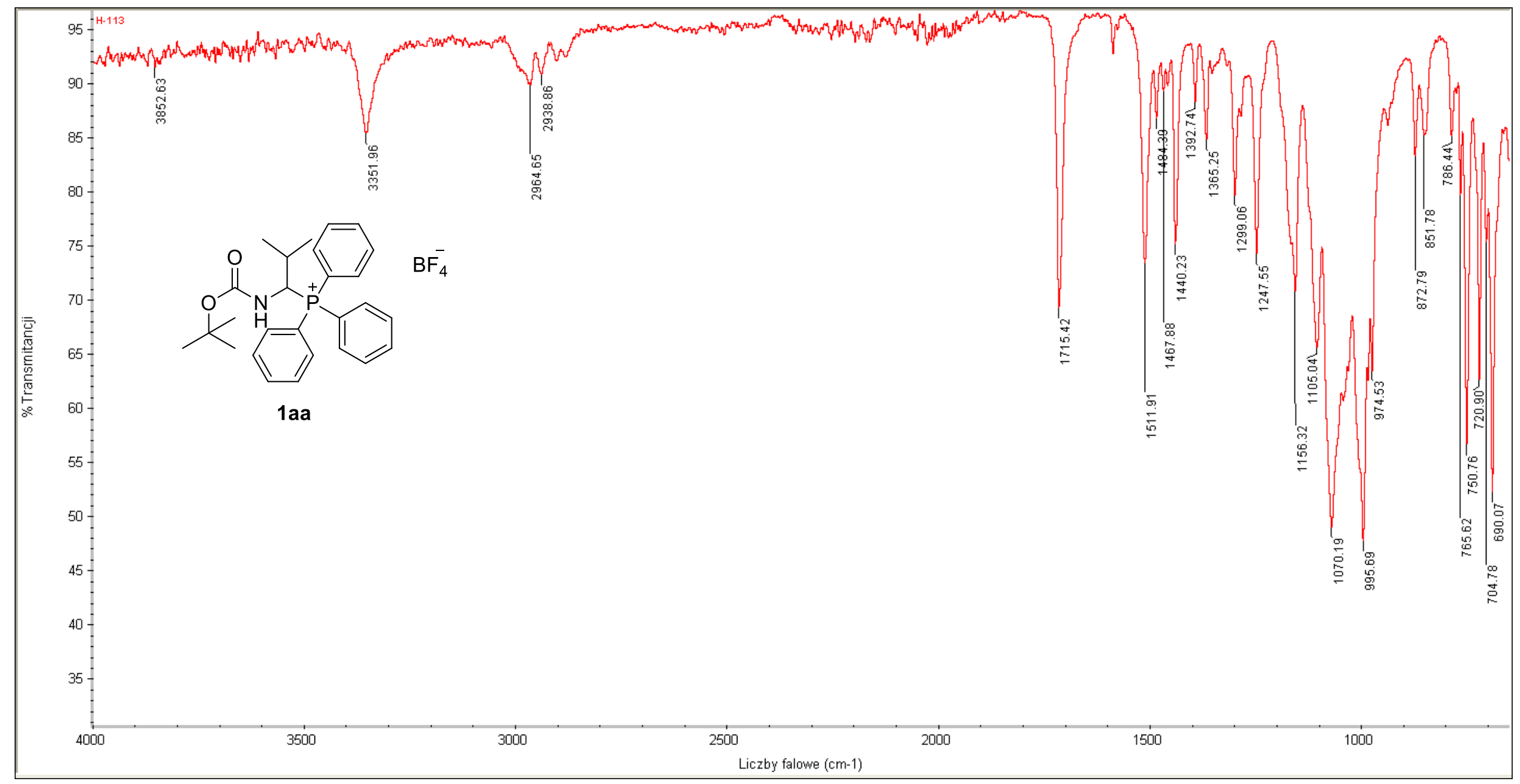

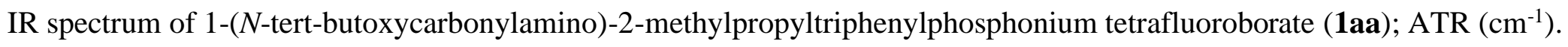




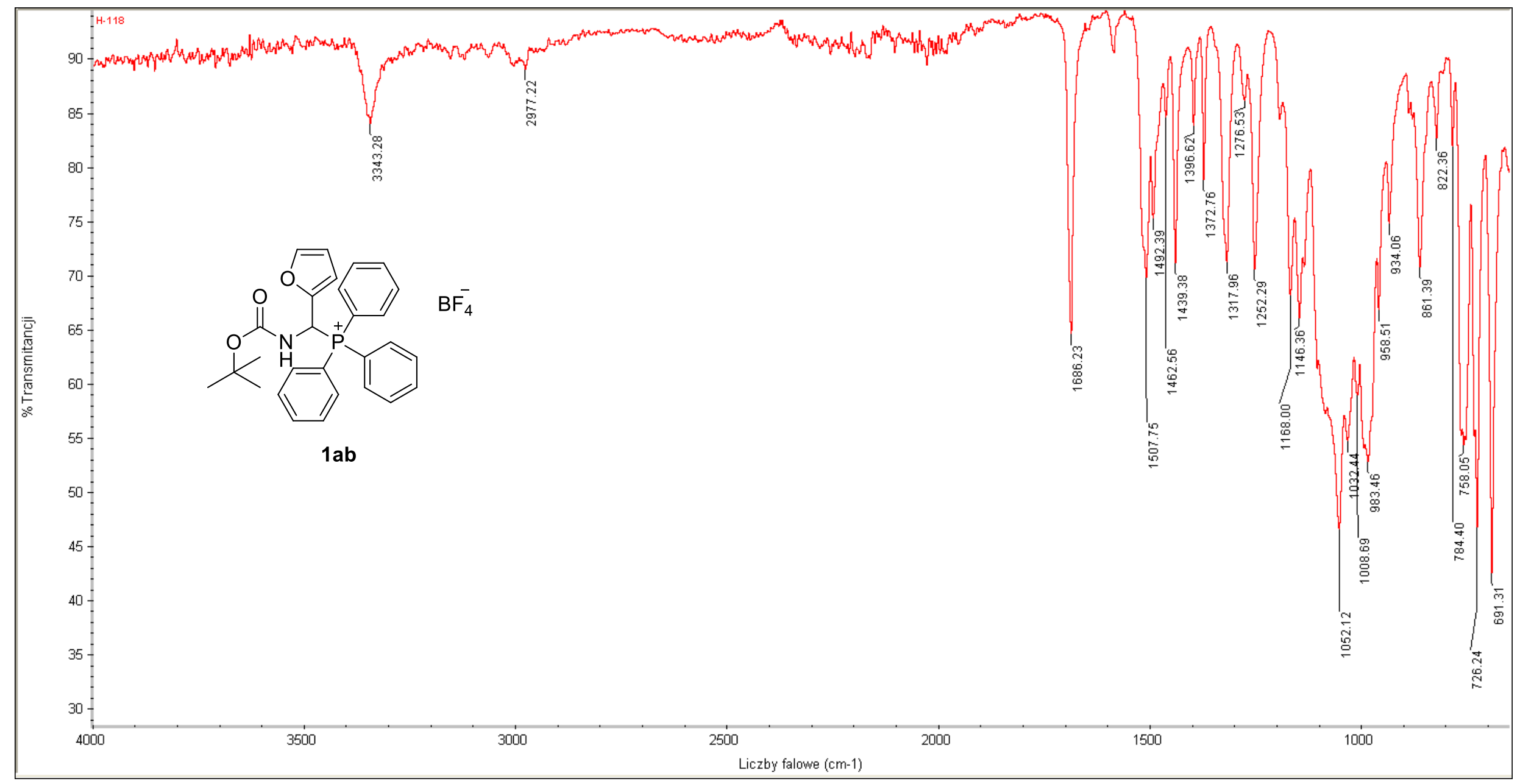

IR spectrum of 1-( $N$-tert-butoxycarbonylamino)-1-(2-furyl)methyltriphenylphosphonium tetrafluoroborate (1ab); ATR $\left(\mathrm{cm}^{-1}\right)$. 


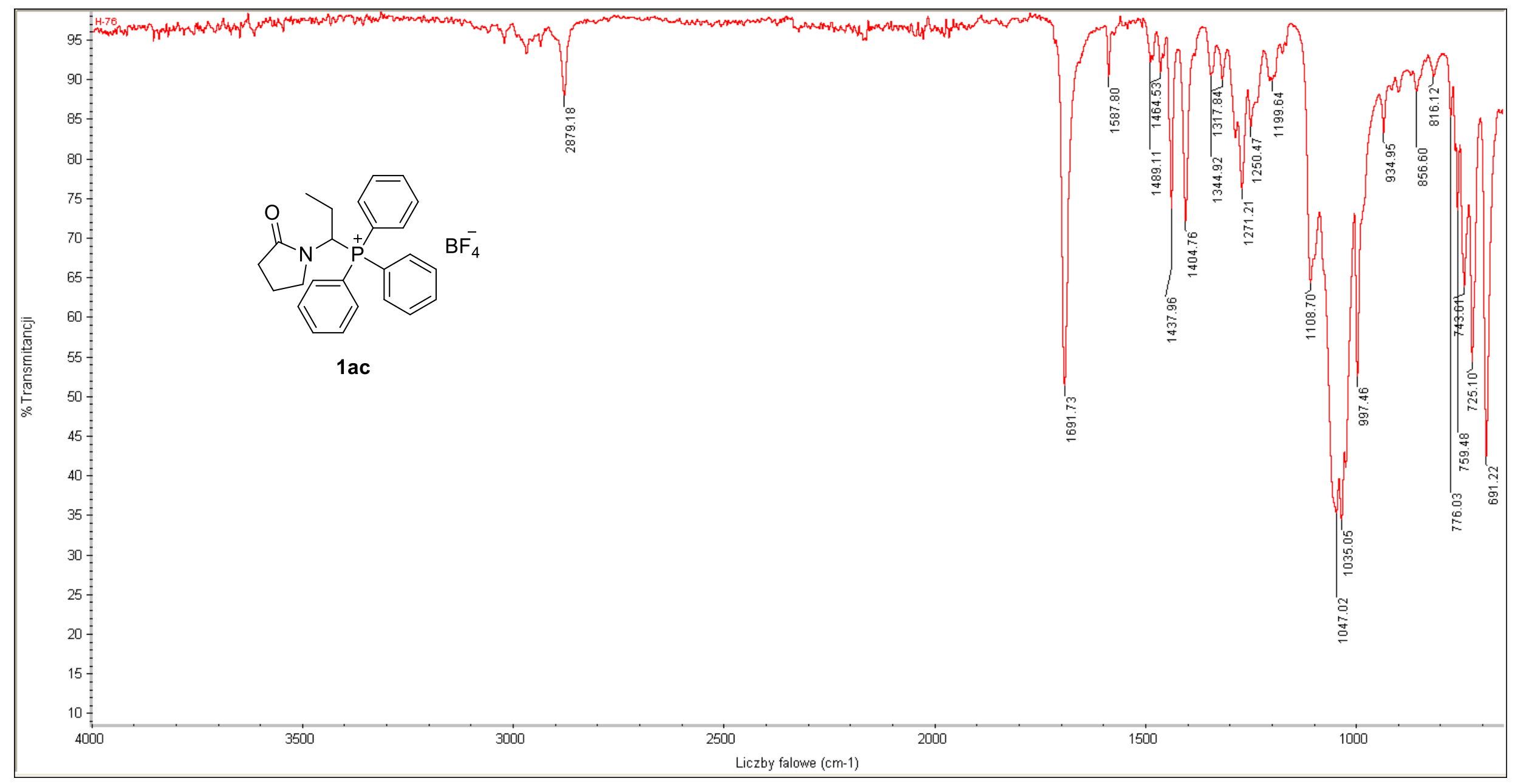

IR spectrum of 1-(2-oxopyrrolidin-1-yl)propyltriphenylphosphonium tetrafluoroborate (1ac); ATR $\left(\mathrm{cm}^{-1}\right)$. 


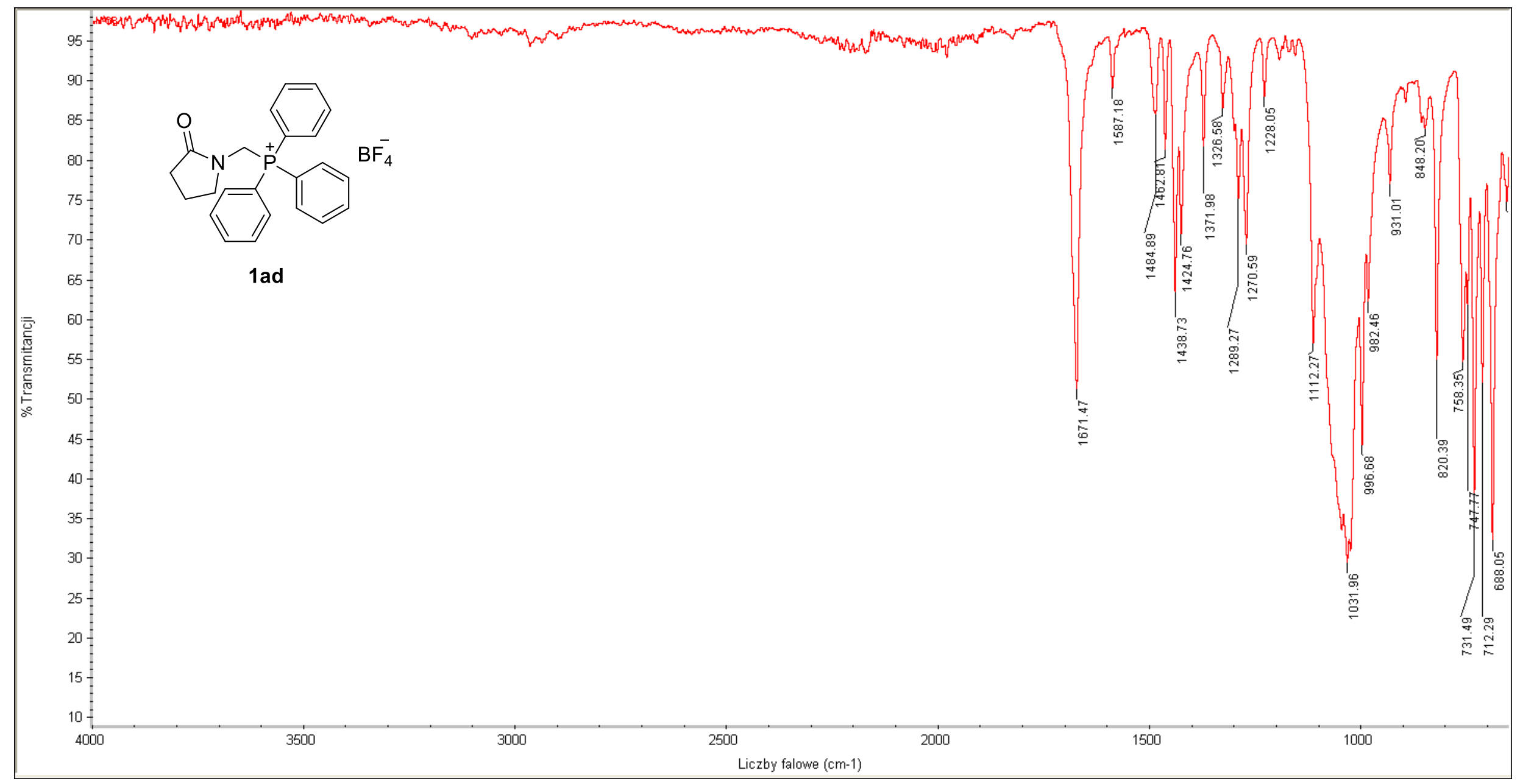

IR spectrum of 1-(2-oxopyrrolidin-1-yl)methyltriphenylphosphonium tetrafluoroborate (1ad); ATR $\left(\mathrm{cm}^{-1}\right)$. 


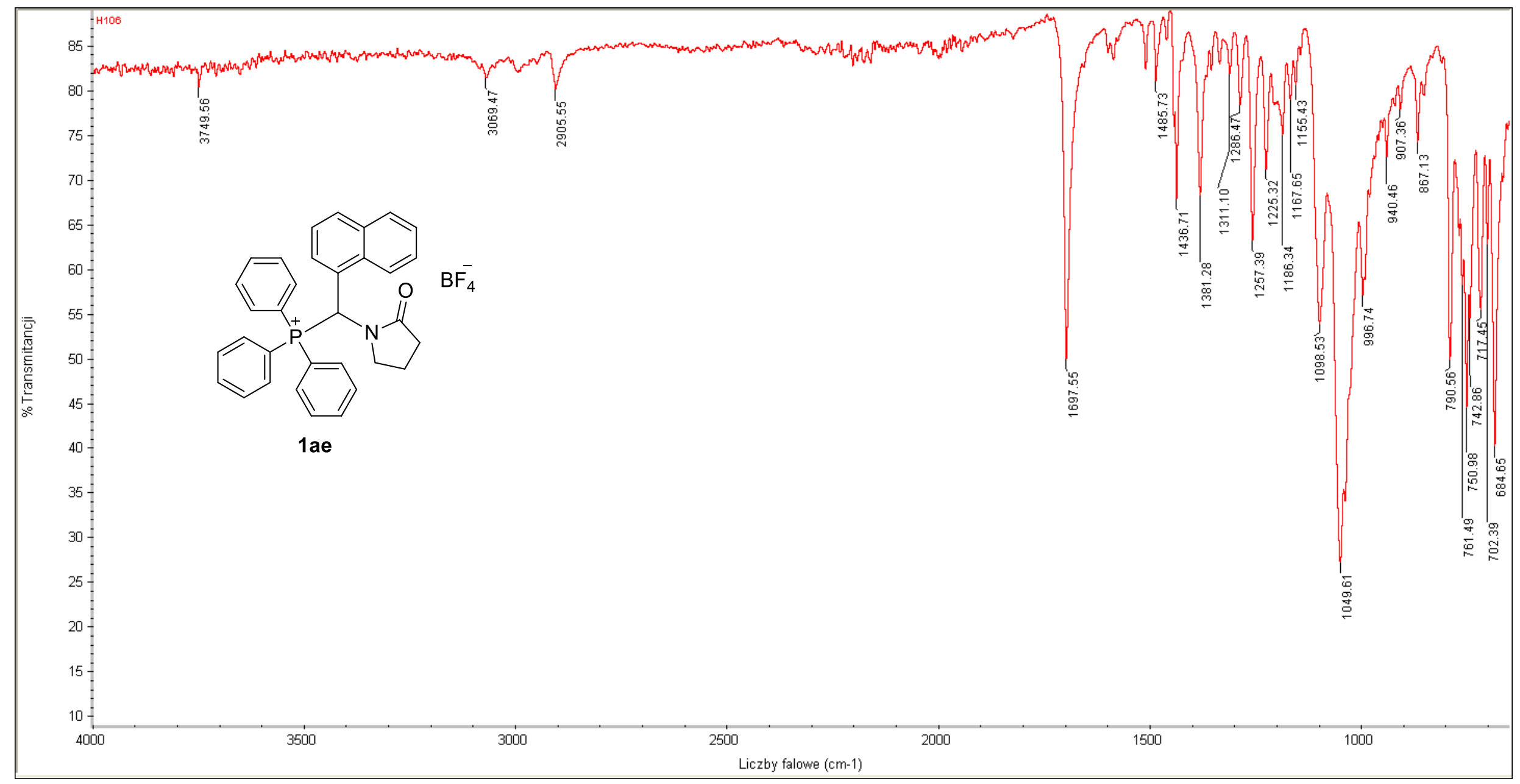

IR spectrum of 1-(2-oxopyrrolidin-1-yl)-1-(1-naphthyl)methyltriphenylphosphonium tetrafluoroborate (1ae); ATR (cm-1). 


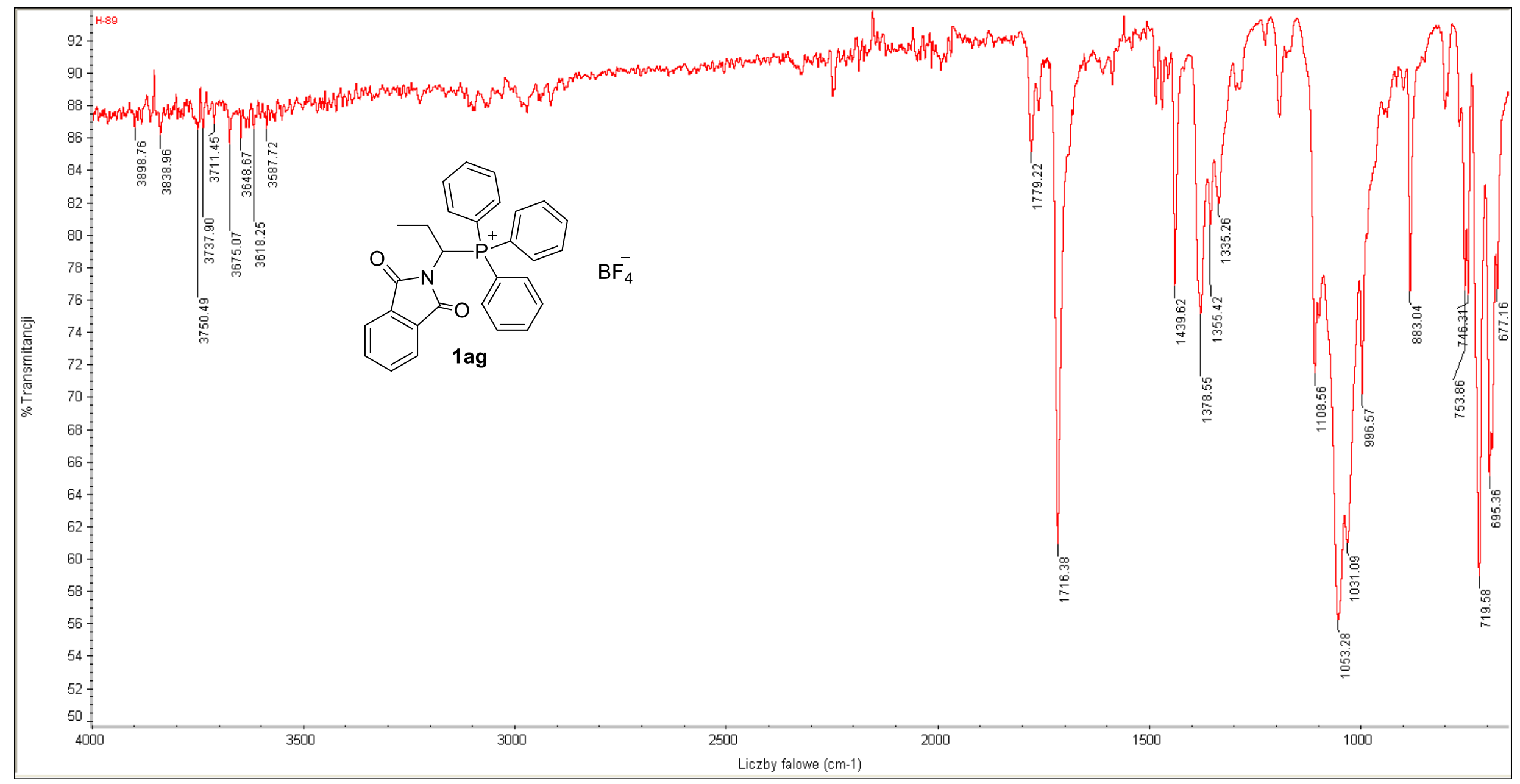

IR spectrum of 1-( $N$-phthalimido)propyltriphenylphosphonium tetrafluoroborate (1ag); ATR $\left(\mathrm{cm}^{-1}\right)$. 


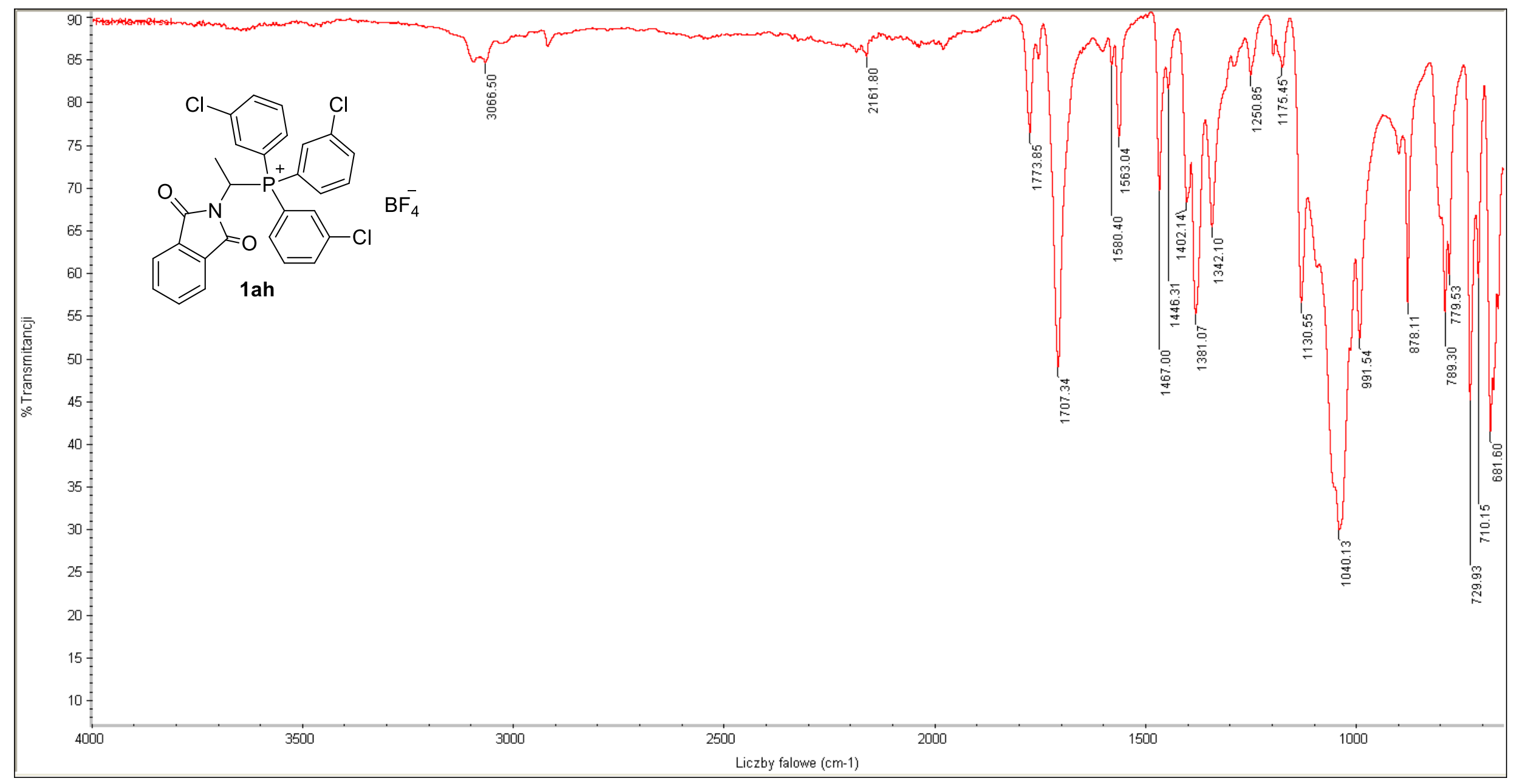

IR spectrum of 1-( $N$-phthalimido)ethyltris(3-chlorophenyl)phosphonium tetrafluoroborate (1ah); ATR $\left(\mathrm{cm}^{-1}\right)$. 


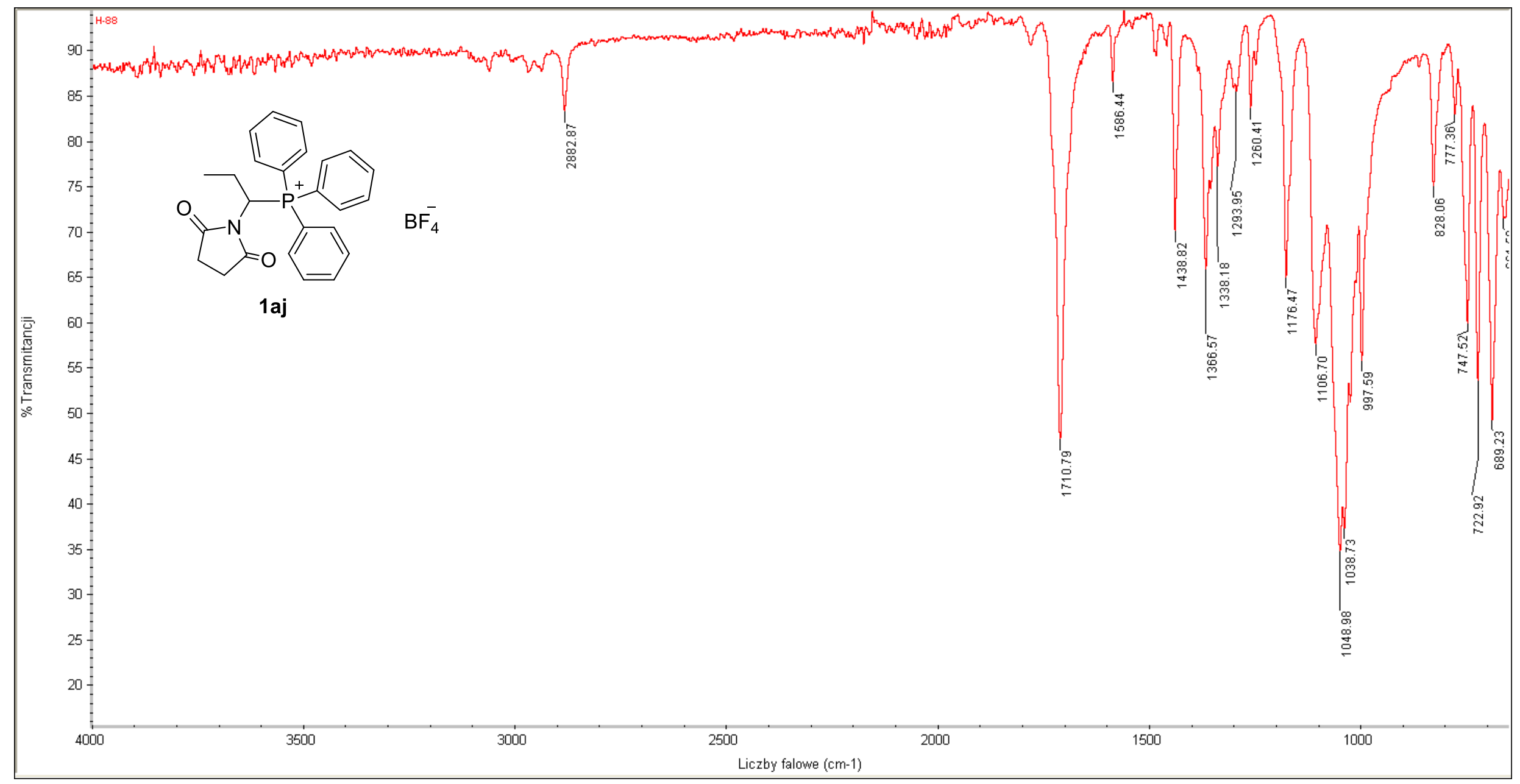

IR spectrum of 1-( $N$-succinimido)propyltriphenylphosphonium tetrafluoroborate (1aj); ATR $\left(\mathrm{cm}^{-1}\right)$. 


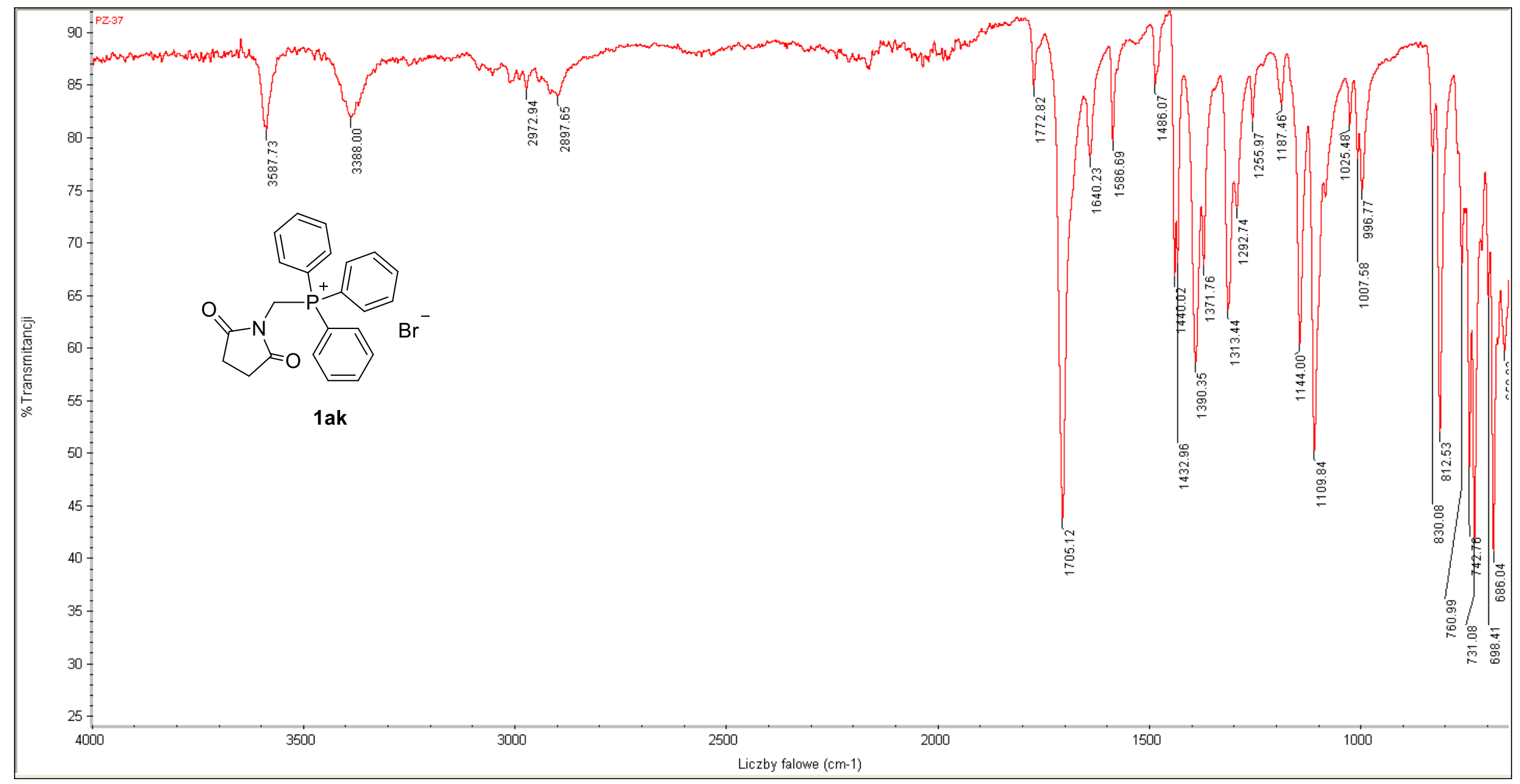

IR spectrum of 1-( $N$-succinimido)methyltriphenylphosphonium bromide (1ak); ATR $\left(\mathrm{cm}^{-1}\right)$. 


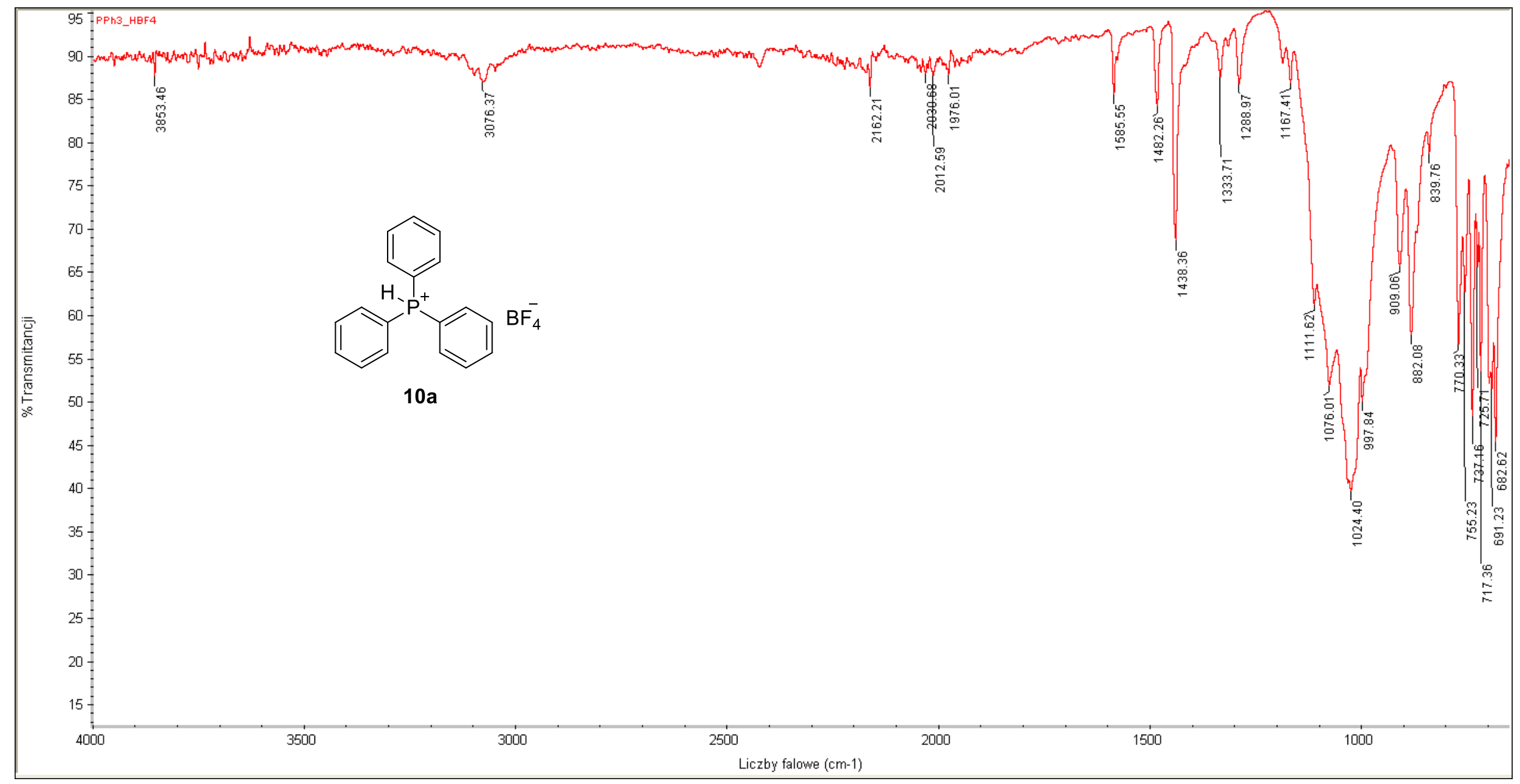

IR spectrum of triphenylphosphonium tetrafluoroborate (10a); ATR $\left(\mathrm{cm}^{-1}\right)$. 


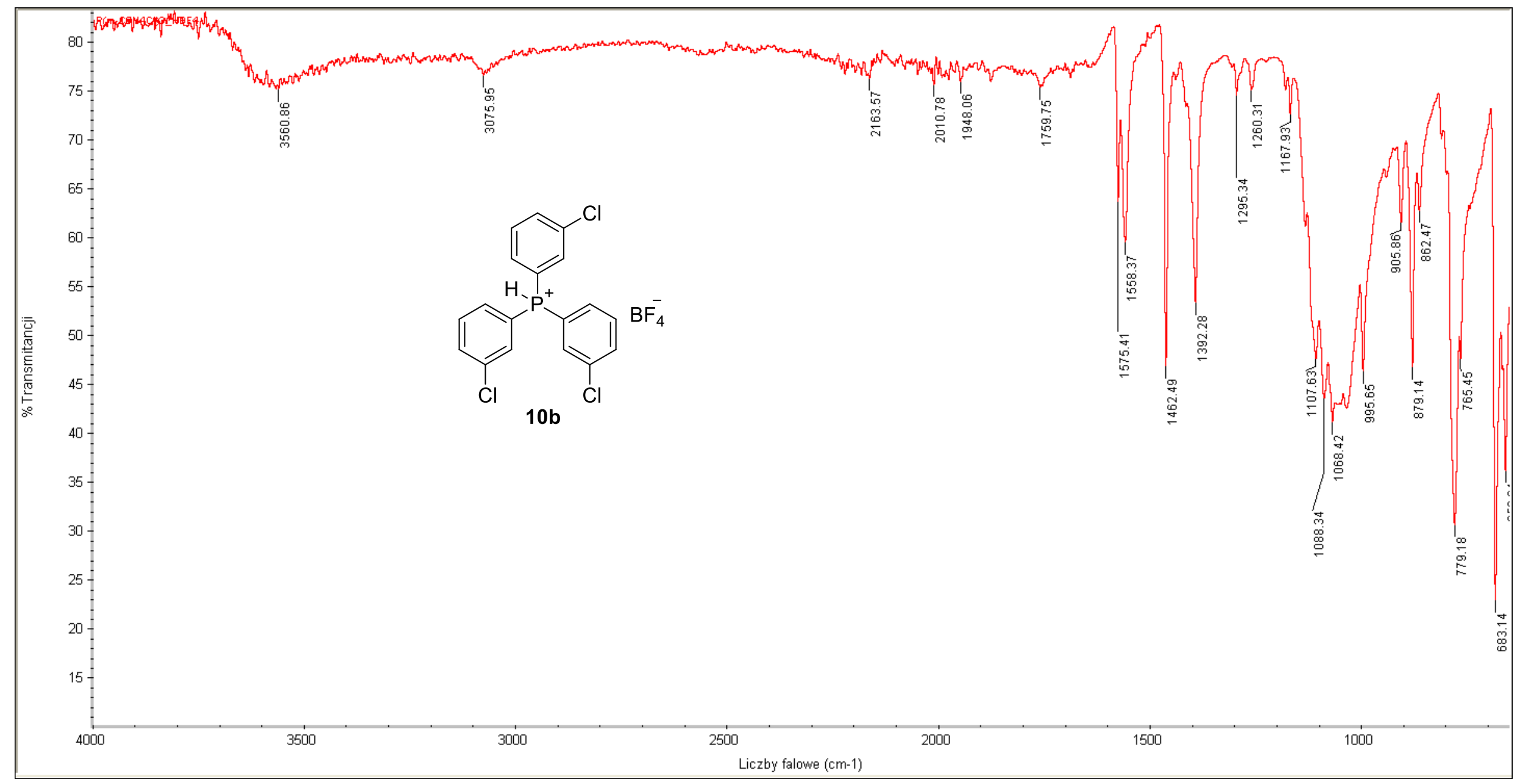

IR spectrum of tris(3-chlorophenyl)phosphonium tetrafluoroborate $(\mathbf{1 0 b})$; ATR $\left(\mathrm{cm}^{-1}\right)$. 


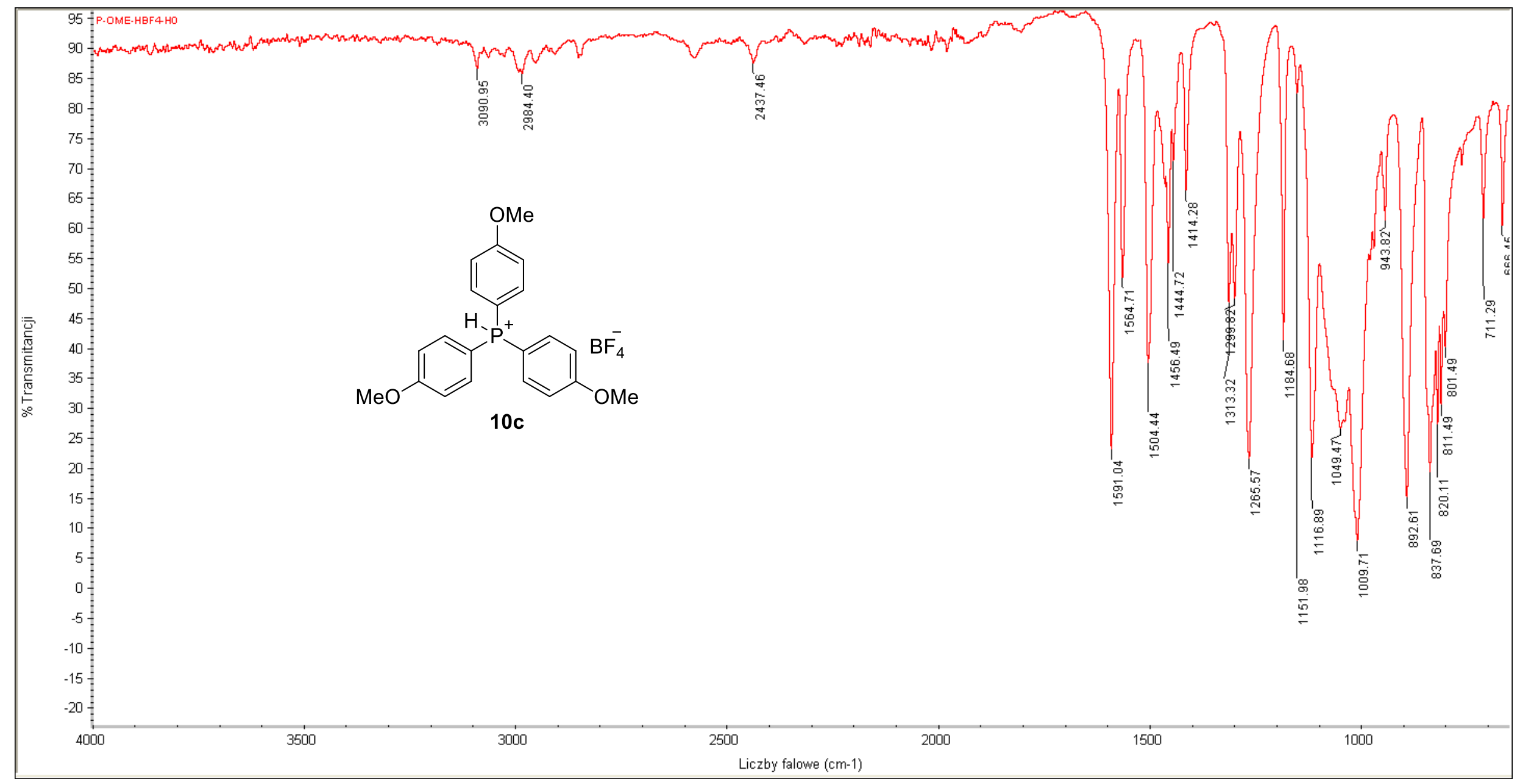

IR spectrum of tris(4-methoxyphenyl)phosphonium tetrafluoroborate (10c); ATR $\left(\mathrm{cm}^{-1}\right)$. 


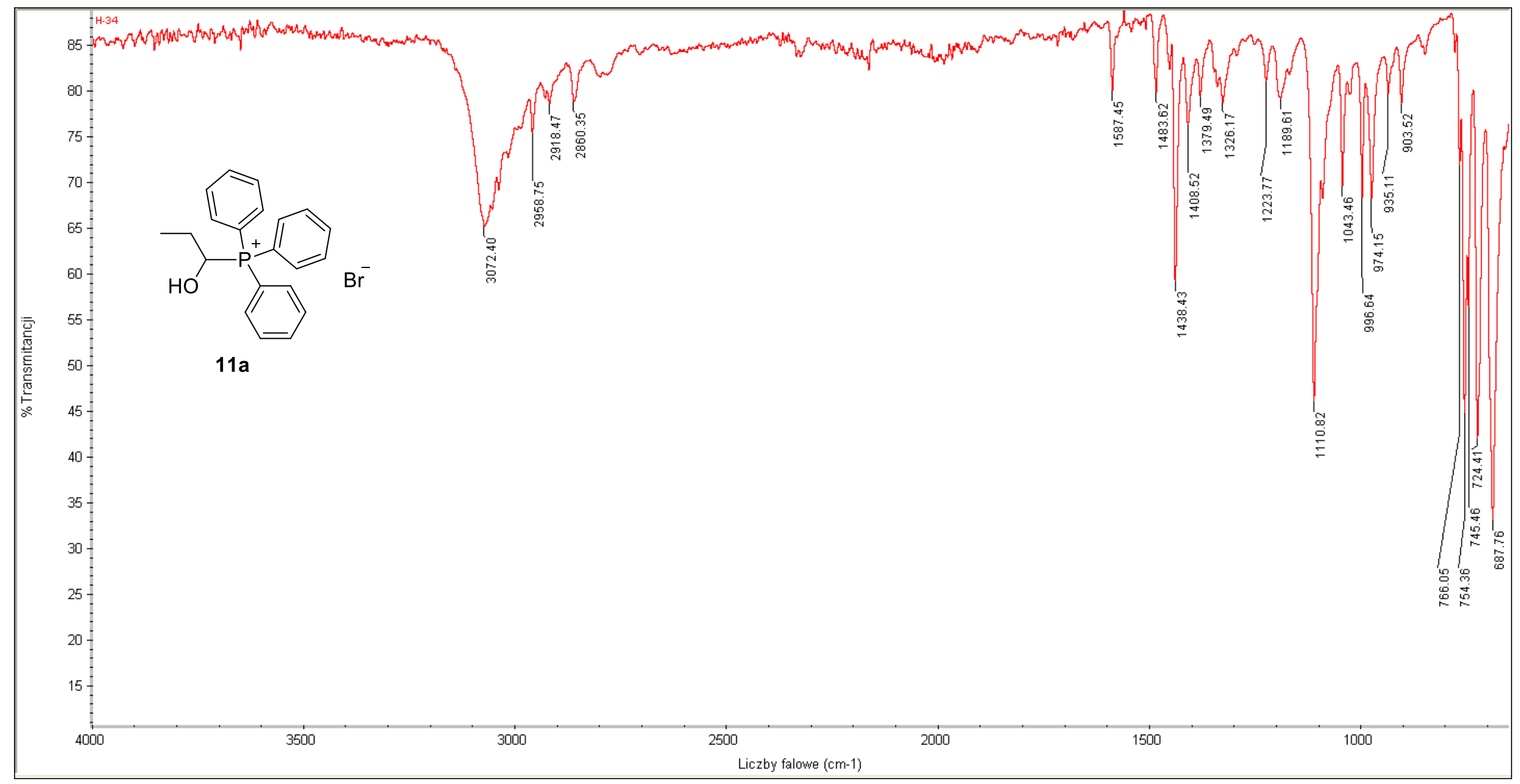

IR spectrum of 1-hydroxypropyltriphenylphosphonium bromide (11a); ATR $\left(\mathrm{cm}^{-1}\right)$. 


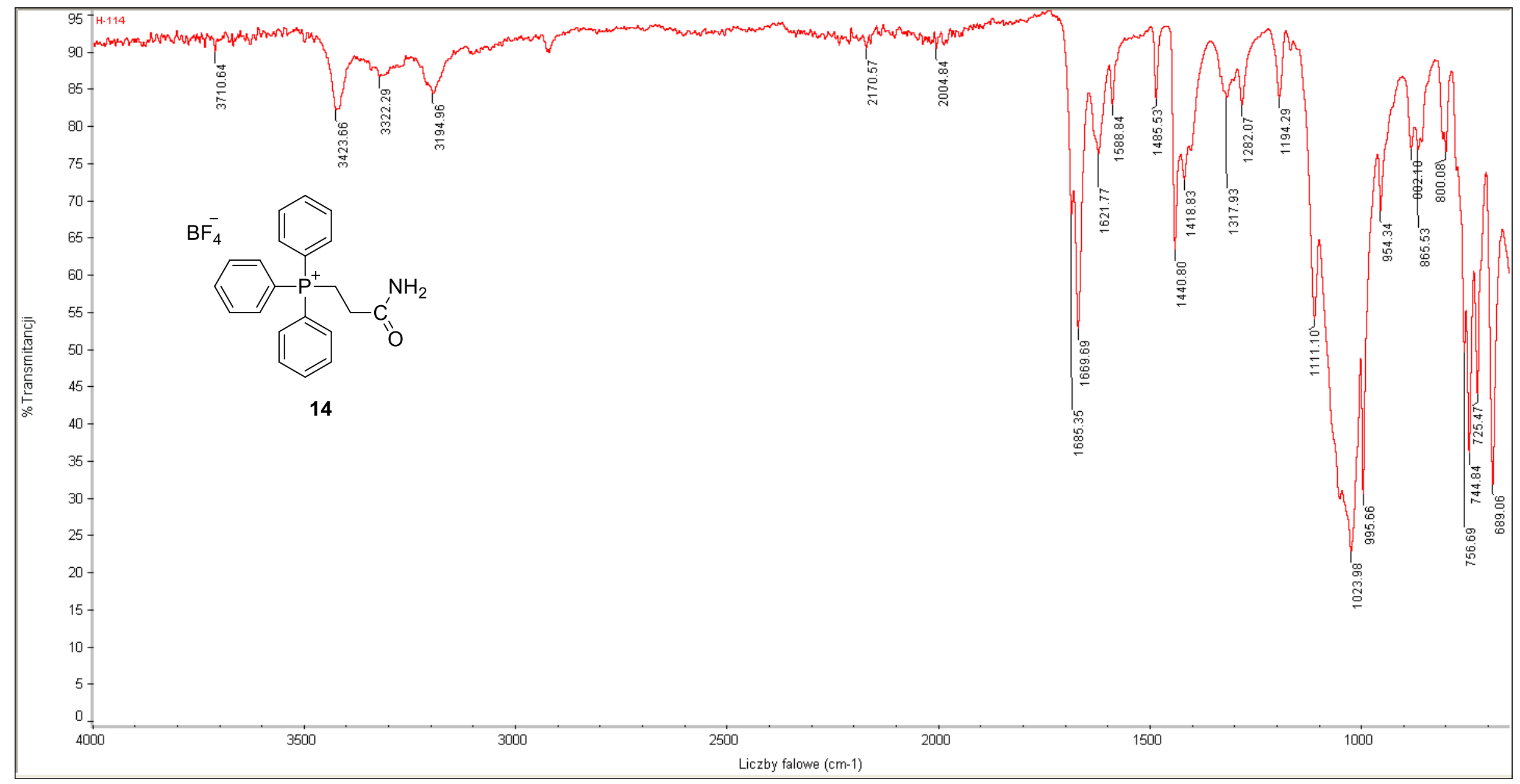

IR spectrum of 2-carbamoyletyhyltriphenylphosphonium tetrafluoroborate (14); ATR $\left(\mathrm{cm}^{-1}\right)$. 


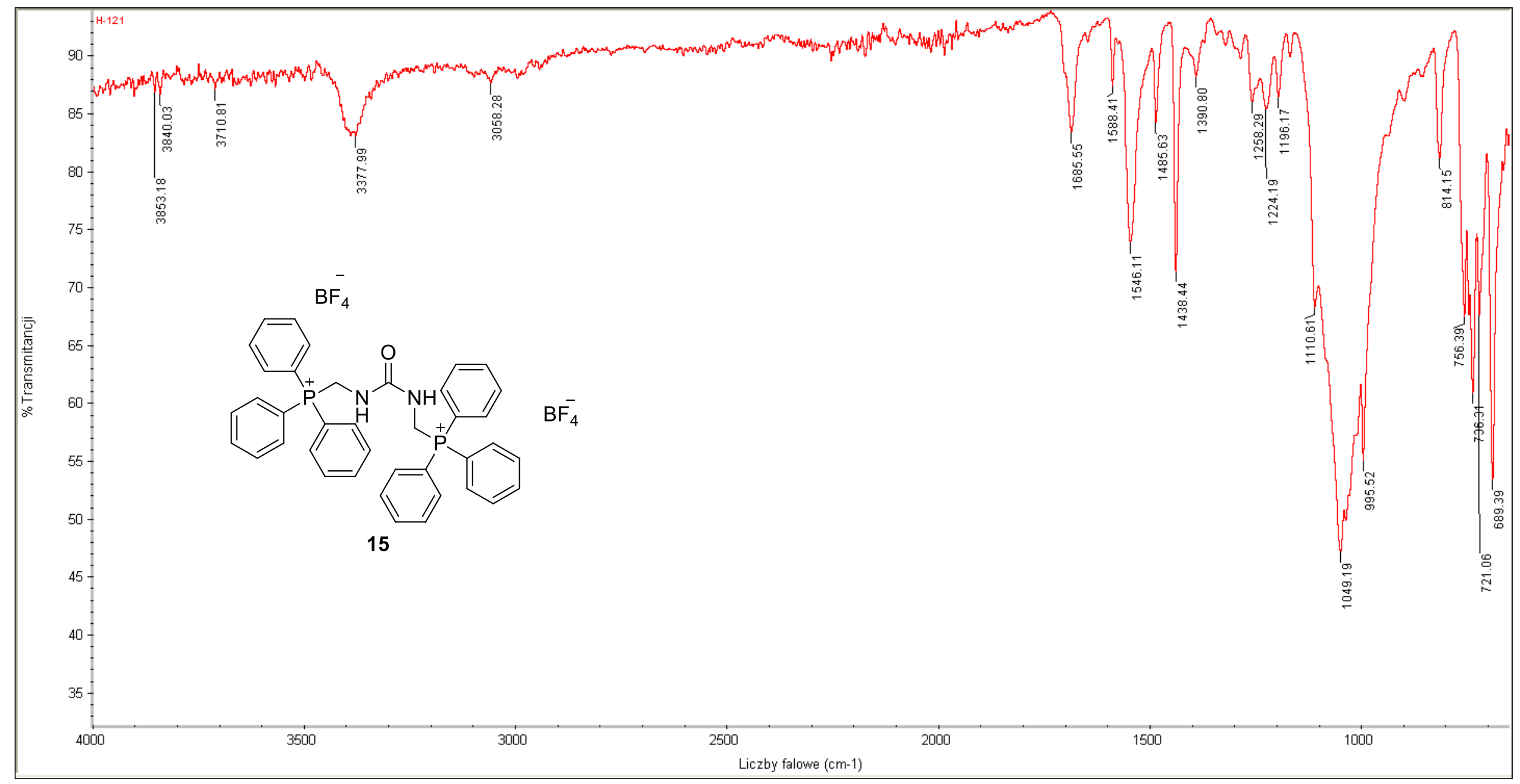

IR spectrum of 1,1'-(carbonyldimino)bis(methyltriphenylphosphonium) bis(tetrafluoroborate) (15); ATR $\left(\mathrm{cm}^{-1}\right)$. 


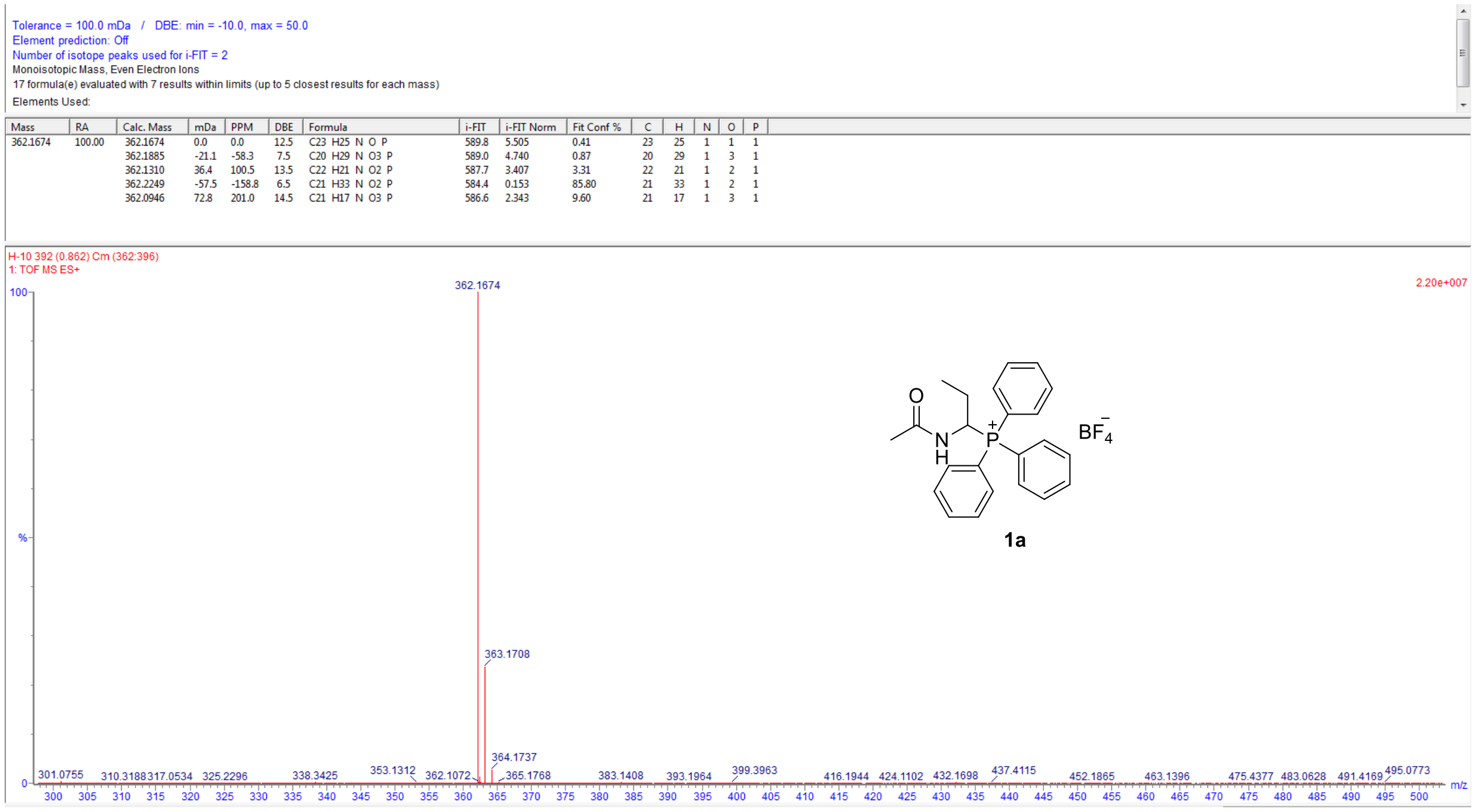

MS spectrum of 1-( $N$-acetylamino)propyltriphenylphosphonium tetrafluoroborate (1a). 


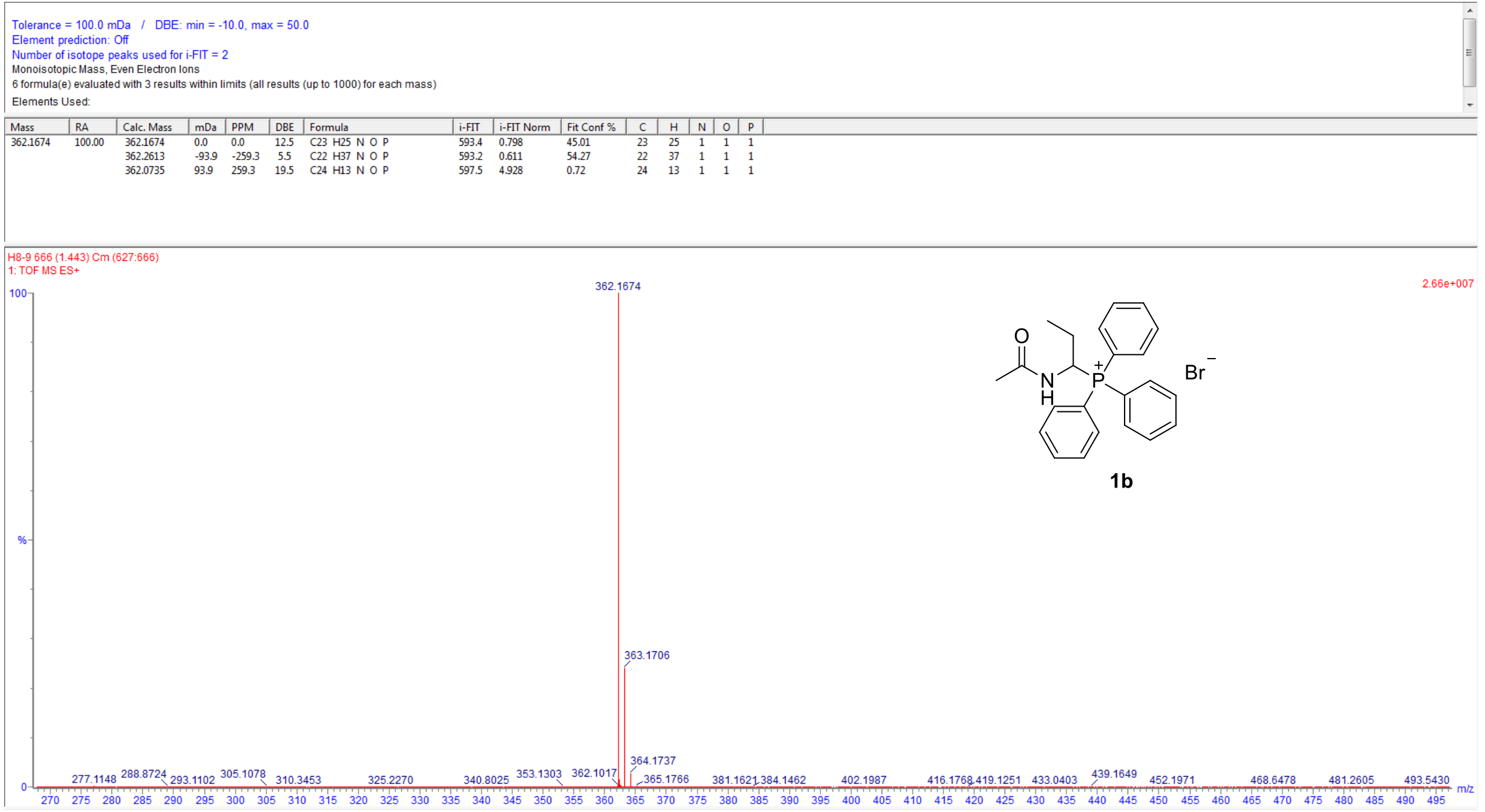

MS spectrum of 1-( $N$-acetylamino)propyltriphenylphosphonium bromide (1b). 


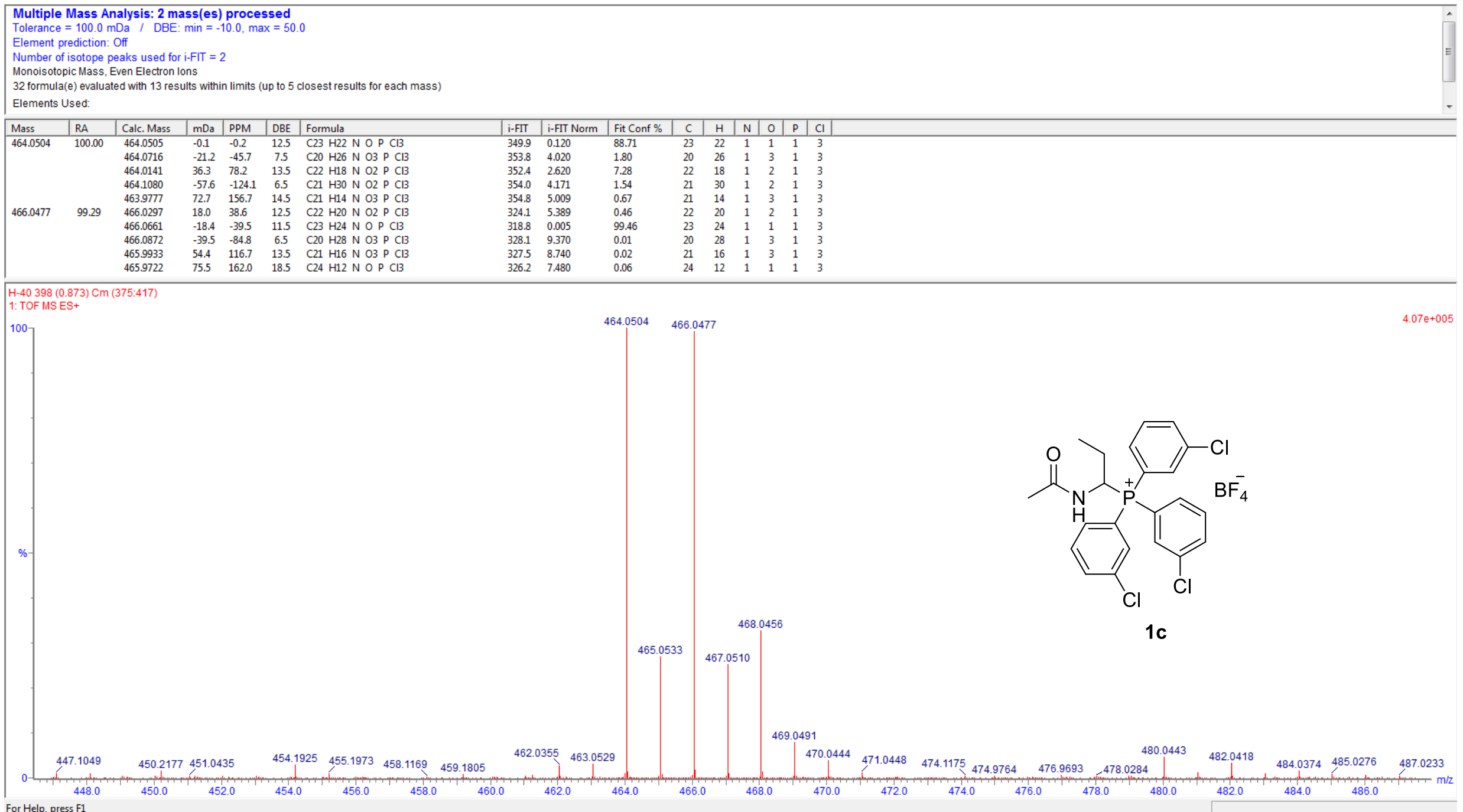

For Help, press F1

MS spectrum of 1-( $N$-acetylamino)propyltris(3-chlorophenyl)phosphonium tetrafluoroborate (1c). 


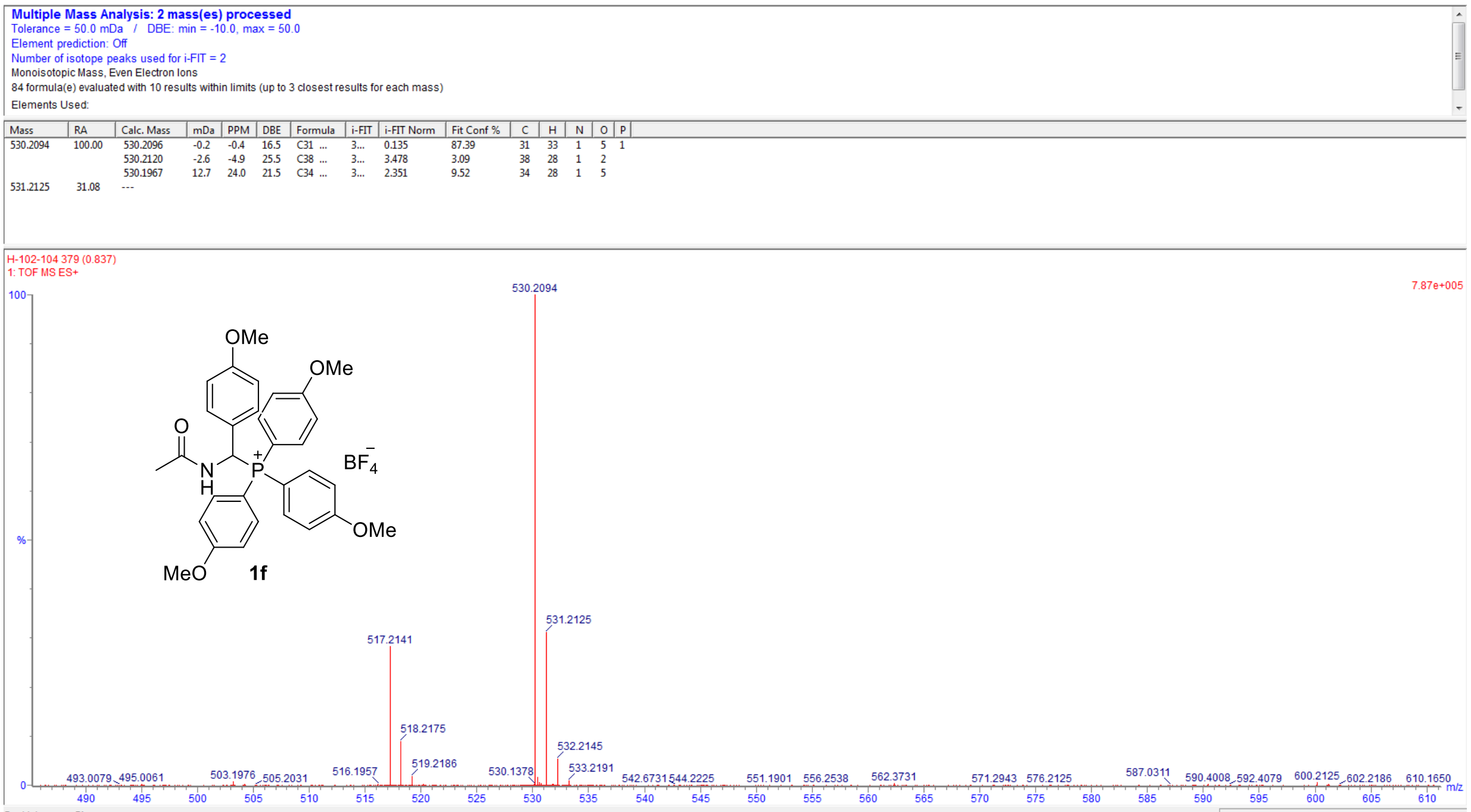

MS spectrum of 1-(N-acetylamino)-1-(4-metoxyphenyl)methyltris(4-metoxyphenyl)phosphonium tetrafluoroborate (1f). 


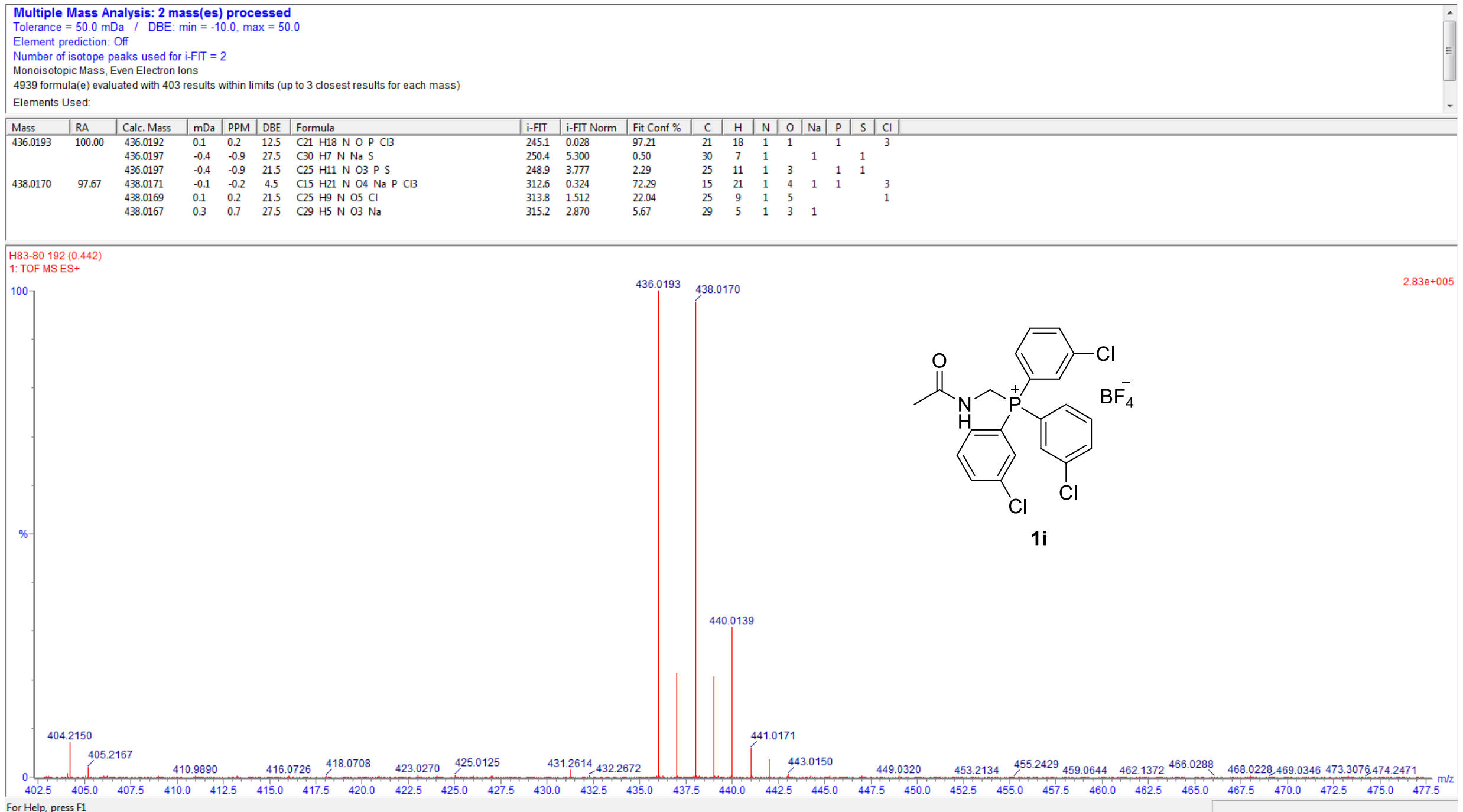

MS spectrum of ( $N$-acetylamino)methyltris(3-chlorophenyl)phosphonium tetrafluoroborate (1i). 
Tolerance $=100.0 \mathrm{mDa} /$ DBE: $\min =-10.0, \max =50.0$ Element prediction: Of

Number of isotope peaks used for $\mathrm{i}-\mathrm{FIT}=$

Monoisotopic Mass, Even Electron lons
18 formula(e) evaluated with 7 results within limits (up to 5 closest results for each mass)

Elements Usec:

\begin{tabular}{l|l}
\hline Mass & RA \\
\hline 424.1831 & 100.00
\end{tabular}

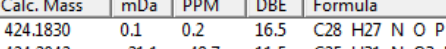

\begin{tabular}{llllll}
424.2042 & -21.1 & -49.7 & 11.5 & $\mathrm{C} 25 \mathrm{H} 31 \mathrm{~N} 03$ \\
\hline
\end{tabular}

\begin{tabular}{llllll}
424.1466 & 36.5 & 86.0 & 17.5 & $C 27$ & $\mathrm{H} 23 \mathrm{~N} 02$ \\
\hline
\end{tabular}

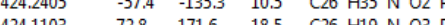

$\begin{array}{lll}533.1 & 5.505 & 0.41 \\ 527.8 & 0.233 & 79.1 \\ 532.2 & 4.580 & 1.03 \\ 529.9 & 2.323 & 9.80 \\ 529.9 & 2.345 & 9.59\end{array}$

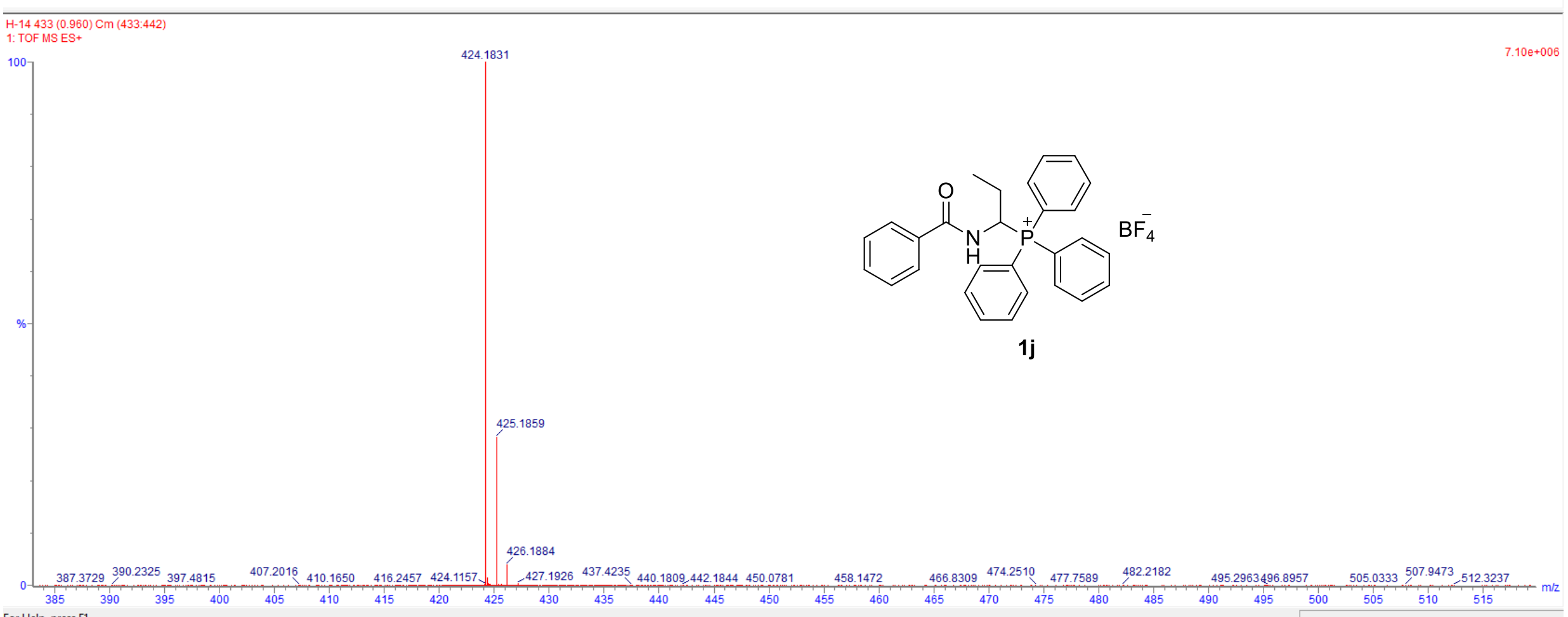

MS spectrum of 1-( $N$-benzoylamino)propyltriphenylphosphonium tetrafluoroborate (1j). 


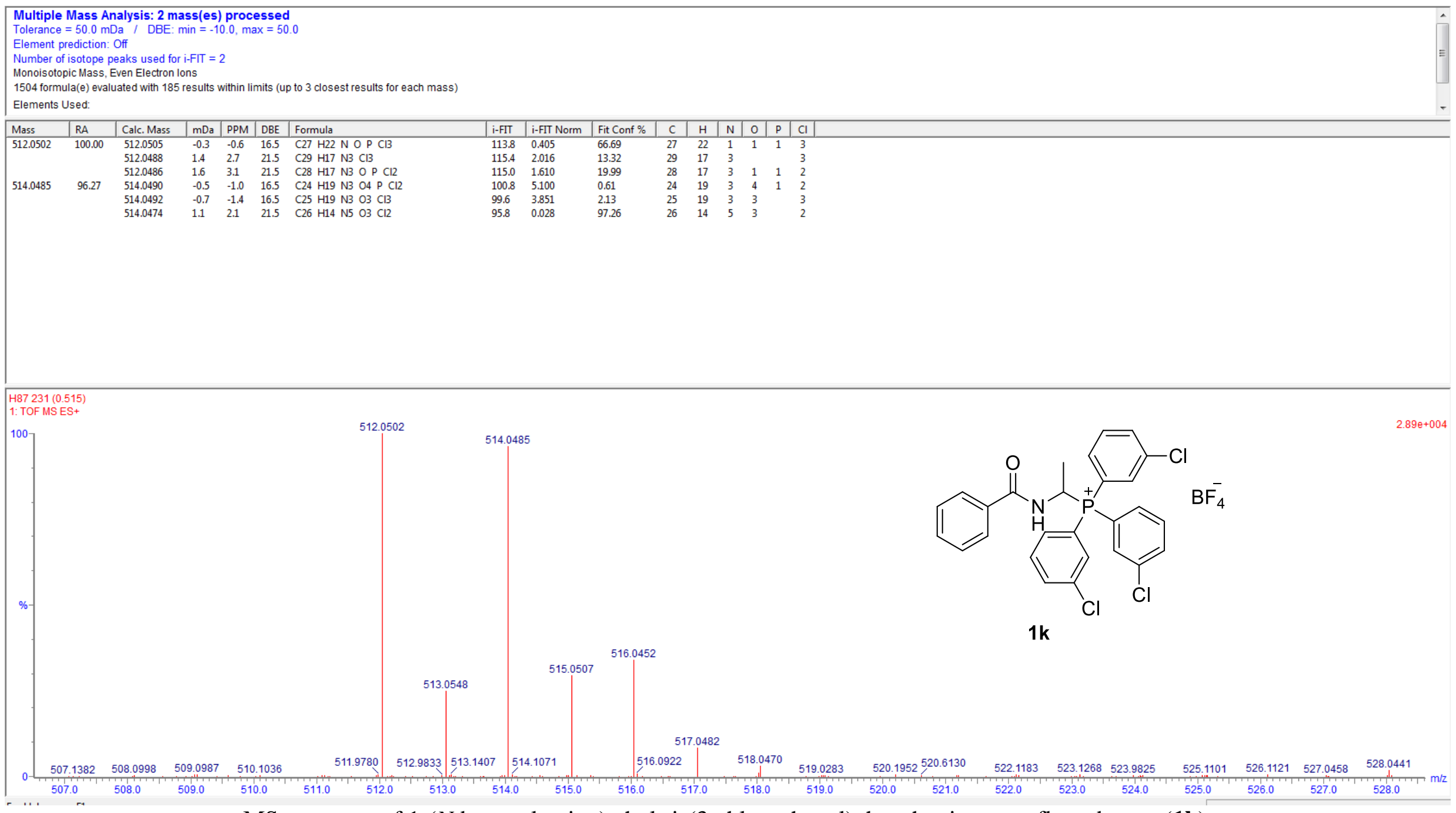

MS spectrum of 1-( $N$-benzoylamino)ethyltris(3-chlorophenyl)phosphonium tetrafluoroborate (1k). 


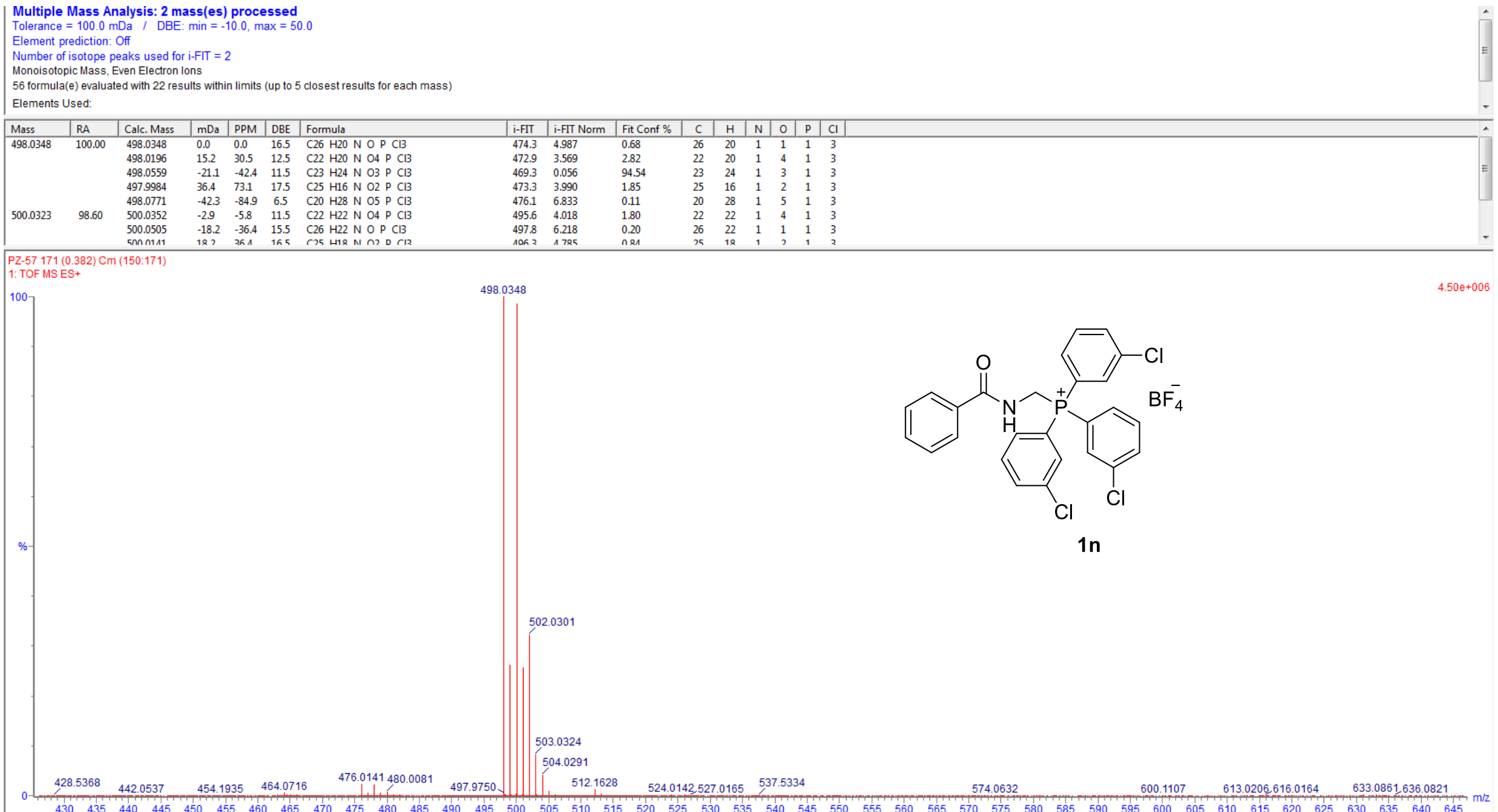

MS spectrum of ( $N$-benzoylamino)methyltris(3-chlorophenyl)phosphonium tetrafluoroborate (1n). 
Tolerance $=50.0 \mathrm{mDa} / \mathrm{DBE}: \min =-10.0, \max =50.0$

Element prediction: Off

Number of isotope peaks used for i-FIT $=2$

Monoisotopic Mass, Even Electron lons

within limits (up to 3 closest results for each mas

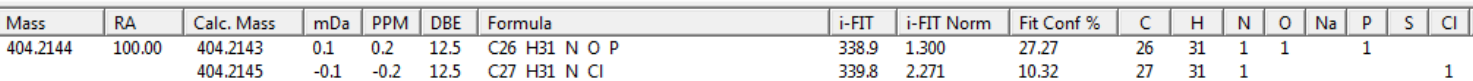

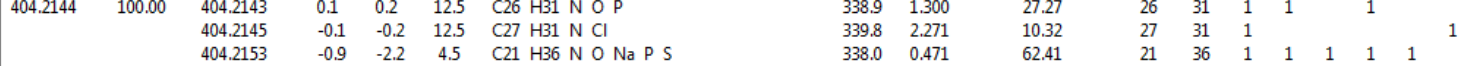

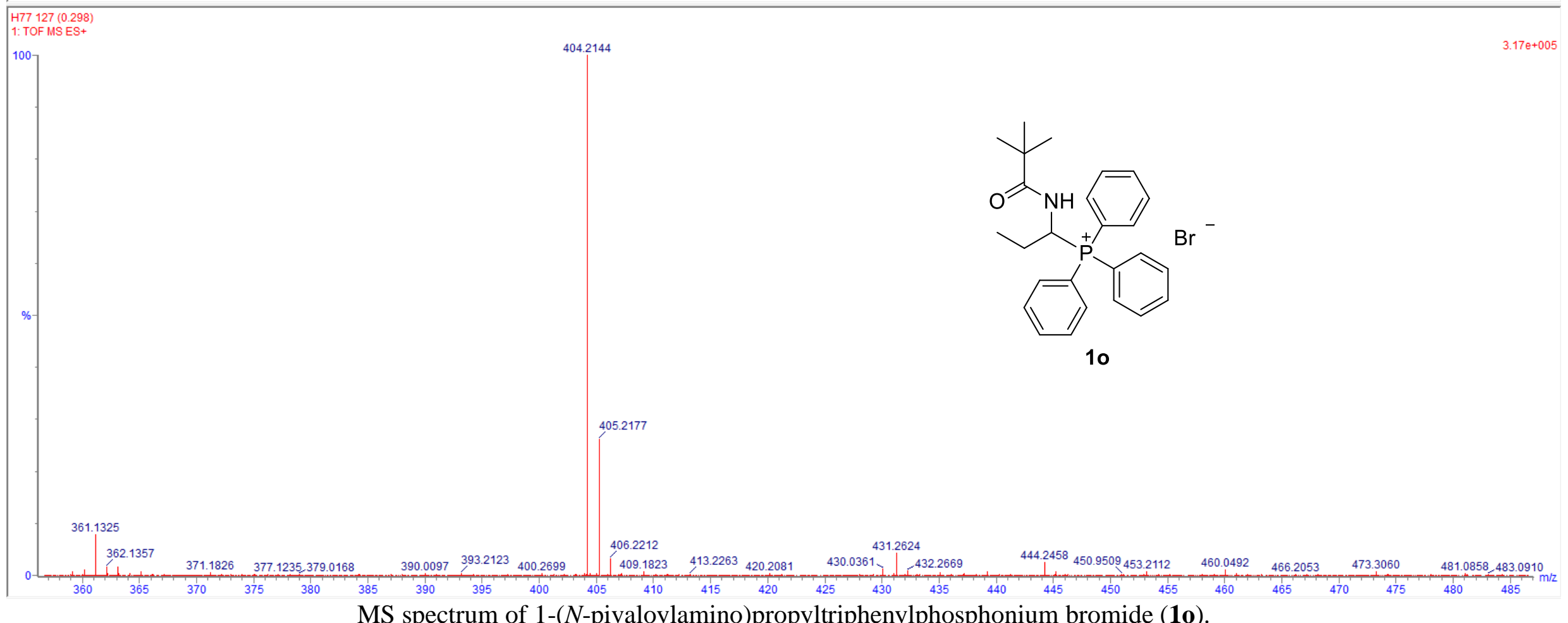

MS spectrum of 1-( $N$-pivaloylamino)propyltriphenylphosphonium bromide (1o). 
Tolerance $=50.0 \mathrm{mDa} /$ DBE: $\min =-10.0, \max =50.0$ Element prediction: $\mathrm{O}$

Number of isotope peaks used for i-FIT $=2$

Elements Used:

\begin{tabular}{|c|c|c|c|c|c|c|c|c|c|c|c|c|c|c|c|}
\hline \multirow{3}{*}{$\begin{array}{l}\text { Mass } \\
458.1707\end{array}$} & RA & Calc. Mass & $m D_{a}$ & PPM & DBE & Formula & i-FIT & i-FIT Norm & Fit Conf $\%$ & C & $\mathrm{H}$ & $\mathrm{N}$ & & $\mathrm{Na}$ & \\
\hline & 100.00 & 458.1707 & 0.0 & 0.0 & 15.5 & $\mathrm{C} 28 \mathrm{H} 29 \mathrm{~N} O \mathrm{PS}$ & 468.7 & 1.034 & 35.55 & 28 & 29 & 1 & 1 & & $\begin{array}{lll}1 & 1 \\
1 & 1\end{array}$ \\
\hline & & $\begin{array}{l}458.1709 \\
458.1710\end{array}$ & $\begin{array}{l}-0.2 \\
-0.3\end{array}$ & $\begin{array}{l}-0.4 \\
-0.7\end{array}$ & $\begin{array}{c}15.5 \\
8.5\end{array}$ & $\begin{array}{l}\mathrm{C} 29 \mathrm{H} 29 \mathrm{~N} \mathrm{~S} \mathrm{Cl} \\
\mathrm{C} 23 \mathrm{H} 30 \mathrm{~N} 05 \mathrm{Na} \mathrm{Cl}\end{array}$ & $\begin{array}{l}468.2 \\
470.9\end{array}$ & $\begin{array}{l}0.506 \\
3.177\end{array}$ & $\begin{array}{l}60.28 \\
4.17\end{array}$ & $\begin{array}{l}29 \\
23\end{array}$ & $\begin{array}{l}29 \\
30\end{array}$ & $\begin{array}{l}1 \\
1\end{array}$ & 5 & 1 & \\
\hline
\end{tabular}

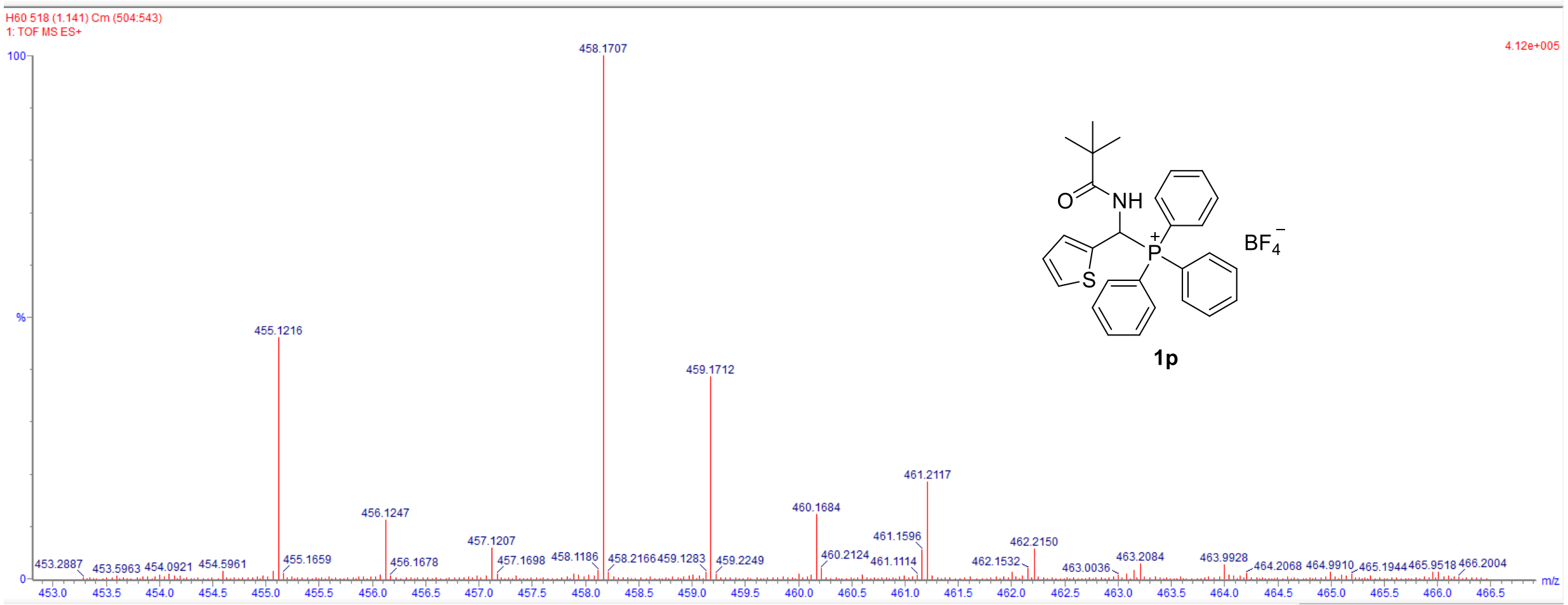

MS spectrum of 1-(N-pivaloylamino)-1-(2-thienyl)methyltriphenylphosphonium tetrafluoroborate (1p). 


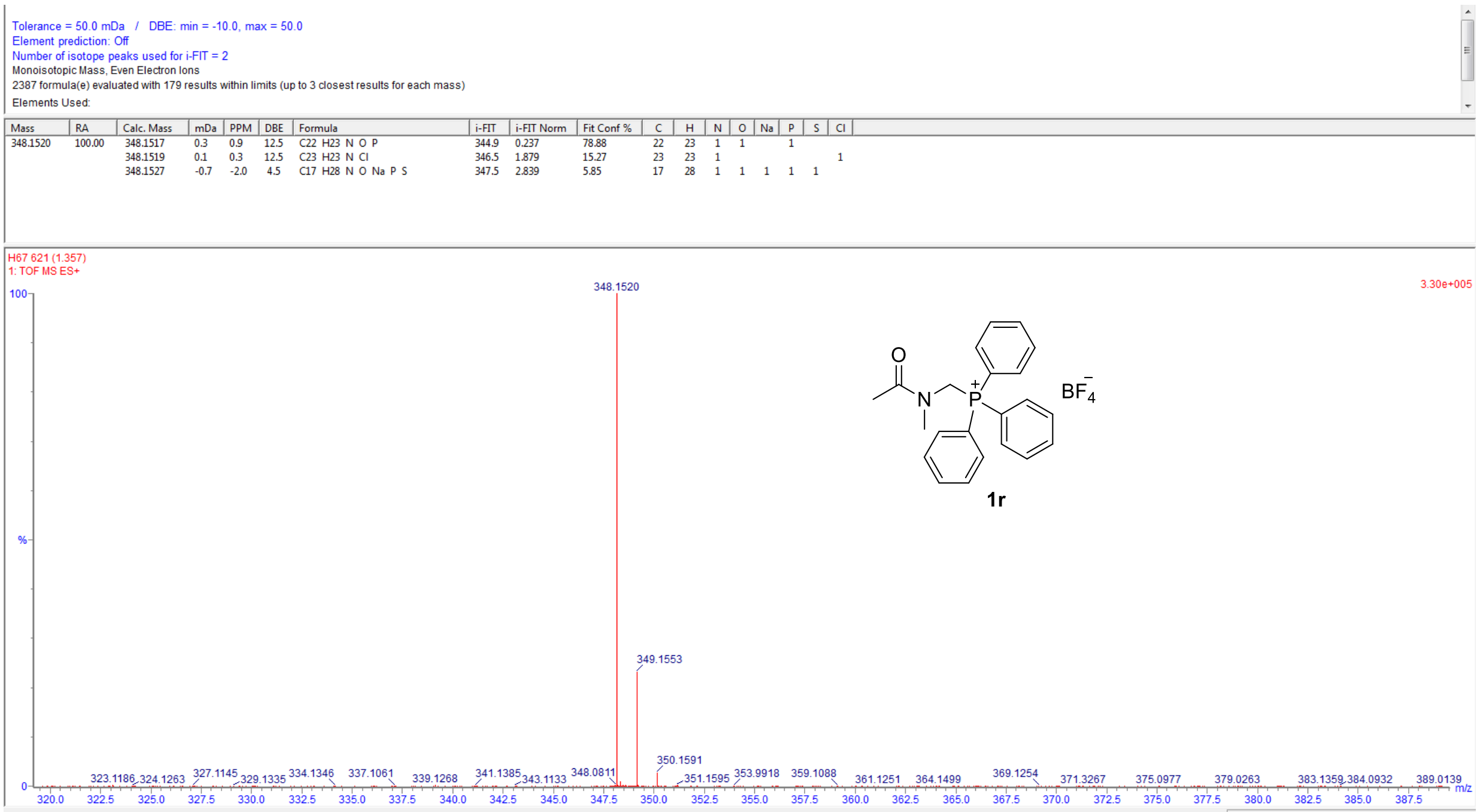

MS spectrum of $N$-(N-methylacetylamino)methyltriphenylphosphonium tetrafluoroborate (1r). 


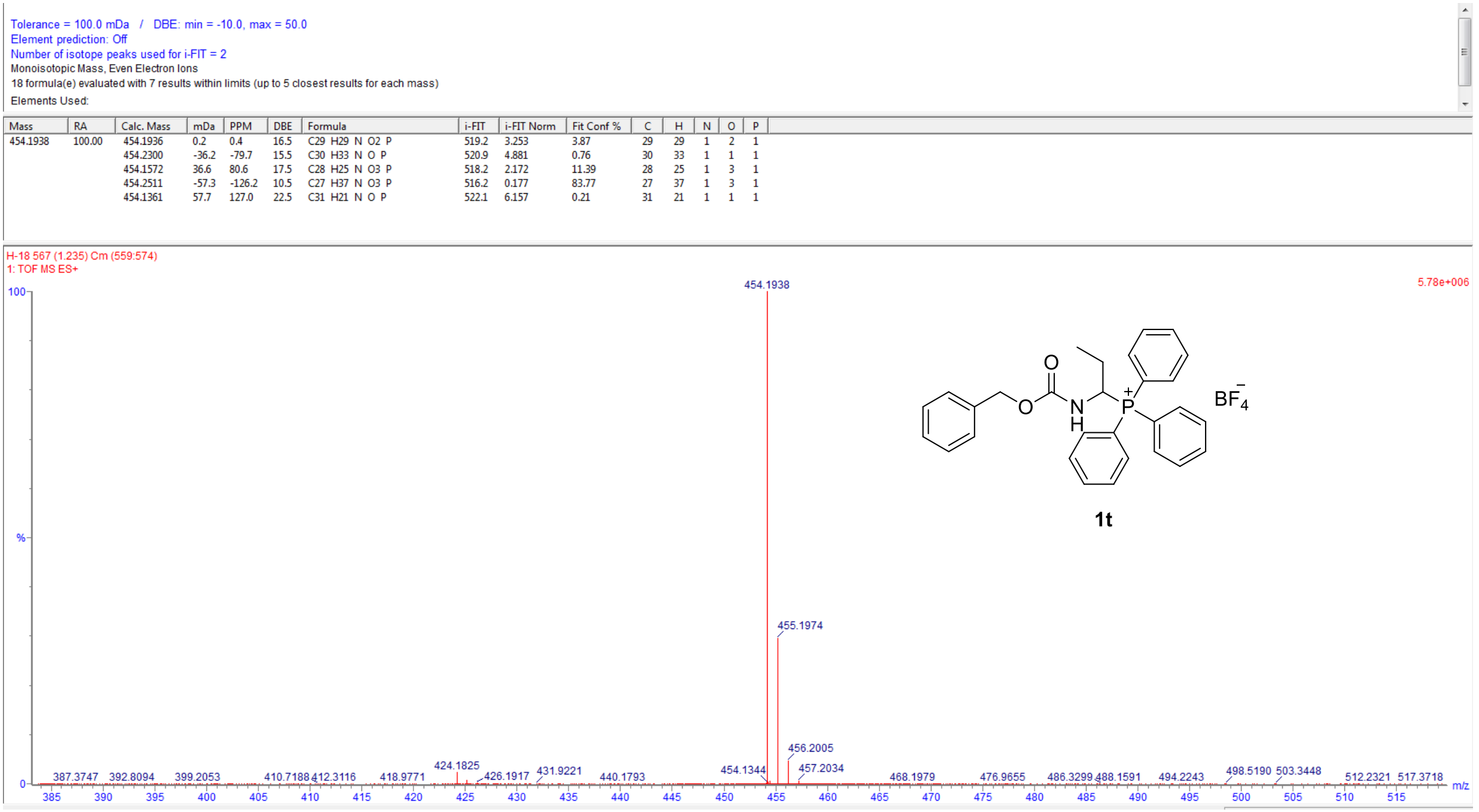

MS spectrum of 1-( $N$-benzyloxycarbonylamino)propyltriphenylphosphonium tetrafluoroborate (1t). 
Element predictin

Number of isotope peaks used for i-FIT $=2$

26 formula(e) evaluated with 3 results within limits (up to 3 closest results for each mass)

Elements Used:

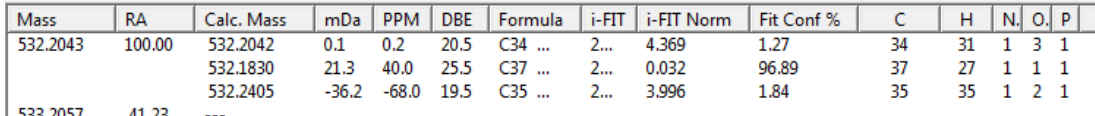

533.2057

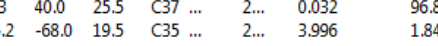

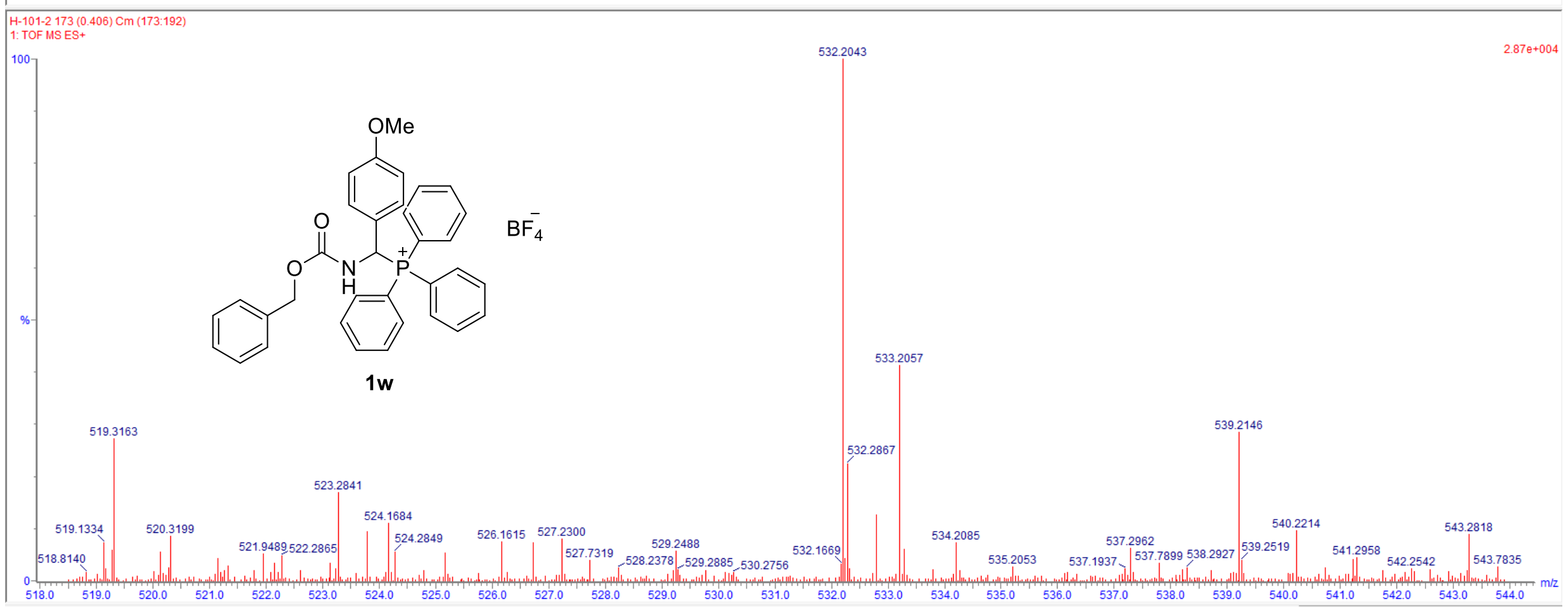

MS spectrum of 1-(N-benzyloxycarbonylamino)-1-(4-methoxyphenyl)methyltriphenylphosphonium tetrafluoroborate (1w). 
Number of isotope peaks used for $\mathrm{i}-\mathrm{FIT}=2$

Monoisotopic Mass, Even Electron lons

68 formula(e) evaluated with 4 results within limits (up to 3 closest results for each mass)

Elements Used:

\begin{tabular}{|c|c|c|c|c|c|c|c|c|c|c|c|c|c|}
\hline & RA & Calc. Mass & $\mathrm{mDa}$ & PPM & DBE & Formula & i-FIT & i-FIT Norm & Fit Conf \% & C & $\mathrm{H}$ & & \\
\hline & 100.00 & 547.1787 & -0.3 & -0.5 & 21.5 & $\mathrm{C} 33 \mathrm{H} 28 \mathrm{~N} 2 \mathrm{O} 4 \mathrm{P}$ & 329.7 & 0.006 & 99.40 & 33 & 28 & 2 & 4 \\
\hline \multirow{2}{*}{$\begin{array}{l}547.1784 \\
548.1815\end{array}$} & & 547.1939 & -15.5 & -28.3 & 25.5 & $\mathrm{C} 37 \mathrm{H} 28$ & 334.8 & 5.114 & 0.60 & 37 & 28 & 2 & \\
\hline & 34.60 & 548.1779 & 3.6 & 6.6 & 25.5 & $\mathrm{C} 37 \mathrm{H} 27 \mathrm{~N} 02 \mathrm{P}$ & 258.4 & 0.161 & 85.13 & 37 & 27 & 1 & 2 \\
\hline & & 548.1991 & -17.6 & -32.1 & 20.5 & C34 H31 N & 260.2 & 1.906 & 14.87 & 34 & & & \\
\hline
\end{tabular}

H-105-2 489 (1.067) Cm (481:498)
1: TOF MS ES+

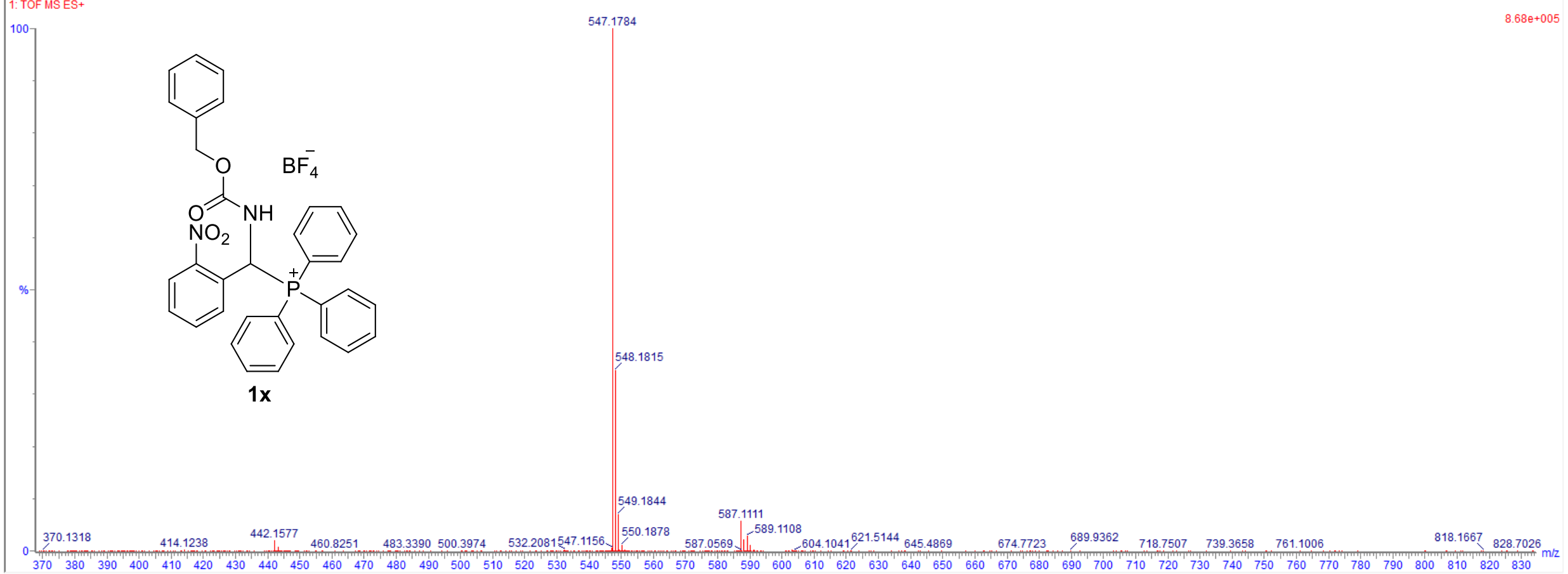

MS spectrum of 1-(N-benzyloxycarbonylamino)-1-(2-nitrophenyl)methyltriphenylphosphonium tetrafluoroborate (1x). 


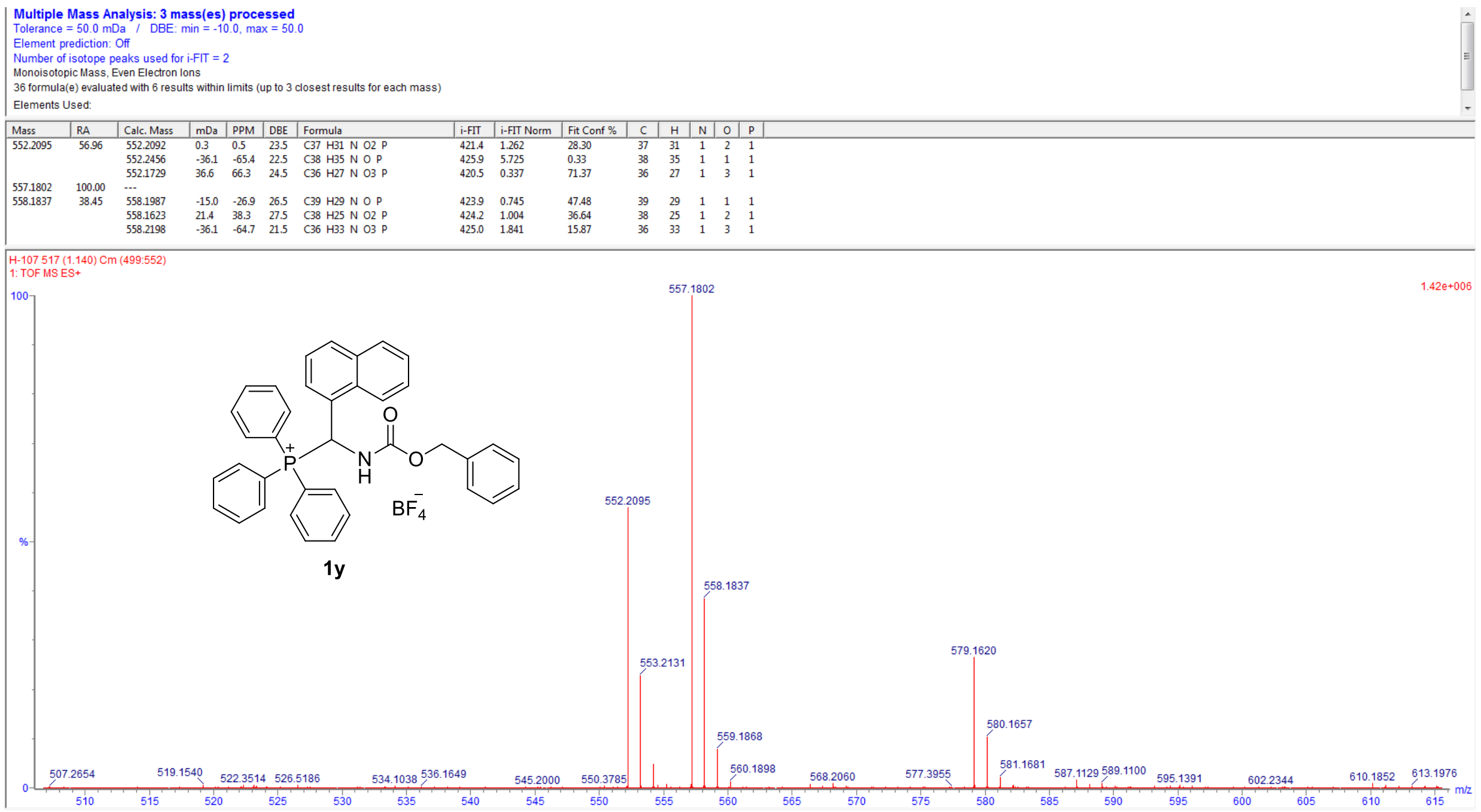

MS spectrum of 1-(N-benzyloxycarbonylamino)-1-(1-naphthyl)methyltriphenylphosphonium tetrafluoroborate (1y). 


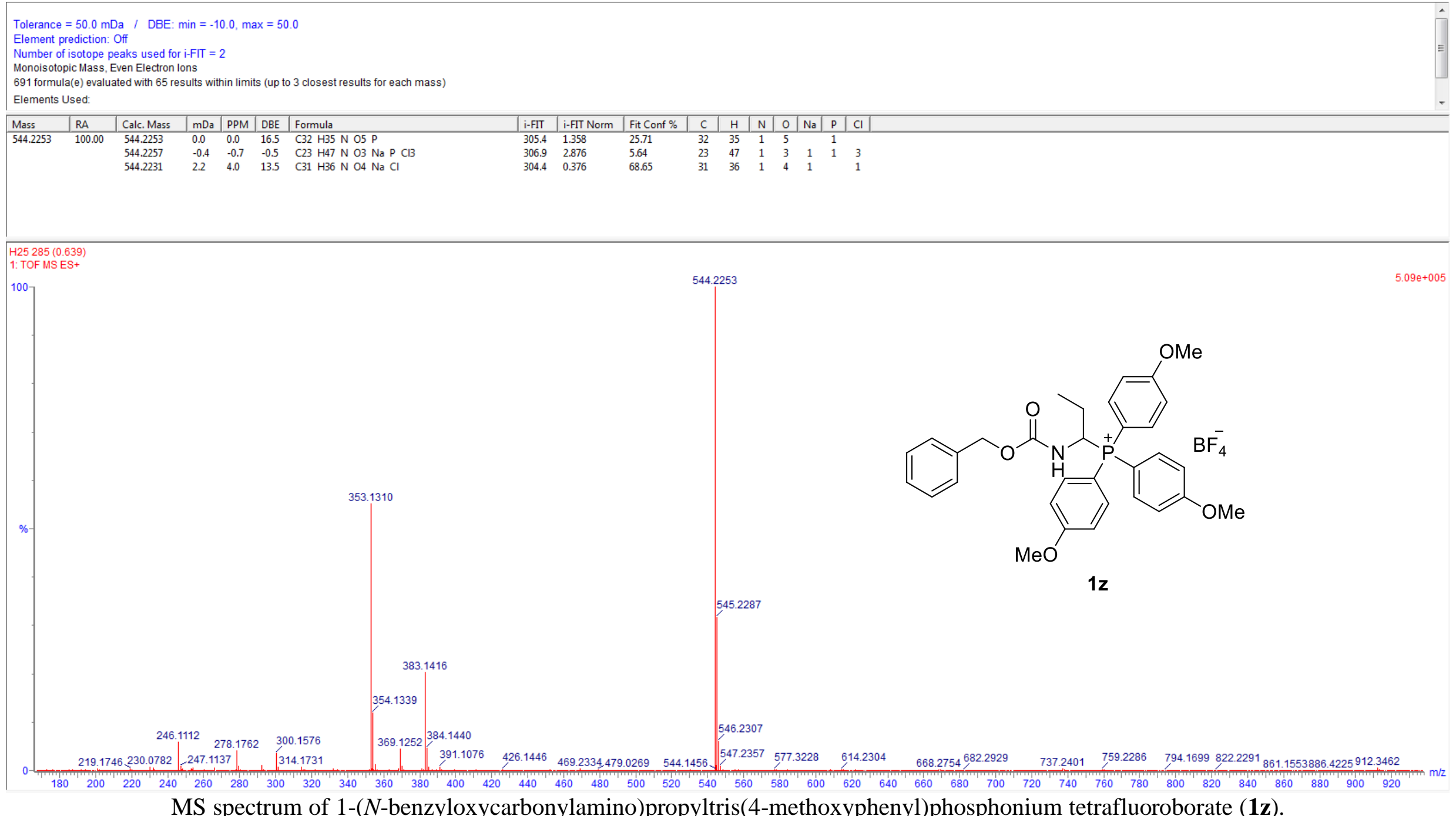

MS spectrum of 1-(N-benzyloxycarbonylamino)propyltris(4-methoxyphenyl)phosphonium tetrafluoroborate (1z). 


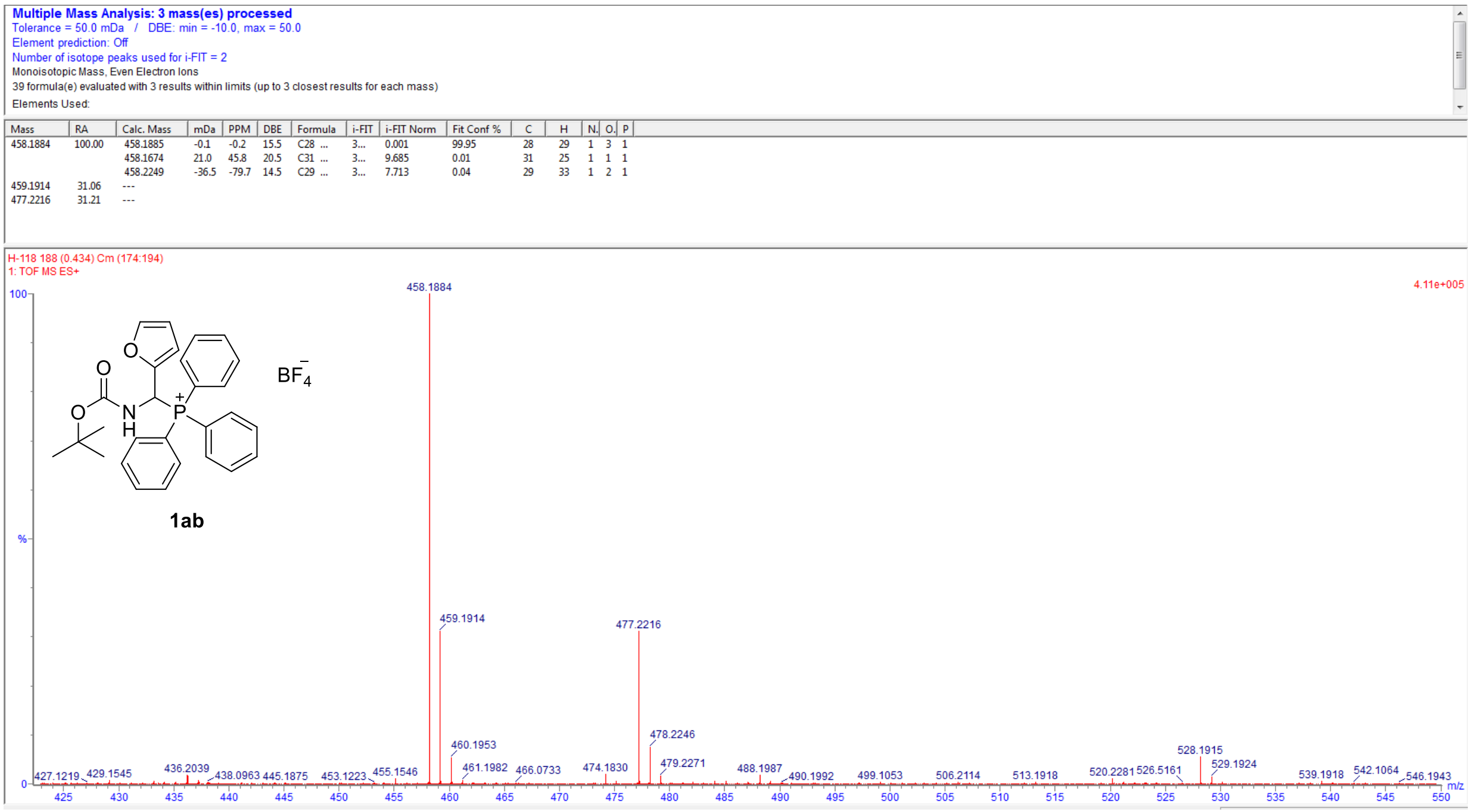

MS spectrum of 1-(N-tert-butoxycarbonylamino)-1-(2-furyl)methyltriphenylphosphonium tetrafluoroborate (1ab). 
Tolerance $=50.0 \mathrm{mDa} / \mathrm{DBE}: \min =-10.0, \max =50.0$

Tolerance $=50.0 \mathrm{mDa}$

Number of isotope peaks used for $\mathrm{i}-\mathrm{FIT}=2$

2454 formula(e) evaluated with 185 results within limits (up to 3 closest results for each mass)

Elements Used:

\begin{tabular}{|c|c|c|c|c|c|c|c|c|c|c|c|c|c|c|c|}
\hline Mass & RA & Calc. Mass & $\mathrm{mDa}$ & PPM & DBE & Formula & i-FTT & i-FTT Norm & Fit Conf $\%$ & C & H & $\mathrm{N}$ & & $\mathrm{Na}$ & \begin{tabular}{l|ll}
$\mathrm{s}$ & $\mathrm{Cl}$
\end{tabular} \\
\hline 388.1831 & 100.00 & $\begin{array}{l}388.1830 \\
388.1832\end{array}$ & $\begin{array}{l}0.1 \\
-0.1\end{array}$ & $\begin{array}{l}0.3 \\
-0.3\end{array}$ & $\begin{array}{l}13.5 \\
13.5\end{array}$ & $\begin{array}{l}\mathrm{C} 25 \mathrm{H} 27 \mathrm{~N} \mathrm{O} \mathrm{P} \\
\mathrm{C} 26 \mathrm{H} 27 \mathrm{~N} \mathrm{Cl}\end{array}$ & $\begin{array}{l}113.1 \\
115.0\end{array}$ & $\begin{array}{l}0.140 \\
2.061\end{array}$ & $\begin{array}{l}86.91 \\
12.73\end{array}$ & $\begin{array}{l}25 \\
26\end{array}$ & $\begin{array}{l}27 \\
27\end{array}$ & $\begin{array}{l}1 \\
1\end{array}$ & 1 & & \\
\hline & & $\begin{array}{l}30.08 .1840 \\
3840\end{array}$ & $\begin{array}{l}-0.1 \\
-0.9\end{array}$ & -2.3 & 5.5 & C20 H32 N O NaP S & 118.6 & 5.610 & 0.37 & 20 & 32 & 1 & 1 & 1 & 1 \\
\hline
\end{tabular}

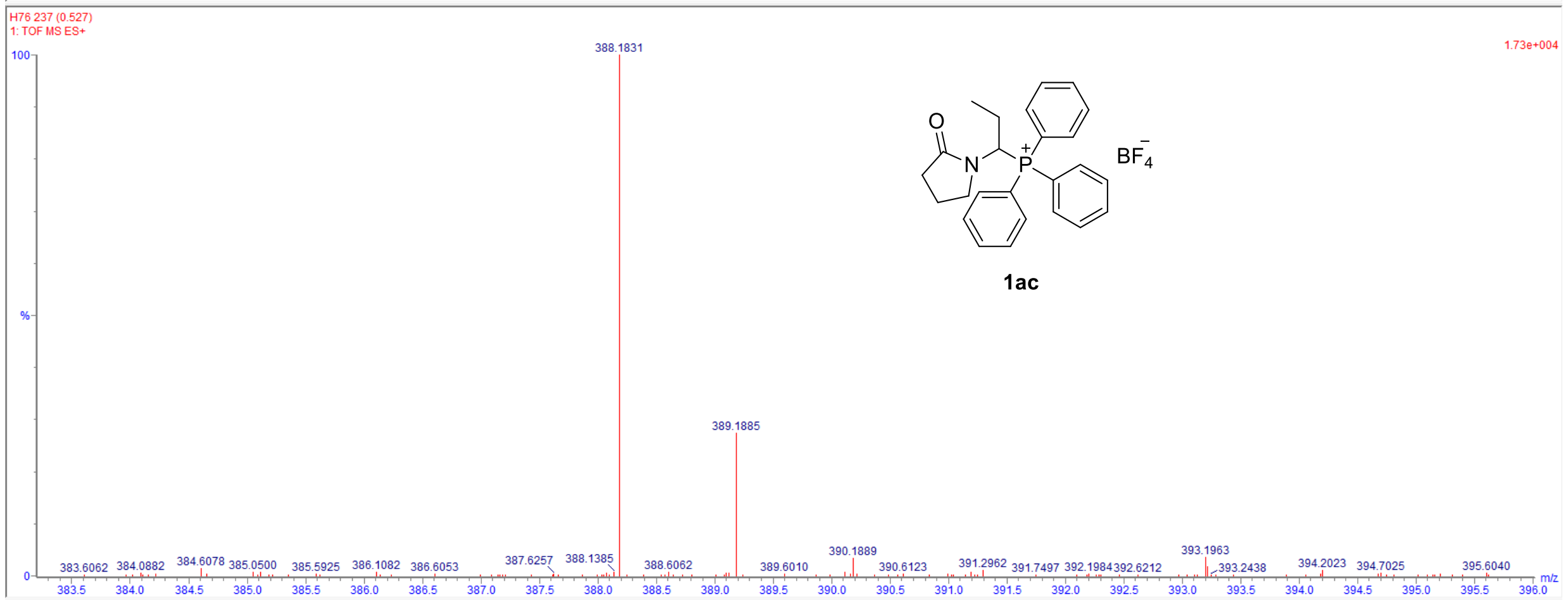

MS spectrum of 1-(2-oxopyrrolidin-1-yl)propyltriphenylphosphonium tetrafluoroborate (1ac). 
Tolerance $=50.0 \mathrm{mDa} / \mathrm{DBE}: \min =-10.0, \max =50.0$

Element prediction: $\mathrm{O}$

Number of isotope peaks used for $\mathrm{i}-\mathrm{FIT}=2$

2407 formula(e) evaluated with 185 results within limits (up to 3 closest results for each mass)

Elements Used:

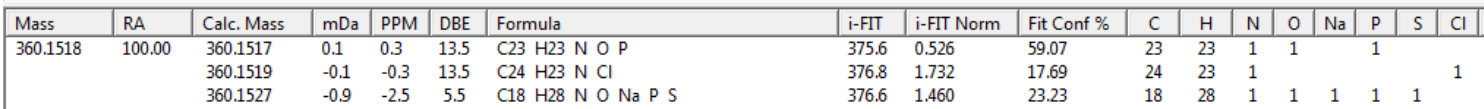

H68 156 (0.354)
1: TOF MS ES+

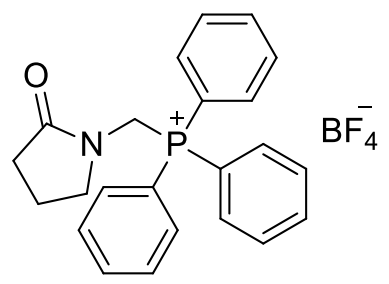

1ad

$\%-$

343.1139345 .0834

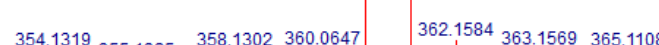

$\begin{array}{lll}369.2486 & 372.3483 & 374.1678 \\ 375.1688\end{array}$

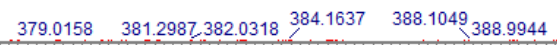
$\frac{392.1425}{39} \mathrm{~m} / \mathrm{z}$

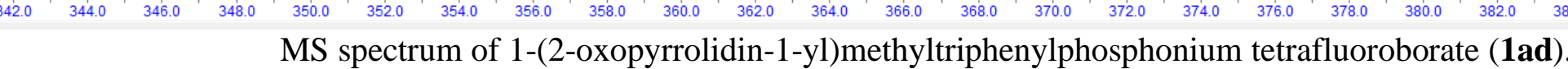




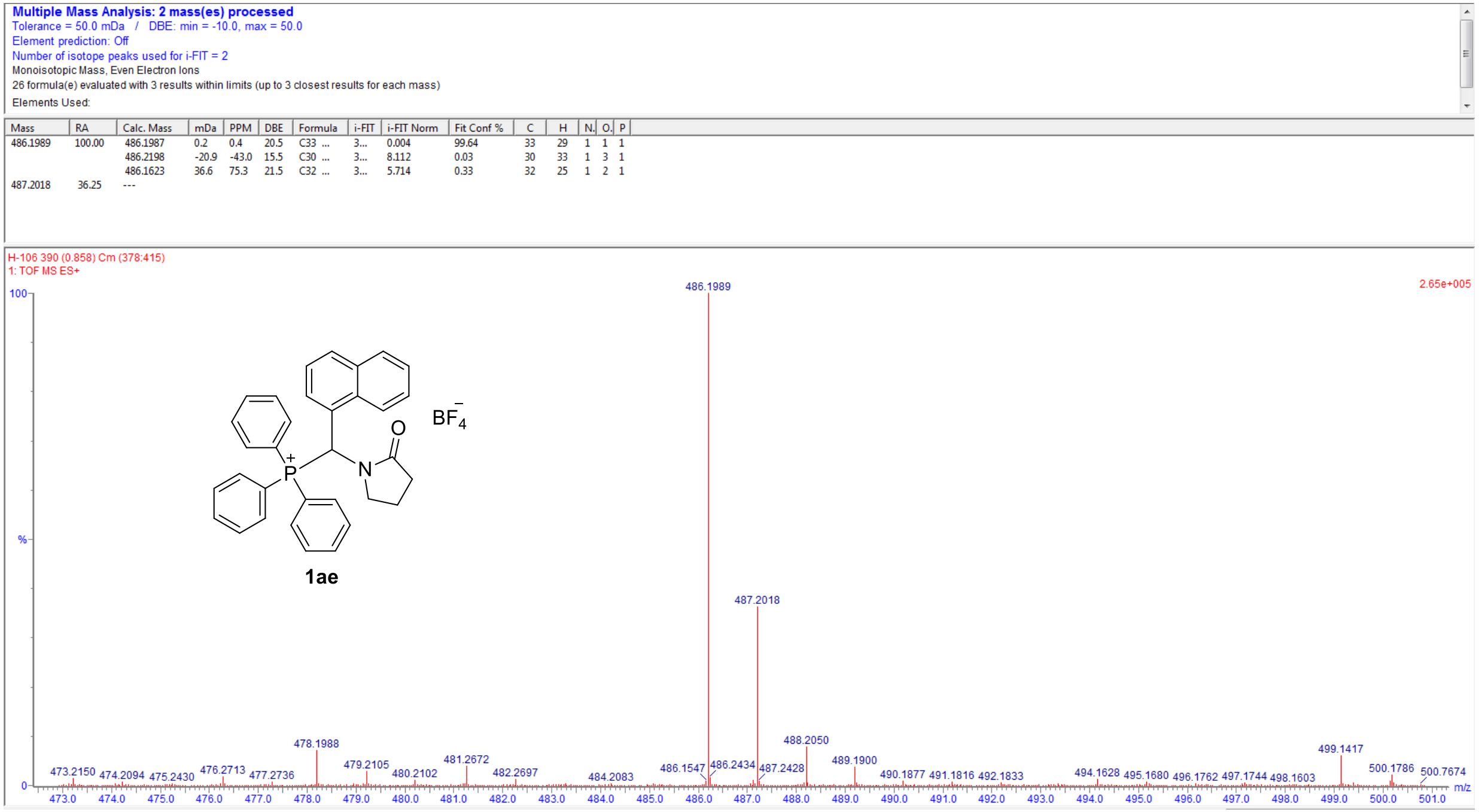

MS spectrum of 1-(2-oxopyrrolidin-1-yl)-1-(1-naphthyl)methyltriphenylphosphonium tetrafluoroborate (1ae). 


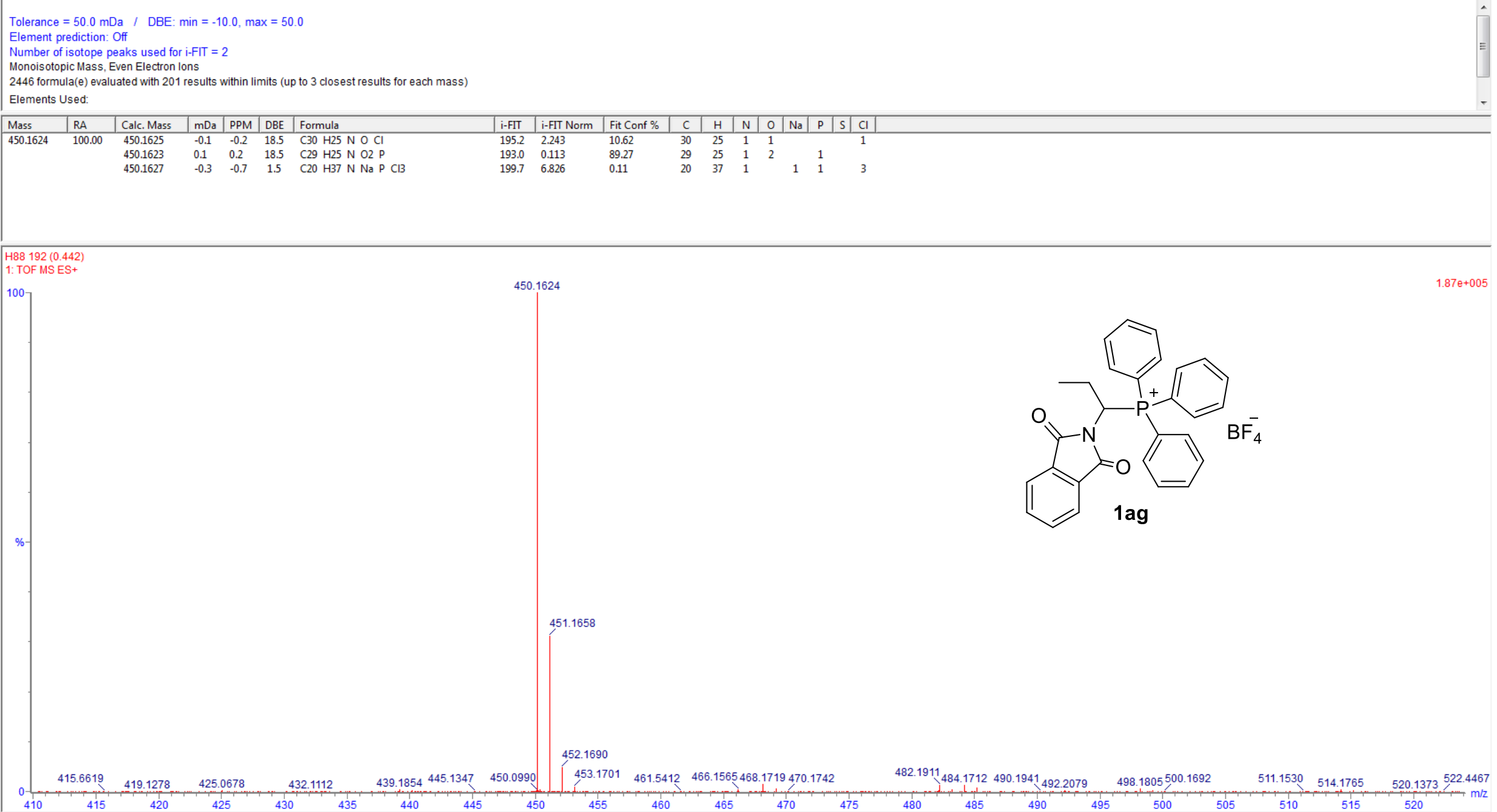

MS spectrum of 1-( $N$-phthalimido)propyltriphenylphosphonium tetrafluoroborate (1ag). 
Number of isotope peaks used for $\mathrm{i}-\mathrm{FIT}=2$

Monoisotopic Mass, Even Electron lons

2

Elements Used:

\begin{tabular}{|c|c|c|c|c|c|c|c|c|c|c|c|c|c|c|}
\hline Mass & RA & Calc. Mass & $\mathrm{mDa}$ & PPM & DBE & Formula & i-FTT & i-FIT Nor & Fit Conf \% & c & H & $\mathrm{N}$ & \begin{tabular}{l|l}
0 & $\mathrm{Na}$
\end{tabular} & 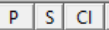 \\
\hline 402.1624 & 100.00 & 402.1623 & 0.1 & 0.2 & $\begin{array}{l}14.5 \\
145\end{array}$ & $\mathrm{C} 25 \mathrm{H} 25 \mathrm{~N} 02 \mathrm{P}$ & 310.8 & 0.273 & 76.11 & 25 & 25 & 1 & & \\
\hline & & $\begin{array}{l}402.2625 \\
4021627\end{array}$ & $\begin{array}{l}-0.1 \\
-0.3\end{array}$ & $\begin{array}{l}-0.2 \\
-0.7\end{array}$ & $\begin{array}{l}14.5 \\
-2.5\end{array}$ & $\mathrm{C} 16 \mathrm{H} 37 \mathrm{~N}$ Na $\mathrm{P} C \mathrm{Cl}$ & 315.0 & $\begin{array}{l}1.482 \\
4.436\end{array}-3$ & $\begin{array}{l}2.11 \\
1.18\end{array}$ & $\begin{array}{l}26 \\
16\end{array}$ & 37 & $\begin{array}{l}1 \\
1\end{array}$ & 1 & 1 \\
\hline
\end{tabular}

${ }^{100}$

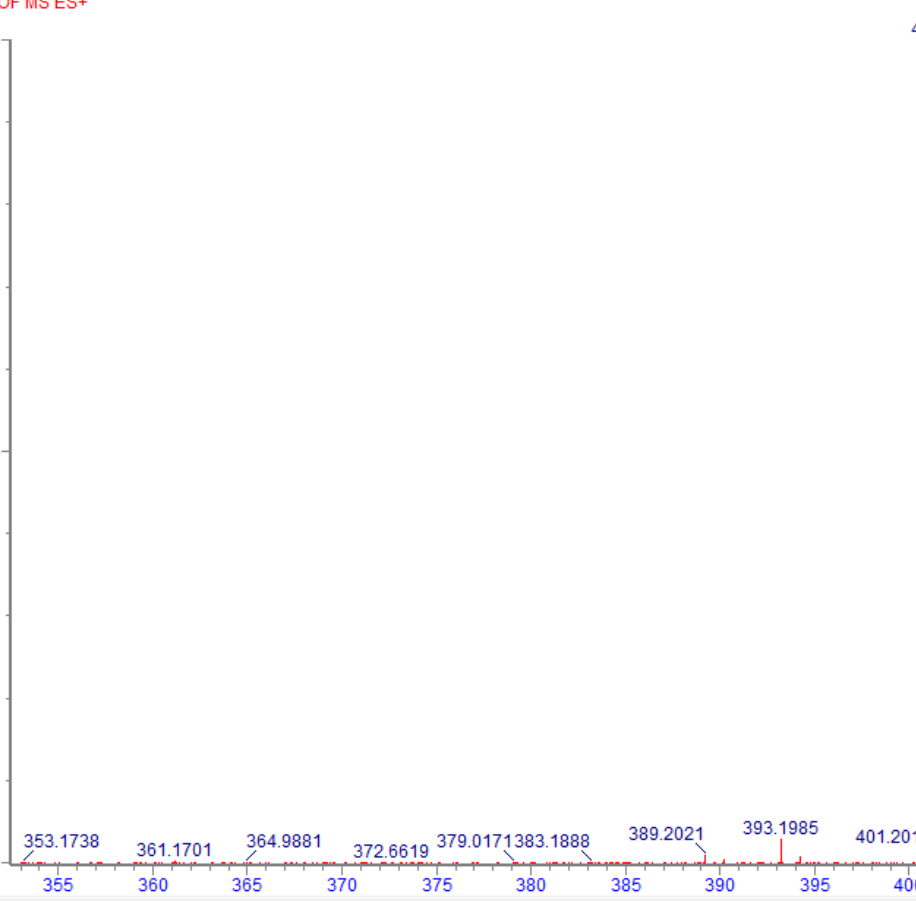

$5.94 \mathrm{e}+005$

MS spectrum of 1-( $N$-succinimido)propyltriphenylphosphonium tetrafluoroborate (1aj). 
Tolerance $=50.0 \mathrm{mDa} / \mathrm{DBE}: \min =-10.0, \max =50.0$

Element prediction: Off

Number of isotope peaks used for $\mathrm{i}-\mathrm{FIT}=2$

2432 tormula(e) evaluated with 195 results within limits (up to 3 closest results for each mass)

Elements Used:

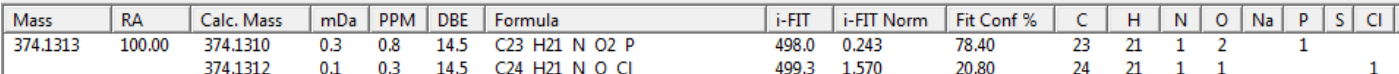

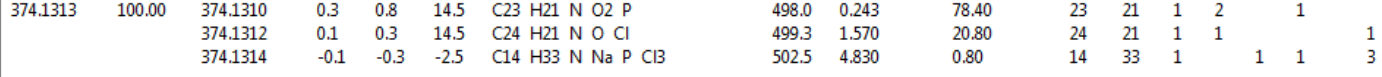

PZ37 260 (0.591) Cm (259:286)
1: TOF MS ES+

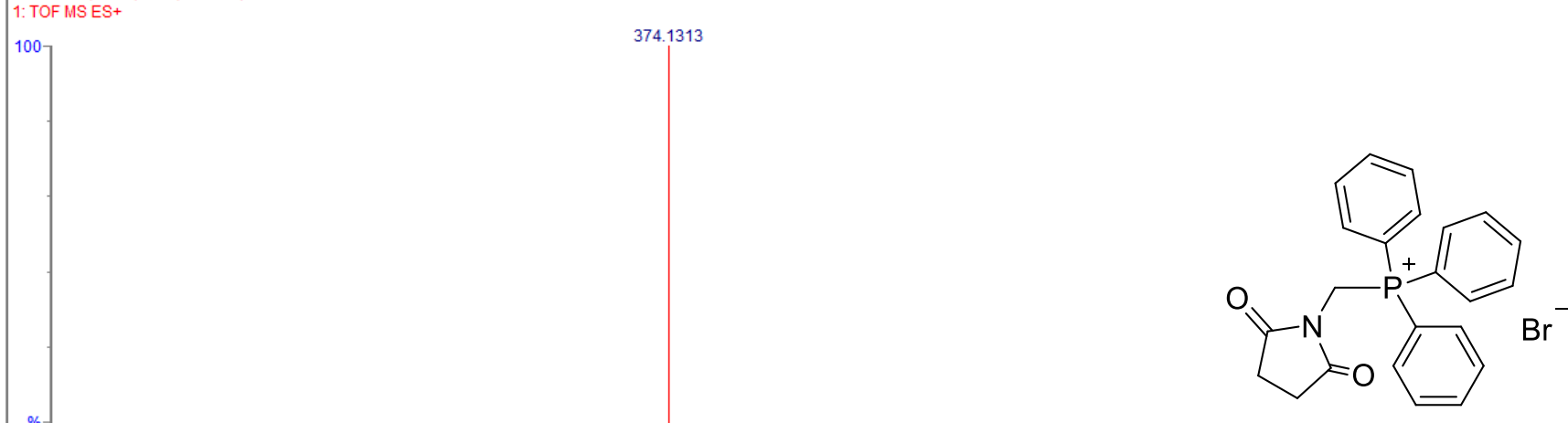

$\mathrm{Br}$

1 ak

75.1346

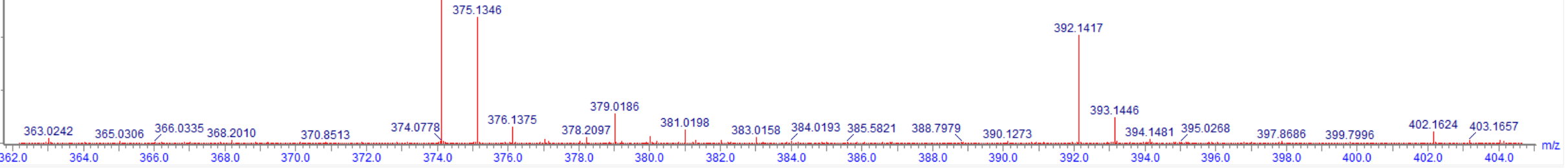

MS spectrum of 1-( $N$-succinimido)methyltriphenylphosphonium bromide (1ak). 
Number of isotope peaks used for I-FIT $=2$

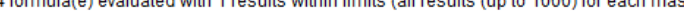

\begin{tabular}{l|l|l|l|l|l|l|l|l|l|l|l|l|}
\hline Mass & RA & Calc. Mass & mDa & PPM & DBE & Formula & i-FIT & i-FT Norm & Fit Conf \% & C & H & P \\
\hline 263.0995 & 100.00 & 263.0990 & 0.5 & 1.9 & 11.5 & C18 H16 P & 323.7 & n/a & n/a & 18 & 16 & 1
\end{tabular}

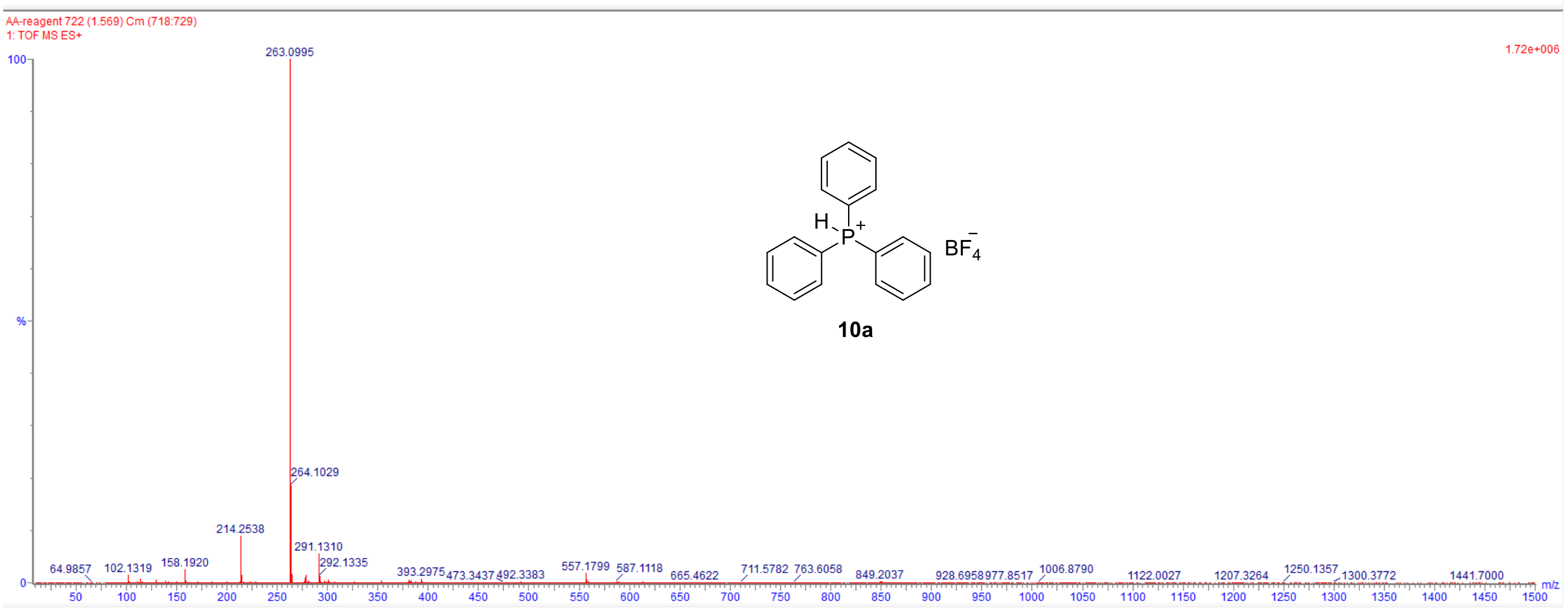

MS spectrum of triphenylphosphonium tetrafluoroborate (10a). 


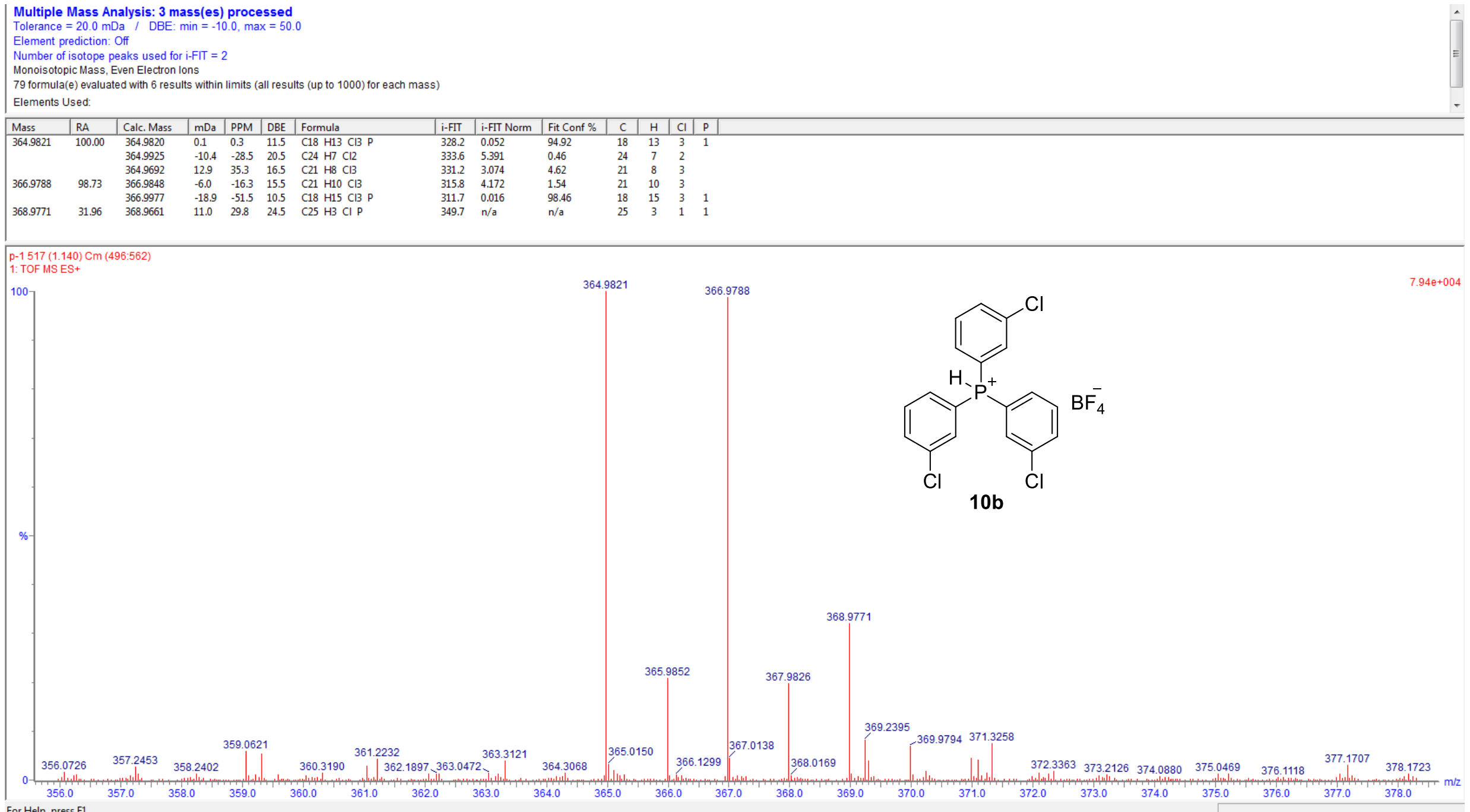

For Help, press F1

MS spectrum of tris(3-chlorophenyl)phosphonium tetrafluoroborate (10b). 
Tolerance $=100.0 \mathrm{mDa} /$ DBE: $\min =-10.0, \max =50.0$

Element prediction: Off

Number of isotope peaks used for $\mathrm{i}-\mathrm{FIT}=2$

17 formula(e) evaluated with 7 results within limits (up to 5 closest results for each mass)

Elements Used:

\begin{tabular}{|c|c|c|c|c|c|c|c|c|c|c|c|c|}
\hline \multirow{6}{*}{$\begin{array}{l}\text { Mass } \\
353.1307\end{array}$} & RA & Calc. Mass & $\mathrm{mDa}$ & PPM & DBE & Formula & i-FIT & i-FIT Norm & Fit Conf $\%$ & C & $\mathrm{H}$ & 0 \\
\hline & 100.00 & 353.1307 & 0.0 & 0.0 & 11.5 & $\mathrm{C} 21 \mathrm{H} 2203 \mathrm{P}$ & 460.7 & 0.058 & 94.36 & 21 & 22 & 1 \\
\hline & & 353.1095 & 21.2 & 60.0 & 16.5 & C24 H18 O P & 465.7 & 5.076 & 0.62 & & & 1 \\
\hline & & 353.1670 & $-36.3 \mathrm{r}>\mathrm{C}$ & -102.8 & 10.5 & $\mathrm{C} 22 \mathrm{H} 26 \mathrm{O} 2 \mathrm{P}$ & 463.8 & 3.168 & 4.21 & 22 & 26 & 2 \\
\hline & & 353.0731 & 57.6 & 163.1 & 17.5 & $\mathrm{C} 23 \mathrm{H} 1402 \mathrm{P}$ & 465.9 & 5.279 & 0.51 & 23 & 14 & 2 \\
\hline & & 353.2034 & -72.7 & -205.9 & 9.5 & $\mathrm{C} 23 \mathrm{H} 30 \mathrm{OP}$ & 466.4 & 5.822 & 0.30 & 23 & 30 & 1 \\
\hline
\end{tabular}

H0 490 (1.068) Cm (454:490)
1: TOF MS ES+

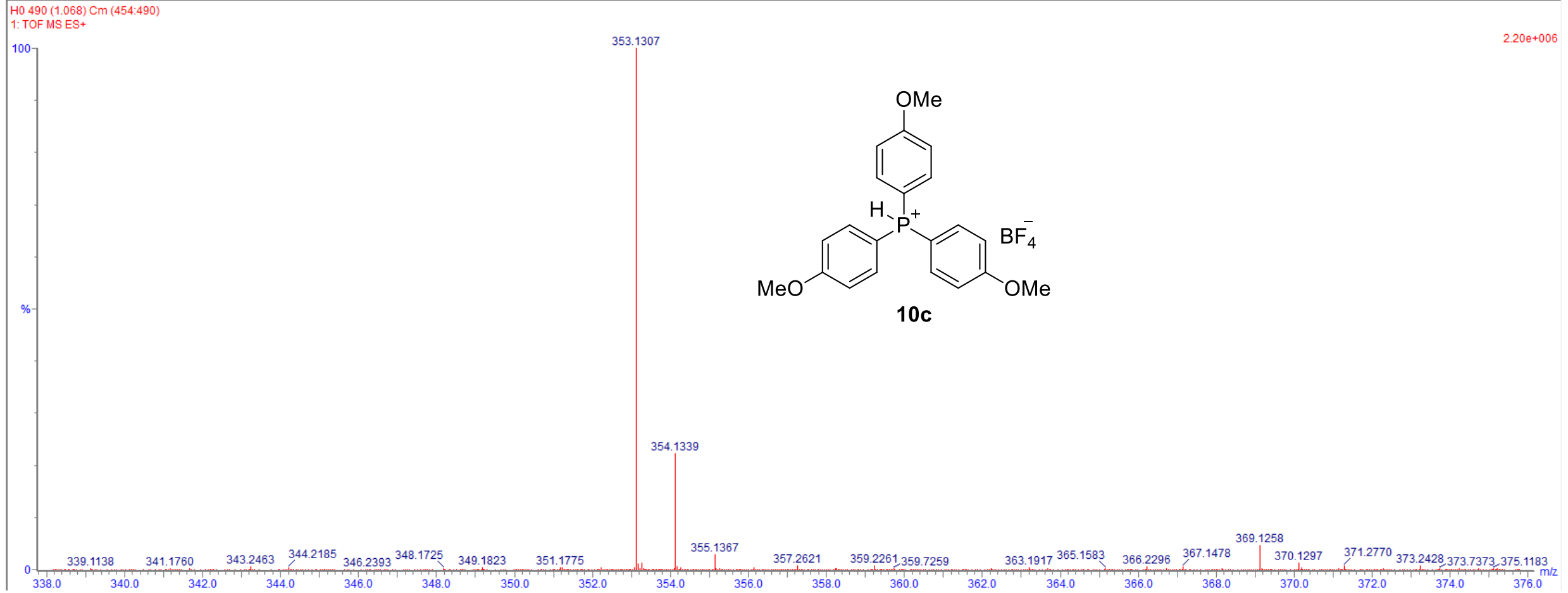

MS spectrum of tris(4-methoxyphenyl)phosphonium tetrafluoroborate (10c). 
Tolerance $=100.0 \mathrm{mDa} /$ DBE: $\min =-10.0, \max =50.0$

Element prediction: Off

Number of isotope peaks used for $\mathrm{i}-\mathrm{FIT}=$

15 formula(e) evaluated with 7 results within limits (up to 5 closest results for each mass)

Elements Used:

\begin{tabular}{|l|l|l|l|l|l|l|}
\hline Mass & RA & Calc. Mass & mDa & PPM & DBE & Formula \\
\hline 321.1408 & 100.00 & 3211408 & 00 & 00 & 115 & C1 \\
\hline
\end{tabular}

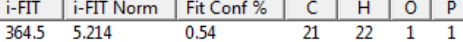

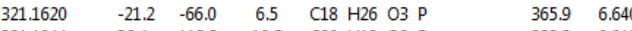

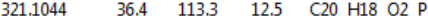

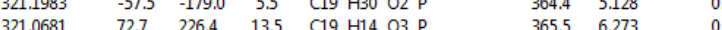

$359.3 \quad 0.015$

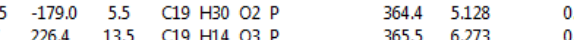

$\begin{array}{llll}18 & 22 & 1 & 1 \\ 12 & 26 & 3 & 1 \\ 19 & 13 & 2 & 1\end{array}$

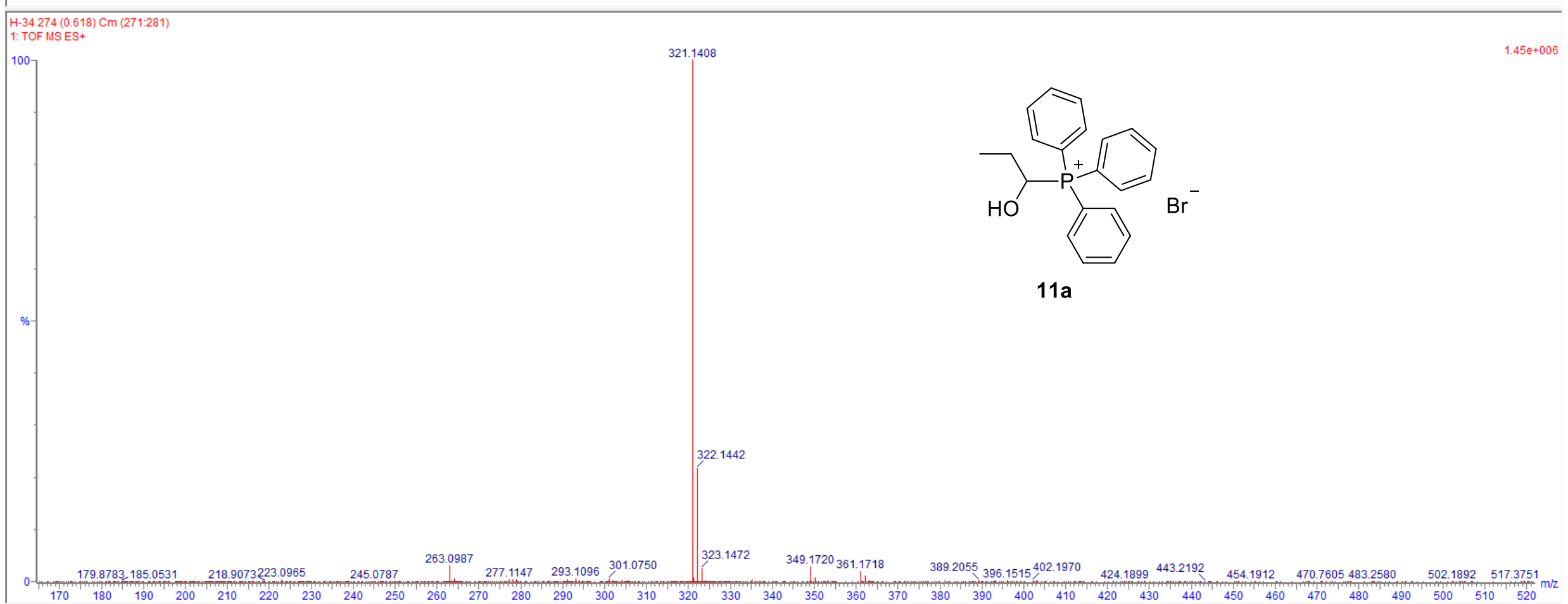

MS spectrum of 1-hydroxypropyltriphenylphosphonium bromide (11a). 


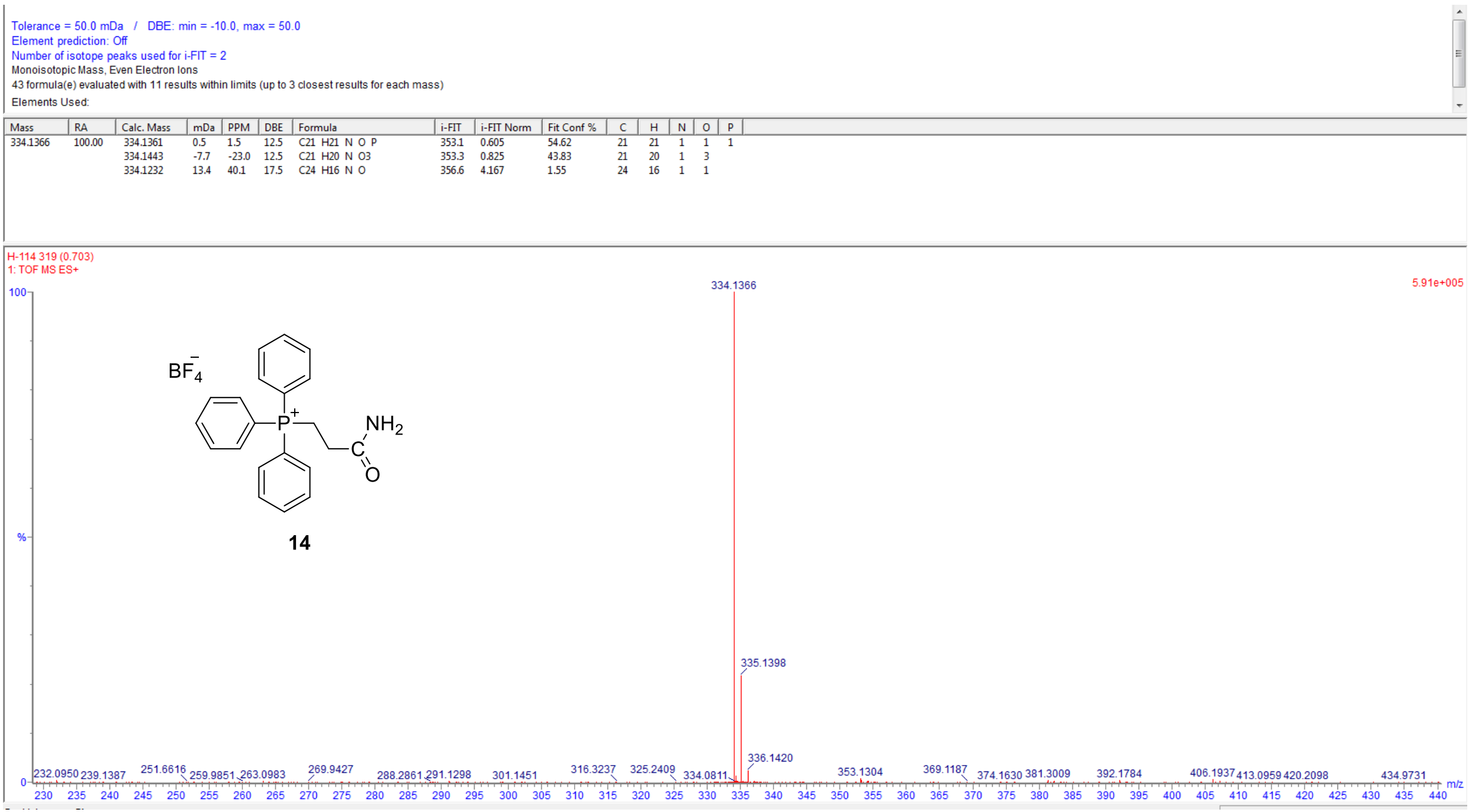

MS spectrum of 2-carbamoyletyhyltriphenylphosphonium tetrafluoroborate (14). 


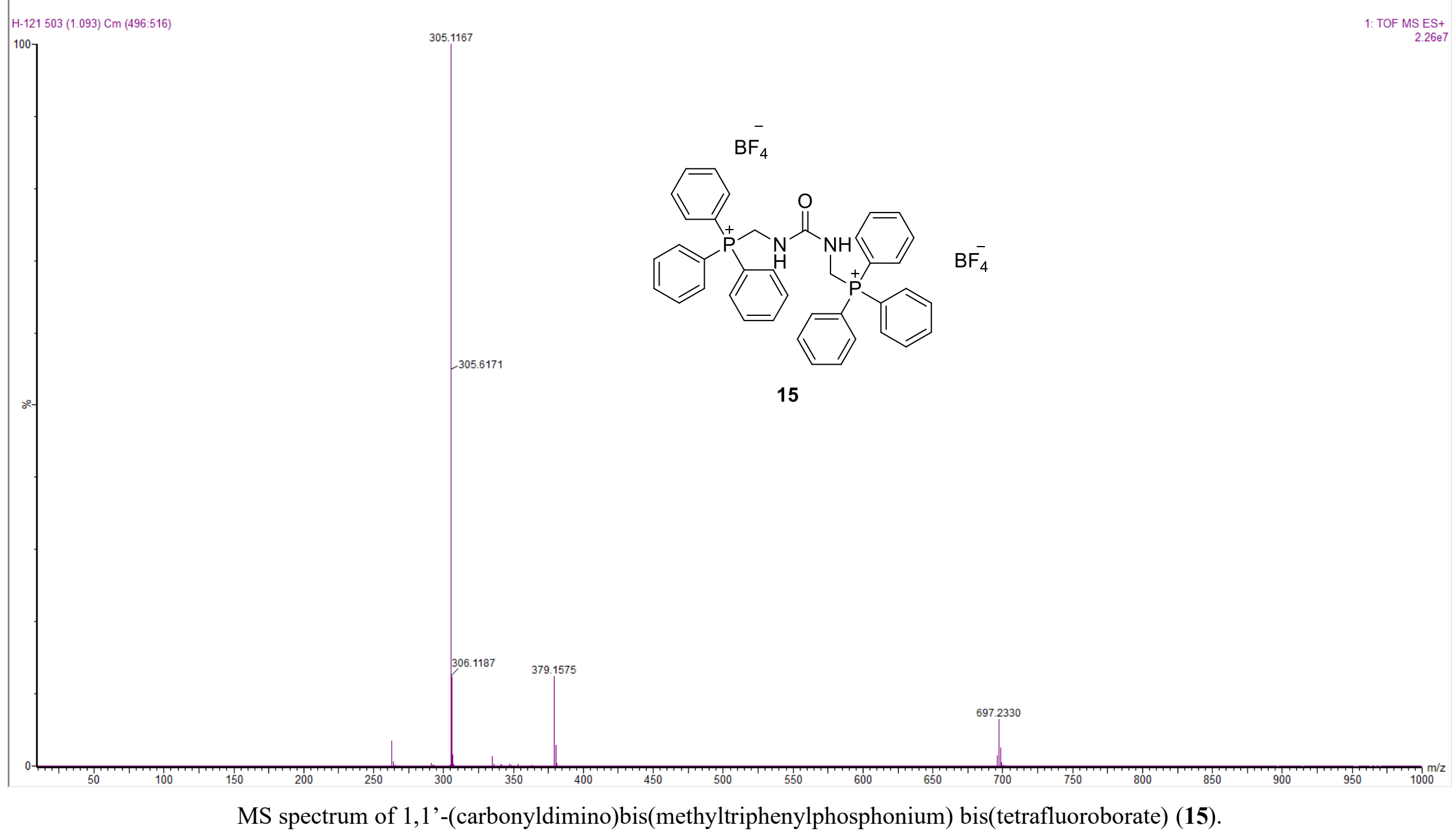

